\title{
of the American Mathematical Society
}

March 2018

Volume 65, Number 3

In Honor of Women's History Month

page 248

AMS Spring Central Sectional Sampler

page 307

Graduate Student Section

page 317
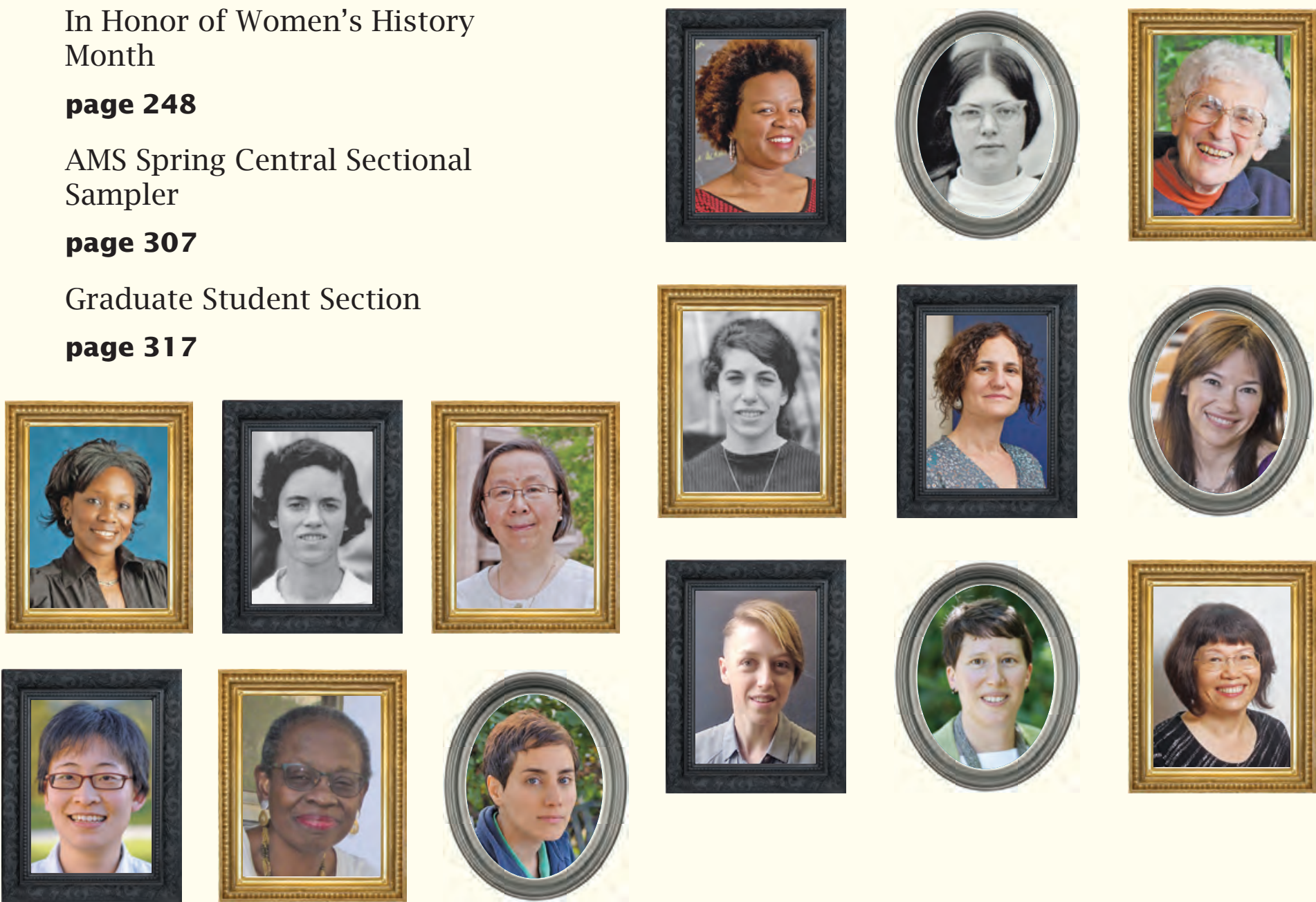

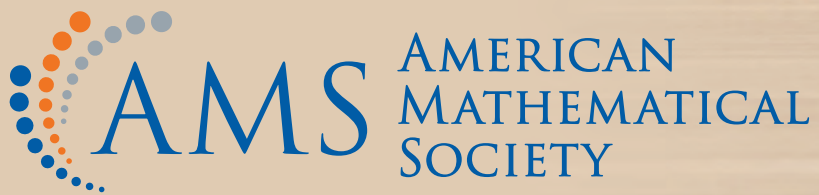
About the Issue, with cover key, on page 242. 


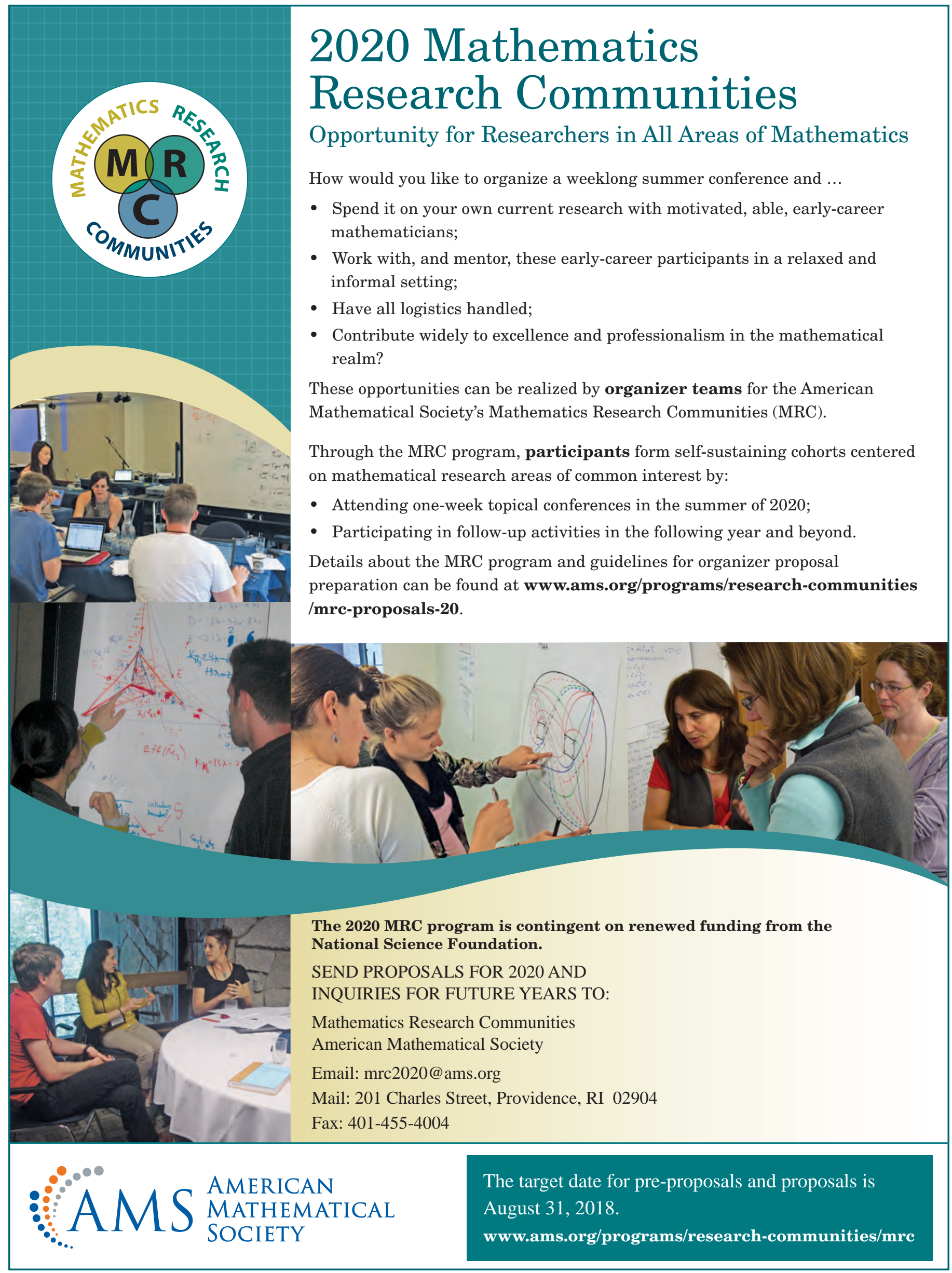




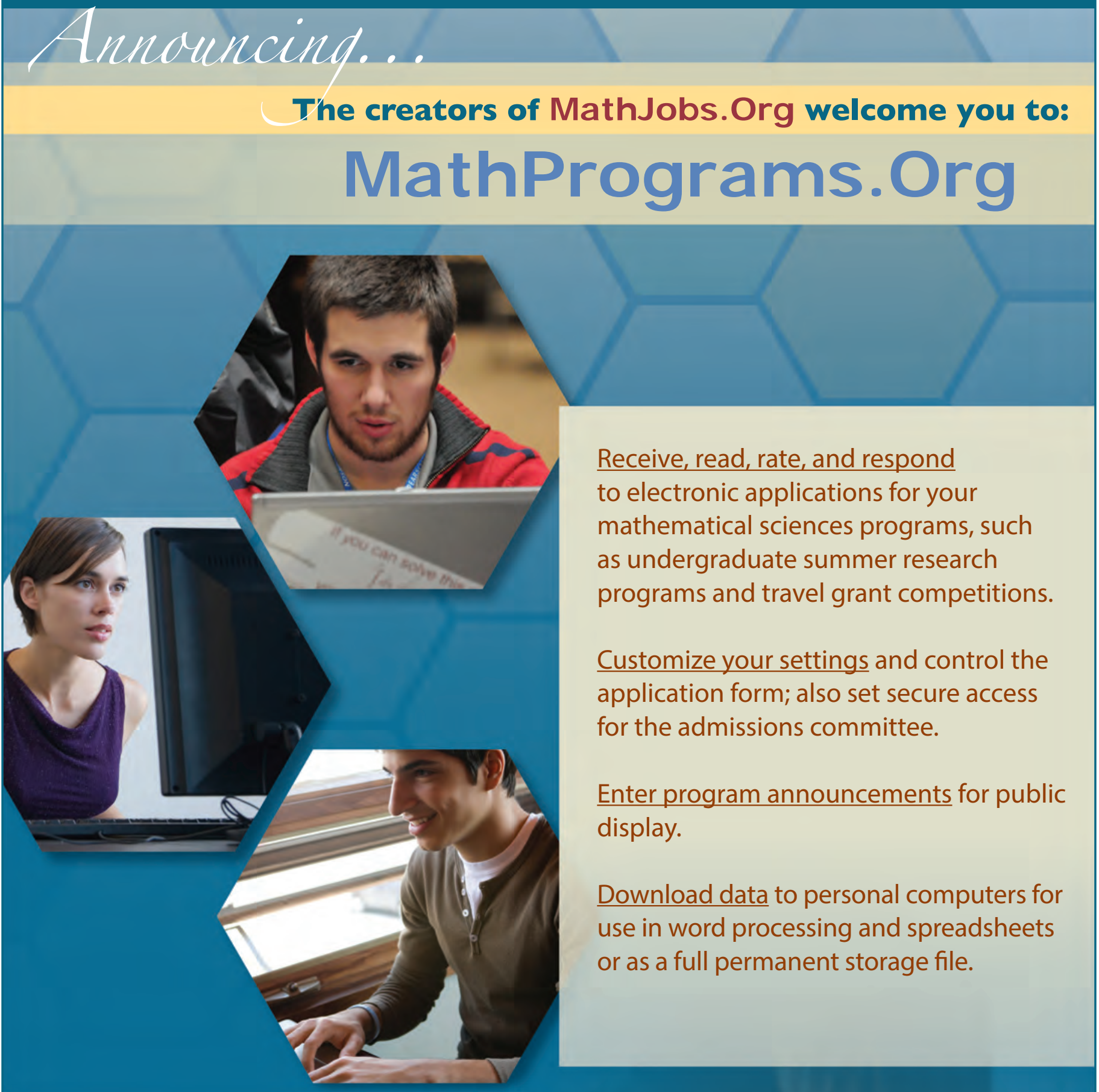

Service is FREE to applicants.

Institutions pay annually for one program or for multiple programs.

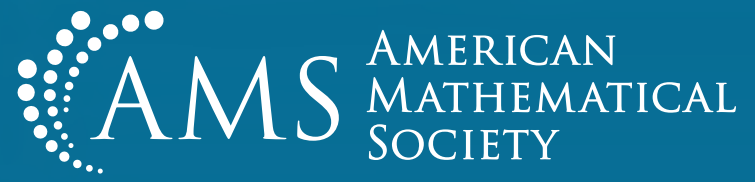


In honor of Women's History Month, guest editors Margaret A. Readdy and Christine Taylor have assembled profiles of 27 mathematicians and more. These profiles provide inspiring and humbling reminders of how some individuals have contributed to mathematics, often under very difficult circumstances, and how much we need to welcome and respect all.
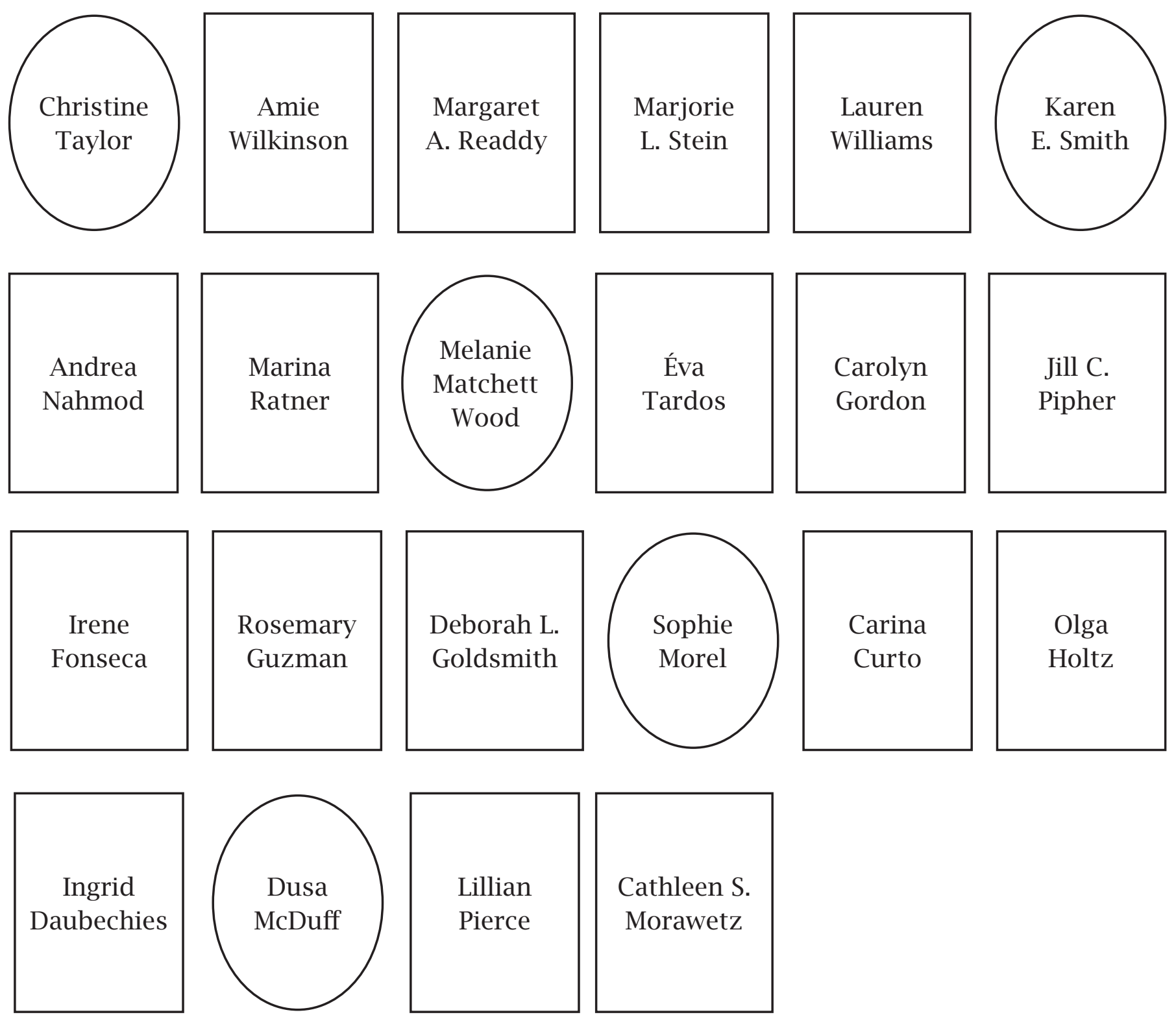

Cathleen S.

Morawetz 

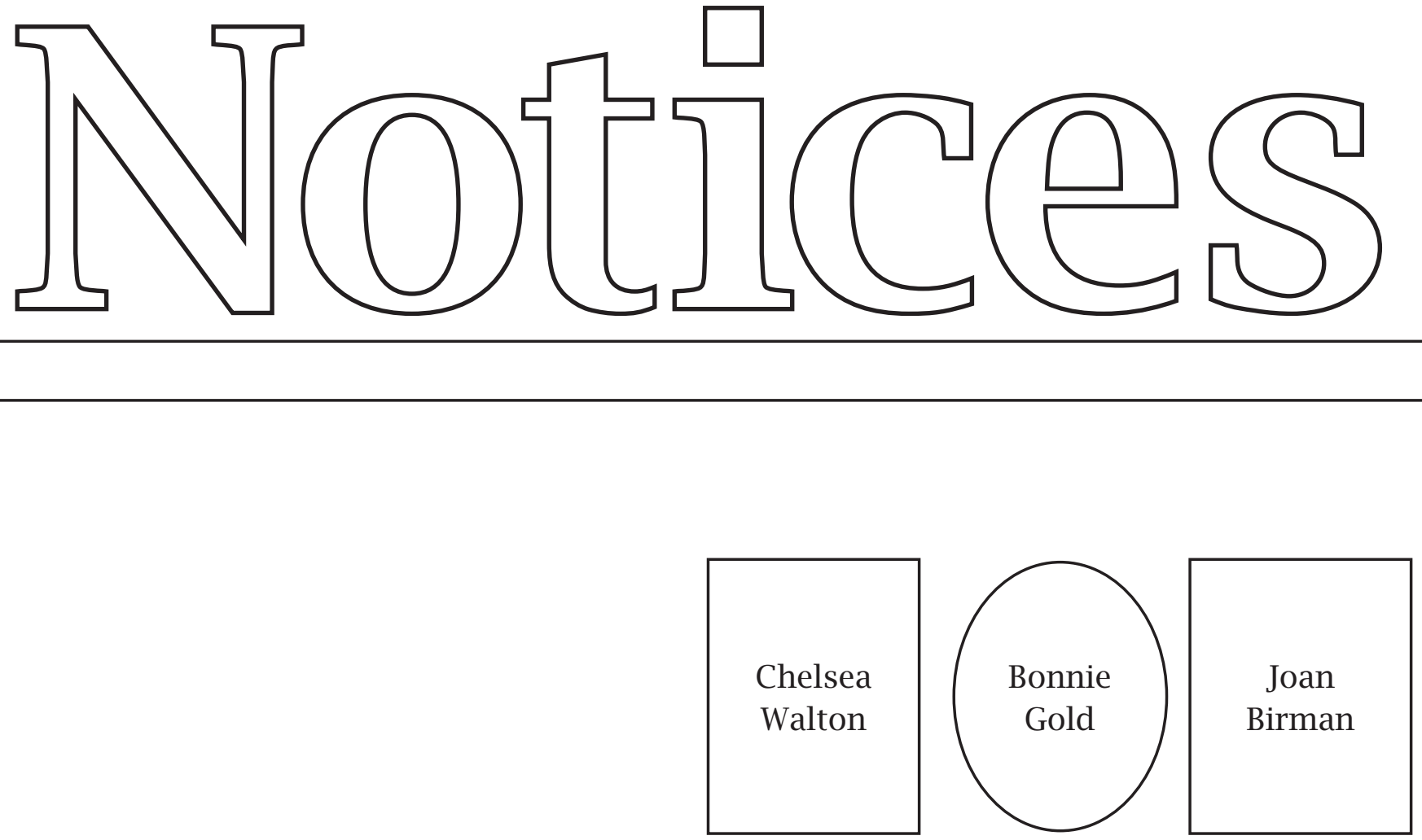
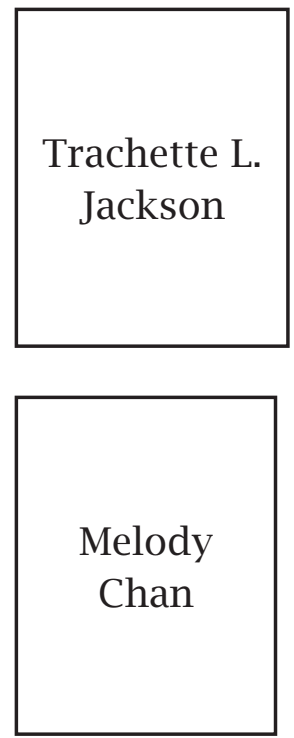
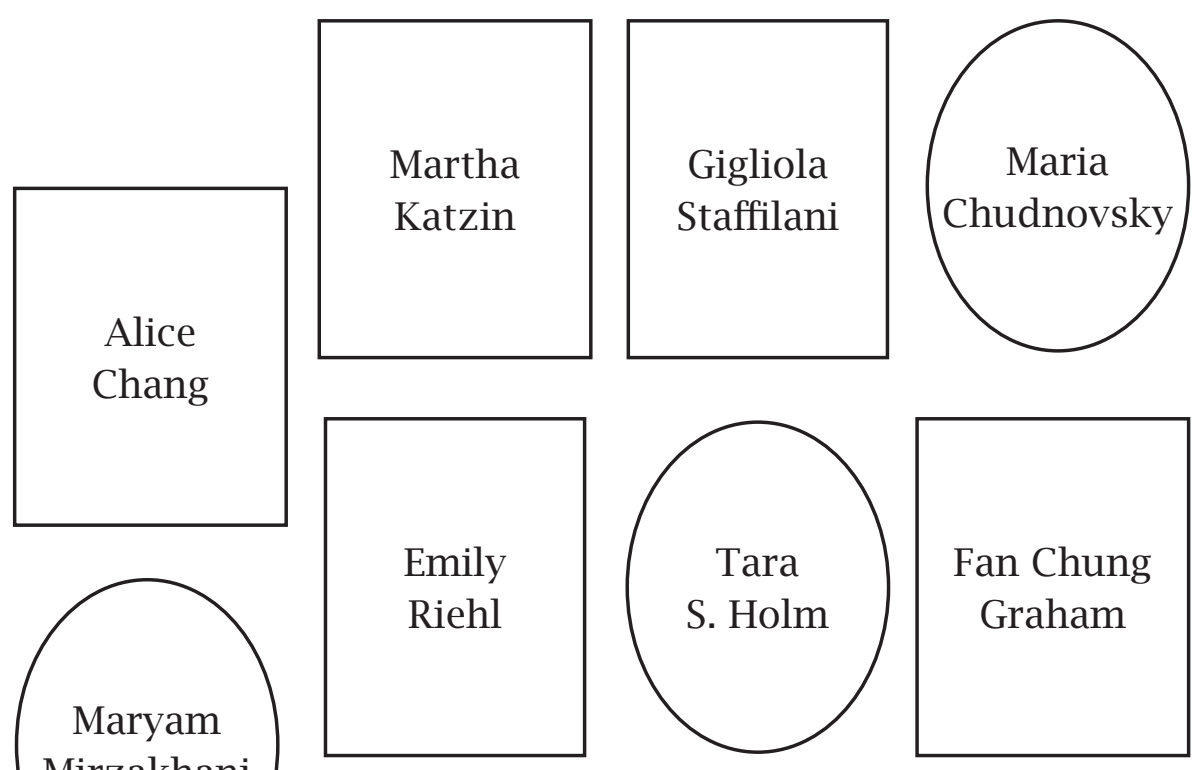

Fan Chung

Graham 

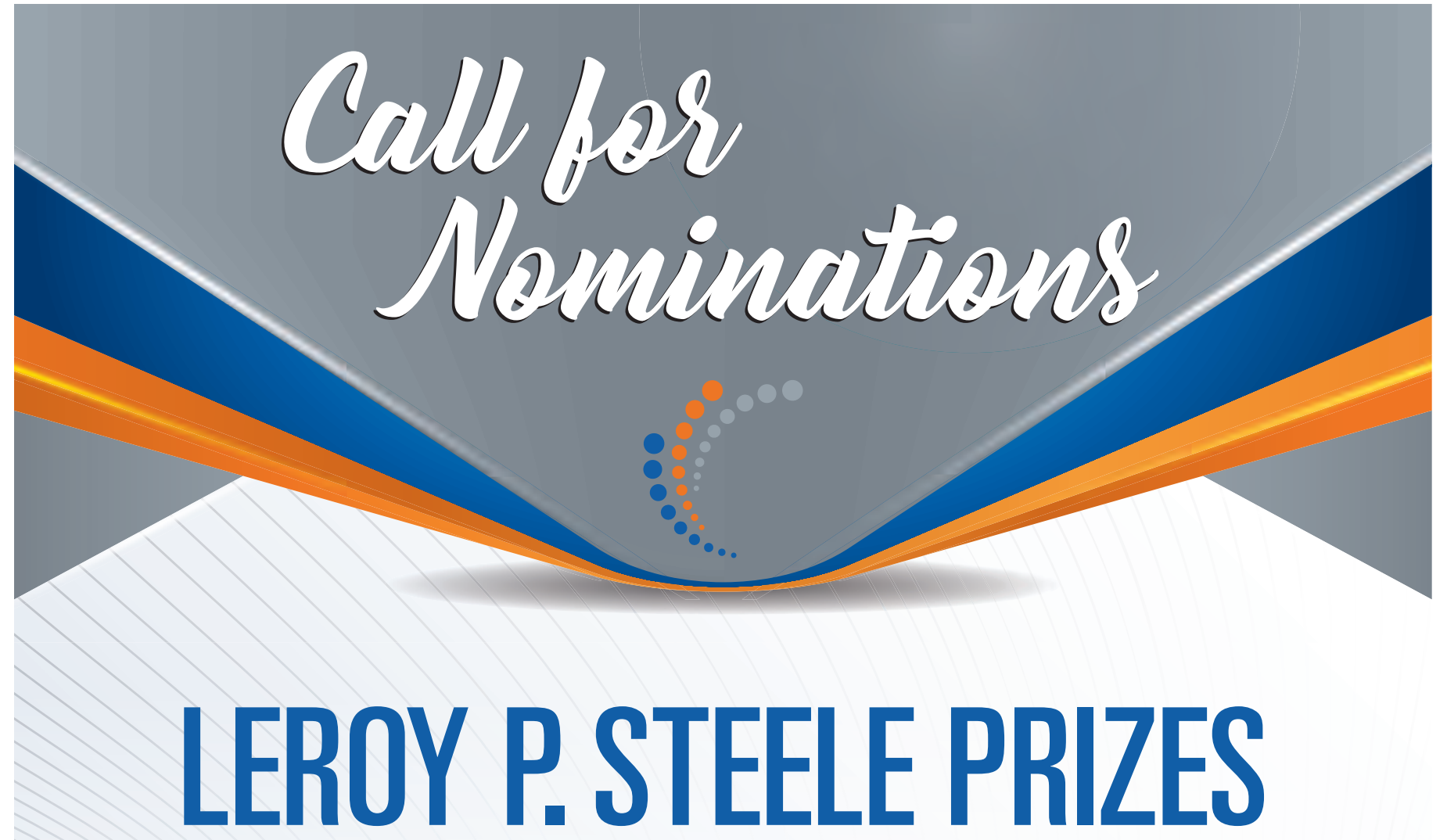

The selection committee for these prizes requests nominations for consideration for the 2019 awards. Further information about the prizes can be found in the November 2017 Notices p.1203 (also available at www.ams.org/steele-prize).

Three Leroy P. Steele Prizes are awarded each year in the following categories: (1) the Steele Prize for Lifetime Achievement: for the cumulative influence of the total mathematical work of the recipient, high level of research over a period of time, particular influence on the development of a field, and influence on mathematics through PhD students; (2) the Steele Prize for Mathematical Exposition: for a book or substantial survey or expository-research paper; and (3) the Steele Prize for Seminal Contribution to Research: for a paper, whether recent or not, that has proved to be of fundamental or lasting importance in its field, or a model of important research. In 2019 the prize for Seminal Contribution to Research will be awarded for a paper in mathematics (open subject area).

Further information and instructions for submitting a nomination can be found at the Leroy $\mathrm{P}$. Steele Prizes website: www.ams.org/steele-prize.

Nominations for the Steele Prizes for Lifetime Achievement and for Mathematical Exposition will remain active and receive consideration for three consecutive years.

For questions contact the AMS Secretary at secretary@ams.org.

The nomination period is February 1, 2018 through March 31, 2018.

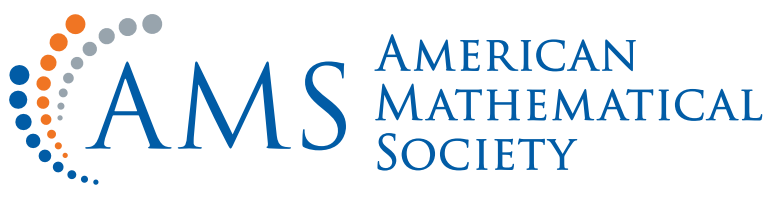




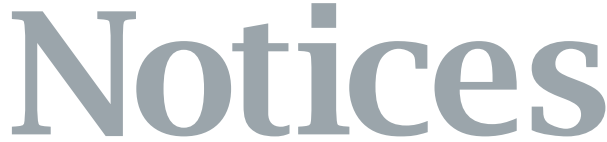

of the American Mathematical Society
Women...should be fearless

to sepk those who are willing to inuest in and heln grow, their talent.

\section{FEATURED}

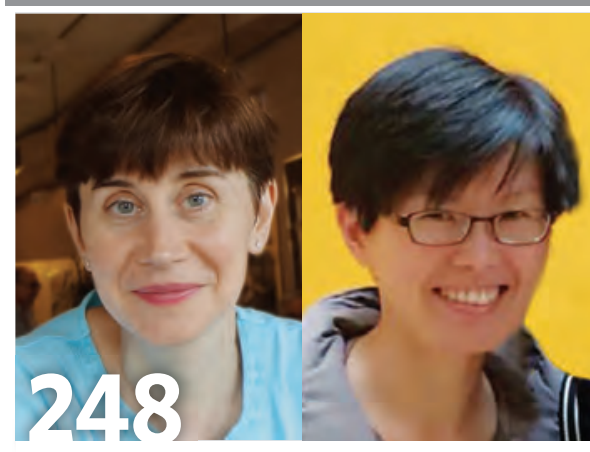

\section{Women's History Month}

Introduction

Profiles of Twenty-Seven

Mathematicians

Early Women Mathematicians in Princeton

Guest Editors Margaret A. Readdy and Christine Taylor

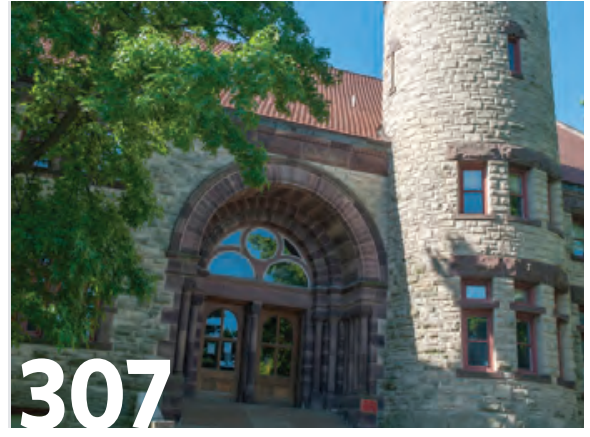

\section{Spring Central Sectional} Sampler

Recent Progress in the Zimmer Program

by Aaron Brown

Bi-Lipschitz Equivalence of Groups by Tullia Dymarz

The Correlation Constant of a Field by June Huh

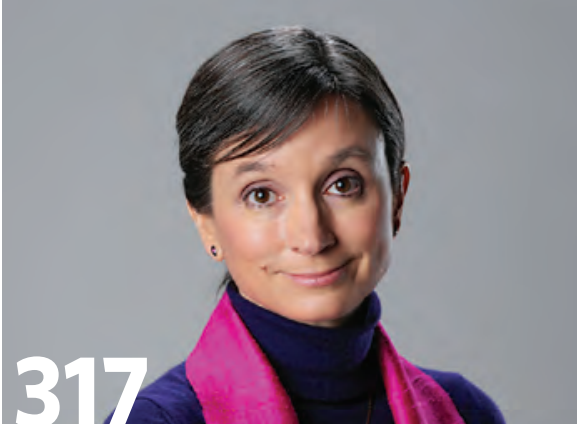

\section{Graduate Student Section}

Interview with Kathryn Hess Conducted by Melinda Lanius

WHAT IS...an Information Projection? by Frank Nielsen

Our special section for Women's History Month provides inspiring and humbling reminders of how much some individuals have contributed to mathematics, often under very difficult circumstances, and how much we need to welcome and respect all. First in our minds are three recently deceased mathematical giants-Maryam Mirzakhani, Cathleen Morawetz, and Marina Ratner-with memorials planned for future issues. Marie A. Vitulli provides two reports: one on "Gender Differences in First Jobs..." and another on Wikipedia pages about women mathematicians. We are happy to report that for Notices the number of women authors rose from 35 (16\%) in 2015 to $61(30 \%)$ in 2017. We all need to combat discrimination and prejudice within ourselves and others. UCI Math's Alice Silverberg has an eye-opening collection of experiences and observations on the subject in her blog Adventures in Numberland (see: https://si tes. google.com/site/numberlandadventures). This issue also includes a lecture sampler for the March AMS Spring Central Sectional at Ohio State University, an interview with Kathryn Hess, and “WHAT IS...an Information Projection?" by Frank Nielsen.

Enjoy the month and the math. -Frank Morgan, Editor-in-Chief

\section{ALSO IN THIS ISSUE}

313 Open Problems Concerning Michell Trusses Wilfrid Gangbo

326 Gender Differences in First Jobs for New US PhDs in the Mathematical Sciences

Marie A. Vitulli

330 Writing Women in Mathematics into Wikipedia Marie A. Vitulli

335 Letters to the Editor

341 Herbert Busemann Athanase Papadopoulos

350 Report on 2015-2016 New Doctoral Recipients Amanda L. Goldbeck, Thomas H. Barr, and Colleen A. Rose

\section{ALSO IN THIS ISSUE, CONT'D.}

360 Report on 2015-2016 Employment Experiences of the New Doctoral Recipients Amanda L. Goldbeck, Thomas H. Barr, and Colleen A. Rose

\section{FROM THE AMS SECRETARY.}

244. Call for Nominations: 2019 Leroy P. Steele Prizes

3462018 AMS Class of Fellows

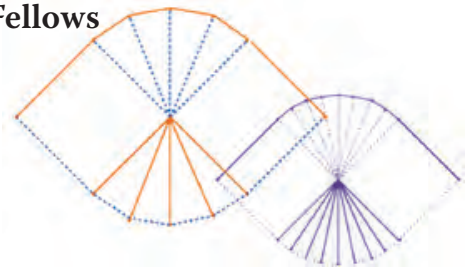




\section{IN EVERY ISSUE}

337 Mathematics People

34.4 Mathematics Opportunities

366 New Publications Offered by the AMS

368 Classified Advertising

371 Meetings and Conferences of the AMS

384 In the Next Issue

\section{CURRENT INVITATIONS FROM THE AMS}

cover 2 MRC 2020

241 MathPrograms.Org

244. Call for Nominations: 2019 Leroy P. Steele Prizes

3122018 AMS Einstein Public Lecture in Mathematics

325 Start an AMS Graduate Student Chapter

345 Join the AMS

349 Annual Survey: Data of Interest to You

370 Join MathSciNet ${ }^{\circledR}$

380 Call for Proposals for JMM 2019

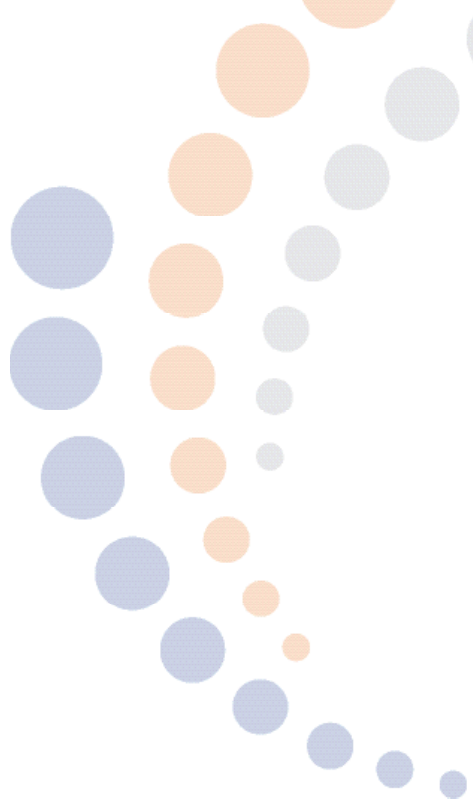

\section{INVITATION TO \\ NUMBER THEORY}

Oystein Ore

\section{SECOND EDITION}

Revised and updated

by John J. Watkins and Robin Wilson

With the addition

of new material

on Fermat's Last

Theorem, the role

of computers in number theory, and the use of number theory in cryptography, this introduction to number theory is in line with contemporary developments in the field. The authors have also made numerous minor changes in the presentation and layout of the text and the exercises.

Anneli Lax New Mathematical Library, Volume 49; 2017; 134 pages; Softcover; ISBN: 978-0-88385-653-6; List US\$48; AMS members

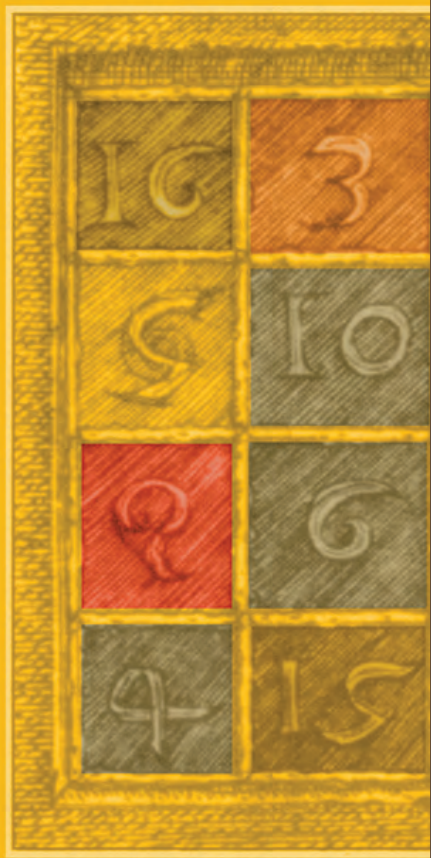
US\$38.40; Order code NML/49

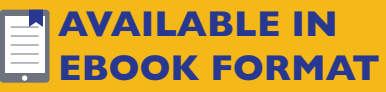

ORDER YOUR COPY AT BOOKSTORE.AMS.ORG/NML-49

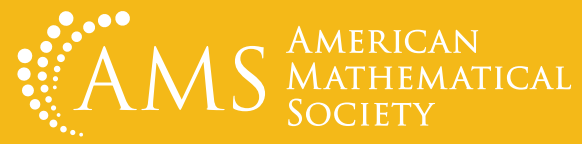




\section{EDITOR-IN-CHIEF \\ Frank Morgan}

\section{ASSOCIATE EDITORS}

Benjamin Braun,

Alexander Diaz-Lopez, Thomas Garrity, Joel Hass,

Stephen Kennedy, Florian Luca, Steven J. Miller, Harriet Pollatsek, Carla Savage (ex officio),

Cesar E. Silva, Christina Sormani, Daniel J. Velleman

CONSULTANTS

John Baez, Hélène Barcelo, Ricardo Cortez, Jerry Folland, Tara Holm, Kenneth A. Ribet

\section{ASSISTANT to the EDITOR-IN-CHIEF Sophia D. Merow}

MANAGING EDITOR Rachel L. Rossi

ADVERTISING COORDINATOR

Anne Newcomb

REPRINT PERMISSIONS Erin M. Buck

CONTRIBUTING WRITER

Elaine Kehoe

COMPOSITION, DESIGN, and EDITING

Brian Bartling, John Brady, Anna Hattoy, Lori Nero,

Karen Ouellette, Rebecca Rivard, Courtney Rose,

Deborah Smith, Mike Southern, Peter Sykes

Supported by the AMS membership, most of this publication, including the opportunity to post comments, is freely available electronically through the AMS website, the Society's resource for delivering electronic products and services. Use the URL www.ams.org/notices/to access the Notices on the website. The online version of the Notices is the version of record, so it may occasionally differ slightly from the print version.

The print version is a privilege of Membership. Graduate students at member institutions can opt to receive the print magazine by updating their individual member profiles at https://www.ams. org/cml/update-ams. For questions regarding updating your profile, please call 800-321-4267.

For back issues see www.ams.org/backvols. Note: Single issues of the Notices are not available after one calendar year.

\section{CONTACTING THE NOTICES}

\section{SUBSCRIPTION INFORMATION}

Subscription prices for Volume 65 (2018) are US\$662 list; US\$529.60 institutional member; US\$397.20 individual member; US $\$ 595.80$ corporate member. (The subscription price for members is included in the annual dues.) A late charge of $10 \%$ of the subscription price will be imposed upon orders received from non-members after January 1 of the subscription year. Add for postage: Surface delivery outside the United States and IndiaUS\$27; in India-US\$40; expedited delivery to destinations in North America-US\$35; elsewhere-US\$120. Subscriptions and orders for AMS publications should be addressed to the American Mathematical Society, PO Box 845904, Boston, MA 02284-5904 USA. All orders must be prepaid.

\section{ADVERTISING}

Notices publishes situations wanted and classified advertising, and display advertising for publishers and academic or scientific organizations. Advertising requests, materials, and/or questions should be sent to:

\section{classads@ams.org (classified ads) notices-ads@ams.org (display ads)}

\section{PERMISSIONS}

All requests to reprint Notices articles should be sent to: reprint-permission@ams.org

\section{SUBMISSIONS}

The editor-in-chief should be contacted about articles for consideration after potential authors have reviewed the "For Authors" page at www.ams.org/publications/journals/notices/ noticesauthors.

The managing editor should be contacted for additions to our news sections and for any questions or corrections. Contact the managing editor at: notices@ams.org

\section{Letters to the editor should be sent to: notices-letters@ams.org}

Additions to the Math Calendar should be submitted at: www.ams.org/cgi-bin/mathcal/mathcal-submit.pl

To make suggestions for additions to other sections, and for full contact information, see www.ams.org/publications/ journals/notices/noticescontact

[Notices of the American Mathematical Society (ISSN 0002-9920) is published monthly except bimonthly in June/July by the American Mathematical Society at 201 Charles Street, Providence, RI 02904-2213 USA, GST No. 121892046 RT ${ }^{\star \star \star \star *}$. Periodicals postage paid at Providence, RI, and additional mailing offices. POSTMASTER: Send address change notices to Notices of the American Mathematical Society, P.O. Box 6248, Providence, RI 02904-6248 USA.] Publication here of the Society's street address and the other bracketed information is a technical requirement of the US Postal Service.

(C) Copyright 2018 by the American Mathematical Society. All rights reserved.

Printed in the United States of America. The paper used in this journal is acid-free and falls within the guidelines established to ensure permanence and durability. 


\section{Women's History Month}

\section{Margaret A. Readdy and Christine Taylor, Guest Editors}

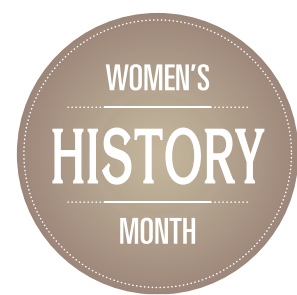

The history of women in mathematics in the US started more than 130 years ago. Winifred Edgerton Merrill ${ }^{1}$ became the first woman to receive $\mathrm{a} \mathrm{PhD}$ (1886) in mathematics in the US at Columbia. Christine Ladd had earned the degree from Johns Hopkins four years earlier, but it was not granted until 1926. The University of Chicago was a leading producer of PhDs for women in mathematics prior to World War II. These early doctorate recipients include Anna Pell Wheeler (1910), the first woman to deliver an invited address to the AMS (1923) and later chairwoman of Bryn Mawr's mathematics department; Pauline Sperry (1916), who spent most of her career at Berkeley; and Mayme Irwin Logsdon (1921), the only woman to hold a regular faculty position above the rank of instructor at Chicago until Karen Uhlenbeck became professor there in 1982. Prominent women mathematicians in the same generation also include Olga Taussky-Todd ${ }^{2}$ (1930, Vienna); Mina Rees $^{3}$ (1931, Chicago); Dorothy Bernstein (1939, Brown), the first woman president of the MAA; and Julia Robinson ${ }^{4}$ (1948, Berkeley), the first woman president of the AMS.

Margaret A. Readdy is professor of mathematics at the University of Kentucky. Her email address is margaret. readdy@uky. edu.

Christine Taylor is senior lecturer of mathematics at Princeton University. Her email address is ctay 1or@math . princeton . edu.

Readdy and Taylor are, respectively, current and former program managers of the Women and Mathematics Program at the Institute for Advanced Study. Readdy and Taylor wish to thank Jeanne LaDuke for advice, expertise, and help, both historical and editorial.

${ }^{1}$ See the article "Winifred Edgerton Merrill: 'She Opened the Door'” in the April 2012 Notices www.ams.org/notices/201204/ rtx120400504p.pdf

2See the article "In Memoriam: Olga Taussky-Todd" in the August 1996 Notices www . ams . org/notices/199608/taussky . pdf.

${ }^{3}$ See the article "Mina Spiegel Rees (1902-1997)" in the August 1998 Notices www . ams . org/notices/199807/memoria1-rees. pdf.

For permission to reprint this article, please contact: reprint-permission@ams.org.

DOI: http://dx.doi.org/10.1090/noti1653

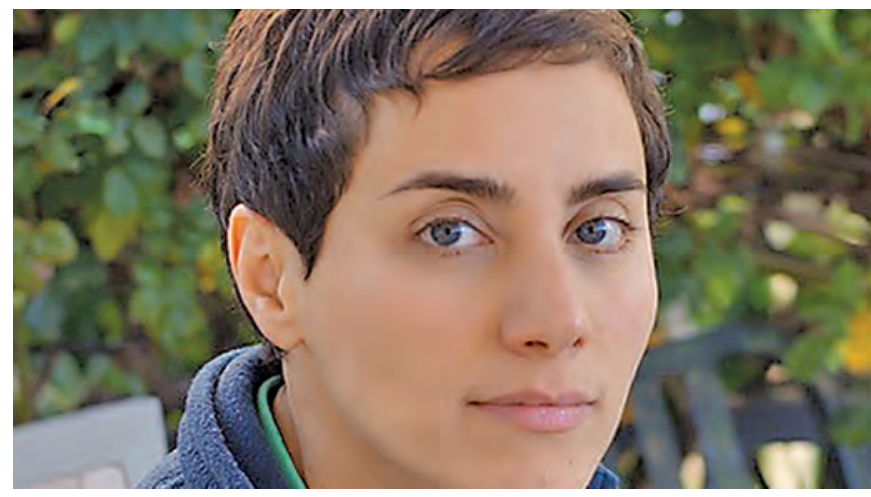

Maryam Mirzakhani (1977-2017)

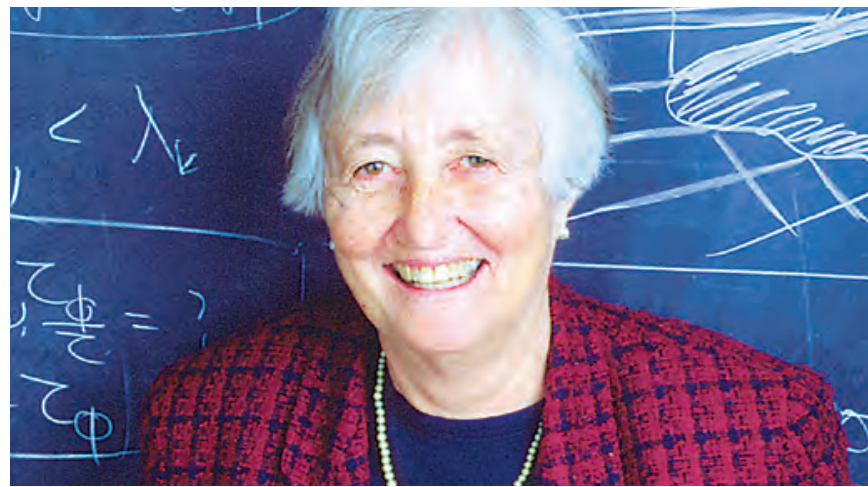

Cathleen Synge Morawetz (1923-2017)

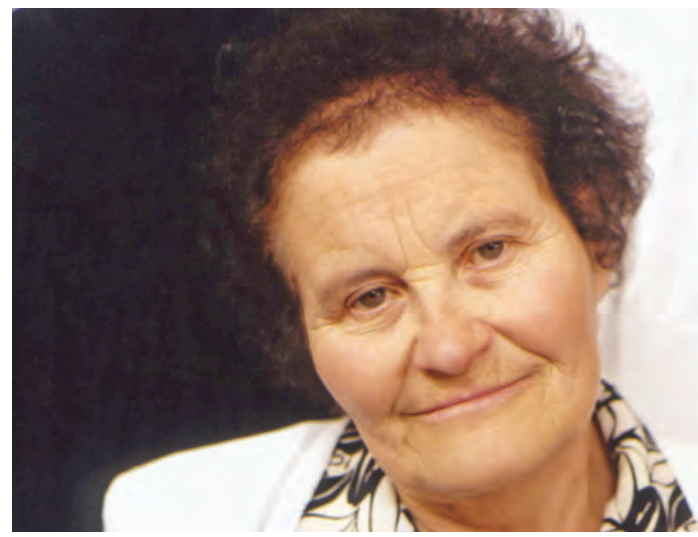

Marina Ratner (1938-2017) 
Generally, the women who earned doctorates in mathematics before WWII did their work with distinguished advisors, but had difficulties when it came to finding jobs, especially at institutions comparable to those where they had received their education. Women's colleges and coeducational private colleges were often more supportive and nurturing.

After WWII, the percentage of women earning PhDs in mathematics dropped dramatically and only returned to its earlier levels in the 1980s. Contemporary women mathematicians come from a variety of backgrounds, as exemplified by the accompanying profiles and this $\mathrm{No}$ tices cover. In recent years, women mathematicians have entered the American workforce from every continent. Within the US, women from diverse social, economic, and racial backgrounds have slowly found their footing and risen to the forefront in mathematics. We take this opportunity to honor some of them.

While much progress has been made in the last thirty years in training and retaining women in mathematics through governmental and institutional policies, the woefully low percentage of women with prestigious awards and recognitions highlights the task ahead for the mathematical community.

\section{In Memoriam:}

In the summer of 2017, the mathematical community mourned the passing of three mathematical giants:

Maryam Mirzakhani, ${ }^{5}$ who won the Fields Medal in 2014, worked in Teichmüller theory, hyperbolic geometry, ergodic theory, and symplectic geometry;

Cathleen Synge Morawetz, ${ }^{6}$ who received the National Medal of Science in mathematics in 1998, the AMS Leroy P. Steele Prize for Lifetime Achievement in 2004, and was AMS President 1995-1996, worked in partial differential equations; and

Marina Ratner, best known for Ratner's theorems on unipotent flows on homogeneous spaces, which she proved in her 50s, worked in ergodic theory.

\section{ABOUT THE EDITORS}

Margaret A. Readdy works in algebraic combinatorics. Readdy spent a sabbatical at MIT in 2006-2007, was a member of the Institute for Advanced Study in 2010-2011, and spent a sabbatical and research leave at Princeton University in 2014-2015.

Christine Taylor studied manifolds with exceptional holonomy in graduate school and more recently has worked on evolutionary game theory.

\section{Photo Credits}

Photo of Maryam Mirzakhani courtesy of Jan Vondrák, 2012. Photo of Cathleen Morawetz by James Hamilton.

Photo of Marina Ratner authored by George M. Bergman. Source: Archives of the Mathematisches Forschungsinstitut Oberwolfach.

Photo of Margaret A. Readdy courtesy of Theodore Ehrenborg. Photo of Christine Taylor courtesy of Christine Taylor.

EDITOR'S NOTE. Memorials for Mirzakhani, Morawetz, and Ratner are planned for future issues.

\footnotetext{
${ }^{4}$ See the article "Julia Robinson and the Hilbert Tenth Problem" in the May 2008 Notices www.ams.org/notices/200805/ tx080500573p.pdf.

${ }^{5}$ Stanford Memorial: www . youtube . com/watch?v=IUfB2HadIBw \&feature=youtu.be.

Harvard Memorial: www . youtube . com/watch?v=HUBnzTTQ5jk

${ }^{6}$ Courant Memorial: cims.nyu.edu/conferences/cath1eenmorawetz-celebration/index.htm1.
} 


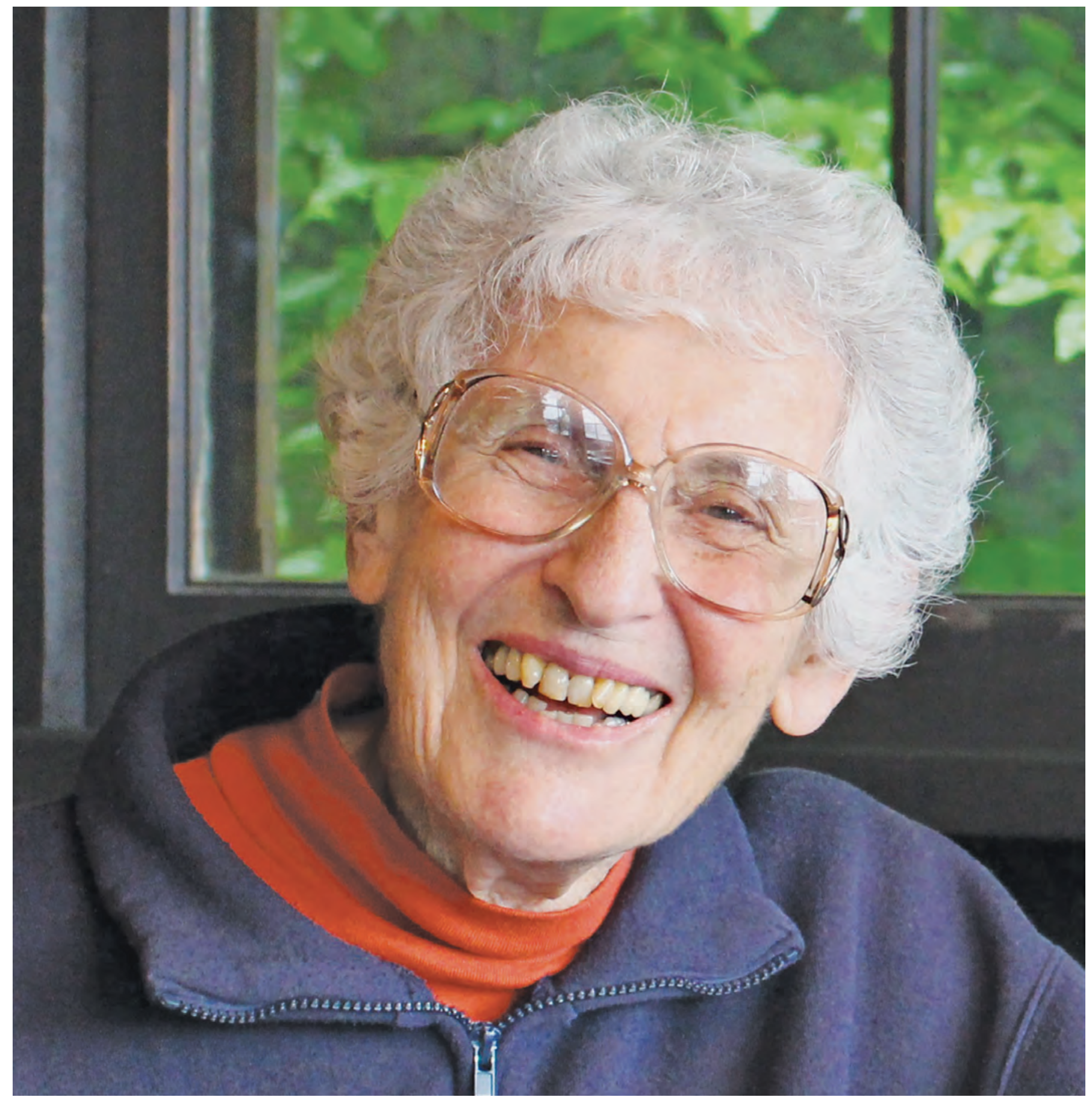


WOMEN'S

HISTORY

MONTH

\section{Synopsis of Research}

In Joan Birman's first formal meeting with her PhD thesis advisor, Wilhelm Magnus, she learned that the mapping class group of the twice-punctured torus was well understood and that she should think about how it might change if there were three punctures. This tiny start eventually led Birman into an area of research that starts with mapping class groups of surfaces, and the combinatorics of curves on surfaces, and moves out to encompass the topology, geometry, and group theory of 3- and 4-manifolds. Braid groups arise in this setting as the mapping class group of the $n$-times punctured disk. Early in Birman's career, she wrote Braids, Links and Mapping Class Groups, which had a wide readership. As it happens, braids are present in widely different parts of mathematics. As one dramatic example, the Jones polynomial, a knot-type invariant, was first discovered via apparently unrelated work in operator algebras. Gradually, it became clear that braiding had made its appearance through the way in which one type $\mathrm{II}_{1}$ factor sat inside another.

\section{Biography}

I was a math major in college, and after graduation I chose to work in industry (at what was then called "systems analysis"), full time for nine years and part time for four years. I began graduate studies with evening courses after my third child was born and received my $\mathrm{PhD}$ from the Courant Institute of NYU in 1968, exactly twenty years after my BA. My husband, Joseph L. Birman, professor of physics at NYU, was highly supportive of every step I took, and the confidence I gained from his support, plus the free tuition I obtained as an NYU spouse, were essential parts of that picture. There were very few women in math in the early 1970s, and that plus my age at graduation certainly caused problems, e.g., I received exactly one job offer (for a position at Stevens Institute of Technology), and it came a few weeks before fall classes began! Nevertheless, with that job in hand my career was on its way.

I think that the combination of teaching and research is an excellent one. There was always at least one student in the classes I taught who asked sharp questions and kept me on my toes. My research continued and attracted interest. Among the seminar talks I gave was one at Professor Ralph Fox's Princeton topology seminar. That talk led to an invitation to visit Princeton. ${ }^{1}$ It was not a traditional postdoc at all, just a one-semester visiting appointment with a two-course teaching load, and the logistics required a three-day/week commute between New Rochelle and Princeton: however, it made a huge difference in my career. The course that I gave that semester became a research monograph, with a list of open problems at the end, each one a problem I encountered as I prepared those lectures. The book had a wide readership and eventually led to a move in 1973-4 from Stevens to a tenured position at Barnard-Columbia. The stimulation of belonging to a fine department, with its seminars and colloquia, highly talented postdocs, and excellent graduate students helped my growth as a mathematician. I never felt that I knew enough mathematics, and that is true to this day.

\section{Advice to Young Women}

I think that the most important thing is to identify work that you love. I really love mathematics. As regards to balancing the needs of family and work, think carefully about what is important to you and what will work for you. I note that this is a major issue for most women mathematicians. I note that another major issue for potential mathematicians, male and female, is to not be intimidated by people who understand material faster than you understand it. To be sure, speed helps, but it is not fundamentally important.

\section{Photo Credit}

Photo of Joan Birman $\subset$ Ken Birman, 2014.

\footnotetext{
$\overline{{ }^{1} \text { See also "Early Women Mathematicians in Princeton" on page } 304 .}$
} 
Melody Chan

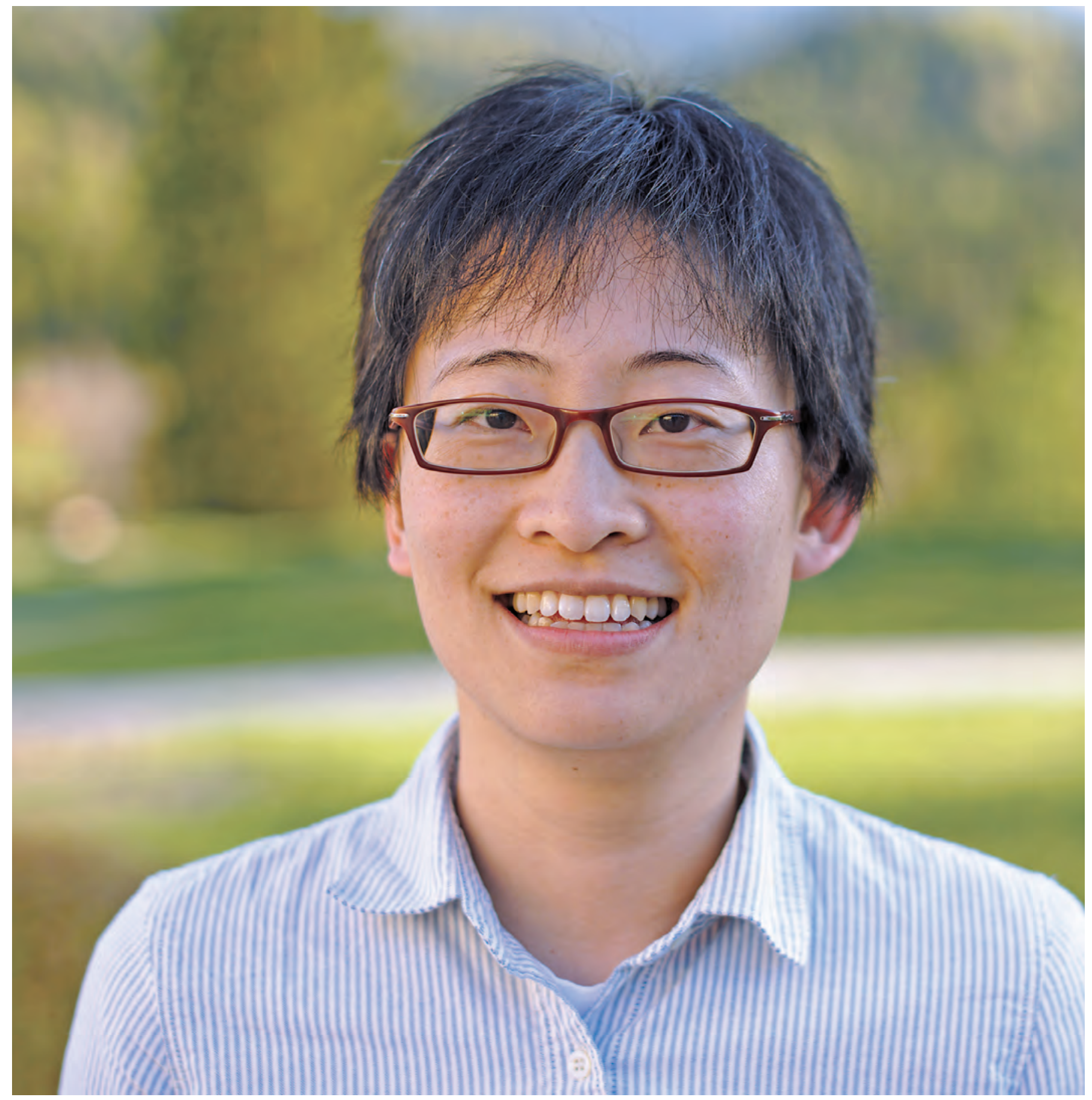


WOMEN'S

\section{HISTORY}

MONTH

\section{Synopsis of Research}

Melody Chan is a mathematician working in the fields of tropical geometry, combinatorial algebraic geometry, and combinatorics. A common thread in her research is using degeneration techniques in algebraic geometry to study curves and their moduli spaces. Tropical geometry is a modern such technique, in which algebraic objects may be regarded as degenerating to entirely combinatorial ones. Chan has authored or co-authored over twenty publications, and has made notable contributions on the topic of tropical curves and their moduli spaces, especially combinatorial, topological, and stack-theoretic foundations.

\section{Biography}

Melody Chan grew up in the suburbs of New York. She juggled a dual love of math and music: after graduating from high school, she went to the Juilliard School at the age of sixteen spending a year studying violin with Itzhak Perlman and Dorothy DeLay. Subsequently she attended Yale University and received her $\mathrm{PhD}$ from the University of California Berkeley in 2012, advised by Bernd Sturmfels. She was an NSF Postdoctoral Fellow at Harvard from 2012 to 2015 before beginning her current position as an assistant professor at Brown University. Chan's research has been supported by recent grants from the National Security Agency, from the National Science Foundation, and by the Henry Merritt Wriston Fellowship, Brown University's highest award for junior faculty for research and teaching. She has also maintained and nurtured a commitment to teaching and mentoring in mathematics since graduate school.

\section{Advice to Young Women}

Do examples! Try to do as much mathematics as you can standing at the board, writing things down, and explaining them to people.

\section{Photo Credit}

Photo of Melody Chan courtesy of Joseph Rabinoff. 


\section{Alice Chang}

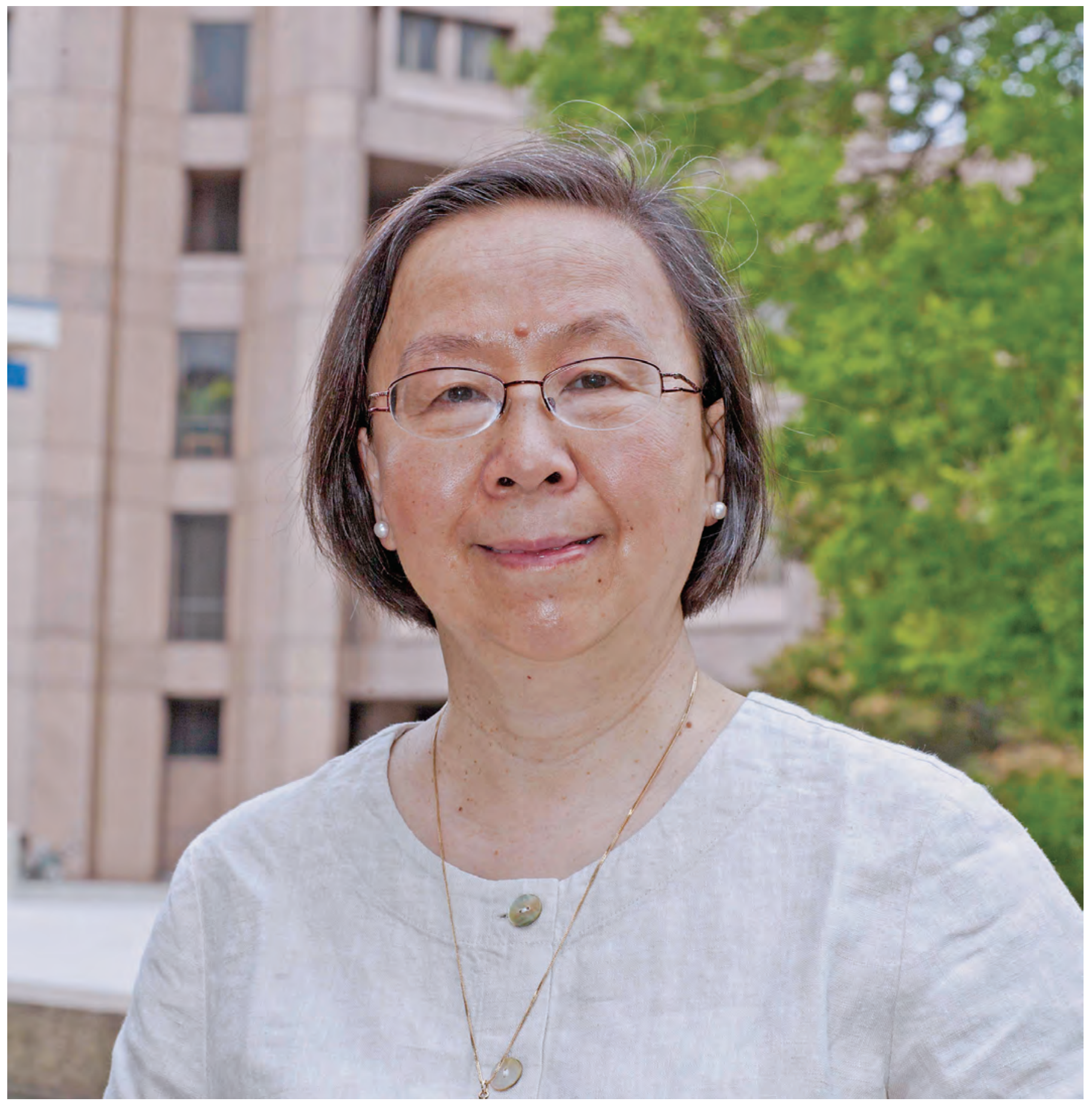




\section{WOMEN'S}

\section{HISTORY}

MONTH

\section{Synopsis of Research}

Alice Chang started her research as a classical harmonic analyst. Her research interests have gradually shifted to problems in geometric analysis, mainly in applying PDE methods to study problems in conformal geometry. Her major contributions include applying PDE techniques to study the Gauss-Bonnet integrand and other conformal invariant quantities on real 4-manifolds. These PDEs are of higher order (in particular 4th order) and fully non-linear (of Monge-Ampère type). One of her goals is to classify the diffeomorphism type of a class of 4-manifolds under some conformally invariant curvature conditions-modestly continuing the great achievement of the geometry in the last decade in classifying the diffeomorphism type of 3-dimensional manifolds.

\section{Biography}

I was born in China, grew up in Taiwan, and have spent most of my life in the US. I think I benefited a lot from my multicultural background. I have lived the life of an academic and a research mathematician and feel I have been really lucky in life. I have a wonderful family: my husband Paul Yang is also a mathematician, a colleague and a co-worker; I have a son and a daughter, both of whom I am proud of. I work in a profession I truly enjoy, I live in a stimulating environment, and I have been treated well in all the places I have worked. One thing I find truly rewarding is supervising $\mathrm{PhD}$ students and watching them grow in the profession; many of them have become close, personal friends of mine. I am profoundly honored in the profession, having been elected to NAS, AAAS, Academia Sinica in Taiwan, and Fellow of the AMS, and having served as chair of the mathematics department at Princeton during 2010-2013.

\section{Advice to Young Women}

In my career I have benefited a lot from collaboration with colleagues. My advice for young women is to pursue your passion with a group of friends with similar interests.

\section{Comments on Women's History Month}

We have come a long way and still have a long way to go! Despite the progress, women still face more struggles between family and career. But looking into the future, time is on our side: gender equality is becoming more and more the norm and we are healthier, live longer, and can accomplish more.

Technology and computer science play increasingly important roles in today's world. I think it is important that more women get into the fields of science and technology; this is a way to make a good living and to have the chance to push the advancement of the world forward.

\section{Photo Credit}

Photo of Alice Chang courtesy of Sun-Yung Alice Chang. 


\section{Maria Chudnovsky}

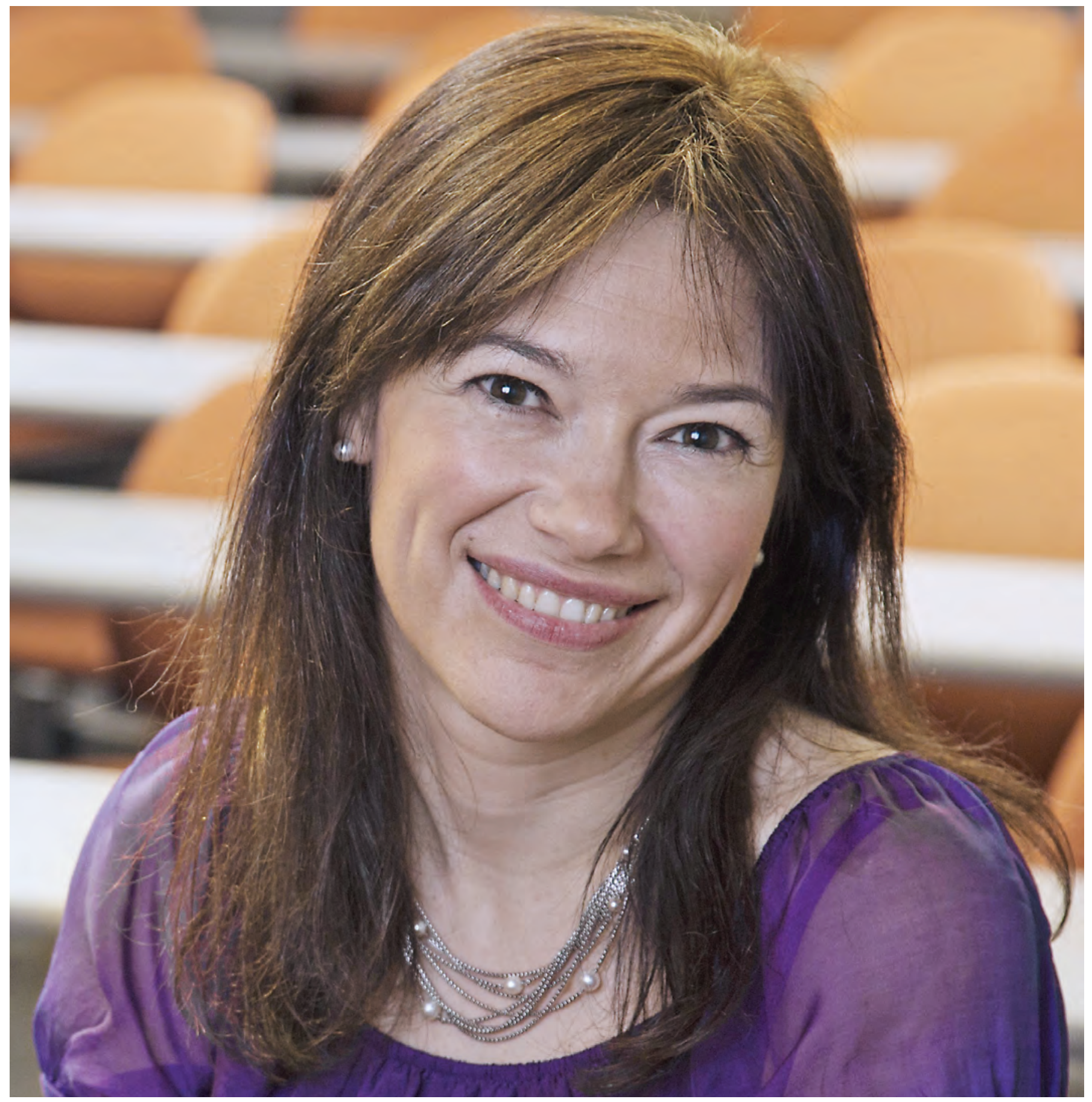




\section{WOMEN'S}

\section{HISTORY}

MONTH

\section{Synopsis of Research}

Maria Chudnovsky works in graph theory. She tries to understand the structure of graphs defined by certain properties, and then answer questions about these graphs using the structural information. As a graduate student, she was a part of a team of researchers that proved the Strong Perfect Graph Theorem, thus settling a forty-year-old conjecture, which proposed a certain characterization of graphs that behave especially nicely with respect to graph coloring. Since then she has continued to work on questions relating graph structure and coloring, studying both the theoretical and the algorithmic aspects of the problem. Chudnovsky is currently working on several exciting projects, such as determining the complexity of coloring a graph with various induced subgraphs excluded, trying to better understand the structure of perfect graphs, and more.

\section{Biography}

Maria Chudnovsky received her BA and her MSc from Technion, and her PhD from Princeton University in 2003. Currently she is professor at Princeton. Before returning to Princeton in 2015, she was Veblen Research Instructor at Princeton University and the IAS, assistant professor at Princeton, Clay Mathematics Institute Research Fellow, and Liu Family Professor of Industrial Engineering and Operations Research at Columbia University. Her research interests are in graph theory and combinatorics. She is an editorial board member of the Journal of Graph Theory. For her work on the Strong Perfect Graph Theorem, Chudnovsky was awarded the Ostrowski Foundation research stipend in 2003 and the prestigious Fulkerson Prize in 2009. She was also named one of the "brilliant ten" young scientists by Popular Science. In 2012 Chudnovsky received the MacArthur Foundation Fellowship. In 2014, she was an invited speaker at the International Congress of Mathematicians.

\section{Advice to Young Women}

Her advice to young women and everyone else is: Don't give up before you absolutely have to, or want to.

\section{Photo Credit}

Photo of Maria Chudnovsky courtesy of Maria Chudnovsky. 


\section{Carina Curto}

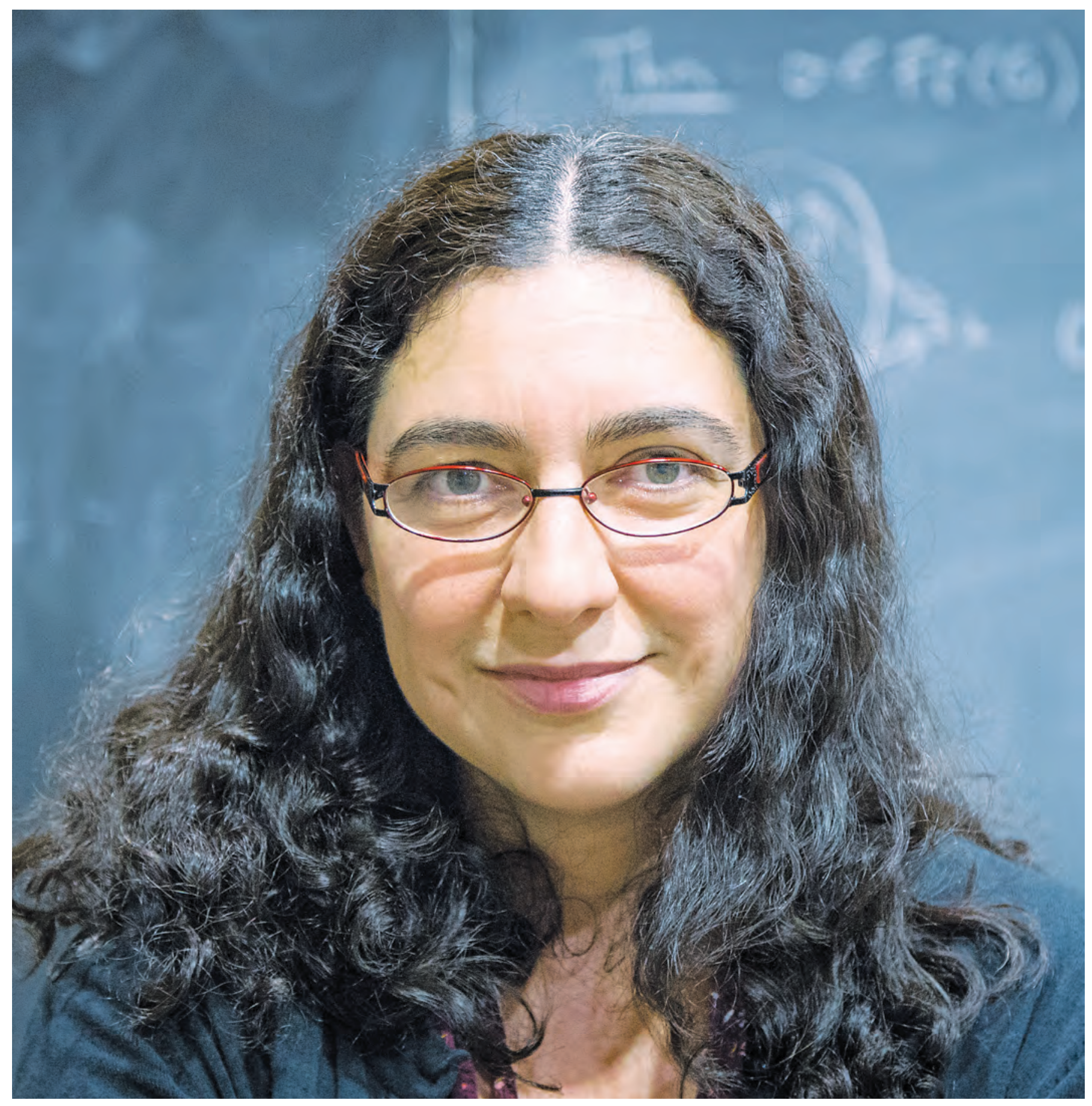




\section{WOMEN'S}

\section{HISTORY}

MONTH

\section{Synopsis of Research}

Carina Curto's research lies at the interface of neuroscience and mathematics. She is fascinated by fundamental questions in neuroscience, such as:

How do neurons encode and process information about the outside world?

How are memories stored and retrieved in neural networks, and what kinds of architectures underlie the rich and varied dynamics observed in the brain?

As a mathematician, Curto works on developing models that capture experimentally observed phenomena but are simple enough to admit theoretical analysis. She proves theorems about neural networks and neural codes and uses computer simulations to build intuition and play with examples. She also develops novel data analysis techniques to address the challenges that arise in working with biological data. Her collaborators include neuroscientists and mathematicians of different backgrounds. Many unexpected areas of mathematics have been useful in her work, ranging from applied algebra and topology to graph theory, dynamical systems, and discrete geometry. Curto hopes to eventually write a book on neural networks and neural codes, with an eye towards young mathematicians who are interested in applications and have a fondness for algebra, topology, and combinatorics.

\section{Biography}

I grew up in Iowa City, Iowa, which is home to the Iowa Writers' Workshop. As a child, I always thought I would either become a writer or a theoretical physicist. My father was a mathematician, so that was not glamorous enough for my childhood dreams. I also grew up speaking Spanish, and my parents instilled in me a strong sense of our Argentine background. When I was in high school, I studied everything that interested me, taking as many classes as I could at the University of Iowa. The state law at the time allowed high school students to take college credits for free in any subject not offered at their school. Most often, this was used for advanced math classes-but I milked it, adding courses in physics, economics, computer science, writing, French literature, and Italian. Somehow, I also found time to be a serious tennis player and member of the debate team.

My college years at Harvard were more focused, and it was there that I finally discovered my love for mathematics and its deep connections to physics. By the time I got to graduate school, I was on a path to becoming a string theorist. Halfway through my $\mathrm{PhD}$, however, I became disillusioned with string theory and fell in love with neuroscience. This sent my career on a very different path. After graduating, I spent three years as a postdoc in a neuroscience lab at Rutgers. It was an exhilarating experience: a complete cultural immersion into a world of science I had barely known existed when I first entered graduate school. Eventually, I returned to math as a mathematical neuroscientist. I'm currently a tenured math professor at Penn State University. I've been awarded a Sloan Research Fellowship and multiple grants from NSF and NIH. I live in State College with my husband-who is also a frequent collaborator-and our two kids.

\section{Advice to Young Women}

Dream big and make your dreams a serious priority! And don't worry too much about pleasing your family and mentors by "following the rules." Great research is like great art: it takes courage, and a conviction that what you do matters-even if others don't initially see the value.

Photo Credit

Photo of Carina Curto by Carley LaVelle, Penn State. 
Ingrid Daubechies

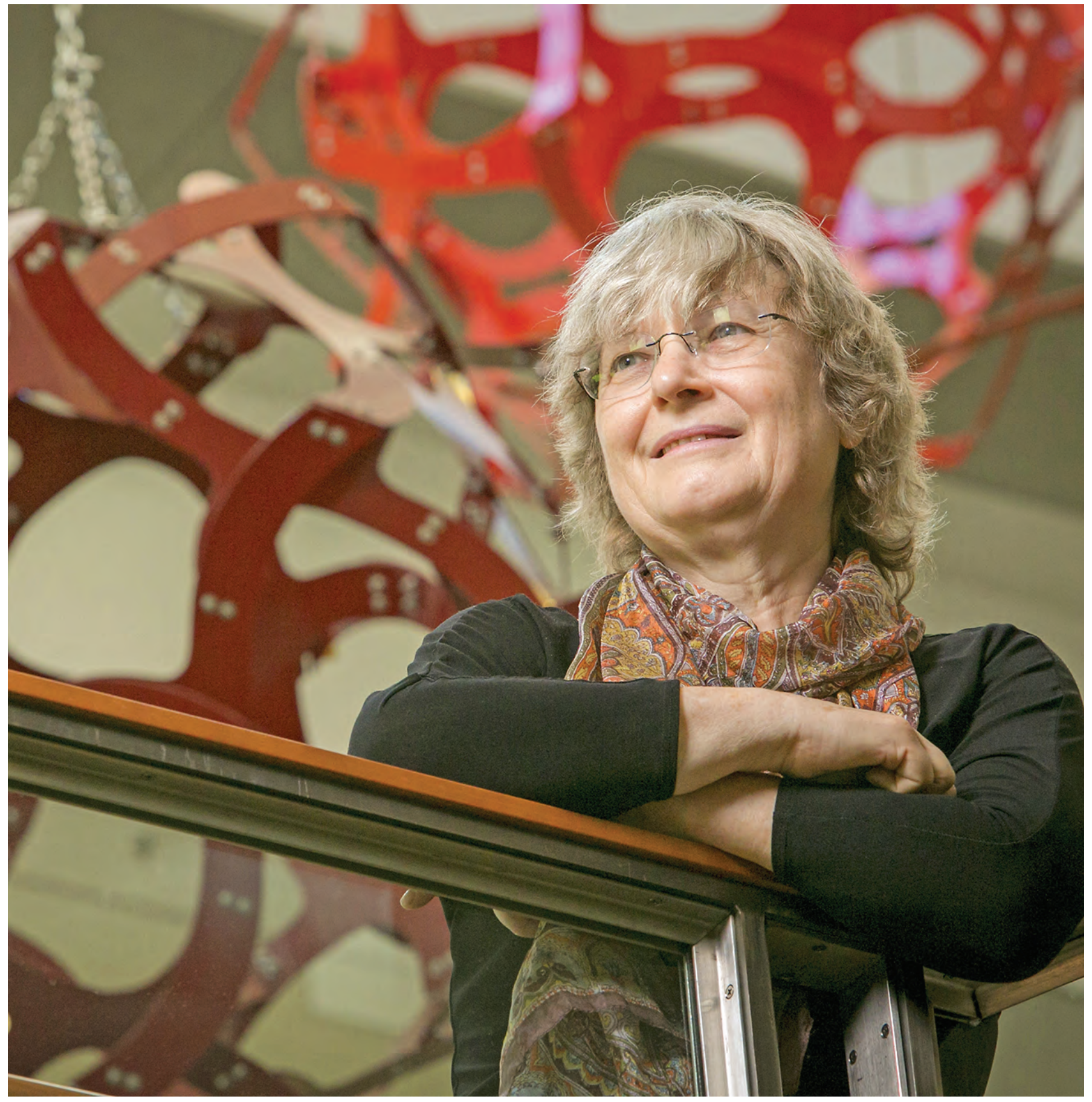


WOMEN'S

HISTORY

MONTH

\section{Synopsis of Research}

Ingrid Daubechies works on mathematics applied to the sciences and engineering, and beyond. Daubechies started out as a mathematical physicist, with both $\mathrm{BS}$ and $\mathrm{PhD}$ degrees in physics. She first worked on rigorous results in quantum mechanics, but then found that she was also interested in applying the mathematical techniques she had learned during her $\mathrm{PhD}$ training to other questions, more related to engineeringand presto-she became an applied mathematician.

Daubechies got interested in wavelets in the early 1980s. The wavelet transform had then just been formulated as an alternative, with very interesting properties, to the windowed Fourier transform (aka, coherent state representation in quantum mechanics) for the analysis of time-dependent signals. It turned out that wavelets were connected to many other developments in harmonic analysis, computer science, and electrical engineering, but that synthesis came later.

In 1987, Daubechies' work on wavelets led her to construct orthonormal wavelet bases for which the generating wavelet combined smoothness with being supported on an interval - a result that many had believed to be impossible. The construction was completely motivated by applications: other smooth wavelet bases all had infinite support and thus had to be truncated for numerical applications. Because she never believed that applications necessarily imply that mathematical elegance would be sullied or corrupted by implementation details, Daubechies had a very different approach to the construction, which turned out to work. This work is probably still the one for which Daubechies is best known; it certainly is the most cited.

Over the years, Daubechies has worked with scientists and engineers in many different fields, often but not always involving wavelets-typically on signal analysis or representation, sometimes combined with so-called inverse problems. These fields include neuroscience, geophysics, optics, biological morphology, medicine, and most recently art history. ${ }^{1}$

\section{Biography}

Ingrid Daubechies earned her $\mathrm{PhD}$ in theoretical physics from Vrije Universiteit Brussel. She is best known for her breakthroughs in wavelet research and her contributions to digital signal processing. Some of the wavelet bases she constructed have become a household name in signal analysis; they, and other computational techniques she and collaborators developed, have been incorporated into the JPEG2000 standard for image compression. Apart from her work on wavelets, Daubechies has contributed to other seminal advances in time-frequency analysis.

Her career has seen many impressive firsts: Daubechies was the first female full professor of mathematics at

\footnotetext{
${ }^{1}$ See "Reunited: Francescuccio Ghissi's St. John Altarpiece" in the January 2017 Notices www. ams . org/pub7ications/journa7s/ notices/201701/rnoti-p8.pdf.
}

Princeton, the first woman to receive the National Academy of Sciences Award in Mathematics in 2000, and the first woman president of the International Mathematical Union (2010-2014).

In addition to her commitment to educating and mentoring the next generation of mathematicians, Daubechies continues to break new ground in mathematics research and expand its impact outside of her discipline, focusing on the analysis of signals and inverse problems in a wide range of settings, with applications ranging from fMRI and geophysics to paleontology and the study of fine art paintings.

\section{Advice to Young Women}

In all my work, I have been motivated by trying to understand, in mathematical terms, what was going on. I believe mathematicians can be enormously valuable in interdisciplinary collaborations, because our mathematical training has given us tools for mathematically sophisticated frameworks and techniques that can really help in thinking in an abstractly well-structured way about complex questions in other fields. I have also learned not to underestimate the sophistication of thinking of my non-mathematical collaborators, even when they don't necessarily have the mathematical vocabulary or semantics to formulate what guides them. My job is, in part, to recognize and then unravel these trains of thought, a process that takes time, and that can only work if both parties are patient communicators. It is my job then to take them further with the appropriate mathematics. When I am lucky, the results are interesting mathematically and also feed back to the application in an interesting, non-trivial way. I have found this a fulfilling way to build a career, even if it is not quite conventional. Many of my students have found ways in making this work for their careers as well, in a diversity of directions that I find very gratifying.

I would encourage any young mathematician thinking of working on applications to learn as much pure mathematics as possible, limited only by appetite and time, not by how applicable they think it might be. I believe that I have used in my own career all the mathematics I understand, I just wish I knew more, and that I still had the time to learn more. On the other hand, I have also found that great joy comes from working with others. Experiencing the give-and-take of half-baked ideas that then are given substance through the collaborative effort is exhilarating.

\section{Photo Credit}

Photo of Ingrid Daubechies courtesy of Les Todd: Duke Photography. 
Irene Fonseca

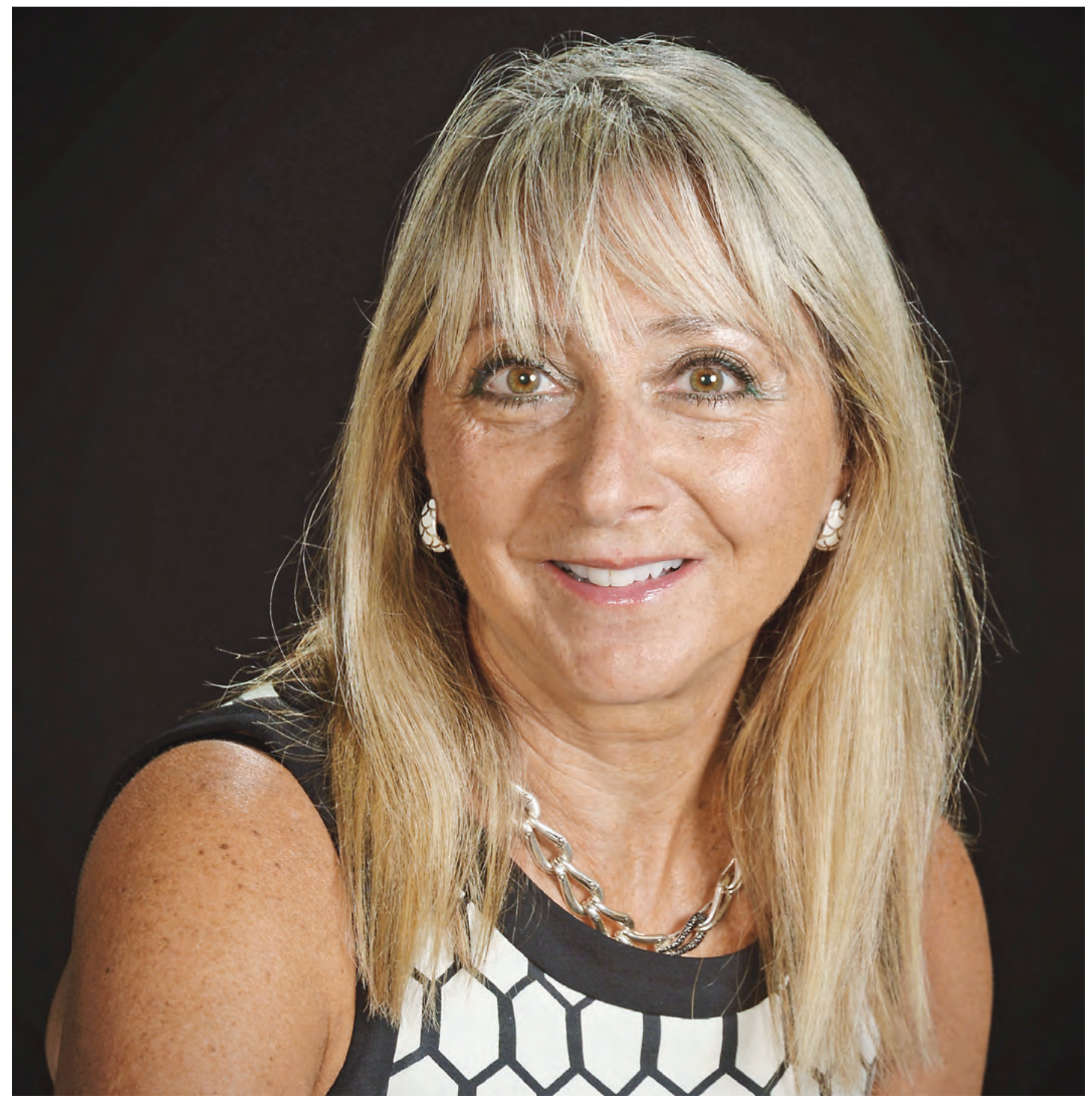


WOMENS

\section{HISTORY}

MONTH

\section{Synopsis of Research}

Irene Fonseca's main contributions have been in the variational study of ferroelectric and magnetic materials, composites, thin structures, phase transitions, and in the mathematical analysis of image segmentation, denoising, detexturing, registration, and recolorization in computer vision. Her research program continues to explore modern methods in the calculus of variations motivated by problems arising from materials science and imaging science.

\section{Biography}

Irene Fonseca has supervised $14 \mathrm{PhD}$ students and mentored 36 postdoctoral fellows. She is a Fellow of the American Mathematical Society (AMS) and a Fellow of the Society for Industrial and Applied Mathematics (SIAM). Fonseca was SIAM president in 2013 and 2014. She is a Grand Officer of the Military Order of Saint James of the Sword (Grande Oficial da Ordem Militar de Sant'Iago da Espada, Portuguese Decoration). She serves on fifteen editorial boards, including Advances in Calculus of Variations, ESAIM:COCV (SMAI), Journal of Nonlinear Science, Le Journal de l'Ecole Polytechnique, M3AS, and SIAM Journal on Mathematical Analysis. She is a member of several advisory and scientific boards of research centers and institutes, including the IMA and SAMSI; participates in international prize committees; and is on review and evaluation panels of multiple universities in the US and abroad.

\section{Advice to Young Women}

- Find a good advisor/mentor who has availability to see you periodically and frequently.

- Get involved in activities that will allow you to network with other students in math-related fields, such as math clubs and SIAM student chapters.

- Go to workshops, conferences, summer schools, and when appropriate showcase your work through posters, contributed talks, etc.

- Have fun doing mathematics. Let it be a passion.

- Be confident in your math skills, don't hesitate to ask questions, and be assertive when you think you know the answer.

\section{Photo Credit}

Photo of Irene Fonseca courtesy of Irene Fonseca. 


\section{Carolyn Gordon}

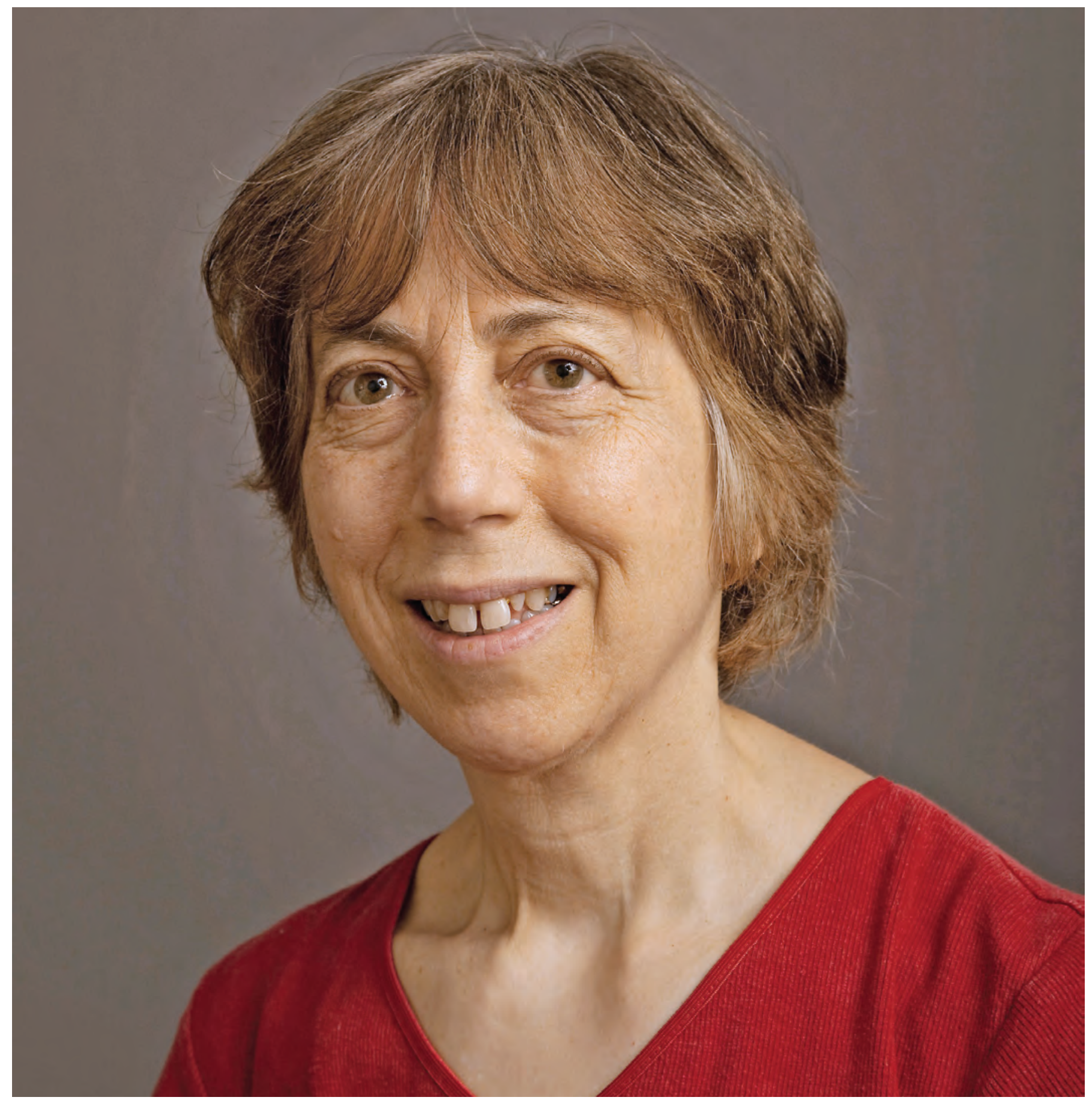




\section{WOMEN'S}

\section{HISTORY}

MONTH

\section{Synopsis of Research}

Carolyn Gordon's research is in the area of Riemannian geometry, focusing on inverse spectral problems and on homogeneous spaces. Inverse spectral problems ask the extent to which geometric or topological properties of an object such as a vibrating membrane or, more generally, a Riemannian manifold are encoded in spectral data. For example, Gordon, along with David Webb and Scott Wolpert, gave a negative answer to Mark Kac's question, "Can one hear the shape of a drum?"

\section{Biography}

When I was growing up, it was assumed that women would go to college primarily to get their "Mrs Degree." I never considered an academic career-or any career for that matter-until my undergraduate algebra professor, Stephen Piper, asked me if I had plans for graduate school. Just the question itself both ignited a dream and helped give me the confidence to pursue it. Soon after, I entered a $\mathrm{PhD}$ program at Washington University, later returning as a faculty member. I was fortunate to have my twobody problem solved before it existed. I met my husband David Webb when he also began a tenure-track position at Washington University. A few years later we moved to Dartmouth College. My greatest joy has been parenting our daughter Annalisa. She was often my mentor as well as my child, especially when I stretched well beyond my comfort zone in taking on the presidency of the AWM during her elementary school years. Some honors (mostly traceable back to a ten-minute contributed talk at a regional AMS meeting early in my career) have included a Chauvenet Prize with co-author David Webb, an endowed professorship, and becoming a Fellow of the AMS and of the AAAS. Perhaps the honor (if not the title!) that makes me most proud is a Vagina Warrior Award for mentoring, as it was presented by students.

\section{Advice to Young Women}

My first suggestion to younger women is to take every opportunity to present your work and talk to other mathematicians. You never know when a mentor may appear. Indeed, my interest in spectral geometry arose from a suggestion by Rich Millman, a member of the tiny audience during that early-career ten-minute talk at a regional AMS meeting. His comments not only changed my research trajectory but, as a consequence, opened many new career opportunities for me. Secondly, while I've "known" throughout my career that I'm an impostor, I've come to realize that if I haven't been found out yet, I probably never will be! So if you too feel like an impostor, know that you're not alone and just keep going!

\section{Comments on Women's History Month}

I'm delighted to see this celebration of Women's History Month in the Notices and honored to be included. My generation of women has had many more opportunities than those before us, and we've seen considerable progress in our lifetimes. In the current national climate, it is essential to remember our history and ensure that we continue to move forward.

\section{Photo Credit}

Photo of Carolyn Gordon courtesy of Dartmouth College / Joseph Mehling. 


\section{Fan Chung Graham}

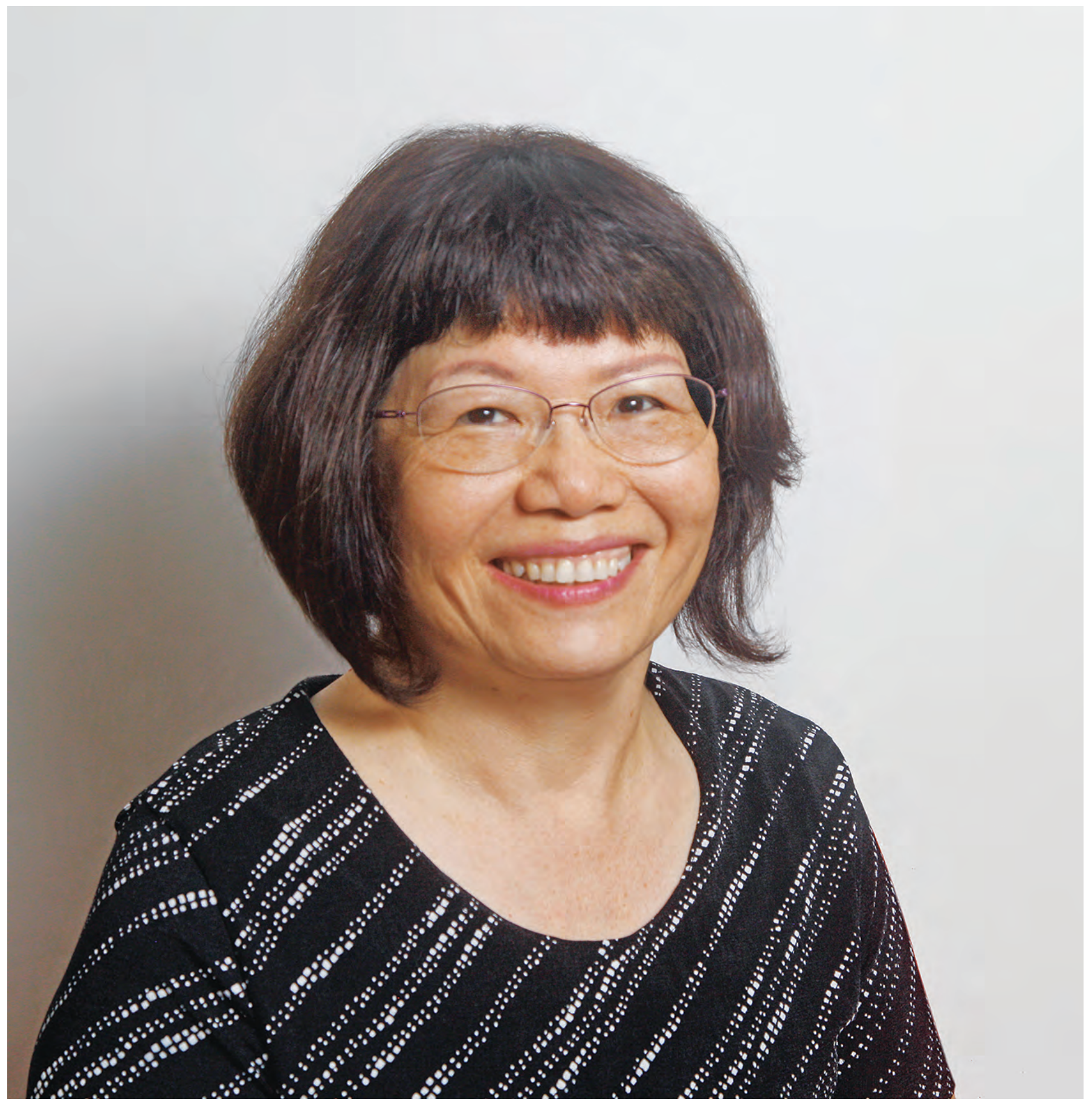




\section{WOMEN'S}

HISTORY

MONTH

\section{Synopsis of Research}

Fan Chung Graham's research interests are primarily in graph theory, combinatorics, and algorithmic analysis. She specializes in spectral graph theory, extremal graphs, the theory of quasi-randomness, and the probabilistic analysis of complex networks. Recently her work has included local graph algorithms for complex networks and random walks-based ranking algorithms.

Graham has made fundamental contributions in the following areas of mathematics:

- Spectral graph theory: Her research has been instrumental in establishing the foundations of spectral graph theory, introducing the normalized Laplacian, and establishing the basic relationship between the Laplacian spectral and numerous properties of graphs with general degree distributions.

- Random graph theory and complex networks: She has created a theory for treating random graphs that have arbitrary degree distributions, including power law graphs. This allowed her to analyze the behavior of the ubiquitous power law network in greater detail than has ever been possible previously.

- Quasi-random graphs: Her major contribution is the introduction and development of the concept of quasi-randomness. In a series of research papers with several co-authors, a large family of graph properties were shown to be equivalent. The set of equivalent quasi-random properties is surprisingly diverse and has been a seminal driver in the development of graph limits.

- Unavoidable graphs and universal graphs: A basic question in extremal graph theory is to find unavoidable patterns and structures in graphs with given density or distribution. A complementary problem is to find a smallest graph which contains every member of a given family of graphs as subgraphs. In work with Paul Erdős, she has determined the sizes and structures of unavoidable graphs and hypergraphs. Also, with several authors, she derives many elegant and surprising results on universal graphs. Her fundamental contributions in these areas of extremal graph theory have many applications in parallel computations.

- Ranking algorithms and clusters analysis in graphs: In recent years, she has done seminal work on the quantitative analysis for ranking algorithms in graphs. Instead of using usual graph distances, which does not work well for numerous information networks with the trait of small-world phenomenon, she introduced the mathematical analysis of quantitative ranking based on random walks. Her research on graph invariants derived from ranking has far-reaching impact not only in search algorithms and clustering analysis but also in the study of the geometry of networks.

\section{Biography}

Fan Chung Graham is a Distinguished Professor of Mathematics and professor of computer science and engineering at the University of California, San Diego. She is also the Paul Erdős Professor in Combinatorics.

Graham was awarded the Allendoerfer Award by the Mathematical Association of America in 1990. She is a member of the American Academy of Arts and Sciences, and she is an academician of Academia Sinica. She is a Fellow of the American Mathematical Society and of SIAM.

\section{Advice to Young Women}

Don't be intimidated!

I have seen many people get discouraged because they see mathematics as full of deep incomprehensible theories. There is no reason to feel that way.

In mathematics whatever you learn is yours and you build it up-one step at a time. It's not like a real-time game of winning and losing. You win if you benefit from the power, rigor, and beauty of mathematics. It is a big win if you discover a new principle or solve a tough problem.

\section{Photo Credit}

Photo of Fan Chung Graham by Ché Graham. 


\section{Rosemary Guzman}

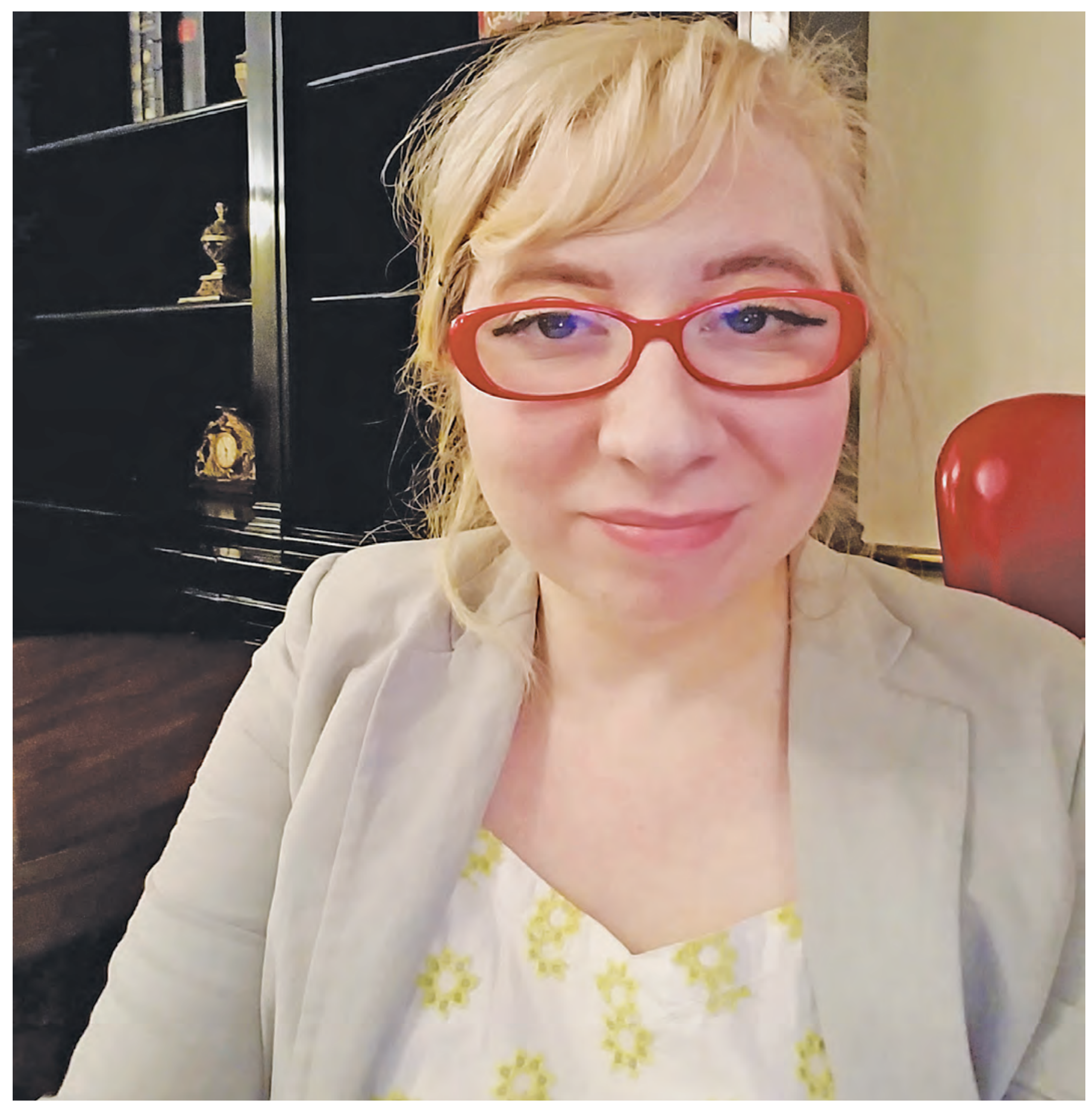




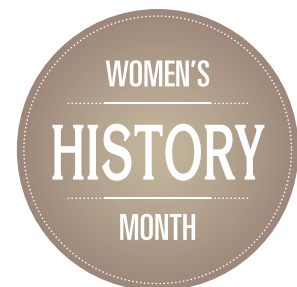

\section{Synopsis of Research}

Rosemary Guzman likes to explore questions in different areas of mathematics from a topological viewpoint. Her research interests are in hyperbolic 3-manifolds and geometric group theory, but co-advising several undergraduate research projects with her colleague Ruth Davidson-one in phylogenetics and another related to discrete Morse theory and persistence homology-provided rich and fulfilling questions that benefited from topological insights. Currently, Guzman has an ongoing project with Peter Shalen, which aims to extend for certain hyperbolic 3-manifolds some of the geometric-topological results previously developed by Shalen and Marc Culler. Guzman has enjoyed diversifying her research program in this way; some questions are long and hard, and others are a bit more attainable and also accessible to undergraduates. Being able to continue working and learning with experts as well as mentor and advise younger students has been constructive, both mathematically and personally.

\section{Biography}

I am currently an American Academy of University Women (AAUW) American Postdoctoral Fellow at the University of Chicago, with Benson Farb as my mentor. My previous NSF Alliance Postdoctoral fellowship at the University of Iowa afforded me the opportunity, thanks to a progressive chair, Dan Anderson, to teach an undergraduate research course that proved successful, an experience contributing to my becoming assistant director of the Illinois Geometry Lab. One of the most inspiring moments as a mentor came when a former calculus student at the University of Illinois asked me to serve as her academic sponsor for a technology entrepreneurship class, where she worked on the first project of her company, YouMatter Studios.

I still remain in touch with past students, some of whom are now graduate students in mathematics. A major highlight of my time at Iowa was a recognition provided by the graduating class of 2014: I was named a professor who had imparted a positive difference in students' lives. This was truly special because, as a first generation Mexican American having a vision impairment and raised in public housing in Chicago, I am now granted the chance to guide others who are on the pathway to academia as the first in their families. Socioeconomics can be an even more sensitive topic than gender or ethnicity, and it presents complex but hidden challenges. For instance, my early education was at a Chicago public school named by then-Secretary of Education William Bennett as an example of "the worst school in America." Losing a parent to a terminal illness paused my work for a time, but this loss evoked the willingness of others, in this case my PhD advisor, to take a chance on someone who experienced an interruption. While the conventional path to academia may seem narrowly defined, women and anyone traversing this path non-traditionally should be fearless to seek those who are willing to invest in, and help grow, their talent.

\section{Advice to Young Women}

For younger women in mathematics, my advice is to form your networks with intent, understanding that the formation of different kinds of networks-research, mentoring, and teaching - serve distinct purposes, but all can uniquely contribute to the advancement of your career, especially where they overlap. From networks blossom communities, and for me, these are communities of likeminded friends and mathematicians: Project NExTers, the AWM community, the IAS Women and Math Program, the Math Alliance, USTARS, the National Center for Faculty Diversity, SACNAS, etc.

\section{Comments on Women's History Month}

When returning from a conference and sharing with my daughter a narration of my travel, I find that the pictures of me and other women mathematicians-some the pioneers of a past generation and some the pioneers of a newer generation today-provide an empowering reminder of the preeminent tradition of women as actualizers of mathematics. It is a contemporary history in which we may all take pride.

The best homage to Women's History Month is to simply stop and think (at your next conference, take a moment to pause and examine who is represented), ask questions (inquire of conference organizers whether they have childcare funds available-or will with their next grant), become aware (of programs like EDGE and MSRI-Up, for which a simple recommendation to a young female student can be transformative), learn from the voices of women at all stages (a graduate student or a faculty member may co-organize a panel on the intersectionality of gender, math, and diversity at JMM or within your own institution), connect (check in with new female graduate students and faculty), and support good causes and people that do the same.

Photo Credit

Photo of Rosemary Guzman courtesy of Rosemary Guzman. 


\section{Tara S. Holm}

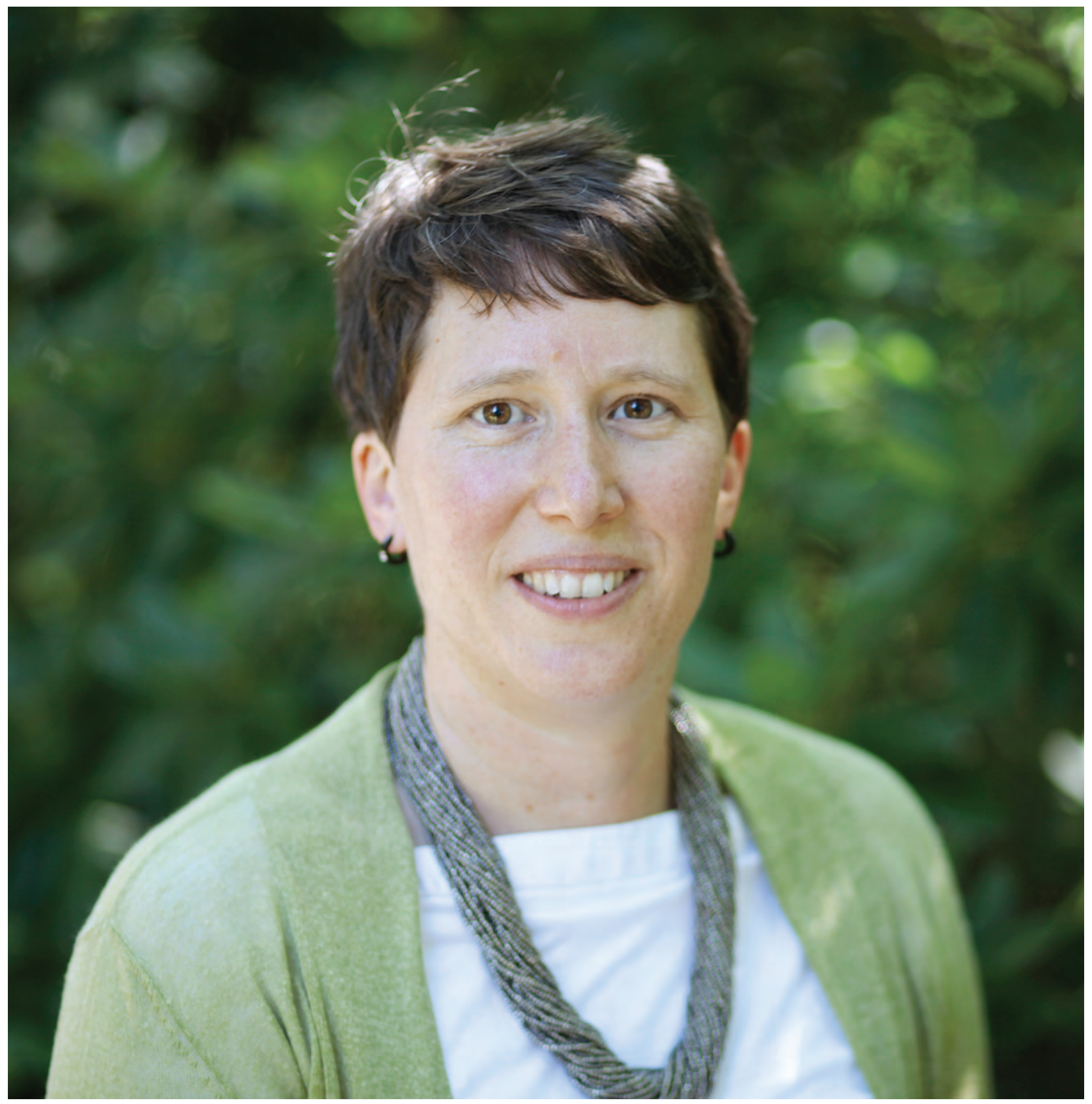


WOMEN'S

HISTORY

MONTH

\section{Synopsis of Research}

Tara S. Holm studies questions that originate in symplectic geometry but whose answers often involve methods from and have applications to algebraic geometry, algebraic topology, and combinatorics. Her principal focus is on group actions and quotients in symplectic geometry. Most recently she has made a foray into the developing area of quantitative symplectic geometry, analyzing the surprising new connections between symplectic geometry and number theory that arise when studying how one symplectic manifold can fit inside another. For embeddings of ellipsoids into toric surfaces, the question boils down to proving that a certain sum of two functions always has a positive output.

Holm has published over two dozen papers with nearly two dozen collaborators from half a dozen countries. She has advised two $\mathrm{PhD}$ students with more in the pipeline. Holm also has a deep passion for effective communication and undergraduate education. She envisions a world where all undergraduate students learn to recognize the beauty and power of mathematics.

\section{Biography}

Tara S. Holm is professor of mathematics at Cornell University. She earned her undergraduate degree at Dartmouth College and her PhD at MIT. She was an NSF postdoctoral research fellow at UC Berkeley and had been a faculty member at the University of Connecticut before arriving at Cornell. Holm has played leadership roles nationally with the American Mathematical Society; the Association for Women in Mathematics; Transforming Post-Secondary Education in Mathematics (TPSEMath.org); and Pro Mathematica Arte, the non-profit corporation that runs study-abroad programs for North American mathematics students in Budapest. She gives invited lectures around the world, including an AMS-MAA Invited Address at MathFest. Her research has been supported by grants from the National Science Foundation and the Simons Foundation. She is a Fellow of the American Mathematical Society and a 2013 Simons Fellow in Mathematics. At Cornell, she has been honored with a 2016 Mathematics Department Teaching Award and a 2017 Cornell Cook Award for improving the climate for women at Cornell. In addition to mathematics, she enjoys gardening, cooking with her family, and exploring the Finger Lakes.

\section{Advice to Young Women}

To young women entering the field, she would advise:

- Find collaborators, especially ones who are close enough to your field so that you can talk but far enough that they will teach you some new mathematics.

- Assume good intentions on the part of others. This helps smooth over most misunderstandings and may even turn adversaries into allies.

- Spend as much money as you can afford on the aspects of life that bring you the least joy (for Holm, that's laundry and house cleaning!). The time-for-money tradeoff is always worth it.

\section{Comments on Women's History Month}

On the occasion of Women's History Month, I have deep gratitude for the many senior women mathematicians who have made it easier for the younger generations to find their niche in the field. I have had the good fortune of many female role models and mentors whose encouragement carried me through some of the tougher moments of my career. I feel very lucky to have found a supportive network of collaborators and a job in an incredibly friendly and collegial department.

\section{Photo Credit}

Photo of Tara S. Holm by Melissa Totman. 
Olga Holtz

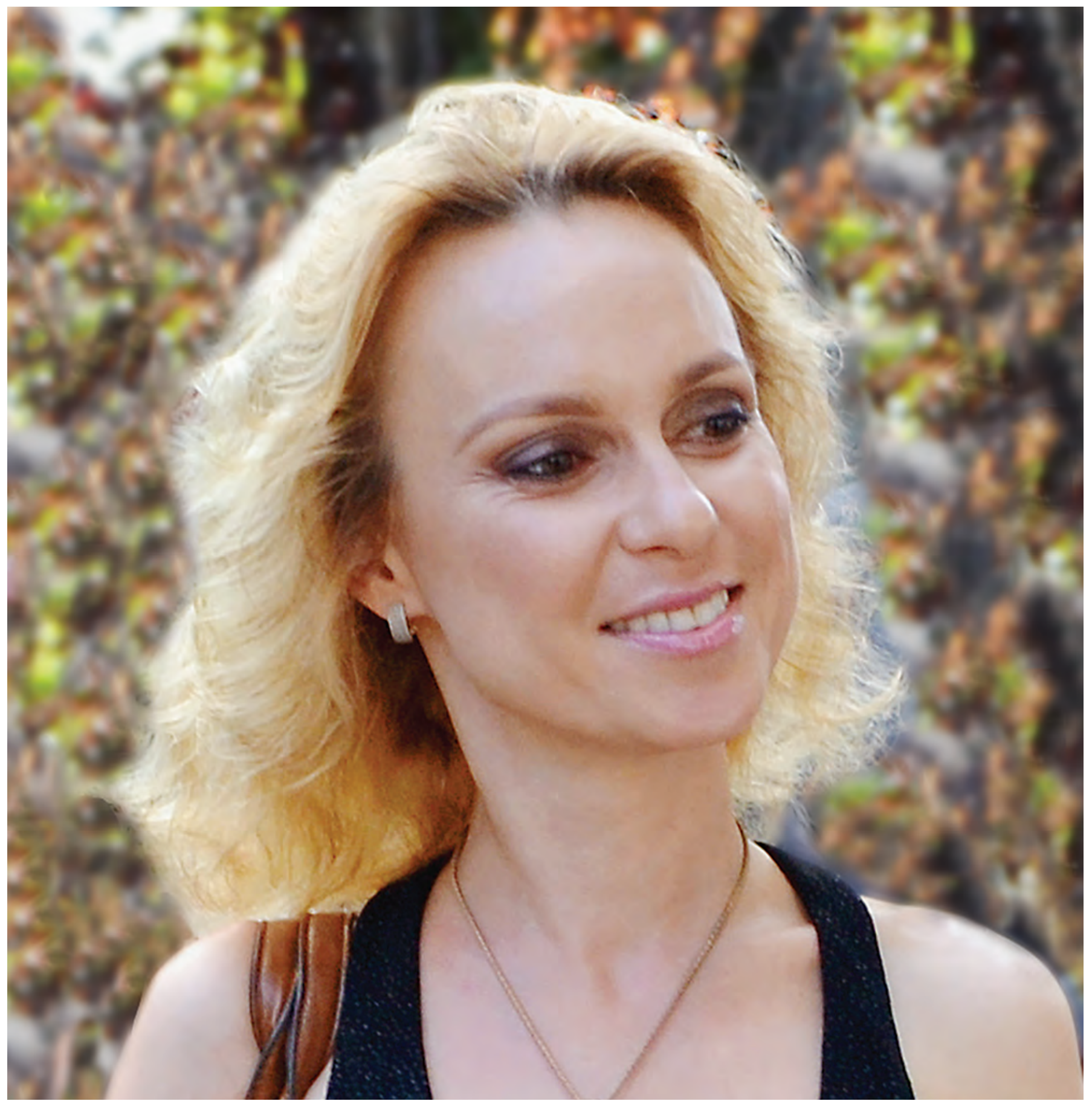


WOMEN'S

\section{HISTORY}

MONTH

\section{Synopsis of Research}

Olga Holtz's research interests include numerical analysis, parallel computing, complexity of algorithms, classical analysis, matrix and operator theory, approximation theory, orthogonal polynomials, commutative algebra, and enumerative combinatorics. Her main research contributions include solutions to several open problems in matrix theory, development of zonotopal algebra, building a framework for communication complexity of algorithms, and novel fast and stable parallel computation methods. In the future she hopes to complete a monograph on the Laguerre-Pólya class, a fascinating class of entire functions that appear in classical analysis, number theory, combinatorics, and statistical physics.

\section{Biography}

Olga Holtz received her diploma in applied mathematics from Southern Ural State University in Chelyabinsk, Russia, and her $\mathrm{PhD}$ in mathematics from the University of Wisconsin under the guidance of Hans Schneider.

She held a postdoctorate research position at the computer science department of the University of Wisconsin-Madison, a Humboldt Fellowship at the Institute of Mathematics of Technical University Berlin, and a Morrey assistant and an associate professorship at the Department of Mathematics of the University of California Berkeley.

Currently, Holtz is a professor of mathematics at the University of California Berkeley and a visiting professor of applied mathematics at Technical University Berlin and Berlin Mathematical School.

She has received a Sofja Kovalevskaja award, a European Mathematical Society Prize, an ERC Starting Grant, two von Neumann Fellowships at the Institute for Advanced Study, and an AMS Fellowship.

Holtz is also an award-winning screenwriter and film director.

\section{Advice to Young Women}

Dare to take risks in your career.

\section{Photo Credit}

Photo of Olga Holtz by Hamid Shafiezadeh. 


\section{Fern Y. Hunt}

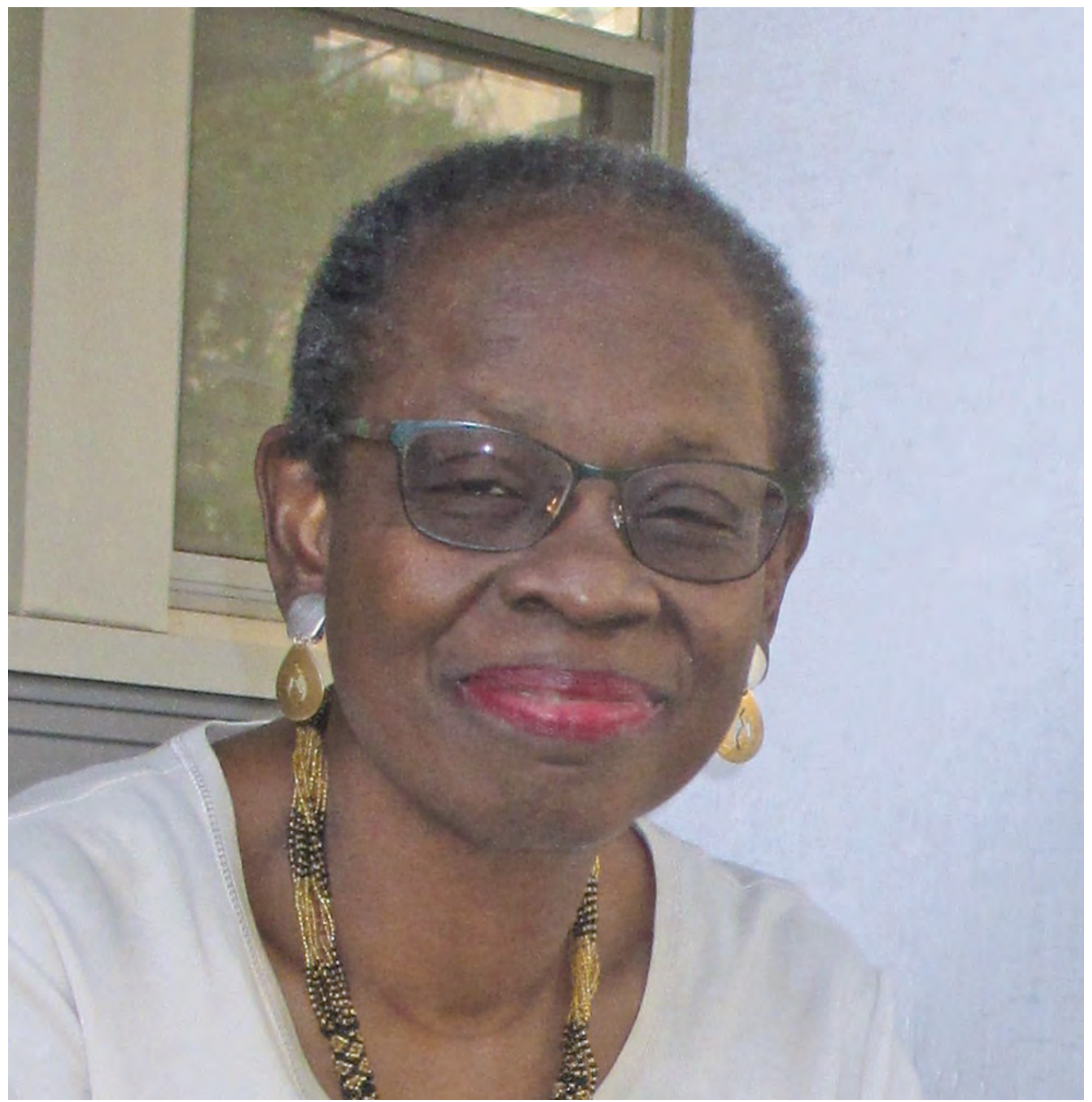


WOMEN'S

HISTORY

MONTH

\section{Synopsis of Research}

At NYU's Courant Institute, Fern Y. Hunt received a graduate education that blended pure and applied mathematics. Thus it was easier to consider working on problems that contributed meaningful insights into real-world problems while discovering interesting new mathematics. The result was a hybrid career of government and university involvement. Two examples illustrate what government research is like and how it might differ from what happens in an academic department. While at the Laboratory of Mathematical Biology at the National Institutes of Health, Hunt read an internal newsletter that reported the work of NIH researchers Loretta Leive and Robert Goldman. They studied the molecular chains that comprise the outer coating of gram negative bacteria. The coatings consist of chains of carbohydrate molecules of varying lengths that at once affect the degree of virulence of infection and play an important role in helping bacteria evade the immune systems of their hosts. Hunt met with them and after some eager initial discussion, a collaboration began. Using a simple Markov chain model of chain synthesis, they were able to offer a simple explanation for the data patterns Leive and Goldman observed based on a preference for the synthesis of certain chain lengths. This preference persisted even in mutant bacteria that had decreased capacity to produce single chain units.

The second example had an unusual beginning. Out of curiosity, Hunt attended what turned out to be an excellent lecture on clustering in random sequences by a senior (non-mathematician) scientist from the Glidden Paint company. There she met Jonathan Martin, NIST engineer and head of a group that studied degradation of painted and coated surfaces. Sometime later Hunt had a lunchtime chat with then division colleague Holly Rushmeier (now a professor of computer science at Yale University) about computer graphic rendering. That discussion led to a collaboration with other NIST scientists and university researchers on a project that demonstrated that radiometrically accurate but visually realistic computer-based renderings of appearance aspects of coated surfaces could be produced. This work was a proof-of-concept of what Hunt first thought was rather a quirky vision of Martin'sthat the standards governing the appearance of coated surfaces devised by committees of human industry and government experts could be enhanced or even replaced by computers that pass the Turing test, playing a visual "Imitation Game."

Hunt has also conducted research on the discrete approximation of dynamical systems and applications of Markov chains. More information on her research can be found in MathSciNet ${ }^{\circledR}$ or in the Web of Science database (under "FY Hunt").

\section{Biography}

Fern Y. Hunt is a New York City native. She graduated from Bryn Mawr College with a BA degree in mathematics and from the Courant Institute of Mathematical Sciences with a PhD in 1978. Later that year Hunt joined the faculty of Howard University's mathematics department, where she received an NSF Career Award, other NSF grants, Howard University research grants, and an Association for Women in Mathematics travel grant. In 1991, Hunt took a full-time position at the National Institute of Standards and Technology. In 1999, Hunt received the Arthur S. Flemming Award for outstanding federal service in science. Hunt has also appeared in the modern Leonardos exhibit at the Chicago Museum of Science and Industry since 2006. She has served on several committees, notably the NSF Committee of Visitors 2016, the organizing committee of the Infinite Possibilities Conference 2014-2015, the executive committee for AWM in 2002-2006, and the first Association for Women in Mathematics Olga Taussky Todd Award Committee in 2006. Hunt has also served on the Bryn Mawr College Board of Trustees and been an emerita member since 2009.

\section{Advice to Young Women}

If you are a recent $\mathrm{PhD}$ or you have just begun research in a new subfield, I would recommend attending as many research conferences as time and money allow. If this is not practicable, make use of online resources such as the arXiv and MathSciNet and read the blogs of outstanding mathematicians in your area. If you have written several papers on a particular topic, organize a special session at an annual conference and invite speakers working in your field. This is a good way to stay abreast of what is happening and identify possible collaborators. Like many mathematicians I am quite introverted; but as a woman of color, I often had to operate way out of my comfort zone. You cannot be isolated and be successful.

\section{Photo Credit}

Photo of Fern Y. Hunt courtesy of Fern Y. Hunt. 


\section{Trachette L. Jackson}

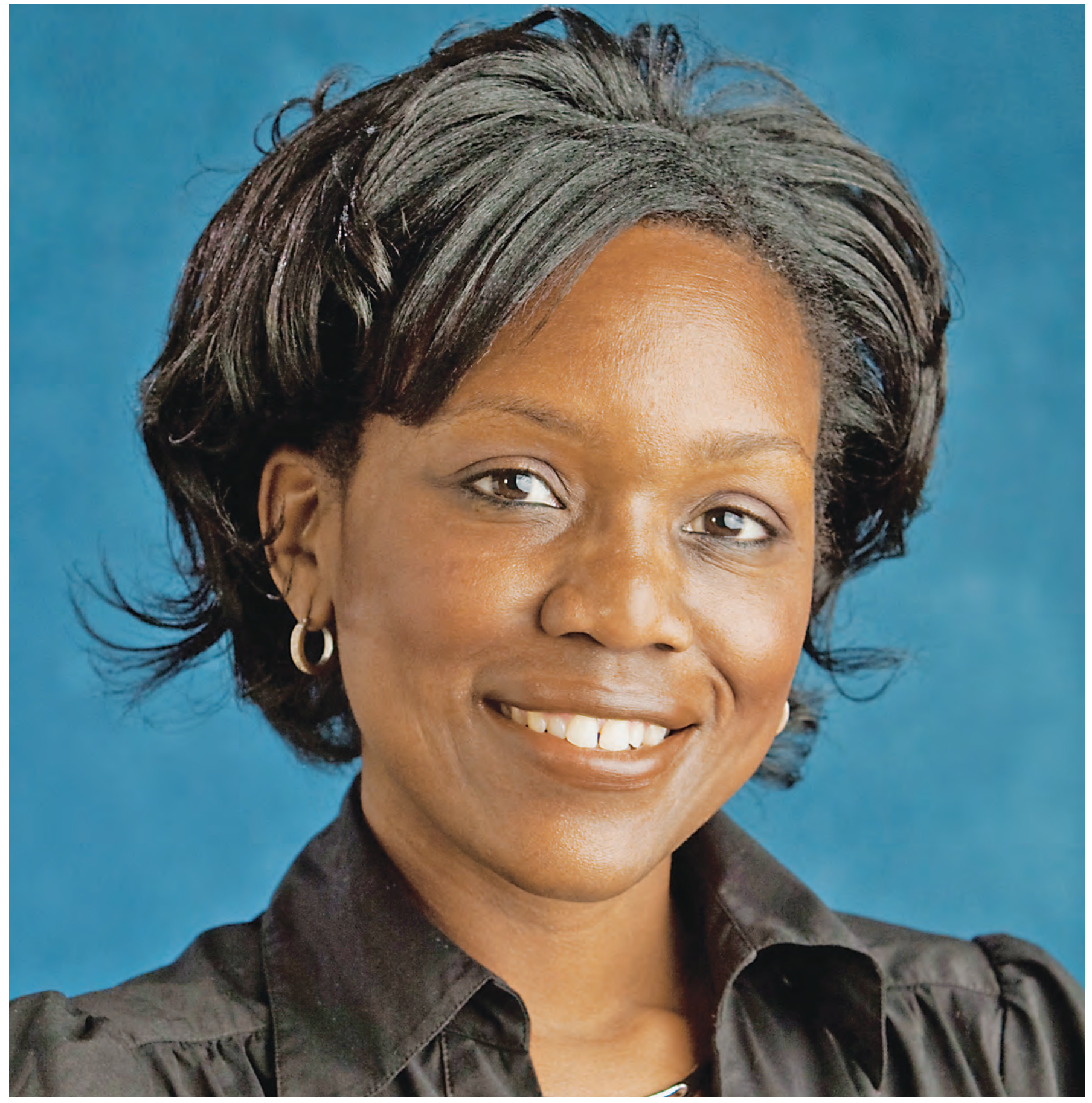


WOMEN'S

HISTORY

MONTH

\section{Synopsis of Research}

Trachette L. Jackson's research lies within the overarching field of mathematical biology, which can be characterized by the use of sophisticated mathematical, statistical, and computational modeling techniques in order to facilitate a deep understanding of biology, for human benefit and the mitigation of human harm. Within mathematical biology she specializes in computational cancer research or mathematical oncology. It is an exciting time to work in this interdisciplinary field. Even TIME $^{\circledR}$ Magazine (April 2013) agrees that "a team-based, cross disciplinary approach to cancer research is upending tradition and delivering results faster." Mathematical and computational modeling approaches have been applied to every aspect of tumor growth, from mutation acquisition and tumorigenesis to metastasis and treatment response. Jackson's research focuses on developing mathematical approaches that are able to address critical questions associated with vascular tumor progression and targeted therapeutics.

\section{Biography}

Trachette L. Jackson earned her PhD in applied mathematics in 1998 from the University of Washington under the direction of James D. Murray, one of the founding leaders in the field of mathematical biology. She conducted postdoctoral research at the Institute for Mathematics and its Applications and at Duke University. She is currently full professor of mathematics at the University of Michigan. Jackson is an award-winning educator and scholar whose research in mathematical oncology has received international attention. In 2003, she became the second African American woman to receive the prestigious Alfred P. Sloan Research Award in Mathematics; in 2005 she received the James S. McDonnell 21st Century Scientist Award; in 2008 Diverse Magazine honored her as one of the year's Emerging Scholars. In 2010 she received the Blackwell-Tapia Prize, which recognizes a mathematician who has contributed significantly to research and who has contributed in significant ways to addressing the problem of underrepresentation of minorities in math.

Jackson's commitment to theoretical and computational cancer research is evidenced by her joint effort with Sophia Merajver to spearhead a Quantitative Oncology subsection in the authoritative journal Cancer Research, for which she now serves as senior editor. Jackson has built her career on collaborative research and educational activities that cut across traditional disciplinary boundaries and envisions that this type of research will eventually change the face of cancer research.

In order to address the national challenge of educating and training a diverse scientific workforce capable of unifying the fields of mathematics and the natural sciences, Jackson developed and directs the Applied and Interdisciplinary Mathematics Bridge to the PhD program at the University of Michigan. The long-term goal of the Bridge program is to prepare the next generation of under-repre- sented minority scholars to combine a deep knowledge of applied science with the mathematical, computational, and physical sophistication needed to address the increasingly complex problems on the horizon and to become leaders in the international scientific community.

\section{Advice to Young Women}

Be confident in your abilities, and don't let anyone dissuade you from pursuing your passion. Find a group of like-minded peers and a more senior mentor who can provide you with guidance and support.

\section{Photo Credit}

Photo of Trachette L. Jackson courtesy of Trachette L. Jackson. 


\section{Dusa McDuff}

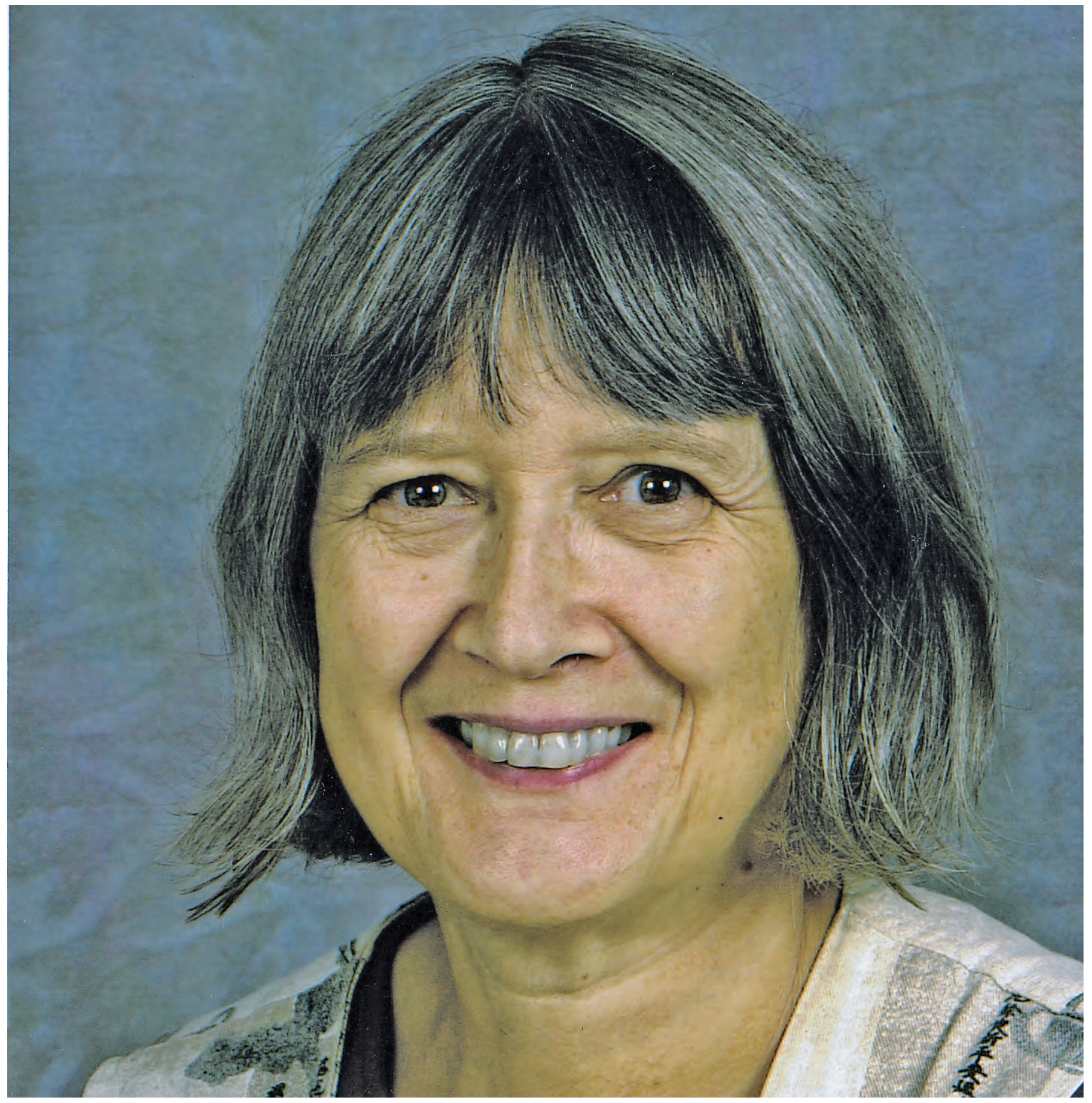




\section{WOMEN'S}

\section{HISTORY}

MONTH

\section{Synopsis of Research}

Dusa McDuff started her career in functional analysis, but after studying for six months with Gel'fand in Moscow, moved into algebraic topology. After completing what was essentially a second $\mathrm{PhD}$ with Graeme Segal, McDuff became interested in understanding the connections between the classifying spaces for foliations and diffeomorphisms, which gradually led her to study the group of symplectic diffeomorphisms. At that time, in the beginning and mid1980s, symplectic geometry was revitalized by several new insights, particularly by Gromov's introduction of J-holomorphic curves, and McDuff has stayed in that area of mathematics ever since. She used Gromov's theory to understand the properties of the symplectic blow up and blow-down construction (a variant of the construction in complex geometry) and to classify the simplest symplectic 4-manifolds, namely the rational and ruled manifolds. With Lalonde, McDuff spent many years trying to understand how to deform symplectic balls in dimension 4 and higher in order to explain the relation between ball embeddings and so-called displacement energy (which is a dynamic concept). More recently, McDuff has spent much time, both on her own and with Felix Schlenk and other collaborators, figuring out how to embed symplectic ellipsoids into each other and undertook a joint project with Katrin Wehrheim to clarify various foundational issues in the field of symplectic topology. McDuff has also written two textbooks with Dietmar Salamon.

\section{Biography}

I grew up in Edinburgh, Scotland, just after the end of the Second World War in an academic English family. I went to a girls' school and studied the cello at the wonderful Waddell School of Music. However, I found mathematics more compelling than cello playing and decided at an early age to become a mathematician, an academic career being the only kind of career that was valued in our house. I stayed in Edinburgh for my undergraduate work, going to Cambridge for my PhD. After two post-doc years in Cambridge, I was a lecturer in York and then Warwick, spent a transformative year at MIT in 1974-5, and went to Stony Brook University in 1978. Since 2007 I have been Helen Lyttle Kimmel '41 Professor of Mathematics at Barnard College. I was elected a Fellow of the Royal Society in 1994 and a member of the National Academy of Sciences in 1999. I was awarded the AMS Satter Prize in 1991 and (with Salamon) a Steele Prize in 2017.

\section{Advice to Young Women}

Although mathematics is often thought of as a product of the inspiration and brilliance of individuals, it could not exist without a framework that is sustained by the broader community. It is important to find your way in this community. The first step is probably to find some like-minded people (both fellow students and more senior mathematicians) with whom you can do mathematics and feel validated as a mathematician.

\section{Comments on Women's History Month}

Though the situation is much better than it was, women's contributions and potential are still greatly undervalued, both by themselves and by others. This special month provides more visibility for a variety of women with different approaches and contributions to the world of mathematics.

\section{Photo Credit}

Photo of Dusa McDuff courtesy of Dusa McDuff. 


\section{Sophie Morel}

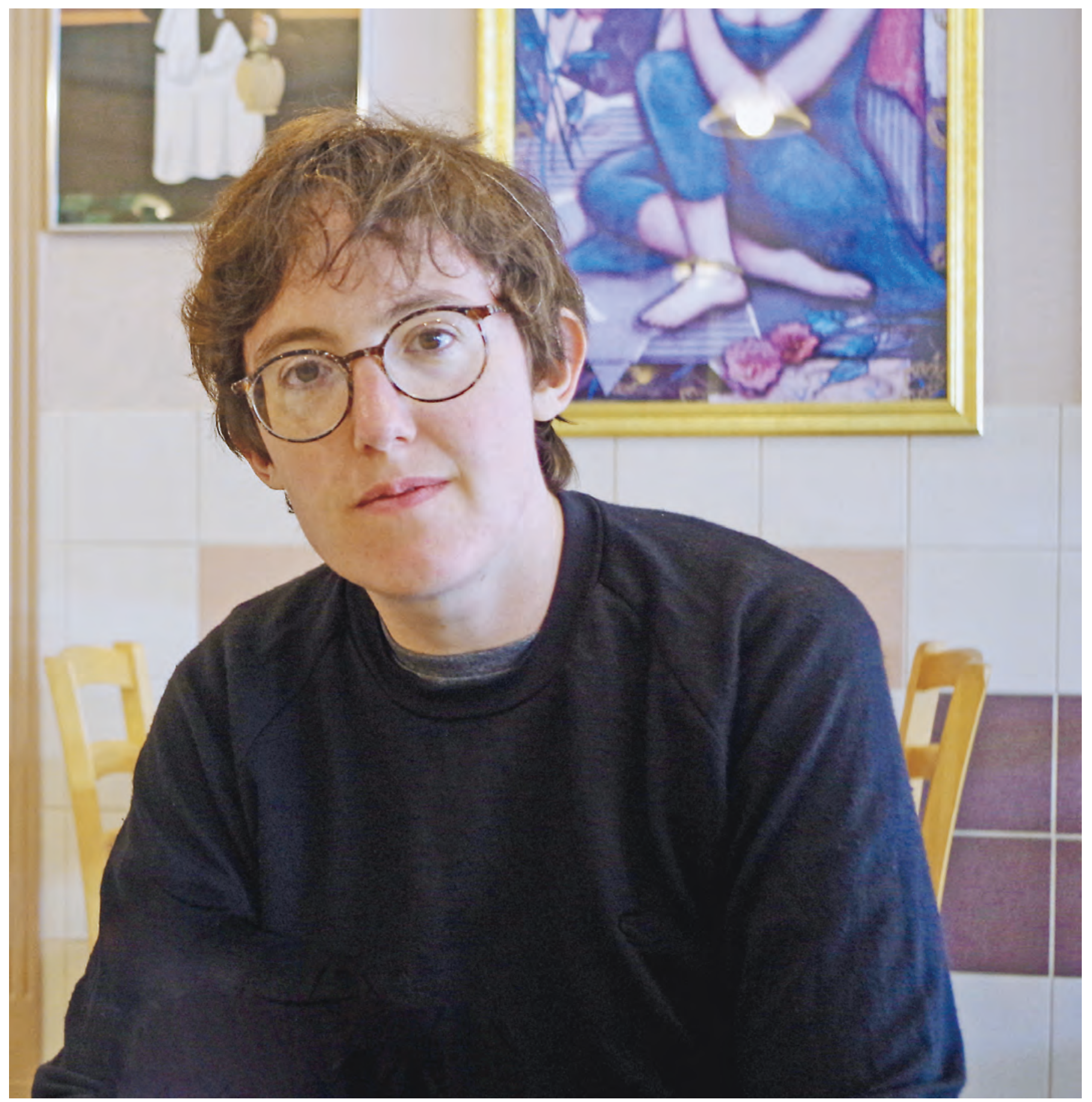




\section{WOMEN'S}

\section{HISTORY}

MONTH

\section{Synopsis of Research}

Sophie Morel's main interest of study is Shimura varieties, more broadly, the arithmetic Langlands program. She is also interested in motives, the geometric Langlands program, and the derived aspects of the Langlands program. She is currently studying automorphic representations appearing in the cohomology of moduli stacks of shtuka, and trying to understand derived deformation rings. Moduli stacks of shtuka, originally due to Drinfeld, play the role of Shimura varieties for the Langlands program over function fields, but without all the restrictions that come with Shimura varieties-so they are much more powerful.

Morel discovered a new way to calculate intersection cohomology and the intersection complex of a stratified scheme, which involves truncations by weights instead of cohomological degree. Here weights refer to Frobenius weights as in the Weil conjectures. (The truncation by cohomological degree formula is due to Deligne.) This way of calculating the intersection complex is an algebraic analogue of a more topological construction due to Goresky, Harder, and MacPherson called "weighted cohomology."

\section{Biography}

Sophie Morel studied at École Normale Supérieure in Paris and defended her PhD in December 2005 at the Université Paris-Sud under the supervision of Gérard Laumon. She was a Clay Research Fellow from 2006 to 2011, and joined the Harvard University mathematics department in 2009 as professor of mathematics. Morel is the first tenured woman in mathematics at Harvard. She joined the Princeton University mathematics department in 2012. She gave an invited talk at the 2010 International Congress of Mathematicians in Hyderabad, India, was awarded one of the prestigious European Mathematical Society Prizes in 2012, and received an Aisenstadt chair at CRM in Montréal in 2015. Morel has been a member of the Institute for Advanced Study in Princeton in 2006-2009, 2010-2011, and 2012-2013.

\section{Advice to Young Women}

My advice for young women is ignore people who try to put you down and just do your thing. Don't feel pressured to represent all female mathematicians. Also, as you go up the mathematical ladder of life, be sure to encourage people who are more junior than you.

\section{Photo Credit}

Photo of Sophie Morel courtesy of Theodore Ehrenborg. 


\section{Andrea Nahmod}

Ceustructions Inveniduce.

bbs nea sure (Bourgain)

$p \leq 6$ fouringor boonsing

defocussing

consturction

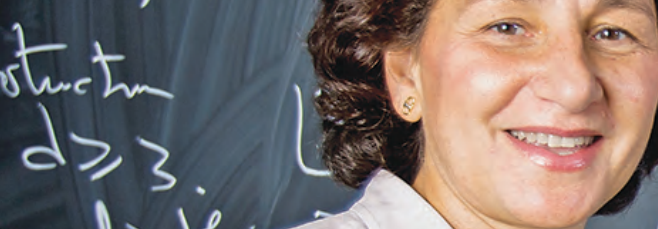

Aow do th.

$i \partial_{t} z+\Delta z=N\left(S(t) u_{0}^{\omega}+z\right)$

Norma

$W_{e}{ }^{\prime}$

Littewod Bley de comp $\rightarrow$ fre untera chin strichartz- dispension

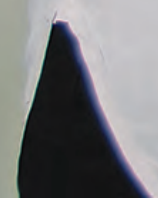




\section{WOMEN'S}

\section{HISTORY}

MONTH

\section{Synopsis of Research}

Andrea Nahmod is professor of mathematics at the University of Massachusetts Amherst. Broadly speaking, her research interests are in two interrelated fields: nonlinear Fourier and harmonic analysis, and the theory of partial differential equations modeling wave propagation phenomena. These are two areas that intimately relate to each other by way of decompositions, frequency interactions analysis, and nonlinear estimates. Nahmod contributed to the understanding of bilinear singular pseudo-differential operators naturally arising in analysis and PDEs and to the wave-packet analysis techniques successfully used in their study. She has also worked on questions aimed at understanding the dynamics of nonlinear waves arising in geometry, ferromagnetism, and gauge field theories.

Over the past ten years Nahmod has been working to analyze and gain a more quantitative understanding of the effect of randomness in the dynamical evolution of PDEs in various regimes, following Bourgain's seminal ideas in the 1990s, integrating tools from probability into her research. There are a myriad of fascinating questions to be explored in exciting and developing new directions at the forefront of nonlinear PDEs. Nahmod likes approaching a problem in new ways, to move the problem forward in a different fashion.

\section{Biography}

My interest in mathematics started at a young age. My fondest memories include sitting in my mother's kitchen as an elementary school child doing my math homework.

My undergraduate thesis advisor and mentor in Argentina was Cora Sadosky. She taught me not only harmonic analysis but also what mathematical research is about. She also helped me to pursue my $\mathrm{PhD}$ in the US.

I received my $\mathrm{PhD}$ degree in mathematics from Yale University under the supervision of Ronald R. Coifman. He taught me a great deal of mathematics, and I am deeply indebted to him for the generosity of his ideas and his contagious enthusiasm and love of mathematics. I have tried to pay these forward with my own students. I also learned a great deal of mathematics from Karen Uhlenbeck whom I was lucky to start collaborating with while I was at UT Austin in the mid-1990s. I have found my stays at the Institute for Advanced Study in Princeton as a member in 1997-1998 and in 2003-2004 to be very fruitful; similarly, my stay as the Sargent-Faull Fellow at Harvard's Radcliffe Institute for Advanced Study in 2009-2010. In 2013-2014 I received a Simons Fellowship and in fall 2015 I was a Simons Professor at MSRI in Berkeley, where I also co-organized a jumbo program. At UMass Amherst, I was named the 2015-2016 Spotlight Scholar and was a recipient of the Award for Outstanding Accomplishments in Research and Creative Activity in 2016. I have been a Fellow of the AMS since 2015.

\section{Advice to Young Women}

If I had to give advice to young women in regards to developing one's own research program, I would say: have a broad mathematical culture, follow your intuition, keep a long view about research, and love what you do.

\section{Comments on Women's History Month}

Despite so much hard-fought progress for equal rights for women by so many before my generation and in my generation, I still imagine a more equitable workplace for women-one where our biases, implicit and otherwise, fade away. Equal pay for equal work is right and it is fair, and it is past time that we as a society demand it and work together to achieve it-all of us, because women are also mothers, sisters, spouses, and daughters, and they all deserve as much respect.

\section{Photo Credit}

Photo of Andrea Nahmod courtesy of John Solem. 


\section{Lillian Pierce}

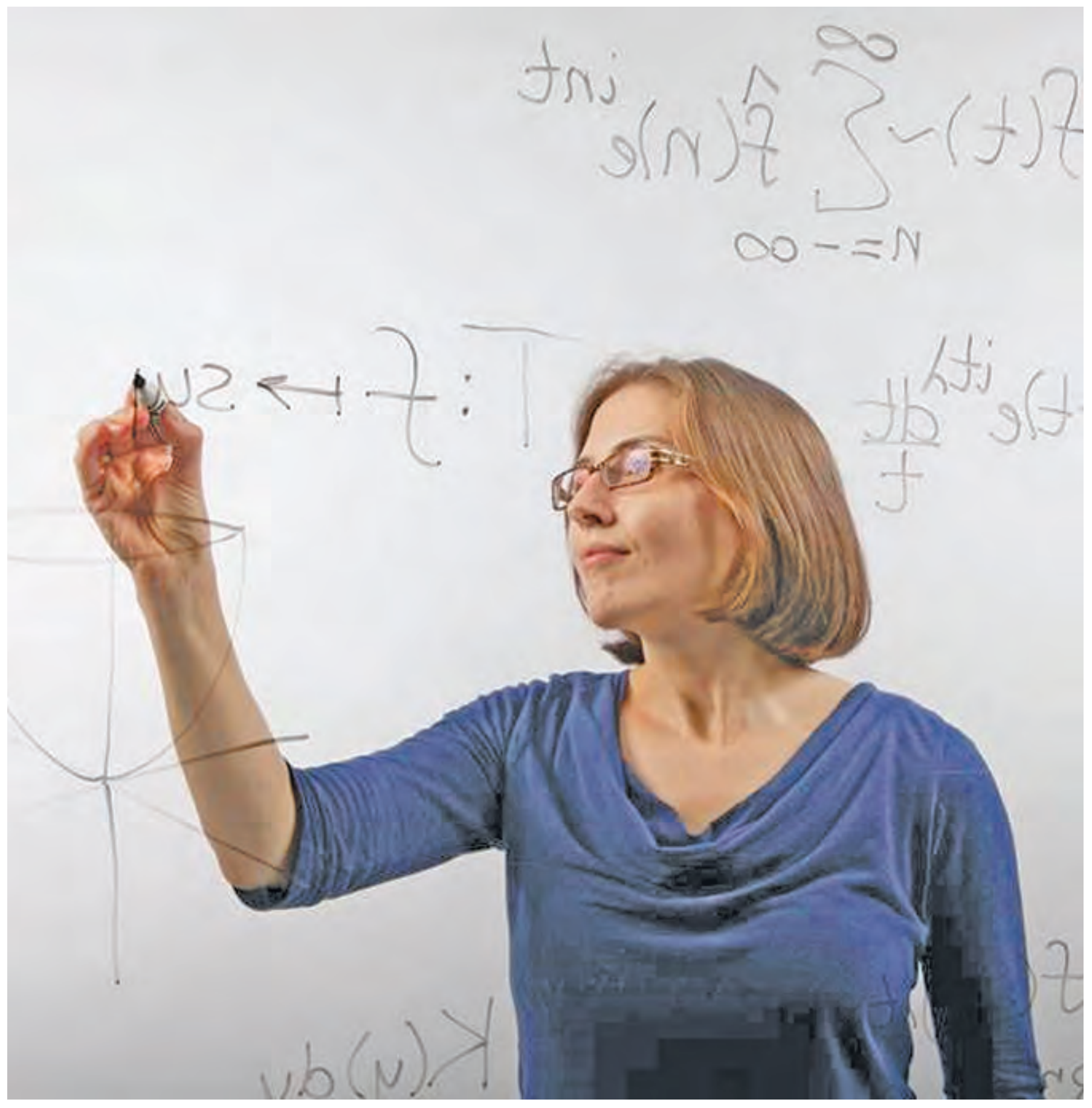




\section{WOMEN'S}

\section{HISTORY}

MONTH

\section{Synopsis of Research}

Lillian Pierce works in the areas of analytic number theory and harmonic analysis. Many of her results have been on $p$-torsion in class groups of number fields, where she has made progress toward conjectured strong upper bounds by developing diverse methods involving sieves, counts for number fields, and zero-density results for automorphic $L$-functions. She has also worked on the circle method, both in the classical context of counting solutions to systems of Diophantine equations and in applications to discrete operators in harmonic analysis. One of her core interests is in bounding character sums and oscillatory integrals, which play a key role in both fields. In particular, her interest in oscillatory integrals has led to work on a new class of Carleson operators that exhibits Radon-type behavior. Pierce's long-term interests are in clarifying and building on deep connections between analysis and number theory.

\section{Biography}

Lillian Pierce grew up in a small town in California and was primarily educated at home, with a significant focus on violin performance. As an undergraduate at Princeton, she completed the pre-medical curriculum but meanwhile majored in math, because its clarity and precision had seemed appealing since childhood. The intensive curriculum and the experience of independent work with encouraging mentors made it clear that a career as an academic mathematician looked like a perfect fit. She then completed graduate work at Oxford and Princeton, followed by postdocs at the IAS and Oxford, and after a year as a Bonn Junior Fellow took up a faculty position at Duke University. Pierce was a Rhodes Scholar and Marie Curie Fellow. She has recently been awarded the AWM-Sadosky Research Prize in Analysis, an NSF CAREER grant, and a von Neumann Fellowship at the Institute for Advanced Study. Having benefited tremendously from the generosity of many mentors, Pierce works consciously to build an inclusive atmosphere for 21 st-century mathematics.

\section{Advice to Young Women}

I find it important to remember what I love about math. In fact, it's very simple. I like to understand things, down to the last detail. There are many other aspects to a career in mathematics, but when I need to, I know to shut all of that out and just return to this basic enjoyment in understanding.

\section{Comments on Women's History Month}

Role models matter. I was shocked when my son, at the age of three, blurted out, "Boys can't do math." Well, he was right in that at that time in our family the girls were the ones talking about math. But it was time to bring him into the club right away. For my own role models, I continually add to a stockpile of photographs and articles about women throughout history who startle me with their determination, insights, goals, and struggles. I know that my mother couldn't have gone to the undergraduate institution I attended because it didn't accept women at that time. Yet when I showed up, I was welcomed with open arms, gained a terrific education, and graduated as valedictorian of my class. In the next generation's turn, I hope we will make new, wonderful, flexible opportunities available for all our children.

\section{Photo Credit}

Photo of Lillian Pierce courtesy of Duke Photography. 
Jill C. Pipher

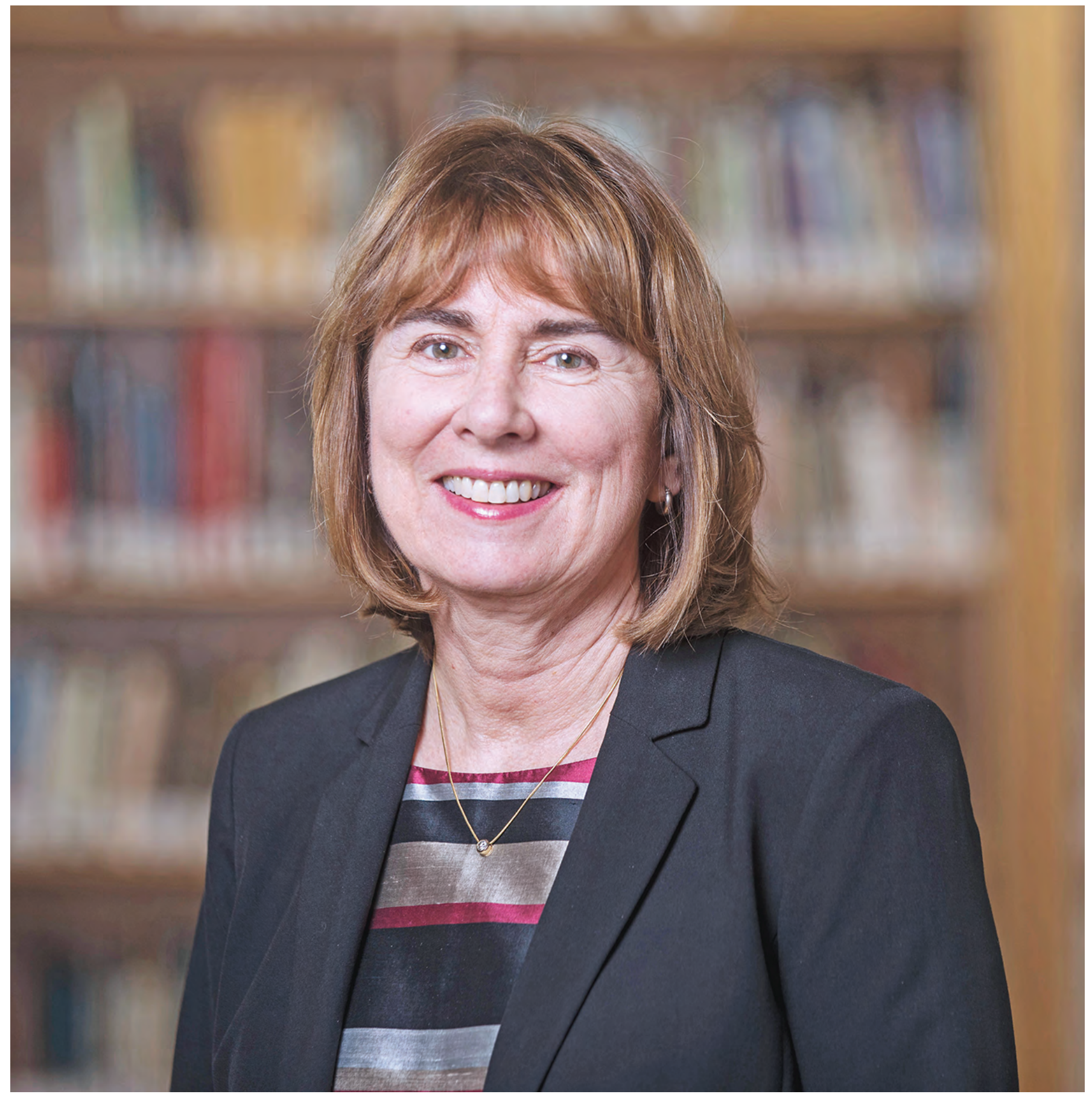




\section{WOMEN'S}

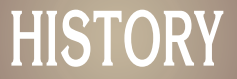

MONTH

\section{Synopsis of Research}

Jill C. Pipher's primary areas of research are harmonic analysis and non-smooth linear elliptic and parabolic boundary value problems. She has also worked on lattice-based cryptography, particularly the NTRU public key encryption system and related algorithms. Her work in harmonic analysis has focused on singular integral operators, the theory of weights, and especially the associated multi-parameter theory. In elliptic and parabolic PDEs, she has contributed to key developments in the theory of divergence form equations with non-smooth coefficients, of perturbations of operators, of higher order operators like the bi-harmonic equation, and of non-symmetric equations, where she has helped to create new tools for solving boundary value problems that yield precise connections between the smoothness of the coefficients and the regularity of the solutions.

\section{Biography}

Jill C. Pipher is vice president for research at Brown University and Elisha Benjamin Andrews Professor of Mathematics. In addition to her administrative responsibilities, she is currently co-teaching a freshman class in mathematics, advising graduate students, and serving on committees of several professional societies. She was the founding director of the Institute for Computational and Experimental Research in Mathematics (ICERM). She received her PhD from UCLA in 1985, spent five years at the University of Chicago, and came to Brown in 1990 as associate professor. She was a co-founder of NTRU Cryptosystems, Inc., now part of Security Innovation, Inc., and jointly holds four patents for NTRU algorithms. She has been awarded an NSF Postdoctoral Fellowship, an NSF Presidential Young Investigator Award, and an Alfred P. Sloan Foundation Fellowship, and she is a Fellow of the American Mathematical Society. She served as president of the Association for Women in Mathematics from 2011-2013, and was a National Women's History Month 2013 Honoree. In 2015, she was elected to the American Academy of Arts and Sciences. She is now president-elect of the AMS.

\section{Advice to Young Women}

First, I think it is important to be flexible and open-minded about the variety of mathematical careers available. There are many types of jobs within academia, and many more without. I found that being open to different possibilities at critical career junctions in my life had a very positive effect on my sense of control over my future, boosting my confidence as well. Second, I strongly advise seeking, or even creating, opportunities to build professional networks and find mentorship. This is important for everyone at an early stage in their career, but especially for women and other members of under-represented groups.

\section{Comments on Women's History Month}

In 2013, I was honored to participate in a panel of distinguished Rhode Island women to discuss the importance of STEM education and skills, part of a celebration of National Women's History Month hosted by Senator Jack Reed. Every year, Senator Reed teams up with the Women's Fund of RI to celebrate the accomplishments and contributions of women. The 2013 theme was "Women Inspiring Innovation Through Imagination: Celebrating Women in Science, Technology, Engineering, and Mathematics." Like other panel discussions that bring together women in STEM fields, the discussion was honest, sometimes moving, and always fascinating. I am grateful that Women's History Month is a catalyst for these important events that help us reflect on where we've been, where we are now, and how we can effect change in the future.

\section{Photo Credit}

Photo of Jill C. Pipher courtesy of Brown University. 
Emily Rieh|

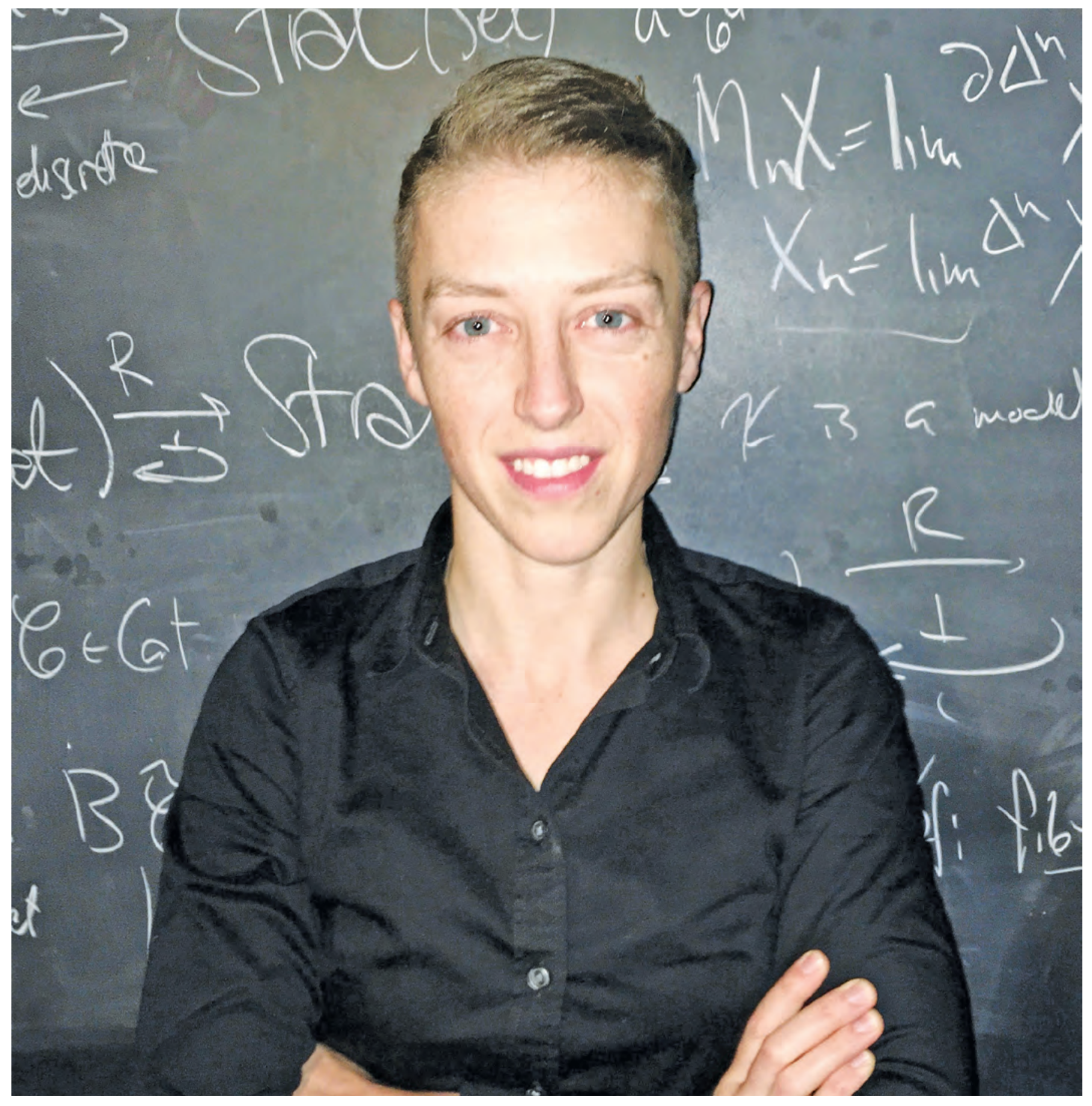


WOMEN'S

HISTORY

MONTH

\section{Synopsis of Research}

A category provides a template for a mathematical theory supplying nouns, to represent those mathematical objects, and verbs, to represent the transformations between them. But as the objects mathematicians study increase in complexity, a more sophisticated linguistic palate is required, including adjectives, adverbs, conjunctions, and so forth. Such objects live most naturally in weak infinite-dimensional categories, commonly nicknamed $\infty$-categories. Pioneering work of André Joyal and Jacob Lurie to extend ordinary 1-category theory to $\infty$-categories is "analytic" in the sense that it is developed using the combinatorics of a particularly convenient model of a weak infinite-dimensional category. Emily Riehl's joint project with Dominic Verity redevelops this theory "synthetically," with proofs that hold in any $\infty$-cosmos, their axiomatization of the "universe" in which $\infty$-categories live as objects. Their $\infty$-cosmological approach both simplifies definitions and proofs of certain key components from the analytic theory and also generalizes them without change to other well-behaved models of infinite-dimensional categories. In future work, Riehl plans to write a textbook exposing these developments, originally described in a series of seven research papers, and also plans to apply similar techniques to develop the theory of higher-dimensional analogues of $\infty$-categories.

\section{Biography}

I specialize in category theory, particularly as related to homotopy theory, and have been assistant professor of mathematics at Johns Hopkins University since 2015. Prior to this, I was an undergraduate at Harvard University, completed Part III of the Maths Tripos at Cambridge, earned my $\mathrm{PhD}$ in 2011 from the University of Chicago, and then spent four years at Harvard as an NSF and Benjamin Peirce Postdoctoral Fellow. I am the author of two books, Categorical Homotopy Theory (Cambridge 2014) and Category Theory in Context (Dover 2016), both of which are freely available online; a co-author of 20 published research articles; and have written many other expository works with various degrees of polish. I am the founder of the Kan Extension Seminar, an online graduate reading course in category theory with participating students from around the globe, and a co-host of The n-Category Café. I serve as an editor for Journal of Homotopy and Related Structures; Homology, Homotopy, and Applications; and Cahiers de Topologie et Géométrie Différentielle Catégoriques. I am a member of the AMS Mathematics Research Community Advisory Board and chair of the AMS Web Editorial Group. I have been awarded an NSF grant and a CAREER award to support my work. I have also been recognized for excellence in teaching at both Johns Hopkins and at Harvard. I am currently advising somewhere between two and four $\mathrm{PhD}$ students and mentoring one postdoctoral fellow, and I will be co-organizing an MSRI semester on Higher Categories and Categorification, which will take place in 2020.

\section{Advice to Young Women}

Regarding advice, I would have appreciated being reassured that it's ultimately up to me how I want to spend my time. I particularly enjoy giving talks and am unusually enthusiastic about expository writing, so I say yes to most speaking engagements and take on a lot of extracurricular writing projects. This means less time for research and other things, but it also makes me happier, so feels like a win overall. I think the fetishization of research above all else is really damaging to both the self-confidence of members of our community and to the community as a whole, which requires superlative advisors, inspiring teachers, diligent referees, compassionate mentors, efficient organizers, and so on to function in a healthy way.

Photo Credit

Photo of Emily Riehl courtesy of Mona Merling. 
Karen E. Smith

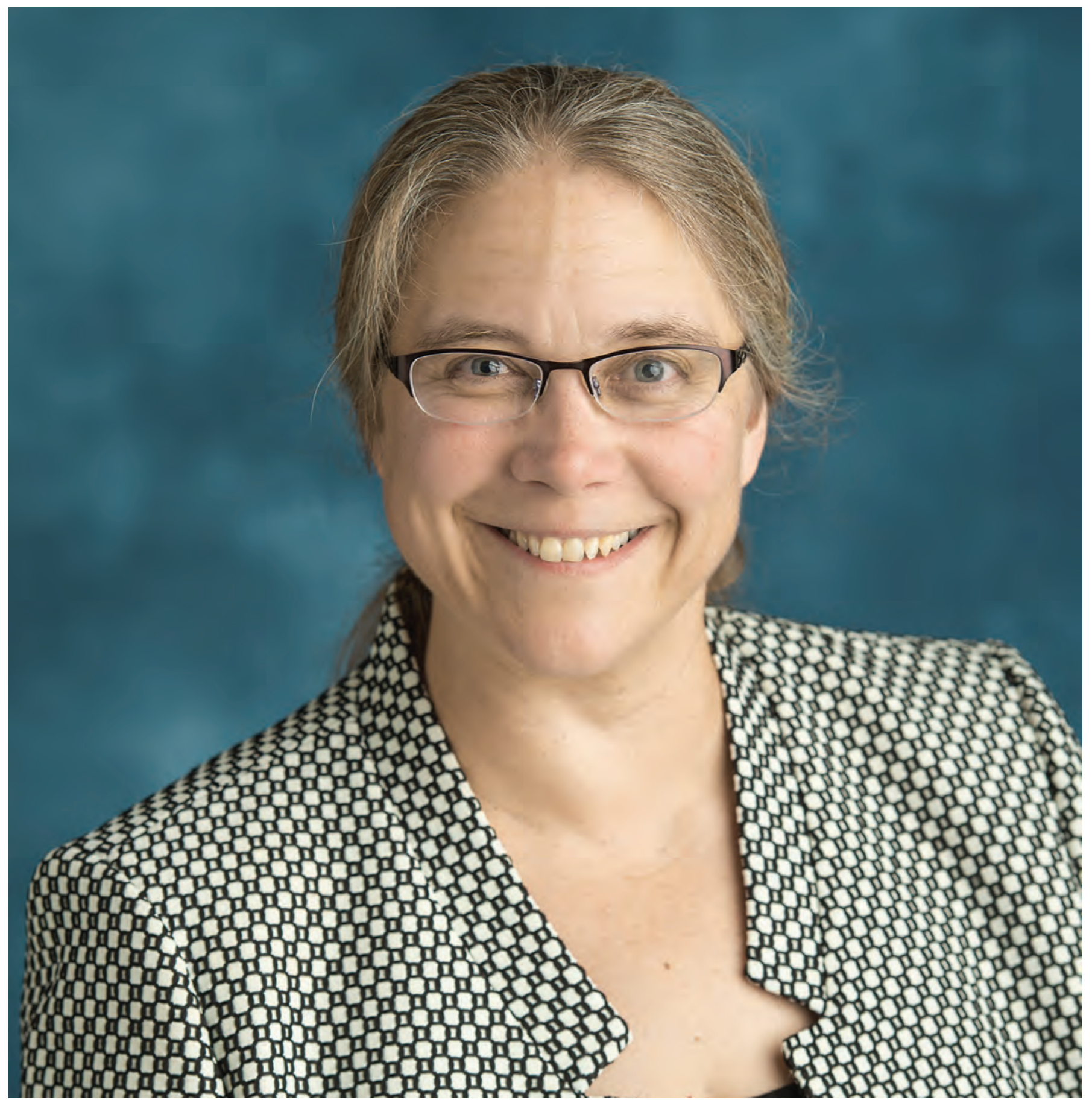


WOMEN'S

HISTORY

MONTH

\section{Synopsis of Research}

Karen E. Smith's research is in commutative algebra and algebraic geometry. She is known for using prime characteristic techniques in commutative algebra to solve problems in algebraic geometry and other subjects. For example, she has been a pioneer in understanding the singularities of varieties over finite fields, characterizing them by considering the Frobenius action on local cohomology modules. Her paper linking the multiplier ideal with the test ideal has been at the center of an industry connecting the singularities and tools of the minimal model program with singularities and tools in prime characteristic commutative algebra. With Robert Lazarsfeld and Lawrence Ein, Smith discovered a surprisingly tight relationship between symbolic and ordinary powers of ideal sheaves in smooth complex varieties, founding a major field of investigation. Jointly with Michel Van den Bergh, she developed a theory of differential operators in prime characteristic for strongly F-regular rings that spawned new singularity invariants such as the F-signature. She is especially proud of her success training over sixteen $\mathrm{PhD}$ students and about as many post-docs, many of whom are now powerful researchers in their own right.

\section{Biography}

Karen E. Smith was born and raised near the Jersey shore and never heard of graduate school until after graduating college. She was one of a small cohort of math majors at Princeton, and, thinking she was no good at math, accepted a high school teaching job upon graduation. While she loved high school teaching, she found herself discouraged by an extremely difficult job. Luckily, she ran into a former peer from Princeton who mentioned he was a $\mathrm{PhD}$ student in mathematics, and, in fact, getting paid to teach calculus to college freshman while deferring his student loans. That was enough for her to decide to apply to graduate school. She enjoyed her job as a Graduate Student Instructor at the University of Michigan, where she was surprised to discover she was quite well prepared for her graduate courses. She finished her $\mathrm{PhD}$ in commutative algebra with Mel Hochster in 1993, and accepted an NSF post-doctoral fellowship at Purdue University under the direction of Craig Huneke. As a post-doc, she began studying algebraic geometry, a process that accelerated when she moved to Boston to accept a Moore Instructorship at MIT. In Boston, she found many math friends and peer collaborators, laying the foundations of a successful research program aimed at using the Frobenius map in characteristic $p$ to prove theorems about singularities and vanishing of cohomology. Her work was recognized with the Satter Prize in 2001.

In 1997, Smith moved back to Ann Arbor with her husband Juha Heinonen, where they both accepted professorships. As a young professor, she birthed her first child, Sanelma, who travelled to many conferences as an infant, often with dad or grandma as babysitter. Indeed, because
Juha was so supportive and she had accumulated so many great ideas in Boston, the years after Sanelma was born were quite productive mathematically, even with a small child. Five years later, she accomplished what she still considers her most impressive feat: birthing twins Tapio and Helena and completing an amazing family.

Smith faced a major setback in 2007, when her husband Juha was taken by cancer. Her youngest were only three years old when he fell ill, so she has not been able to travel or work as much since then. Fortunately, the vibrant mathematical community in Ann Arbor has enabled her to continue her mathematical work. She loves working with post-docs and students, and loves teaching and mentoring at all levels.

She is currently associate chair of graduate studies at the University of Michigan and the faculty advisor for the local student chapter of AWM. She is also an important mentor for many of Michigan's African American math majors and has a special interest in helping and mentoring the growing number of undergraduates who transfer to UM from community colleges. Now remarried to another Finnish analyst, Kai Rajala, she is enjoying three teenagers at home and countless twenty-something students in her office, while trying to prove new theorems about rings of differential operators in characteristic $p$.

\section{Advice to Young Women}

Start where you are at; do not compare yourself with others. Learn the basics well- "exposure" to fancy stuff is fine too, as long as you also take the time to deeply understand the basics. Remember to have fun, with math and with life. Find places you thrive, mathematically and otherwise. Be bold seeking advice, but remember that not all advice is good. Above all else, marry a feminist.

\section{Comments on Women's History Month}

I still remember the thrill when, as a sophomore in Math 325-Professor Moore's abstract algebra course at Princeton-I was reading about the founder of modern algebra in Jacobson's textbook and saw the pronoun she. Emmy Noether. Probably until that point I would have said that I never felt constrained by gender, but wow. Everything was different after that in a way that is hard to explain to those privileged enough to never, consciously or unconsciously, question whether or not they belong. While it was a terrific feeling, I wish that tomorrow's daughters would be spared it. Maybe the Notices celebration of Women's History Month can play a tiny role in helping today's daughters see that they really do belong and, in fact, are needed.

Photo Credit

Photo of Karen E. Smith (c) Eric Bronson, Michigan Photography. 


\section{Gigliola Staffilani}

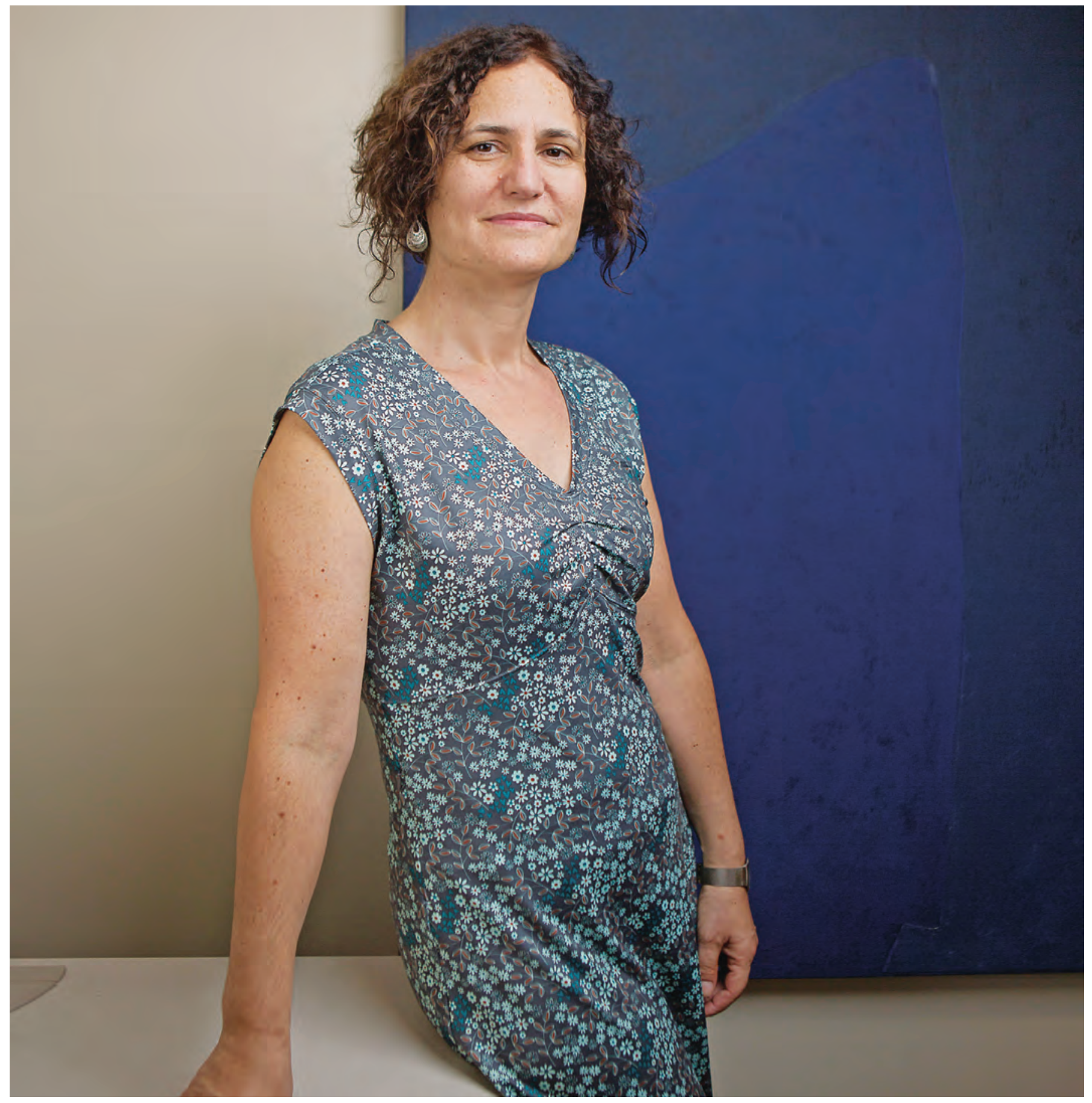


WOMEN'S

HISTORY

MONTH

\section{Synopsis of Research}

Gigliola Staffilani is interested in studying certain partial differential equations that model nonlinear wave phenomena. Often, because of nonlinearity of the problem, explicit solutions cannot be found. Hence her job is to be able to deduce certain properties of these solutions without knowing how they look. These properties include existence, uniqueness and stability of the solutions themselves, their long-time dynamics (including asymptotics), and their interactions. Staffilani has been enjoying this line of research for many reasons, but most of all because the tools used to attack the relevant questions come from different areas of mathematics: harmonic and Fourier analysis, analytic number theory, geometry, probability, and dynamical systems. In particular, in the last five years Staffilani has been introducing more and more probabilistic concepts in the work that she has done. Often it is not possible to say that every initial profile generates a wave solution, but we would like to prove it generically. Probability helps us in defining with rigor what "generically" means. The most enjoyable part of Staffilani's research career so far has been walking the path of investigation together with her collaborators, starting with her advisor Carlos Kenig and the I-team (Jim Colliander, Markus Keel, Hideo Takaoka, and Terry Tao) in the early years of her career. From her collaborators Staffilani learned an incredible amount of mathematics, and she shared with them several moments of frustration as well as of enthusiasm.

\section{Biography}

Gigliola Staffilani is the Abby Rockefeller Mauzé Professor of Mathematics at MIT. She received MS and PhD degrees from the University of Chicago in 1991 and 1995. Following a Szegö Assistant Professorship at Stanford, she had faculty appointments at Stanford, Princeton, and Brown before joining the MIT mathematics faculty in 2002. At Stanford, she received the Harold M. Bacon Memorial Teaching Award in 1997, and was given the Frederick E. Terman Award for young faculty in 1998. She was a Sloan fellow from 2000-2002. At MIT Staffilani served as co-chair of the Graduate Student Committee in Pure Mathematics from 2009-2013, and since 2015 has been the Faculty Diversity Officer. In 2013 she was elected member of the Massachusetts Academy of Science and a Fellow of the AMS, and in 2014 Fellow of the American Academy of Arts and Sciences. In 2017 she received a 2017 Guggenheim Fellowship and a 2017 Simons Fellowship in Mathematics. As a member of the department's edX group, Staffilani received the inaugural MITx Prize for Teaching and Learning in MOOCs by the MIT Office of Digital Learning. Gigliola has had six graduate students, including three current ones. She has also advised dozens of math majors at MIT.

\section{Advice to Young Women}

I recommend to anybody who wants to pursue research in mathematics to work with other mathematicians. The model of the lonely researcher in his ivory tower does not match with most of the mathematicians I know. This is a myth that definitely needs to be busted: it is dangerous and not encouraging.

\section{Comments on Women's History Month}

It is very important to remind people that there are still too few women in mathematics. Great efforts have been made in recent years to improve this number, but a lot more work has to be done. I hope that celebrating Women's History Month will at the same time recognize the achievements of many women mathematicians while inspiring young ones to dedicate their intellectual power to prove more new and powerful theorems.

\section{Photo Credit}

Photo of Gigliola Staffilani by Bryce Vickmark. 
Éva Tardos

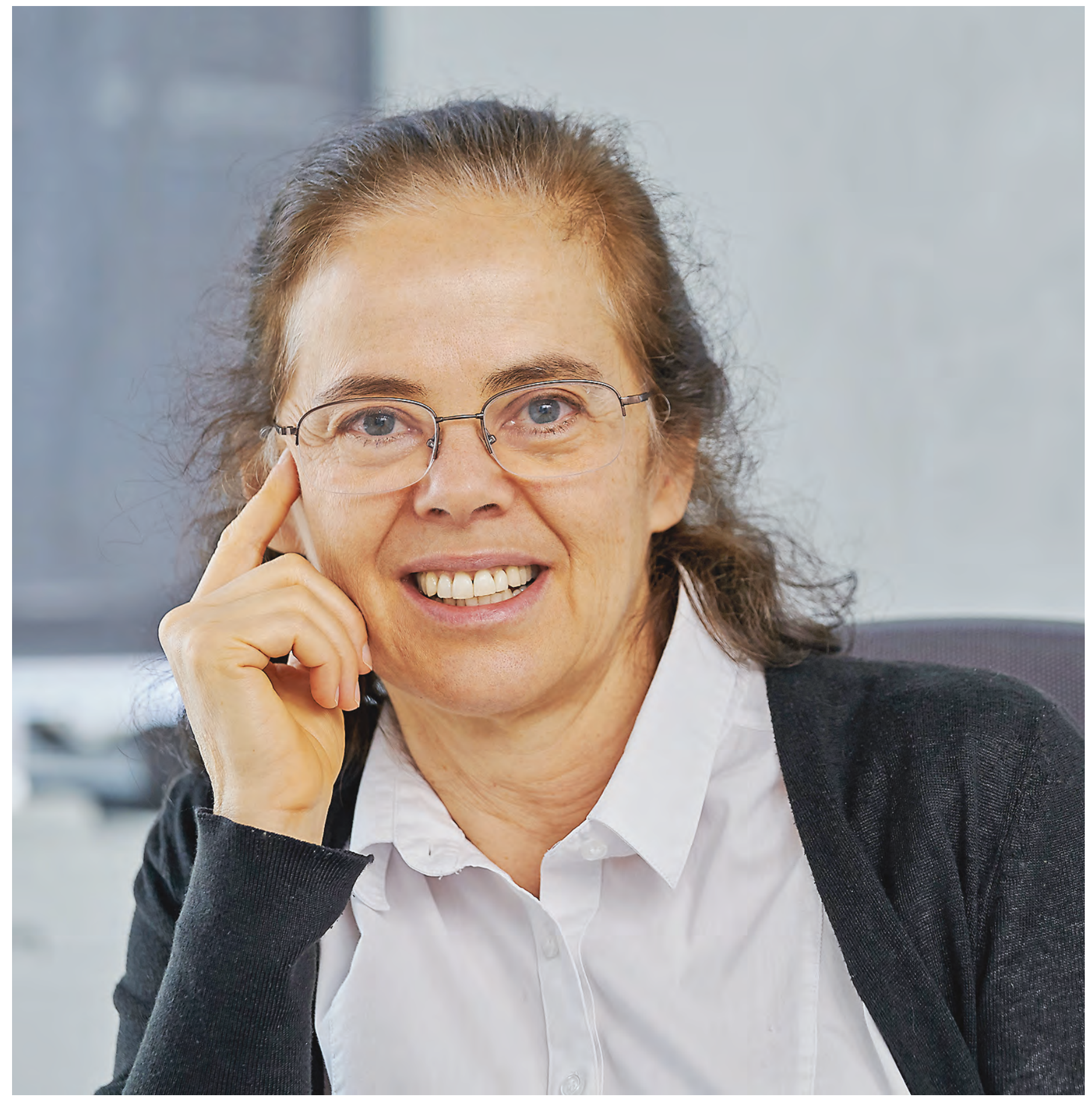


WOMEN'S

HISTORY

MONTH

\section{Synopsis of Research}

Éva Tardos's research interest is algorithms and algorithmic game theory. She is most known for her work on network-flow algorithms and quantifying the efficiency of selfish routing. For network-flow algorithms, she developed an efficient algorithm to find minimum-cost flow in a network in time that depends only on the size of the networks, independent of the magnitude of the costs. She also extended this result to a class of combinatorial linear programs. Extending it to all linear programming remains an important open problem in the field. For the last fifteen years her work has been focused at the interface of economics and the theory of computing, understanding how to design systems and algorithms for selfish users. An important example is traffic routing, car traffic or Internet traffic. Drivers or routers choose the path of the traffic to minimize delay, where the time to traverse an edge increases with congestion. The classical Braess paradox shows that this individual optimization may not lead to an overall optimal solution: deleting edges in the network can improve travel time for all users. Tardos's results offer bounds quantifying the resulting inefficiency. Similar analysis also offers insight on the quality of simple auction systems, such as eBay or advertisement auctions used by many platforms. In most of these applications both the system and the individual participants use machine learning to optimize the outcome. Tardos is currently extending her work to identify and understand good learning algorithms in such situations.

\section{Biography}

Éva Tardos is Jacob Gould Schurman Professor of Computer Science at Cornell University. She received her BA and $\mathrm{PhD}$ from Eötvös University in Budapest. She joined the faculty at Cornell in 1989 after a sequence of postdocs including time at Berkeley and MIT. At Cornell, she was computer science department chair from 2006-2010. She has been faculty advisor for the student group Women in Computing at Cornell since its founding, and is serving as the college's diversity lead. She has been elected to the National Academy of Engineering, the National Academy of Sciences, and the American Academy of Arts and Sciences. She is an external member of the Hungarian Academy of Sciences. She is the recipient of a number of fellowships and awards, including the Packard Fellowship, the Gödel Prize, the Dantzig Prize, the Fulkerson Prize, the ETACS prize, and the IEEE Technical Achievement Award. She was selected as the faculty of the year twice by computer science undergraduates. She is editor-in-chief of the Journal of the Association for Computing and has also served as editor of several other journals, including the SIAM Journal of Computing and Combinatorics. She was program committee chair for the 1996 ACM-SIAM Symposium on Discrete Algorithms, the 2005 IEEE Symposium on Foundations of Computer Science, and the 2013 ACM Conference on Economics and Computation.

\section{Advice to Young Women}

I have greatly enjoyed working on a wide range of problems during my career and would encourage young researchers to be open to exciting new opportunities in research, whenever they have the right basic tools to contribute.

\section{Comments on Women's History Month}

I'd like to recommend the one-minute GE YouTube video, "What If Millie Dresselhaus, Female Scientist, was Treated Like A Celebrity." 1

\section{Photo Credit}

Photo of Éva Tardos by Dave Burbank. 


\section{Chelsea Walton}

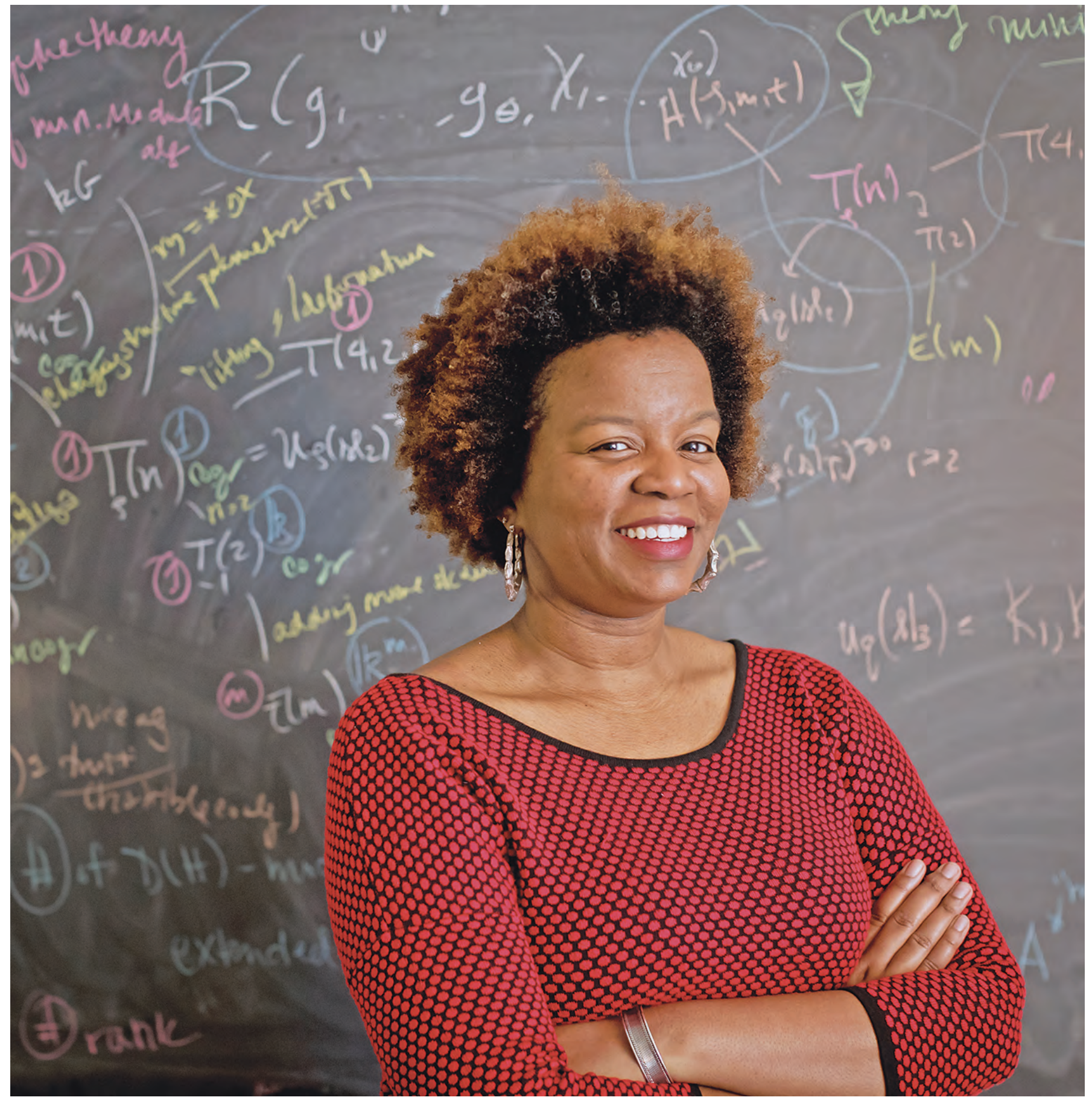




\section{WOMEN'S}

\section{HISTORY}

MONTH

\section{Synopsis of Research}

Chelsea Walton is assistant professor of mathematics at Temple University and will start a tenured position at the University of Illinois UrbanaChampaign in fall 2018. Walton's areas of research lie in noncommutative algebra, noncommutative algebraic geometry, quantum algebra, and representation theory. One of her main interests is (co)actions of Hopf algebras and quantum groups.

\section{Biography}

I was born and raised in Detroit, and I'm very proud of being from there. I attended Detroit public schools for my K-12 education, save a couple of years in elementary school. I was super lucky to be able to attend a middle school that had a STEM-oriented curriculum; this planted the seeds in me that doing math for a living would be my dream job. After high school, I enrolled at Michigan State University and University of Michigan for undergrad and graduate school, respectively. My first positions were as an NSF postdoc at University of Washington Seattle and as a Moore instructor at MIT.

If I had to name key lessons that I learned at each stage of my training and career, all would involve communicating math-writing, speaking, collaborating-more effectively. This is actually my favorite part of the job-sharing math. In fact, one way I decide if I'm going to work on a particular project is by imagining myself giving a talk (or, in my mind,"putting on a show") on the subject.

\section{Advice to Young Women}

I was asked to give some advice for young women in math here, but I find that it's tough to provide one-size-fits-all words of encouragement. People have very different needs. So, I highly recommend finding, valuing, and supporting your network of people, in math or not, who can selflessly give you words of encouragement. People who make you a better You. Pick your advisors, mentors, friends, and network carefully, as they will either inspire you or drain you. Choose the inspirers whenever you can, because the happier you are, the more math you will do!

\section{Comments on Women's History Month}

Thank you for including me, and I look forward to reading the other bios of current women in math in this issue and also in future issues of Notices. I'm honored and humbled to be a part of a group of women who could serve as role models for future generations of women and other groups of underrepresented mathematicians.

\section{Photo Credit}

Photo of Chelsea Walton courtesy of Ryan S. Brandenberg/Temple University. 


\section{Amie Wilkinson}

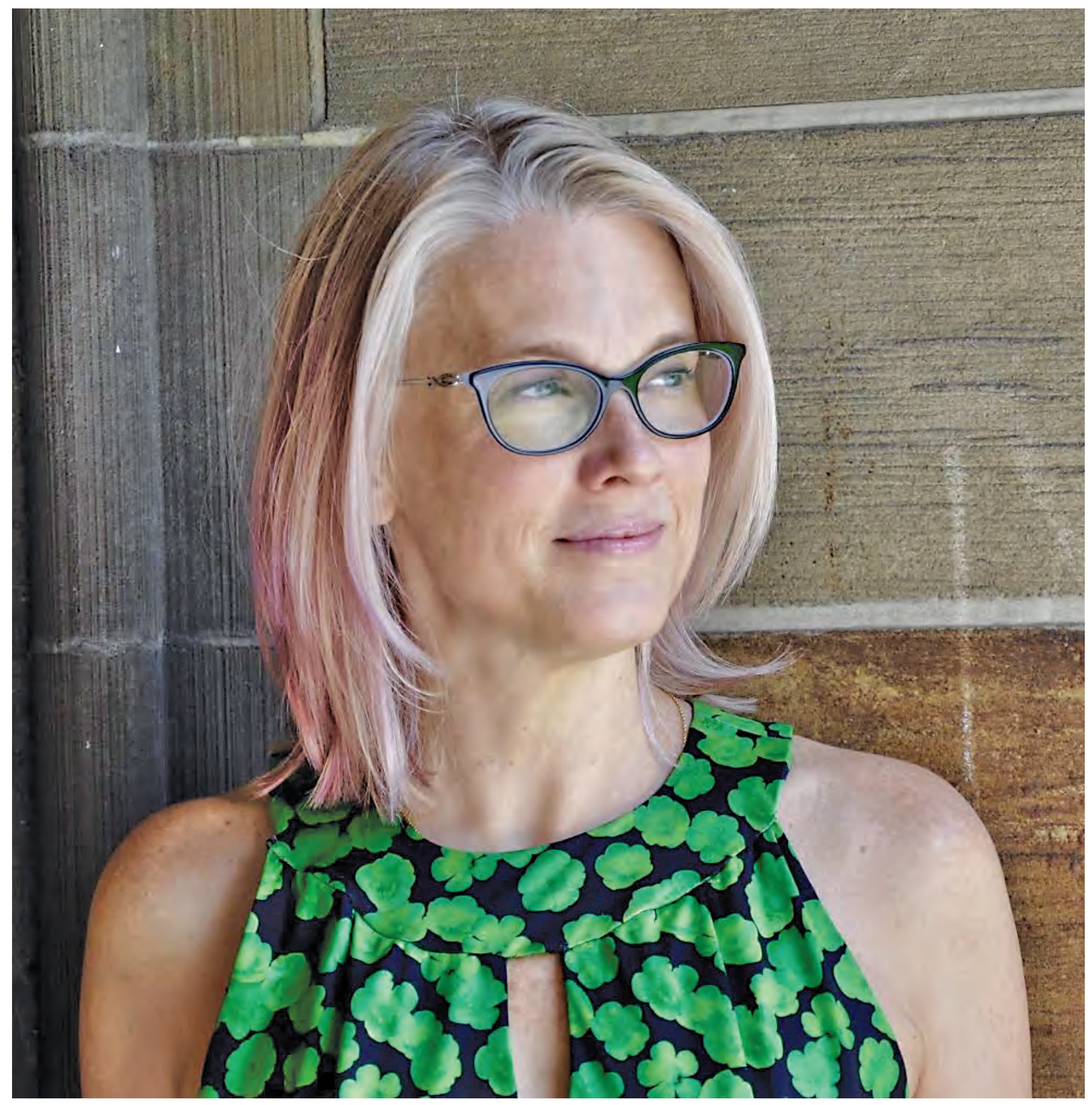




\section{WOMEN'S}

\section{HISTORY}

MONTH

\section{Synopsis of Research}

Amie Wilkinson studies dynamical systems-spaces in motion. Dynamics as a field was born in the late 19th century and grew out of attempts to describe the evolution of physical systems over time, both on large time scales (the stability of planetary motion) and tiny ones (the mixing of particles in an ideal gas). Wilkinson's research is concerned with the interplay between dynamical phenomena and other structures in pure mathematics-geometric, statistical, topological, and algebraic. Her contributions include establishing a general mechanism for stability of mixing (with Keith Burns); proving the $C^{1}$ version of a question of Smale: the generic diffeomorphism has no symmetries (with Christian Bonatti and Sylvain Crovisier); showing that the Weil-Petersson flows arising in Teichmüller theory are mixing (with Burns and Howard Masur); and establishing a version of Boltzmann's ergodic hypothesis for $C^{1}$ diffeomorphisms with positive entropy (with Avila and Crovisier).

\section{Biography}

I have always liked math, and I grew up in ideal circumstances to develop my interest, with supportive family and teachers. Off to an easy start, I still struggled along the way, nearly abandoning in college my plan to become a mathematician. This was due in part to a lack of female role models, but also due to the realization that I was far from the best math student at Harvard. Taking a year off after college to work, I stopped looking at other people and took a long, hard look at math itself. I missed it. I applied to graduate school and chose Berkeley, where I was joined by 100 other people in my class. That suited me fine.

In grad school, I discovered the power of math as a social activity. How, rather than competing with my classmates, I could work with them to understand and discover new things. Mathematics took shape as a massively intricate monument to human creative activity. I had found my place.

Since then, both my confidence and my power as a mathematician have grown. My work has been honored occasionally, for example in an invitation to speak at the ICM, and in being awarded the Satter Prize. With age has come freedom to speak my mind, to act stupid, and to take risks. While research is as interesting as ever, my focus recently has also been on advising and mentoring, on helping to build the dynamics group at the University of Chicago, and on communicating mathematics to the world outside.

\section{Advice to Young Women}

To me, the joy in mathematics lies in the process of discovery, and the realm of dynamics presents an infinite zoo of possibilities to explore. When I was starting out, my goal was to figure out which way was up and how not to get lost. Over time this goal has evolved, and I am driven to discover new species and surprising behaviors in this zoo.

My advice is to try to be patient and enjoy the scenery.

\section{Photo Credit}

Photo of Amie Wilkinson courtesy of Amie Wilkinson. 


\section{Lauren Williams}

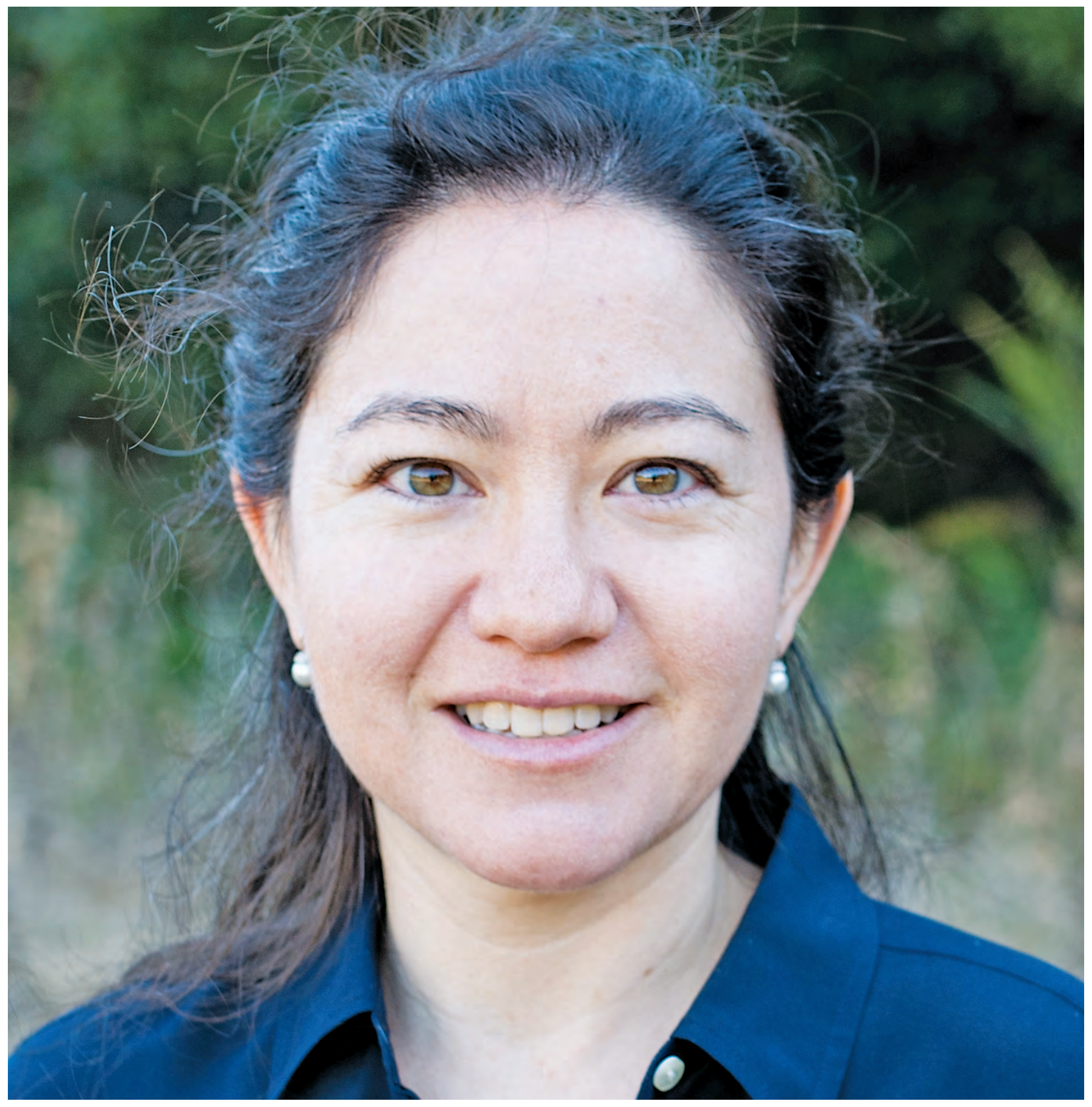




\section{WOMEN'S}

\section{HISTORY}

MONTH

\section{Synopsis of Research}

Lauren Williams is an algebraic combinatorialist, but she particularly enjoys working on problems that are at the interface between algebraic combinatorics and other fields. Some of her favorite research contributions include: a combinatorial formula for the stationary distribution of the asymmetric simple exclusion process (commonly cited as a model for traffic flow and translation in protein synthesis), joint with Sylvie Corteel; a polytopal manifestation of mirror symmetry for Grassmannians, joint with Konstanze Rietsch; a proof of Da Silva's conjecture on realizability of positively oriented matroids, joint with Federico Ardila and Felipe Rincon; the illumination of the role of the positive Grassmannian in the behavior of soliton solutions of the KP equation (used to model shallow water waves), joint with Yuji Kodama.

\section{Biography}

I grew up in a suburb of Los Angeles, the oldest of four girls. I liked math from an early age, but I also loved art, reading, writing poetry, and playing the violin. Once I started participating in math competitions and summer programs, I noticed that there were not very many girls involved; but the fact that my younger sisters were also good at math helped, I think, to mitigate any feelings of isolation. I majored in math at Harvard, spent a year at Cambridge doing Part III of the Mathematical Tripos, and then obtained my PhD from MIT under the supervision of Richard Stanley. After postdoctoral positions at UC Berkeley and Harvard, I joined the faculty at UC Berkeley in 2009, obtaining tenure in 2013. I have two kids, ages two and five, who bring me a lot of joy but also make the task of balancing my various responsibilities much harder! I have graduated six $\mathrm{PhD}$ students so far with five more current students. Some recent awards include the AWMMicrosoft Research Prize, the Rose Hills Innovator Award, a Simons Fellowship, an NSF CAREER Award, a Sloan Fellowship, the Prytanean Faculty Award (a mentoring award for Berkeley faculty), a Distinguished Undergraduate Teaching Award, and the 2018 Hardy Lectureship.

\section{Advice to Young Women}

My advice for young people, both women and men, is to find a subject area and problems that you care deeply about; I think it would be difficult to do good research (or find satisfaction in your work) otherwise. Additionally, mathematical research requires a great deal of patience, as there may be long periods of time when you don't make visible progress. In order to deal with this, I think it's quite important to have a deep reserve of self-confidence. I don't have any magic recipe for how to find this confidence, but I think it helps to surround yourself as much as possible with people who support you and to try not to compare yourself too much with others.

\section{Photo Credit}

Photo of Lauren Williams courtesy of Lauren Williams. 


\section{Melanie Matchett Wood}

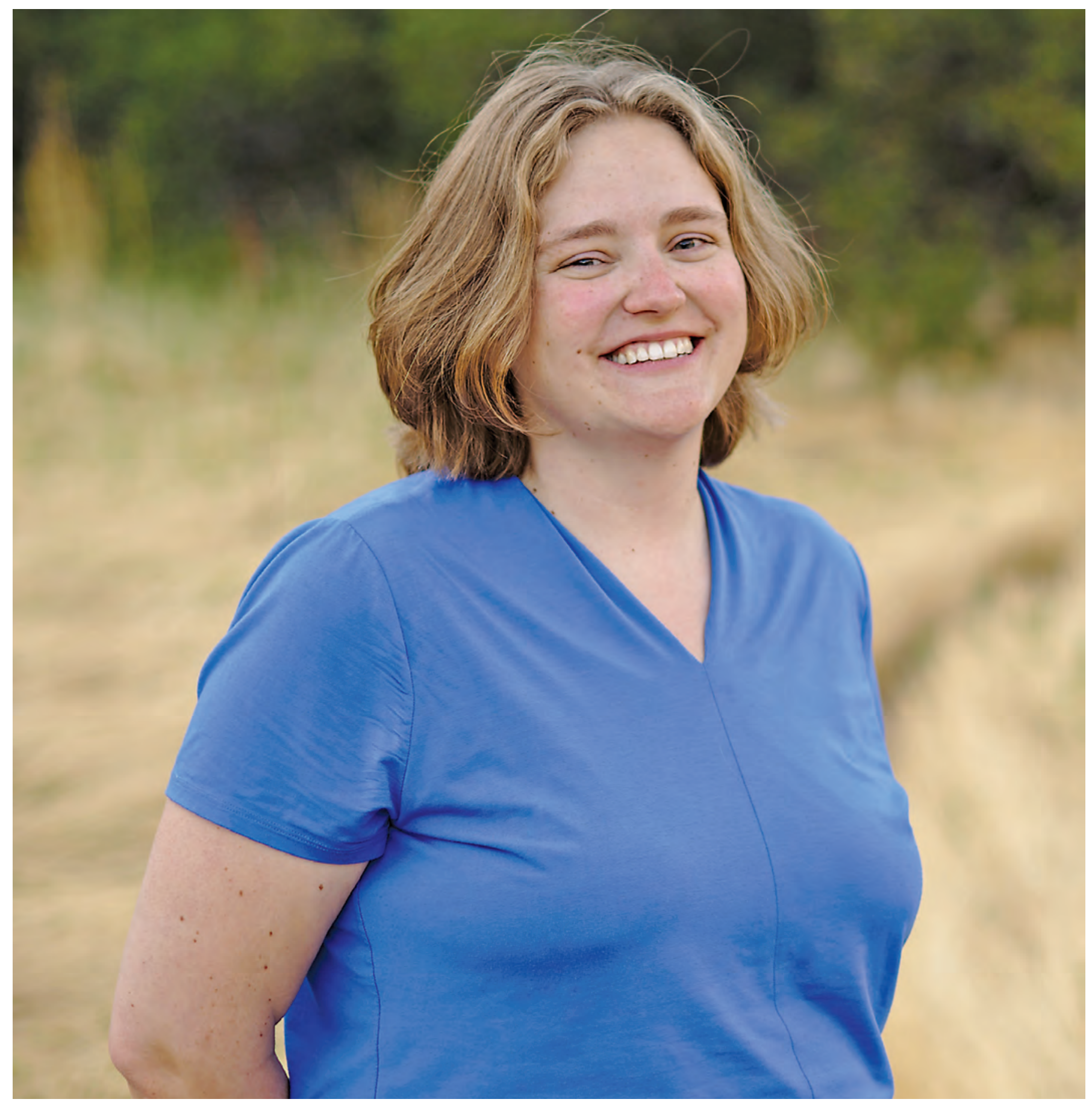




\section{WOMEN'S}

\section{HISTORY}

MONTH

\section{Synopsis of Research}

The main focus of Melanie Matchett Wood's research is in number theory and algebraic geometry, but it also involves work in probability, additive combinatorics, random groups, and algebraic topology. Much of Wood's work is motivated by questions of arithmetic statistics: How many are there of various number theoretic objects and what proportion of those objects have specified behaviors? An important example is to understand the distribution of class groups of random number fields. She has developed new conjectures on this topic for the non-Abelian analogs of class groups and proven theorems toward these conjectures in the function field case. Her future goals include further utilizing the insight that geometry gives in function field analogs to uncover new structure in the class groups of number fields.

\section{Biography}

In high school, I was the first female to represent the United States at the International Mathematics Olympiad. I went to college at Duke, where I was a Putnam Fellow and received the AMS-MAA-SIAM Morgan Prize. Then, I spent a year at the University of Cambridge doing Part III of their Mathematical Tripos. I received my PhD from Princeton in 2009, and then was a Szegő Assistant Professor at Stanford and an American Institute of Mathematics Five-Year Fellow. Now, I am a professor at the University of Wisconsin-Madison. Since 2011, I have been working part-time in my faculty position while I also stay at home with my young children. I am a Packard Fellow for Science and Engineering, a Sloan Research Fellow, and a National Science Foundation CAREER award winner. I am the 2018 recipient of the AWM-Microsoft Research Prize in Algebra and Number Theory. I was in the first class of the Fellows of the American Mathematical Society.

\section{Advice to Young Women}

My advice to young women is to decide what you want out of mathematics and to talk to others who have done what you would like to do to find out how to get there.

\section{Comments on Women's History Month}

It is important for all young people to have images and stories of people they can relate to doing the things that they might want to do when they grow up. When a group is underrepresented, such as women in mathematics, we need to make a special effort to make sure these stories reach young people so they can see what is possible.

\section{Photo Credit}

Photo of Melanie Matchett Wood courtesy of Joe Rabinoff. 


\section{Early Women Mathematicians in Princeton}

\section{Margaret A. Readdy and Christine Taylor}

WOMEN'S

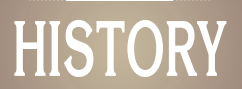

MONTH
In the early 1930s Anna Stafford, a graduate student at the University of Chicago interested in topology, asked to transfer to Princeton University to work with Oswald Veblen and James Alexander. She received a postcard from Princeton University saying "We don’t take girls.” In 1933 Veblen and Alexander became the first faculty members of the Institute for Advanced Study, along with Albert Einstein, John von Neumann, and Hermann Weyl. Upon finishing her PhD at Chicago, Stafford wrote to Veblen directly and was accepted as one of two women, the other being Mabel Schmeiser Barnes (PhD, 1931, Ohio State) in the inaugural class of seventeen IAS members in mathematics. Around the same time at the Institute, Emmy Noether was a visitor, commuting from Bryn Mawr College. Female mathematicians at IAS in the 1930s and 1940s also included those listed in the sidebar.

During this time there were no women students or faculty at Princeton University. In fact, women were not even allowed to audit courses. In 1954 Matsue Hanaoka visited the Princeton Mathematics Department as an International Rotary Fellow from Japan to work with Salomon Bochner in differential geometry upon the recommendation of Bochner's coauthor Kentaro Yano. Shiing-Shen Chern, a member at IAS, was also involved with inviting Hanaoka. Princeton's Graduate School admitted Hanaoka as a special student, not realizing that Hanaoka was a woman. Upon Hanaoka's arrival in Princeton, the Graduate School quickly found lodging for her outside the Graduate College. Princeton graduate students at the time Robert Gun-

Margaret A. Readdy is professor of mathematics at the University of Kentucky. Her email address is margaret. readdy@uky. edu.

Christine Taylor is senior lecturer of mathematics at Princeton University. Her email address is ctay lor@math . princeton . edu.

${ }^{1}$ Princeton Alumni Weekly, Volume 55, No. 10, November 26, 1954.

For permission to reprint this article, please contact:

reprint-permission@ams . org.

DOI: http://dx.doi.org/10.1090/noti1652
Mary Sinclair, PhD 1908, University of Chicago

Marie Johnson Yeaton, MS 1921, University of Iowa

Gertrude Stanley, PhD 1927, University of Oxford

Olga Taussky-Todd, PhD 1930, University of Vienna

Marie Charpentier, PhD 1931, University of Poitiers

Alice Lazerowitz, PhD 1932, University of Wisconsin, PhD 1938, University of Cambridge

Miriam Becker, PhD 1934, Yale University

Dorothy Manning Smiley, PhD 1937, Stanford University

Dorothy Maharam, PhD 1940, Bryn Mawr College

Sheila Power, PhD 1941, University of Edinburgh

Mary Dolciani, PhD 1947, Cornell University

Cécile DeWitt-Morett, PhD 1947, University of Paris

Verena Huber-Dyson, PhD 1947, University of Zürich

ning and Joseph Kohn remember Hanaoka as hardworking. She attended courses and tea regularly, and was perhaps not even aware that Princeton was not co-educational. In March 1955, Hanaoka along with Gunning and Kohn were driven by another graduate student to New York to attend a seminar. The car flipped over at a sharp turn, and all the passengers were thrown out. Hanaoka received the worst,

${ }^{2}$ Communications from Robert Gunning and Joseph Kohn; Princeton Town Topics Archive i a600307. us . archive . org/11/items/ towntopicsprince952unse/towntopicsprince952unse.pdf. 


\section{PRINCETON UNIVERSITY Mathematics Graduate Students I968}

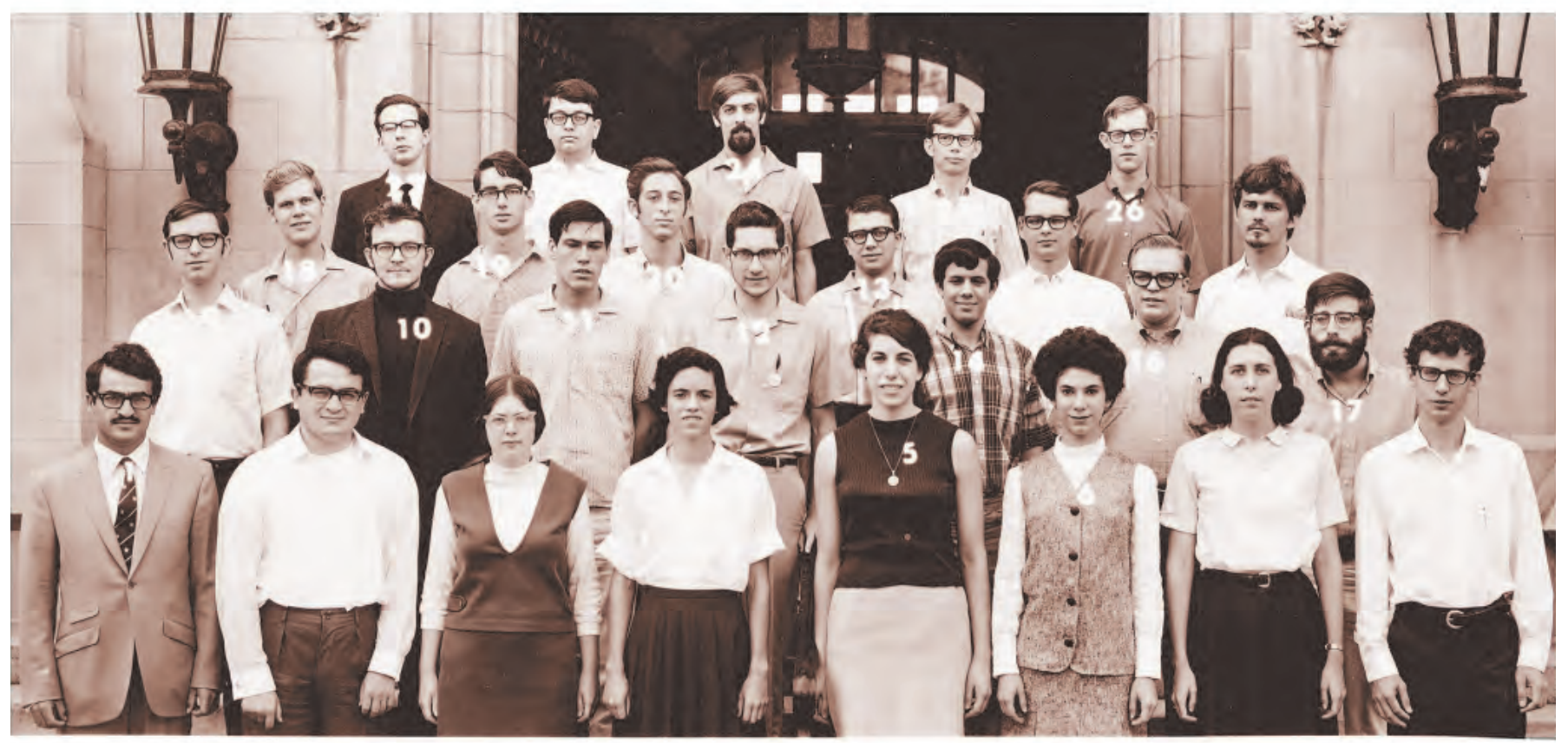
1) Mehmet E. Bozhüyük
2) Ibrahim Dibag
3) Bonnie Gold
4) Helen Hunt
5) Martha Katzin
6) Deborah L. Goldsmith
7) Majorie L. Stein
8) Neil K. Cohen
9) Harry Fedler

10) William J. Older

11) Brian K. Schmidt

12) Lawrence J. Dickson

13) John M. Masley

14) Mark L. Green

15) Edward P. Culp

16) James $C$. Thorpe

17) Charles N. Friedman

18) Gerald B. Folland
19) Anthony J. O'Conner
20) Robert Ephriam
21) Robert J. Jackson
22) Leonard M. Lipschitz
23) Daniel H. Saracino
24) Craig Benham
25) Kenneth E. Dahlberg
26) V. Alan Norton

Figure 1. In 1968 five woman graduate students entered Princeton's math department.

a head injury. She stayed in a hospital for a few weeks and returned to Japan before the end of the academic year. ${ }^{2}$

A decade later amid the Vietnam War, Princeton very slowly started admitting women into its graduate school, and finally women at the undergraduate level in 1969. In 1968, Princeton's math department admitted women graduate students for the first time (see Figure 1). Elias Stein, then chair of the math department, decided there should be a critical mass of female graduate students for them to be successful. Five women entered Princeton's math department in fall 1968: Bonnie Gold, Deborah L. Goldsmith, Helen Hunt, Martha Katzin, and Marjorie L. Stein (no relation to Elias Stein). Goldsmith and Stein each received a PhD in 1972, Katzin in 1974. Gold transferred to Cornell and received a PhD in 1976, and Hunt did not finish her $\mathrm{PhD}$.
In 1969, Yoko Nakagawa was the only woman admitted into the Princeton $\mathrm{PhD}$ program; she received her $\mathrm{PhD}$ in 1973 and returned to Japan. In 1973, Joan Birman became a visitor to the Princeton math department, commuting from home in New Rochelle while a mother of three teenagers. Birman's research monograph Braids, Links, and Mapping Class Groups, based on a course she gave in Princeton, is still a classic reference for topologists. ${ }^{3}$

\section{Photo Credit}

Figure 1 courtesy of the Department of Mathematics, Princeton University.

\footnotetext{
${ }^{3}$ See Joan Birman feature in the current Notices issue (page 250).
} 


\section{5 \\ Women's mathematics research}

Make a gift to an endowment that supports the work of women mathematicians.

The Joan and Joseph Birman Fellowship for Women Scholars gives exceptionally talented women extra research support during their mid-career years.

The Ruth Lyttle Satter Prize recognizes an outstanding contribution to mathematics research by a woman in the previous six years.

\section{Ruth Lyttle Satter Prize winners include:}

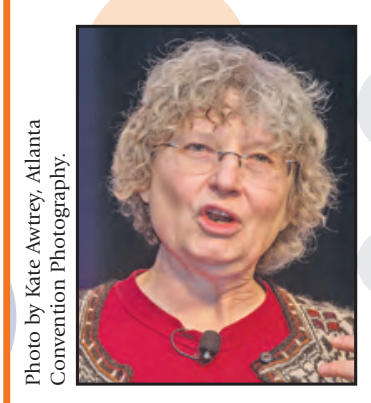

Ingrid Daubechies: analysis of wavelets and their applications

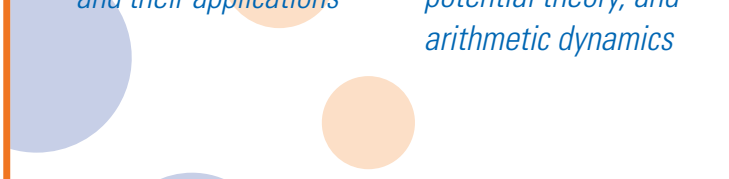

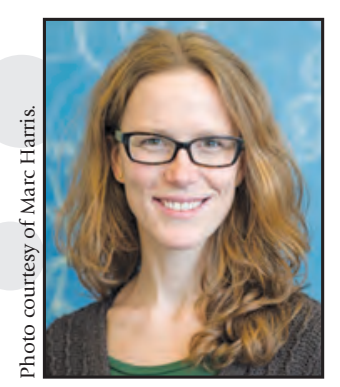

Laura DeMarco: complex dynamics, potential theory, and arithmetic dynamics

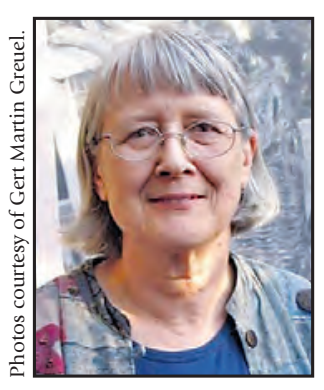

Dusa McDuff: symplectic geometry and topology

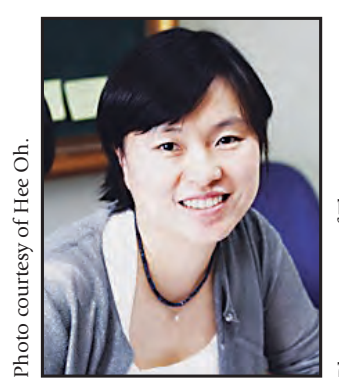

Hee Oh:

dynamics, Lie groups, geometry, and number theory

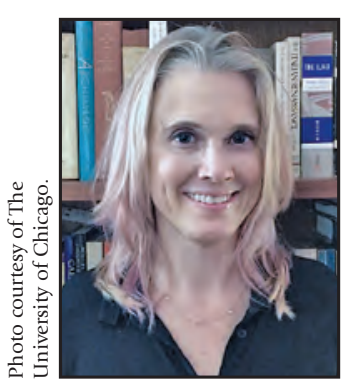

Amie Wilkinson: ergodic theory, smooth dynamical systems, and geometry

\section{To learn more and give visit wWw.ams.org/giving.}

\section{THANK YOU}

$$
\text { : AMS MMRBCAN }
$$

AMS Development Office 401.455.4111 development@ams.org 


\section{SPRING CENTRAL SECTIONAL SAMPLER}

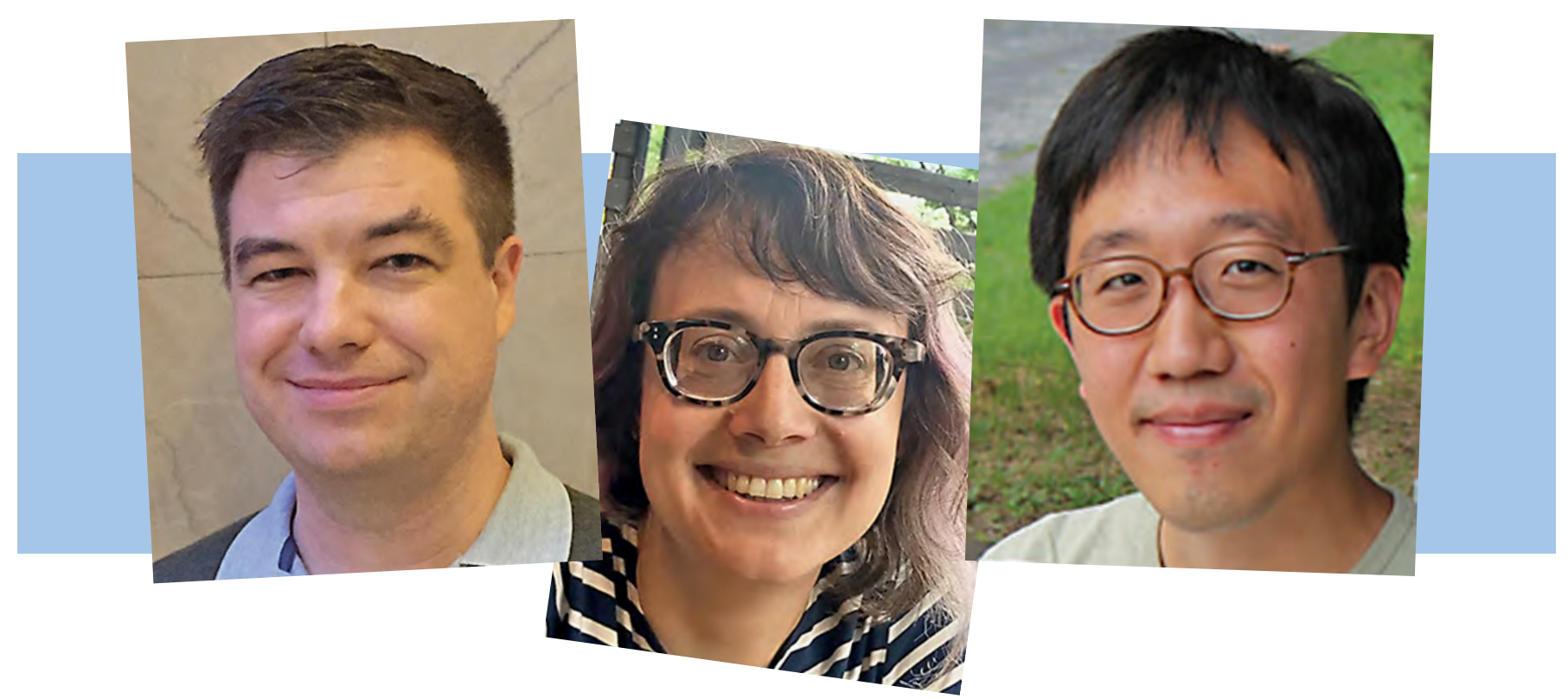

From left to right: Aaron Brown, Tullia Dymarz, and June Huh.

In this sampler, the speakers above have kindly provided introductions to their Invited Addresses for the upcoming AMS Spring Central Sectional Meeting.

\section{March 17-18, 2018 \\ (Saturday-Sunday) \\ The Ohio State University \\ Columbus, $\mathrm{OH}$}

Recent Progress in the Zimmer Program by Aaron Brown (University of Chicago) page 308

Bi-Lipschitz Equivalence of Groups by Tullia Dymarz (University of WisconsinMadison)

page 310

The Correlation Constant of a Field by June Huh (Institute for Advanced Study) page 311

For permission to reprint this article, please contact: reprint-permission@ams .org.

DOI: http://dx.doi.org/10.1090/noti1671

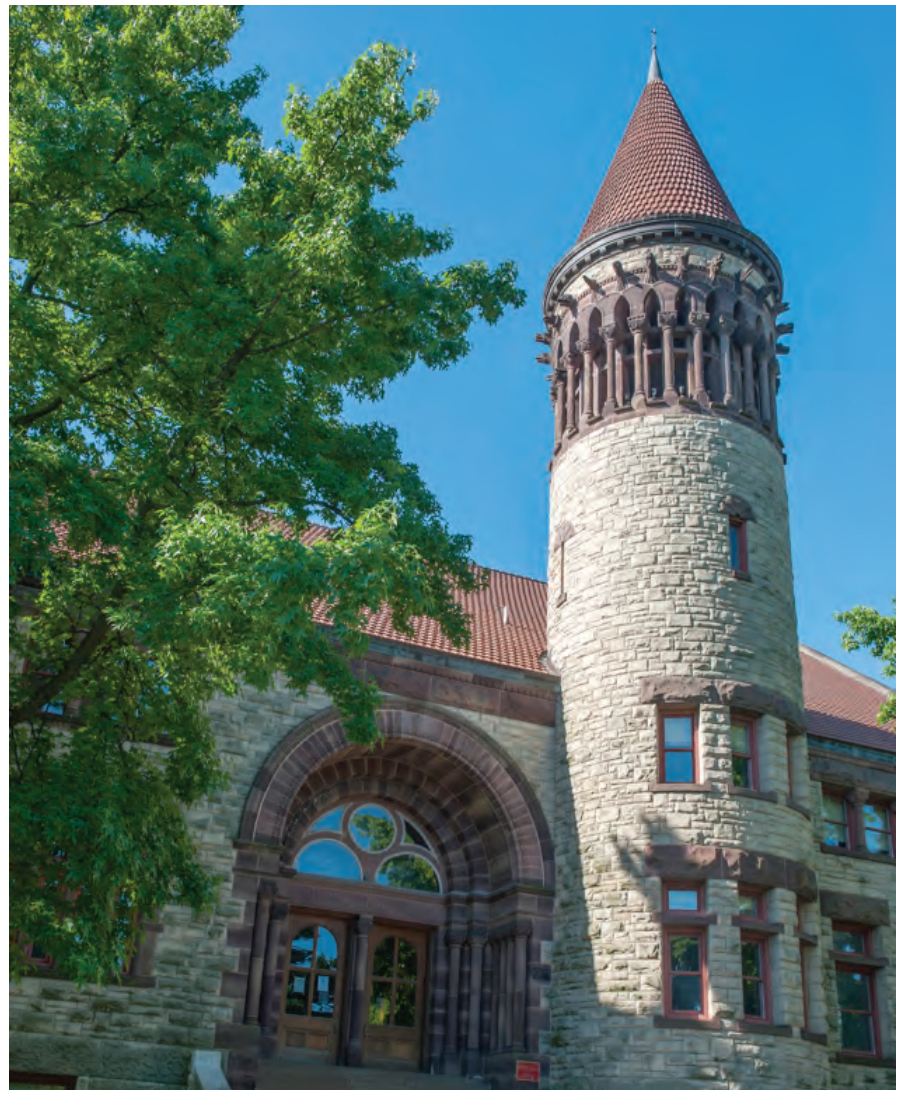




\section{Aaron Brown}

\section{Recent Progress in the Zimmer Program}

ABSTRACT. The Zimmer program refers to a number of questions and conjectures posed by Robert Zimmer in the 1980s concerning smooth actions of lattices in higher-rank Lie groups. We report on some recent progress by the author and collaborators.

\section{Lattices in Higher-Rank Lie Groups}

The primary objects in my talk are lattices $\Gamma$ in higher-rank simple Lie groups $G$.

The simplest example of such a lattice is $\Gamma=\operatorname{SL}(n, \mathbb{Z})$, the group of $n \times n$ integral matrices with determinant one. The group $\Gamma=\operatorname{SL}(n, \mathbb{Z})$ is a discrete subgroup of $G=\operatorname{SL}(n, \mathbb{R})$, the group of $n \times n$ invertible matrices of determinant one. It is well known that the coset space $G / \Gamma$ admits a finite-volume form which is invariant under the (left) action of $G$ by translations on $G / \Gamma$. In general, a lattice in a Lie group $G$ is a discrete subgroup $\Gamma \subset G$ such that the coset space $G / \Gamma$ has a finite $G$-invariant volume. A lattice is cocompact if the coset space $G / \Gamma$ is compact. The primary example of a lattice subgroup, $\operatorname{SL}(n, \mathbb{Z})$ in $\operatorname{SL}(n, \mathbb{R})$, is not cocompact; there do, however, exist cocompact lattices in $\operatorname{SL}(n, \mathbb{R})$.

The rank of the group $G=\operatorname{SL}(n, \mathbb{R})$ is $n-1$; this is the dimension of the subgroup

$$
A=\left\{\operatorname{diag}\left(e^{t_{1}}, e^{t_{2}}, \ldots, e^{t_{n}}\right)\right\}
$$

of diagonal matrices with positive entries. (Note that since we impose that the determinant is 1 , we have $t_{1}+t_{2}+\cdots+t_{n}=0$.) When $n \geq 3$, the rank of $G=\operatorname{SL}(n, \mathbb{R})$ is at least 2, and we say that $G$ is higher rank.

\section{Rigidity of Linear Representations}

Given a discrete group $\Gamma$, a linear representation of $\Gamma$ is a homomorphism $\pi: \Gamma \rightarrow \operatorname{GL}(d, \mathbb{R})$ from $\Gamma$ to a linear group $G L(d, \mathbb{R})$. When $\Gamma$ is a lattice in a higher-rank simple Lie group, it is well known that linear representations $\pi: \Gamma \rightarrow \operatorname{GL}(d, \mathbb{R})$ exhibit a number of strong rigidity properties. Early rigidity results include the local rigidity results due to Selberg and Weil and the global rigidity results due to Mostow, Margulis, and Prasad.

The strongest rigidity results for linear representations are the superrigidity and arithmeticity theorems of Margulis. Margulis's superrigidity theorem roughly states that any linear representation $\pi: \Gamma \rightarrow G L(d, \mathbb{R})$ of a lattice $\Gamma$ in a higher-rank simple Lie group $G$ extends to a representation $\tilde{\pi}: G \rightarrow \mathrm{GL}(d, \mathbb{R})$ "up to a compact error."

Aaron Brown is assistant professor of mathematics at the University of Chicago. His email address is awb@uchicago.edu.

For permission to reprint this article, please contact:

reprint-permission@ams . org.

DOI: http://dx.doi.org/10.1090/noti1643
In particular, since representations $\tilde{\pi}: G \rightarrow \mathrm{GL}(d, \mathbb{R})$ are fully classified, the superrigidity theorem essentially classifies all linear representations of lattices in higher-rank Lie groups. As a motivating result for my talk, we have the following corollary of Margulis's superrigidity theorem:

Corollary. For $n \geq 3$ and $d<n$, let $\Gamma$ be a lattice in $\operatorname{SL}(n, \mathbb{R})$. Then the image of any linear representation

$$
\pi: \Gamma \rightarrow \operatorname{GL}(d, \mathbb{R})
$$

is a finite group.

\section{Actions on Manifolds and the Zimmer Program}

Given a compact manifold $M$, let $\operatorname{Diff}(M)$ denote the group of smooth diffeomorphism of $M$. Given a fixed volume form on $M$, let $\operatorname{Diff}_{\mathrm{vol}}(M)$ be the group of volumepreserving diffeomorphisms of $M$. A smooth action of $\Gamma$ on $M$ (or a "nonlinear representation") is simply a homomorphism

$$
\alpha: \Gamma \rightarrow \operatorname{Diff}(M)
$$

Consider $\Gamma=\operatorname{SL}(n, \mathbb{Z})$. We describe two prototype actions of $\Gamma$ on low-dimensional compact manifolds; both examples are derived from the natural action of $\operatorname{SL}(n, \mathbb{Z})$ by linear transformations on $\mathbb{R}^{n}$. First, note that matrix multiplication preserves rays in $\mathbb{R}^{n}$. Thus, viewing the sphere $S^{n-1}$ as a parametrization of all rays in $\mathbb{R}^{n}$, we obtain a natural action of $\Gamma=\operatorname{SL}(n, \mathbb{Z})$ on $S^{n-1}$. Concretely, considering $S^{n-1}$ as the set of unit vectors in $\mathbb{R}^{n-1}$, we define $\alpha: \Gamma \rightarrow \operatorname{Diff}\left(S^{n-1}\right)$ by

$$
\alpha(\gamma)(x)=\frac{\gamma \cdot x}{\|\gamma \cdot x\|} .
$$

This action does not preserve any volume form on $S^{n-1}$. For the second action, since for every $\gamma \in \operatorname{SL}(n, \mathbb{Z})$ the coefficients of $\gamma$ are integral and det $\gamma=1$, the action of $\Gamma=\operatorname{SL}(n, \mathbb{Z})$ on $\mathbb{R}^{n}$ by linear transformations preserves the subgroup $\mathbb{Z}^{n} \subset \mathbb{R}^{n}$; this induces an action of $\Gamma=\operatorname{SL}(n, \mathbb{Z})$ on the $n$-dimensional torus $\mathbb{T}^{n}=\mathbb{R}^{n} / \mathbb{Z}^{n}$. Concretely, we define $\alpha: \Gamma \rightarrow \operatorname{Diff}\left(\mathbb{T}^{n}\right)$ by

$$
\alpha(\gamma)\left(x+\mathbb{Z}^{n}\right)=\gamma x+\mathbb{Z}^{n} .
$$

This action of $\operatorname{SL}(n, \mathbb{Z})$ preserves the natural Lebesgue volume form on $\mathbb{T}^{n}$.

The Zimmer program refers to a number of questions and conjectures posed by Robert Zimmer in the 1980s concerning smooth actions of lattices in higher-rank Lie groups. Roughly, these questions and conjectures aim to establish analogues of known rigidity results for linear representations in the "nonlinear" setting of smooth actions on manifolds. In particular, one may expect that all smooth actions of higher-rank lattices $\Gamma$ are constructed from standard algebraic actions (such as the actions described in the previous paragraph) and thus, in some sense, can be completely classified.

A first step towards classifying all actions is Zimmer's conjecture, which asserts that no nontrivial ${ }^{1}$ actions exist

\footnotetext{
${ }^{1}$ An action $\alpha: \Gamma \rightarrow \operatorname{Diff}(M)$ is trivial if the image $\alpha(\gamma) \subset \operatorname{Diff}(M)$ is a finite group; otherwise, it is said to be nontrivial.
} 


\section{SPRING CENTRAL SECTIONAL SAMPLER}

on closed manifolds whose dimension is less than the dimension of any manifold appearing in an algebraic action. For instance, when $\Gamma=\operatorname{SL}(n, \mathbb{Z})$, the action on the sphere $S^{n-1}$ is smallest dimensional algebraic action and the action on the torus $\mathbb{T}^{n}$ is smallest dimensional volumepreserving algebraic action. In particular, for lattices in $\operatorname{SL}(n, \mathbb{R})$, Zimmer's conjecture asserts the following.

Zimmer's Conjecture. For $n \geq 3$, let $\Gamma \subset \operatorname{SL}(n, \mathbb{R})$ be a lattice and let $M$ be a closed manifold.

(1) If $\operatorname{dim}(M)<n-1$, then any homomorphism

$$
\alpha: \Gamma \rightarrow \operatorname{Diff}^{r}(M)
$$

has finite image.

(2) If $\operatorname{dim}(M)<n$ and $\alpha$ preserves a smooth volume form, then any homomorphism

$$
\alpha: \Gamma \rightarrow \operatorname{Diff}_{\mathrm{vol}}^{r}(M)
$$

has finite image.

In particular, Zimmer's conjecture provides an analogue in the context of smooth actions of the finiteness of all linear representations given by the corollary to Margulis's superrigidity theorem above. Prior work towards Zimmer's conjecture primarily focused on actions on the circle and on surfaces, including work by Witte, Burger and Monod, Ghys, Polterovich, and Franks and Handel.

\section{Recent Progress in the Zimmer Program}

My talk will focus on recent work on Zimmer's conjecture and the larger Zimmer program due to David Fisher, Sebastian Hurtado, Federico Rodriguez Hertz, Zhiren Wang, and me.

The first main result is the verification of Zimmer's conjecture for actions of certain lattices in $\operatorname{SL}(n, \mathbb{R})$. Namely, when $\Gamma \subset \operatorname{SL}(n, \mathbb{R})$ is either

- a cocompact lattice in $\operatorname{SL}(n, \mathbb{R})$ or

- a finite-index subgroup of $\operatorname{SL}(n, \mathbb{Z})$,

Fisher, Hurtado, and I verified that Zimmer's conjecture holds. We also give the conjectured dimension bounds (below which all actions are trivial) for actions of cocompact lattices in certain other matrix groups, including $\mathrm{Sp}(2 n, \mathbb{R}), \mathrm{SO}(n, n)$, and $\mathrm{SO}(n, n+1)$. We also give partial results for actions of cocompact lattices in all higher-rank simple Lie groups which show triviality of all actions on manifolds whose dimension is below a certain critical dimension which grows linearly in the rank but which may be lower than the conjectured critical dimension.

At (and above) the critical dimensions $(n-1)$ and $n$ appearing in Zimmer's conjecture, there are examples of nontrivial algebraic actions of $\operatorname{SL}(n, \mathbb{Z})$. The aim of the Zimmer program is to classify all such smooth actions in terms of algebraic actions. Most results towards such a classification concern volume-preserving Anosov actions on tori (and nilmanifolds). These include a number of results by Katok, Lewis, Margulis, Qian, and Zimmer showing local and global rigidity of volume-preserving Anosov actions of higher-rank lattices on tori.

In recent work with Rodriguez Hertz and Wang, for $n \geq 3$ we study actions of $\Gamma=\operatorname{SL}(n, \mathbb{Z})$ (and more general higher-rank lattices) on tori $\mathbb{T}^{d}$ (and nilmanifolds). Under certain dynamical hypotheses on the induced action on homology and a mild lifting condition, we show that such an action is, up to a continuous surjective (but possibly noninvertible) change of coordinates, given by an algebraic action. For Anosov actions, the change of coordinates is then shown to be a smooth diffeomorphism. Unlike earlier approaches, our method does not require that the action preserve a volume (or any probability measure).

In another recent and ongoing work with Rodriguez Hertz and Wang, for $n \geq 3$ we study actions of $\Gamma=\operatorname{SL}(n, \mathbb{Z})$ on $(n-1)$-dimensional manifolds. For such actions, we showed that all nontrivial actions are measurably equivalent to (a finite measurable cover of) the standard action on the sphere; in ongoing work, we aim to show all such actions are equivalent to the natural action on the sphere (or projective space) up to a smooth change of coordinates.

The proofs of the above results combine tools and results from many areas of mathematics, including smooth ergodic theory, homogeneous dynamics, Lie theory, representation theory, and operator algebras. In my talk I will present a number of motivations for the Zimmer program, outline the new results discussed above, and indicate how tools and ideas from various areas of mathematics are combined to establish these results.

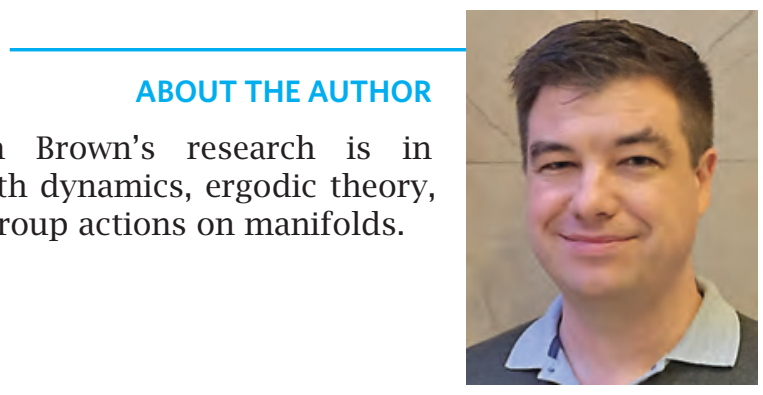

Aaron Brown 


\section{Tullia Dymarz}

\section{Bi-Lipschitz Equivalence of Groups}

ABSTRACT. We consider the difference between biLipschitz equivalence and quasi-isometric equivalence of infinite finitely generated groups.

The foundational premise of geometric group theory is that a finitely generated group can be endowed with a canonical metric, unique up to equivalence. This equivalence class should contain all word metrics (those metrics given by fixing a finite generating set and counting the number of generators it takes to transform one element to the other), but it may contain many other kinds of metrics. Gromov started the field of geometric group theory by suggesting that the right equivalence to study is quasi-isometry. A quasi-isometric equivalence between metric spaces $\left(X, d_{X}\right),\left(Y, d_{Y}\right)$ requires the existence of a map $f: X \rightarrow Y$ and two constants $K, C$ such that for all $x, x^{\prime} \in X$,

$$
-C+\frac{1}{K} d_{X}\left(x, x^{\prime}\right) \leq d_{Y}\left(f(x), f\left(x^{\prime}\right)\right) \leq K d_{X}\left(x, x^{\prime}\right)+C
$$

and such that the $C$ neighborhood of the image of $f$ is all of $Y$. This last condition implies that the property is symmetric.

It is not hard to check that all word metrics on a fixed group are quasi-isometric. In addition, many finitely generated groups that arise in geometry, such as fundamental groups of compact Riemannian manifolds, are quasi-isometric to well-studied metric spaces on which they act by isometries, such as the universal cover of the manifold. This phenomenon is captured by MilnorŠvarc's fundamental lemma of geometric group theory: If a group acts properly discontinuously and cocompactly on a proper geodesic metric space then it is quasi-isometric to the metric space it acts on.

The question now is, which groups are quasi-isometric to each other? A simple exercise shows that finite-index subgroups are always quasi-isometric, but there are many examples of groups that are quasi-isometric that do not share any finite-index subgroups. In addition, certain properties that are defined algebraically actually turn out to be geometric, in that they are preserved by quasiisometry. The prime example of this theme is Gromov's result from the 1980 s that shows that any group quasiisometric to a nilpotent group (nilpotence is a condition defined algebraically) must itself contain a finite-index nilpotent subgroup.

Tullia Dymarz is associate professor of mathematics at the University of Wisconsin-Madison. She is supported by NSF CAREER grant DMS-1552234. Her email address is dymarz @math.wisc.edu.

For permission to reprint this article, please contact: reprint-permission@ams . org.

DOI: http://dx.doi.org/10.1090/noti1644

My primary research focus has been on the quasiisometric classification of polycyclic groups. Algebraically, polycyclic groups lie between nilpotent groups (a geometric class of groups) and solvable groups (not a geometric class of groups). In 2007 Eskin-Fisher-Whyte showed that all groups quasi-isometric to the smallest nonnilpotent polycyclic group are also, up to finite-index, polycyclic and conjectured that being polycyclic is indeed a geometric property. This work is technical and will not be the subject of my talk.

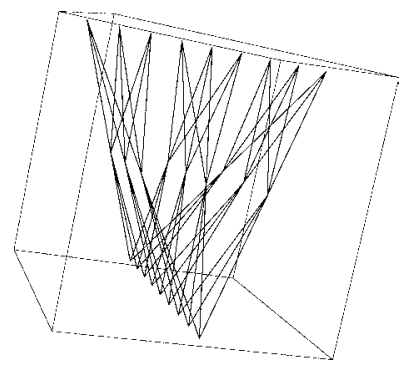

Figure 1. A portion of the Cayley graph for the lamplighter group $(\mathbb{Z} / 3 \mathbb{Z}) \backslash \mathbb{Z}$, which is quasi-isometric but not bi-Lipschitz equivalent to its index-two subgroup $(\mathbb{Z} / 3 \mathbb{Z} \times \mathbb{Z} / 3 \mathbb{Z})$ ? $\mathbb{Z}$.

Instead I will talk about another natural equivalence on finitely generated groups which is defined similarly to quasi-isometry except that the additive constant $C$ is required to be zero. This restriction gives us bi-Lipschitz equivalence. Under this equivalence the fundamental lemma no longer holds, but all word metrics are biLipschitz equivalent, and surprisingly for certain classes of groups, such as nonamenable groups, as proved by Whyte, both types of maps generate the same equivalence. This equivalence was also considered by Gromov, who asked whether it was possible to find groups that are quasi-isometric but not bi-Lipschitz equivalent. In 2010 I found the first examples of such groups. These groups come from the family of so-called lamplighter groups, a portion of whose Cayley graphs can be seen in Figure 1.

Incidentally, these examples can be chosen to be a group and one of its finite-index subgroups. Unfortunately there have been very few examples since then, and it is unclear where else to look for them. In my talk I will explore bi-Lipschitz equivalence not only of groups but also of other discrete metric spaces.

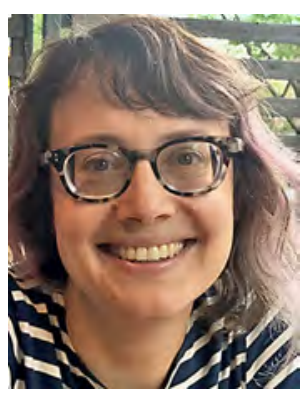

Tullia Dymarz

\section{ABOUT THE AUTHOR}

Tullia Dymarz works in geometric group theory with a focus on the large scale geometry of solvable groups. 


\section{June Huh}

\section{The Correlation Constant of a Field}

Let $G$ be a finite connected graph, let $i, j$ be distinct edges, and let $T$ be a random spanning tree of $G$. The probability that $i$ is in $T$ can only decrease by assuming that $j$ is in $T$ :

$$
\operatorname{Pr}(i \in T) \geq \operatorname{Pr}(i \in T \mid j \in T) .
$$

In other words, the number $b_{-}$of spanning trees containing given edges satisfies

$$
\frac{b_{i}}{b} \geq \frac{b_{i j}}{b_{j}} .
$$

Now let $E$ be a finite spanning subset of a vector space $V$, let $i, j$ be distinct nonzero vectors in $E$, and write $b_{-}$ for the number of bases in $E$ containing given vectors. Do we still have

$$
\frac{b_{i}}{b} \geq \frac{b_{i j}}{b_{j}} ?
$$

In 1974 Paul Seymour and Dominic Welsh found the first example of a vector configuration over a field of characteristic 2 with $\frac{b b_{i j}}{b_{i} b_{j}}=\frac{36}{35}$ for some $i$ and $j$. How large can the ratio be?

Definition. The correlation constant of a field $k$ is the supremum of $\frac{b b_{i j}}{b_{i} b_{j}}$ over all pairs of distinct nonzero vectors $i$ and $j$ in finite vector configurations in vector spaces over $k$.

This may be an interesting invariant of a field. While studying Hodge-Riemann relations for the intersection cohomology of certain projective varieties, Botong Wang and I noticed that

$$
\frac{b b_{i j}}{b_{i} b_{j}}<2
$$

for any vector configuration. Thus the correlation constant of any field is at most 2 . What is the correlation constant of, say, $\mathbb{Z} / 2 \mathbb{Z}$ ? Does the correlation constant really depend on the field? We discuss these and other questions.
June Huh is visiting professor of mathematics at the Institute for Advanced Study. His email address is junehuh@ias. edu.

For permission to reprint this article, please contact:

reprint-permission@ams.org.

DOI: http://dx.doi.org/10.1090/noti1645

\section{ABOUT THE AUTHOR}

June Huh works at the interface between combinatorics and algebraic geometry.

\section{Image Credits}

Photo of Aaron Brown courtesy of Aaron Brown.

Photo of Tullia Dymarz courtesy of Talia Fernos, associate professor of mathematics, University of North Carolina, Greensboro.

Dymarz Figure 1 by Tullia Dymarz.

Photo of June Huh courtesy of Woo-Hyun Kim.

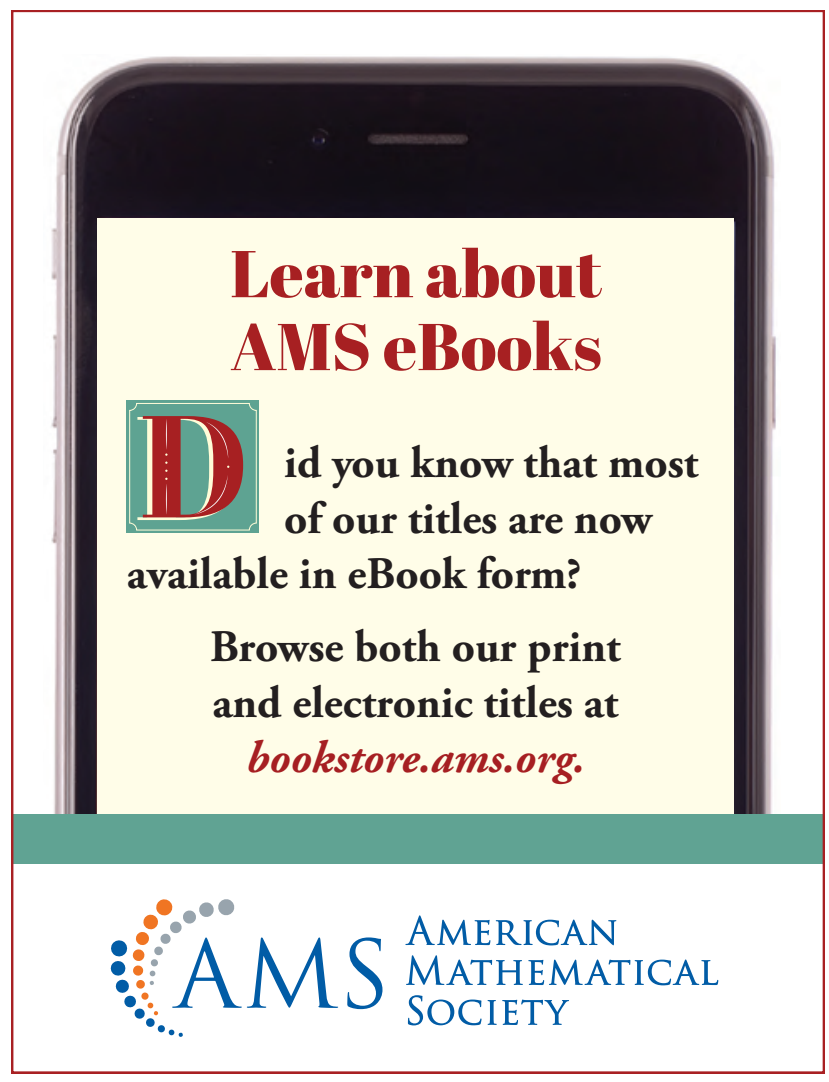




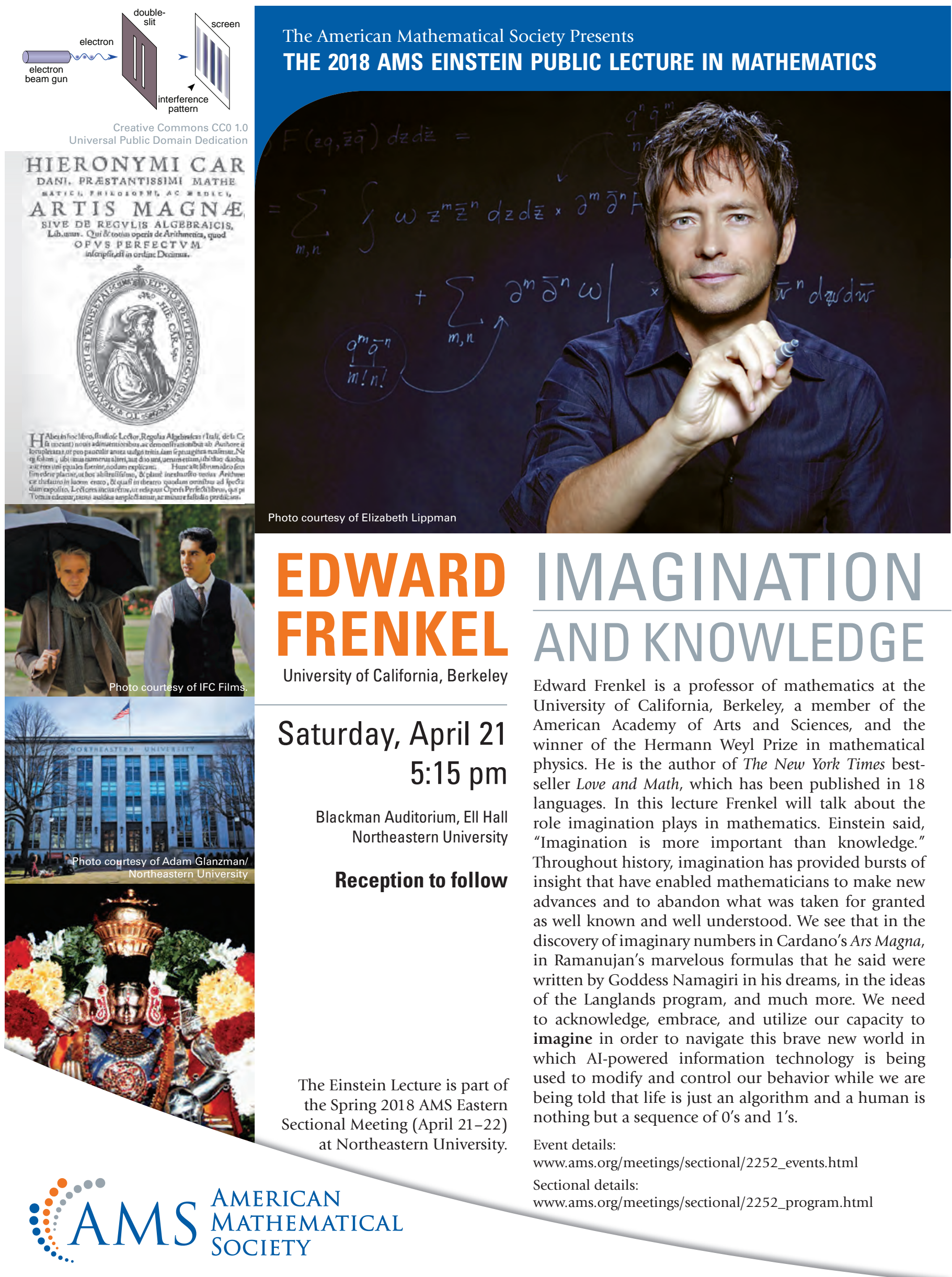




\title{
Open Problems Concerning Michell Trusses
}

\author{
Wilfrid Gangbo \\ Communicated by Christina Sormani
}

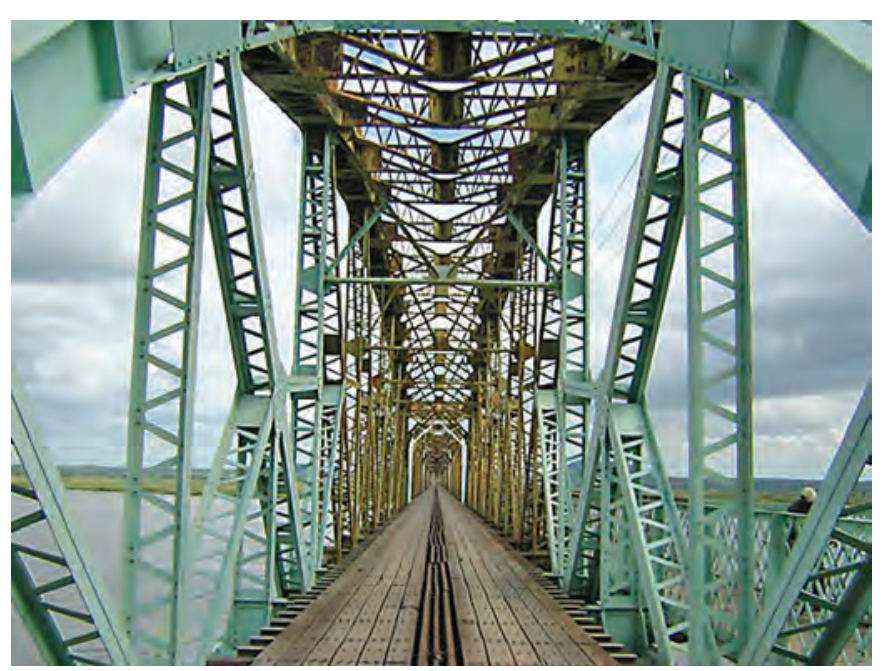

The Dona Ana Bridge in Sena, Mozambique.

We give a brief introduction to the problem of Michell trusses, a beautiful and challenging optimization problem related to the construction of bridges that was formulated by Michell [3] in 1904. Activity on the Michell Problem only began gaining momentum about fifty years later, and many interesting questions about it are still open. This problem first appeared in the engineering literature in a formulation that is accessible to any college student. It leads to deep and fascinating mathematical problems, and even the original relaxed problem is not yet fully resolved. It is a good illustration of the obstacles one must overcome when dealing with certain variational problems and of how duality can be a key to characterizing optima.

Our data consist of finitely many force vectors

$$
\mathbf{F}_{1}, \ldots, \mathbf{F}_{k} \in \mathbb{R}^{d},
$$

Wilfrid Gangbo is professor of mathematics at UCLA and an AMS Fellow who has served as the Eisenbud Chair at MSRI/UC Berkeley. He acknowledges funding by the National Science Foundation. His email address is wgango@math. ucla. edu.

For permission to reprint this article, please contact: reprint-permission@ams.org.

DOI: http://dx.doi.org/10.1090/noti1648 where $d=2,3$, and their respective points of application

$$
M_{1}, \ldots, M_{k} \in \mathbb{R}^{d} .
$$

We refer to

$$
\mathbf{F}=\sum_{j=1}^{k} \mathbf{F}_{j} \delta_{M_{j}}
$$

as a force, which is assumed to be of null average and to have zero torque:

$$
\sum_{j=1}^{k} \mathbf{F}_{j}=0, \quad \sum_{j=1}^{k} \mathbf{F}_{j} \wedge M_{j}=0 .
$$

Such a force must have at least two points of application $(k \geq 2)$, and the simplest example is

$$
\text { beam }(A, B)=\left(\delta_{B}-\delta_{A}\right) \frac{B-A}{|B-A|},
$$

depicted in Figure 1, which represents a beam in tension. Alternatively, we have -beam $(A, B)$, which represents a beam in compression.

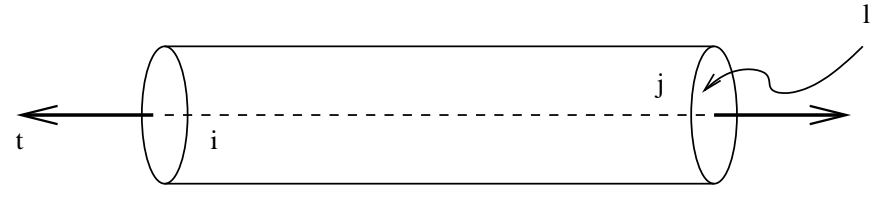

Figure 1. A beam in tension is represented by a force beam $(A, B)$.

An elementary fact in the mechanical engineering literature is that any force $\mathbf{F}$ that satisfies (1) can be decomposed into a finite linear combination of beam $\left(A_{i}, A_{j}\right)$ : there exist $n \in \mathbb{N}$, large enough, $\left\{A_{1}, \ldots, A_{n}\right\} \subset \mathbb{R}^{d}$, and $\left\{\lambda_{i j}\right\}_{i, j=1}^{n} \subset \mathbb{R}$ such that

$$
\mathbf{F}=\sum_{i, j=1}^{n} \lambda_{i j} \operatorname{beam}\left(A_{i}, A_{j}\right)
$$

When this equation holds we say that the frame $[\lambda, \mathcal{A}]=$ $\left[\left\{\lambda_{i j}\right\},\left\{A_{i}\right\}\right]$ withstands $\mathbf{F}$.

In general the decomposition in (2) is far from unique, and so one seeks the most optimal decomposition. Michell himself proposed $[\lambda, \mathcal{A}]$ to be optimal if it minimizes the 


\section{COMMUNICATION}

cost function

$$
\operatorname{Cost}[\lambda, \mathcal{A}]:=\sum_{i, j=1}^{n}\left|\lambda_{i j}\right|\left|A_{i}-A_{j}\right|,
$$

which represents the total volume of a frame, where $\left|A_{j}-A_{i}\right|$ is the length of the beam in the frame extending from $A_{i}$ to $A_{j}$ and having surface area $\left|\lambda_{i j}\right|$. By symmetrizing the problem one can (with no loss of generality) assume that the problem's matrix $\left(\lambda_{i j}\right)_{i j}$ is symmetric. The set $\mathcal{U}$ of displacements, $u: \mathbb{R}^{d} \rightarrow \mathbb{R}^{d}$, is defined as

(4) $\mathcal{U}=\left\{u:|(u(x)-u(y)) \cdot(x-y)| \leq|x-y|^{2}\right\}$.

The functional $J: \mathcal{U} \rightarrow \mathbb{R}$, which represents the total work done by the force $\mathbf{F}$ when the material undergoes displacement $u$, is defined by

$$
J[u]:=\int_{\mathbb{R}^{d}}\langle u ; \mathbf{F}(d x)\rangle,
$$

plays a crucial role. Indeed if $u \in \mathcal{U}$ and the frame $[\lambda, \mathcal{A}]$ withstands F, then the work may be computed to be

$$
J[u]=\sum_{i, j=1}^{n} \lambda_{i j}\left(u\left(A_{j}\right)-u\left(A_{i}\right)\right) \cdot\left(\frac{A_{j}-A_{i}}{\left|A_{j}-A_{i}\right|}\right) .
$$

So immediately we see that

$$
J[u] \leq \sum_{i, j=1}^{n}\left|\lambda_{i j}\right| \frac{\left|A_{j}-A_{i}\right|^{2}}{\left|A_{j}-A_{i}\right|}=\operatorname{Cost}[\lambda, \mathcal{A}] .
$$

Thus, the work is bounded above by the cost,

$$
\sup _{u \in \mathcal{U}} J[u] \leq \inf _{(\lambda, \mathcal{A}, n)}\{\operatorname{Cost}[\lambda, \mathcal{A}]\},
$$

where the infimum on the right is taken over frames $[\lambda, \mathcal{A}]$ that withstand $\mathbf{F}$ as in (2).

Michell [3] proposed that one should have the following duality principle:

$$
\sup _{u \in \mathcal{U}} J[u]=\inf _{(\lambda, \mathcal{A}, n)}\{\operatorname{Cost}[\lambda, \mathcal{A}]\} .
$$

Let us consider $d=2$ and the force

$$
\mathbf{F}=\left(\delta_{e_{1}}-2 \delta_{0}+\delta_{-e_{1}}\right),
$$

where $e_{1}=(1,0)$ and $e_{2}=(0,1)$. Let us consider a sequence of frames, $\left[\lambda^{n}, \mathcal{A}^{n}\right]$, as in Figure 2 .
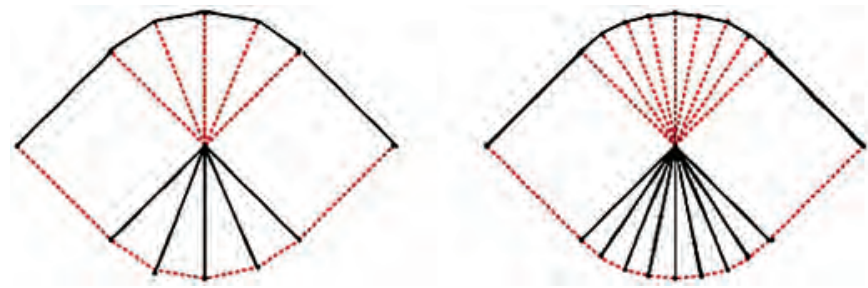

Figure 2. The frames $\left[\lambda^{n}, \mathcal{A}^{n}\right]$ when $n=13$ and $n=21$, where dotted red lines are in tension and thick black lines are in compression.

Bouchitté, Seppecher, and the author [1] identified a specific function $u_{*} \in \mathcal{U}$ and proved that

$$
J\left[u_{*}\right] \geq \operatorname{Cost}\left[\lambda^{n}, \mathcal{A}^{n}\right]-0\left(\frac{1}{n}\right) .
$$

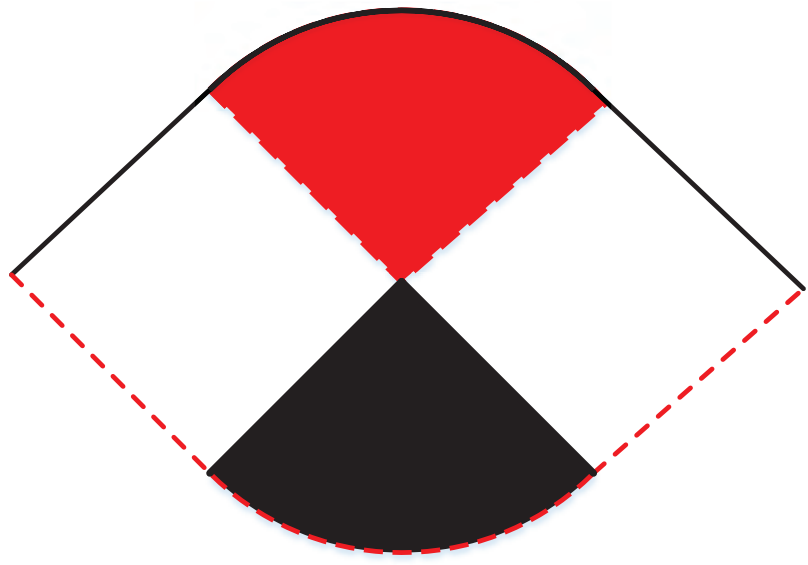

Figure 3. The limit measure $\sigma^{*}$.

By (7) and (9), not only is $\left(\left[\lambda^{n}, \mathcal{A}^{n}\right]\right)_{n}$ a minimizing sequence but (8) holds as well.

Therefore, if $u_{*}$ is a maximizer in (8), then $\left[\left\{\lambda_{i j}\right\},\left\{A_{i}\right\}\right]$ is a minimizer in (8) if and only if

$$
\lambda_{i j}\left(u\left(A_{j}\right)-u\left(A_{i}\right)\right) \cdot\left(A_{j}-A_{i}\right)=\left|\lambda_{i j}\right|\left|A_{j}-A_{i}\right|^{2}
$$

for all $i, j \in\{1, \ldots, n\}$.

Observe that as $n$ goes to $\infty$, the sequence $\left(\left[\lambda^{n}, \mathcal{A}^{n}\right]\right)_{n}$ intuitively "converges" to the measure $\sigma^{*}$, depicted in Figure 3, which clearly fails to belong to the set of frames made from finitely many beams. To understand this convergence more rigorously, we view each term in the sequence of frames as a measure:

$$
\sigma:=\sum_{i, j=1}^{n} \frac{\lambda_{i j}}{\left|A_{j}-A_{i}\right|^{2}}\left(A_{j}-A_{i}\right) \otimes\left(A_{j}-A_{i}\right) \mathcal{H}_{\mid\left[A_{i}, A-j\right]}^{1},
$$

where $\mathcal{H}_{\left[\left[A_{i}, A_{j}\right]\right.}^{1}$ denotes the Hausdorff measure restricted to the segment $\left[A_{i}, A_{j}\right]$. We can then take the weak limit of the measures to obtain $\sigma^{*}$. It is readily checked that the equilibrium equation (2) can be written in the class of measures as

$$
-\operatorname{div}(\sigma)=\mathbf{F}
$$

in the sense of distributions.

Thus in order to take into account all possible structures that may appear in the limit, we are forced to search for minimizers in the bigger set, $\Sigma$, of strain tensors:

$$
\Sigma=\{\sigma:-\operatorname{div}(\sigma)=\mathbf{F}\} .
$$

A strain tensor, $\sigma$, is a symmetric matrix whose entries are Radon measures satisfying (10) as distributions. Written in terms of strain tensors,

$$
\operatorname{Cost}[\lambda, \mathcal{A}]=C[\sigma]:=\int_{\mathbb{R}^{d}} \rho^{0}(\sigma),
$$

where $\rho^{0}$ is the one-homogeneous function that associates to a square symmetric matrix the sum of the absolute values of its eigenvalues. Since the infimum in (8) is taken over the set of all natural numbers $n \in \mathbb{N}$, it can be shown to have its infimum achieved and in fact

$$
\sup _{u \in \mathcal{U}} J[u]=\inf _{\sigma \in \Sigma} C[\sigma] .
$$


This right-hand side is what is referred to as a relaxation of the minimization problem at the right-hand side of (8).

In order to keep track of the stream lines of principal actions in the strain tensors, our proposal in [1] was to look for $\sigma$ that can be represented by signed Radon measures

$$
\gamma=\gamma^{+}-\gamma^{-}
$$

defined on the set of curves. The positive part $\gamma^{+}$of the signed measure corresponds to lines in tension, while the negative part $\gamma^{-}$corresponds to the lines in compression. We shall use the notation

$$
|\gamma|:=\gamma^{+}+\gamma^{-} \text {. }
$$

These stream lines form the Hencky-Prandtl net when $d=2$. See Figure 4 .

As described in [1], the Hencky-Prandtl net is a family of orthogonal curves which represent the limits of the families of bars through the optimization process.
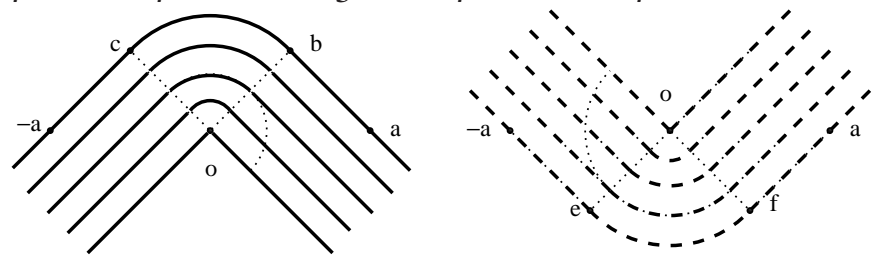

Figure 4. Here we have depicted the Hencky-Prandtl net for $\sigma *$ of Figure 3, with $\gamma^{+}$depicted on the left and $\gamma^{-}$on the right.

Let $\mathbf{X}$ be the set of $C^{1,1}$ curves of finite length and for $C \in \mathbf{X}$ let $t_{C}$ denote a unit tangent to $C$. Any Radon measure $\gamma$ on $\mathbf{X}$ induces a symmetric matrix $\sigma[\gamma] \in \Sigma$ defined as

$$
\sigma[\gamma]=\int_{\mathbf{X}}\left(\int_{C}\left\langle\xi(x) ; t_{C} \otimes t_{C}\right\rangle d \mathcal{H}^{1}\right) \gamma(d C) .
$$

We [1] show that the minimization problem in (11) is equivalent to finding the infimum

$$
\inf \left\{\int_{\mathbf{X}} \mathcal{H}^{1}(C)|\gamma|(d C):-\operatorname{div}(\sigma[\gamma])=\mathbf{F}\right\} .
$$

When (12) has a minimizer, $\gamma_{*}$, then the strain tensor, $\sigma\left[\gamma_{*}\right]$, is called a Michell truss.

Thanks to Korn's inequality, one sees that (11) admits a maximizer, $u_{*}$, which is almost everywhere differentiable. Given $C$ in $\mathbf{X}$, let $\kappa$ denote the curvature along $C$ and let $s$ be the arc-length parametrization. The tangential component of $u_{*}$, denoted as $u_{\tau}:=u_{*} \cdot t_{C}$, is Lipschitz along $C$, whereas the orthogonal component denoted as $u_{v}$ is continuous. We show that a necessary and sufficient condition for $\gamma_{*}$ to be a minimizer in (12) is

$$
\frac{d u_{\tau}}{d s}-\kappa u_{v}= \pm 1 \quad \mathcal{H}^{1} \text { a.e. and } \gamma^{ \pm} \text {a.e. }
$$

\section{Open Problems}

I. Does (12) admit a minimizer $\gamma_{*}$ ? When is $\sigma\left[\gamma_{*}\right]$ uniquely determined?
II. Is there a radius, $r>0$, such that any curve, $C$, in the support of $\gamma_{*}$ is contained in the ball $B_{r}(0)$ ? Are minimizers of $\inf _{\Sigma} C$ supported by $B_{r}(0)$ ?

III. Let $u_{*}$ be a maximizer of the functional $J[u]$ over the collection of displacements $u \in \mathcal{U}$, as in (4)-(5). Can one identify the set where

$$
2 E\left(u_{*}\right):=\nabla u_{*}+\nabla^{T} u_{*}
$$

is not continuous or not differentiable?

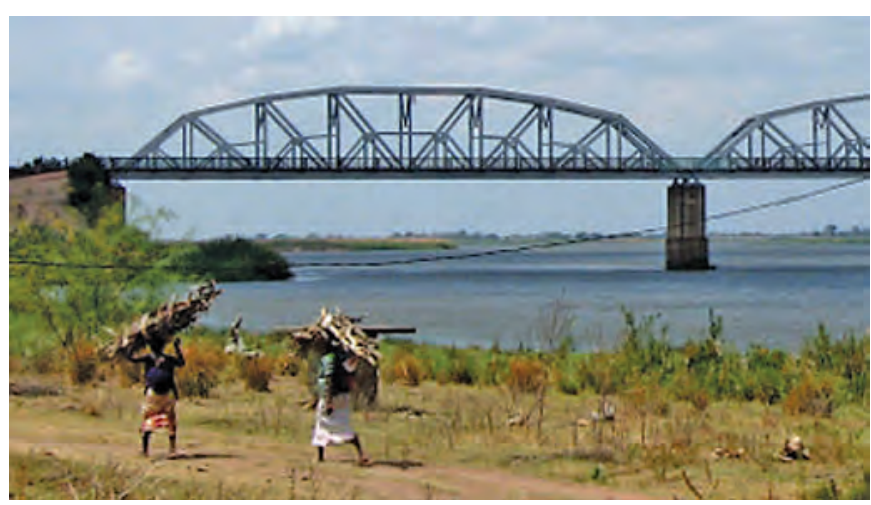

The Dona Ana Bridge in Africa has forty spans and is one of the longest bridges in the world.

We close with a discussion of the first of these open problems in dimension $d=2$. Note that $u_{*} \in \mathcal{U}$ if and only if the eigenvalues $e_{1}$ and $e_{2}$ of the symmetric matrix $E\left(u_{*}\right)$ have their ranges in the set $[-1,1]$. Let $a_{1}$ and $a_{2}$ be the eigenvectors of $E\left(u_{*}\right)$, associated to the eigenvalues $e_{1}$ and $e_{2}$ respectively, so that

$$
E\left(u_{*}\right)=e_{1} a^{1} \otimes a^{1}+e_{2} a^{2} \otimes a^{2} .
$$

Since $J[u]$ is a linear function of $u$, formally at least its maximizer $u_{*}$ is an extreme point of the convex set $\mathcal{U}$. One is tempted to assume that

$$
\left|e_{1}\right|=\left|e_{2}\right| \equiv 1 ;
$$

however this remains an open question in general.

As a symmetric matrix, $\sigma_{*}$ is also diagonalizable, and its eigenvalues $\lambda_{1}$ and $\lambda_{2}$ are signed Radon measures. The duality identity (8) not only forces $\sigma_{*}$ to have the same eigenvectors as $E\left(u_{*}\right)$ but also implies that

$$
e_{1} \lambda_{1} \geq 0 \text { and } e_{2} \lambda_{2} \geq 0 \text {. }
$$

Therefore, $\left(\lambda_{1}, \lambda_{2}\right)$ solves the system

$$
-\operatorname{div}\left(\lambda_{1} a^{1} \otimes a^{1}+\lambda_{2} a^{2} \otimes a^{2}\right)=\mathbf{F} .
$$

This is a system of hyperbolic equations in $\left(\lambda_{1}, \lambda_{2}\right)$ whose characteristics would be two families of orthogonal curves if we could prove these characteristics exist. These curves are the missing pieces to build a measure $\gamma_{*}$ such that $\sigma_{*}=\sigma\left[\gamma_{*}\right]$

It is suspected that if $e_{1} e_{2} \leq 0$ everywhere, then there is at most one pair $\left(\lambda_{1}, \lambda_{2}\right)$ that satisfies (13) on $\mathbb{R}^{2}$. This would solve the question of uniqueness in dimension two.

Those who are interested in learning more about these open problems should see the work of the author with Bouchitté and Seppecher [1], as well as his work with 
Computational Aspects of Time Dependent Electromagnetic Wave Problems in Complex Materials

June $25-29,2018$

\section{ORGANIZING COMMITTEE}

Vrushali Bokil, Oregon State University

Yingda Cheng, Michigan State University

Susan Hagness, University of Wisconsin, Madison

Fengyan Li, Rensselaer Polytechnic Institute

Fernando Teixeira, The Ohio State University

Shan Zhao, University of Alabama

\section{WORKSHOP DESCRIPTION}

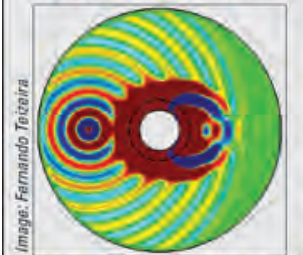

Forward simulations of the propagation and scattering of transient electromagnetic (EM) waves in complex media are important in a variety of applications, such as radar, environmental and medical imaging, noninvasive detection of cancerous tumors, design of engineered composites such as metamaterials, communication and computation, and global climate assessment, among others. These applications involve multiple spatial and temporal scales, complex geometries, spatial and temporal heterogeneities, and stochastic effects at small scales. In recent years, there has been an upsurge in the design and development of new materials with tailored EM properties under the conceptual umbrella of metamaterials. This workshop aims to bring together different scientific communities, including mathematicians, engineers, physicists, software developers and other relevant people, to disseminate current progress in their areas and develop potential collaborations to address challenges involved in the solution of the timedomain Maxwell's equations in complex materials through computational and experimental research with the broad aim of addressing and solving real-world applications.

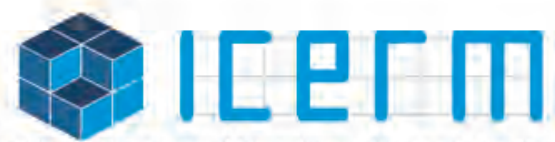

Institute for Computational and Experimental Research in Mathematics
Proposals being accepted: Semester Program Topical Workshop Small Group Research Program Summer Undergrad Program

ICERM is a National Science Foundation Mathematics Institute at Brown University in Providence, Rl.
Applications being accepted: Semester Program or Workshop Postdoctoral Fellowship

Sponsorships being accepted: Academic or Corporate

\section{COMMUNICATION}

Dacorogna [2] and references cited within these two papers.

\section{Acknowledgments}

The author wishes to thank John Garnett for fruitful comments on an earlier draft of this manuscript. He is grateful to Wuchen Li and Yupeng Li for drawing the figures incorporated in the manuscript.

\section{References}

[1] G. Bouchitté, W. Gangbo, and P. Seppecher, Michell trusses and lines of principal actions, Math. Models Methods Appl. Sci. 18 (2008), no. 9, 1571-1603. MR 2446402

[2] B. DACOROGNA and W. GANGBO, Extension theorems for vector valued maps, J. Math. Pures Appl. (9), 85 (2006), no. 3, 313-344. MR 2210080

[3] A. G. MICHELL, The limits of economy of material in framedstructures, Phil. Mag. S. 68 (1904), 589-597.

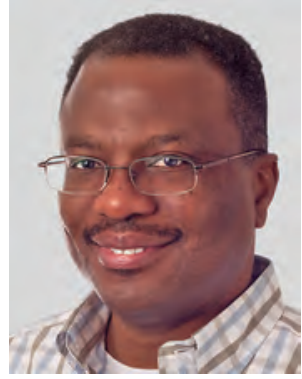

Wilfrid Gangbo

\section{ABOUT THE AUTHOR}

Wilfrid Gangbo's work in the calculus of variations and partial differential equations is funded by the National Science Foundation. He is dedicated to the promotion of mathematics in the Third World, especially in Africa, his continent of origin.

\section{Image Credits}

First photo of Dona Ana Bridge courtesy of Susan Reed at the English language Wikipedia [GFDL www . gnu . org/copyleft/fd] .htm 7), CC-BY-SA-3.0 (Ereativecommons .org/licenses/by $-\mathrm{sa} / 3.0 /$ ) or CC BY 2.5 (creativecommons.org/1icenses /by/2.5] ], via Wikimedia Commons.

Figures 1-4 and author photo courtesy of Wilfrid Gangbo.

Second photo of Dona Ana Bridge courtesy of Erik Cleves Kristensen (Flickr: Railway bridge over the Zambezi River) [CC BY 2.0 (creativecommons.org/1icenses/by/2.0)], via Wikimedia Commons.

\section{icerm.brown.edu}




\title{
THE GRADUATE STUDENT SECTION
}
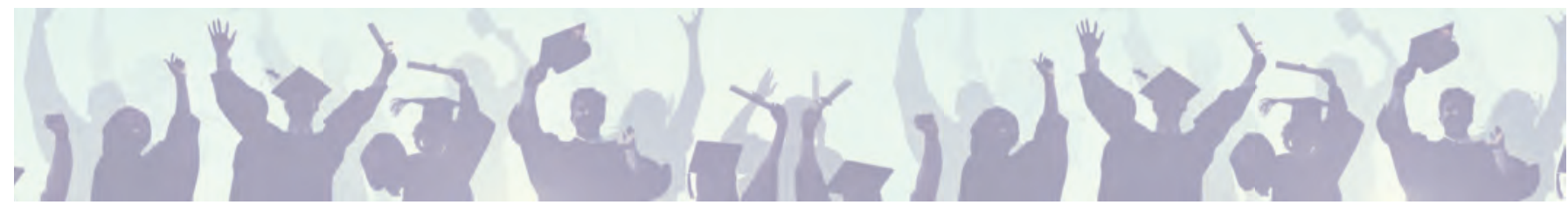

\section{Kathryn Hess Interview}

\author{
Conducted by Melinda Lanius
}

\author{
Communicated by Alexander Diaz-Lopez
}

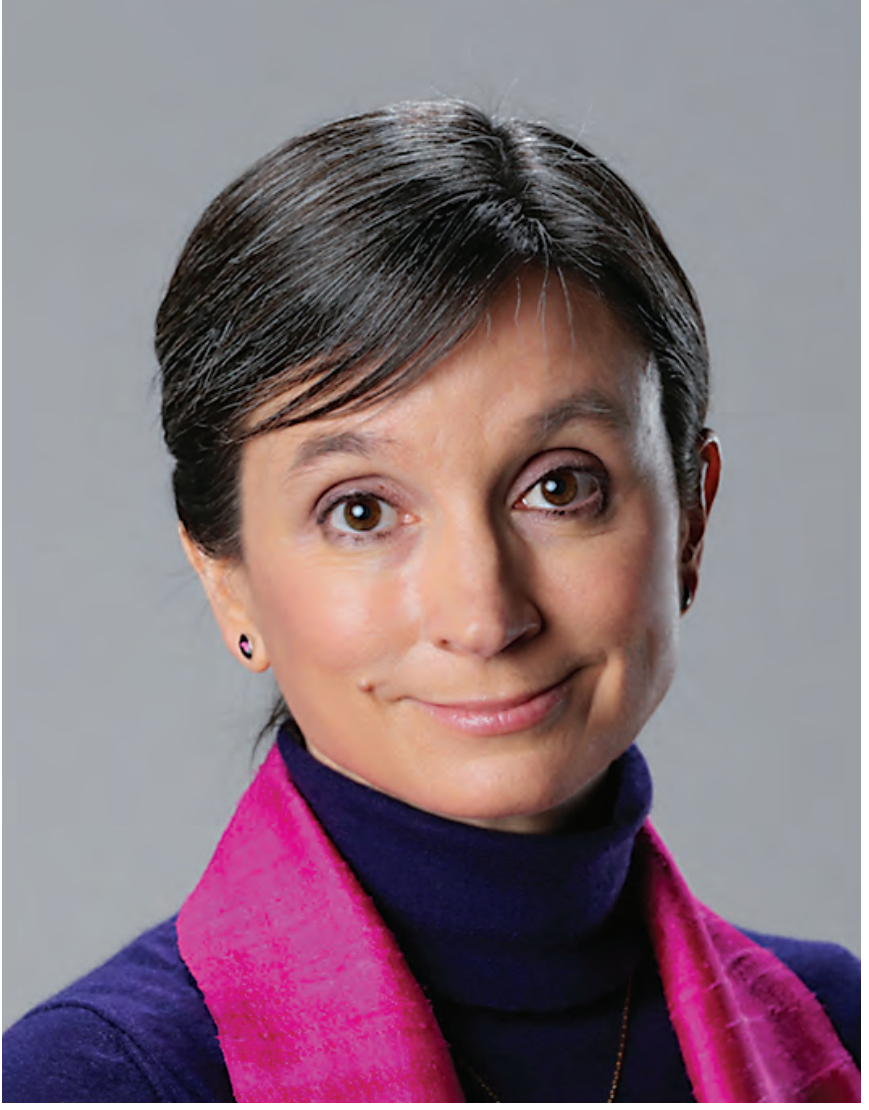

Kathryn Hess is associate professor of mathematics at École Polytechnique Fédérale de Lausanne. Her laboratory focuses on homotopy theory, category theory, algebraic topology, and their applications, primarily to the life sciences, in particular to neuroscience. Kathryn is a Fellow of the American Mathematical Society.

For permission to reprint this article, please contact: reprint-permission@ams.org.

DOI: http://dx.doi.org/10.1090/noti1646
Lanius: When and how did you know you wanted to be a mathematician?

Hess: Like many mathematicians, I started off thinking that I wanted to be a physicist. I fell in love with astronomy at the age of ten when I participated in an extracurricular astronomy course. I decided then that I wanted to be an astrophysicist, which was still my intention when I started my university studies. Of course, as a physics major I had to take many math courses, most of which I enjoyed very much, so that I was considering a double major in math and physics as I started my junior year. In the first semester of my junior year, I took a course in electricity and magnetism from the only woman math or physics professor I had during my studies, Bernice Durand. About halfway through the semester, she called me to her office and told me that, based on her observations, the only reason for my success in her course was that I was a very good mathematician, since I didn't seem to have much, if any, intuition for physics. I realized that she was rightin fact, it was almost a liberation to realize that I could focus on math instead of struggling to develop physical intuition-and I happily switched to being a math major, though I did still love astronomy.

Lanius: Who encouraged or inspired you?

Hess: My parents, first and foremost! They believed in my mathematical potential long before I did. Inspired by the fast-paced math courses founded by Julian Stanley at Johns Hopkins in the framework of the Study of Mathematically Precocious Youth, they created a similar program - the Mathematical Talent Development Project-in the town where we lived at the time, Eau Claire, Wisconsin, with the support of some of the math faculty there. I was a participant in the inaugural class of the program, which enabled me to get through all of precalculus high school 
math by the time I was 13 . Remarkably, of the 14 students in that inaugural class, at least two of us have gone on to become academic mathematicians at research universities.

While a student in that program, I heard a guest lecture from Dr. Karin Chess, a UW-Eau Claire mathematician, who provided my first inkling of what pure math might be. She spoke to us about non-Euclidean geometries, which I found absolutely fascinating: that one had the right to fiddle with the axioms of geometry, leading to such remarkable consequences for the overall structure!

Lanius: How would you describe your work to a graduate student?

Hess: My work in "pure" algebraic topology concerns homotopy theory. I'm particularly fascinated by what Waldhausen called "brave new algebra" (though he may have meant it in a somewhat more restricted sense): studying up-to-homotopy analogues and generalizations of classical results in algebra and algebraic geometry, such as the beautiful Galois correspondence.

Throughout my career I have worked on a number of side projects in applications of topology, such as applying knot theory to the study of polymers. This mathematical hobby started to play a central role in my research life a few years ago, when I began to collaborate more seriously and regularly with life scientists, particularly neuroscientists. Currently, together with the neuroscientists from the Blue Brain Project, we are applying algebraic topology to analyzing how the structure of the brain shapes its function, which is one of the central mysteries of neuroscience. The connections among the neurons in the brain form an incredibly rich and intricate structure, of which it is difficult to provide a quantitative global description. The spiking patterns of neurons in reaction to stimuli are, if anything, even more complex. Examining structure and activity through the filter of algebraic topology gives rise to quantitative descriptions of both and enables us to discern a reflection of the connectivity structure of a neural circuit in the shape of its response to stimuli. If we develop a deep understanding of what a normal pattern of response to a given input stimulus looks like, expressed in the language of algebraic topology, then we could perhaps use this knowledge to detect and quantify brain pathology, such as that arising in Alzheimer's or schizophrenia. Better comprehension of the brain's fantastically efficient information-processing structure could also probably be leveraged to improve artificial neural networks.

Lanius: What is a typical workday like?

Hess: It's easier for me to say what is involved in a typical workweek during the semester, since the structure of my days varies quite bit, which I appreciate. If I'm not traveling, I meet individually with each of my graduate students and with any project or masters' students I might have at least once a week. Teaching-preparation, classroom time, and related administrative work-usually fills the equivalent of a full day of work, more if I'm teaching a new course. I try to spend one day a week at the headquarters of the Blue Brain Project, to work with the members of my group who are there. We'll typically meet as a group for a brainstorming session, discussing the progress different team members have made over the past week and planning the next stages of each project on which we're collaborating. I'll also meet individually with various group members in order to discuss particular details of their projects in more depth.

Some time during the week-perhaps 20 percent on average-has to be devoted to various service tasks, either to the mathematical community or to my university: editorial work, refereeing, conference planning, writing letters of recommendation, committee work, etc.

When all is said and done, if I have the equivalent of one full day in the week for quiet thinking about research and reading articles, I feel lucky!

Lanius: What is the work culture like at École Polytechnique Fédérale de Lausanne and in the Laboratory for Topology and Neuroscience?

Hess: The team with which I work on applied topology projects is composed of members with varying backgrounds. Among my postdocs are researchers with PhDs in homotopy theory, knot theory, computational algebraic topology, and theoretical computer science. We collaborate closely with neuroscientists, cancer biologists, and material scientists, among others. It's a challenge learning to speak a common language, but once we do, the variety of our backgrounds enables us to progress rapidly, in directions in which none of us could have ventured alone.

I have complete freedom to choose the projects on which the team works and am always open to initiatives from team members. Many of our projects have been the result of pure serendipity, such as the time I ended up standing in a buffet line just behind a new colleague in material science. The informal chat that started as we waited in line led to a publication in Nature Communications.

One aspect of our applied work that I find very exciting is the way that it is leading to new theory as well, the development of new theoretical tools motivated by the applications we're studying.

Lanius: How do you balance career and outside interests?

Hess: The balance has shifted throughout my career, based on the number and ages of my children, as essentially all of my non-math time is devoted to family. As often as possible, family time involves hiking in the mountains, since we're lucky enough to live in Switzerland.

When the children were young, spending time with them was my highest priority. Once they were all teenagers or adults, I felt that I could let mathematics have an equal priority.

Vacation and relaxation time is important, of course, to avoid running out of energy or inspiration. A crucial lesson that I learned was that I couldn't force my brain to 
continue thinking about mathematics if it was sending me messages that it needed a break. It's more efficient to take a break-and go for a walk, read a book, or work in the garden-and then to return to work refreshed and relaxed.

Lanius: Are there any speed bumps in your journey that you could share with us?

Hess: I was clearly less productive as a researcher while I had young children, which I think is probably almost inevitable. The academic world is often not very understanding about women - or, more generally, parents-who have less time for research when their children are young. It helped me that there were a few senior colleagues who continued to believe in me as a researcher and told me so, even in the years when I was less productive.

Lanius: What advice do you have for graduate students?

Hess: Learn to give good talks! Many colleagues' first impression of you will come from hearing you speak. If you succeed in communicating your passion for your work, then more people will be interested in what you're doing.

\section{Learn to give good talks!}

If there's no formal framework in which to practice giving talks, set one up with the other graduate students at your university. Remember that constructive criticism from your peers is an important part of such a learning process. Ask your professors if they can recommend any videos of particularly good talks that you can watch for inspiration.

Lanius: If you could recommend one article to graduate students, what would it be?

Hess: I suggest that graduate students ask their advisors for two or three examples of beautifully written articles in their field, even if the subject is not directly related to their thesis problem. In order to learn to write well, it's important to be exposed to exemplary writing.

Lanius: Any final comments or advice?

Hess: Embrace serendipity! It may lead you to an exciting, unexpected destination.

\section{Photo Credits}

Interviewee photo (c) Alain Herzog/EPFL.

Interviewer photo of courtesy of Melinda Lanius.

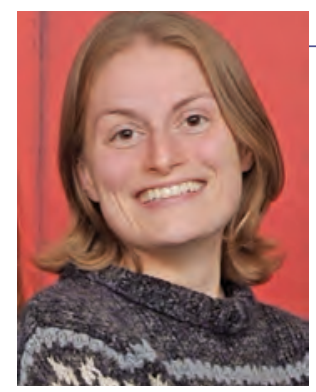

\section{ABOUT THE INTERVIEWER}

Melinda Lanius, a Wellesley College graduate, is currently earning her $\mathrm{PhD}$ in mathematics at the University of Illinois at Urbana-Champaign.
FEATURED TITLES FROM THE

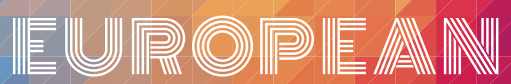

IIIM

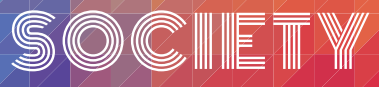

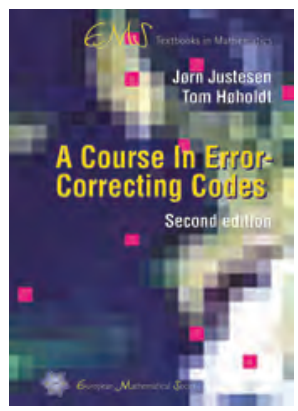

A Course in ErrorCorrecting Codes

Second Edition

Jørn Justesen, Technical

University of Denmark, Lyngby, and Tom Høholdt, Technical University of Denmark, Lyngby

This book, updated and enlarged for the second edition, is written as a text for a course aimed at third- or fourth-year graduate students in discrete mathematics, computer science, or communication engineering and is also a suitable introduction to coding theory for researchers from related fields.

EMS Textbooks in Mathematics, Volume 20; 2017; 226 pages; Hardcover; ISBN: 978-3-03719-179-8; List US\$45; AMS members US\$36; Order code EMSTEXT/20

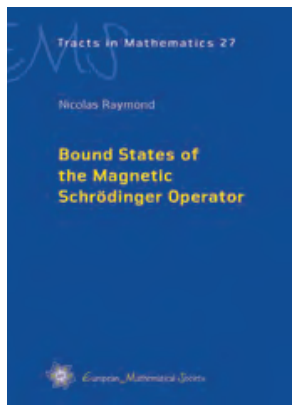

\section{Bound States of the Magnetic Schrödinger Operator}

Nicolas Raymond, Université de Rennes, France

This book is a synthesis of recent advances in the spectral theory of the magnetic Schrödinger operator. It can be considered a catalog of concrete examples of magnetic spectral asymptotics.

EMS Tracts in Mathematics, Volume 27; 2017; 394 pages; Hardcover; ISBN: 978-3-03719-169-9; List US\$78; AMS members US\$62.40; Order code EMSTM/27

\section{Explore more titles at bookstore.ams.org.}

Publications of the European Mathematical Society (EMS). Distributed within the Americas by the American Mathematical Society.

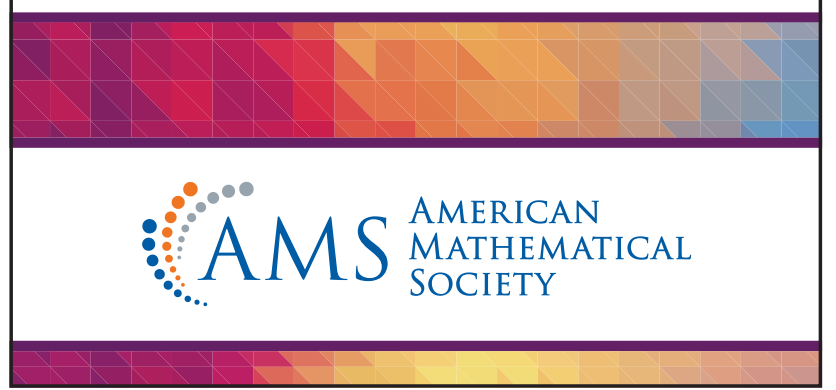



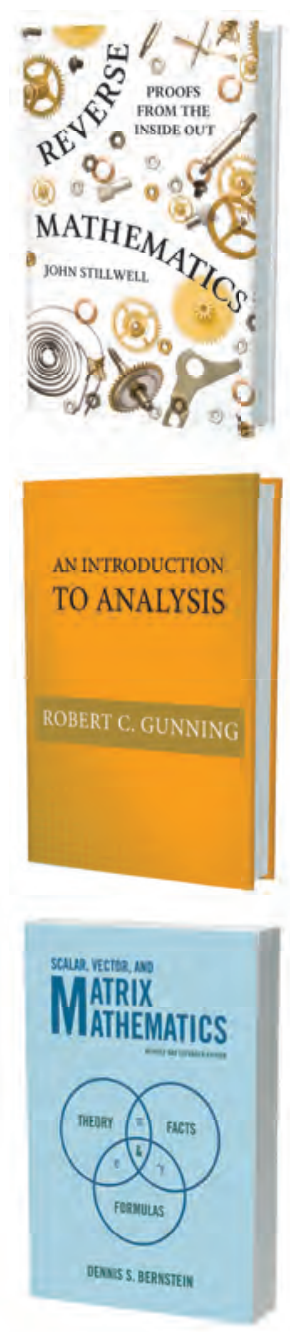

\section{Scalar, Vector, and Matrix Mathematics}

Theory, Facts, and Formulas

Revised and Expanded Edition

Dennis S. Bernstein

Paper $\$ 99.50$

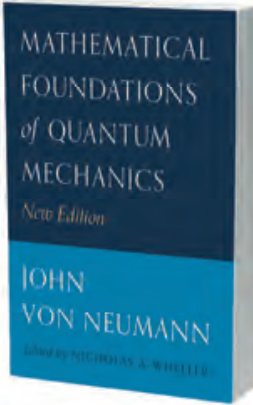

\section{Mathematical Foundations of Quantum Mechanics}

\section{New Edition}

John von Neumann

Translated by Robert T. Beyer

Edited by Nicholas A. Wheeler

Paper $\$ 99.50$

\section{The Mathematics of Various Entertaining Subjects}

Research in Games, Graphs, Counting, and Complexity, Volume 2

Edited by Jennifer Beineke \&

Jason Rosenhouse
"Reverse mathematics is a major research direction in the foundations of mathematics and mathematical logic, and the insights obtained from reverse mathematics will interest a wide mathematically minded audience. Stillwell's book is self-contained and includes much background material in analysis, mathematical logic, combinatorics, and computability. I heartily commend this very readable and accessible book."

—Stephen Simpson, Vanderbilt University

"Gunning's book is a great introduction to analysis that presents precisely what an honors analysis course should include. The writing is rigorous but lively, and much interesting mathematics is packed in it." —Wilhelm Schlag, University of Chicago
"This is a book that any mathematician, physicist, or engineer would want to have at hand. If you are looking for a particular mathematical identity, an inequality, or a fact about matrices, then it is most likely that you will find it in this encyclopedic work." —Omran Kouba, Higher Institute for Applied Sciences and Technology, Damascus
In this new edition of this classic work, mathematical physicist Nicholas Wheeler has completely reset the book in TeX, making the text and equations far easier to read. He has also corrected a handful of typographic errors, revised some sentences for clarity and readability, provided an index for the first time, and added prefatory remarks drawn from the writings of Léon Van Hove and Freeman Dyson. The result brings new life to an essential work in theoretical physics and mathematics.
"This book is a fascinating treasure trove of puzzles, brain teasers, and mathematical recreations that will keep your mind busy for months, if not years. A true gem that is destined to become a classic."

-Eli Maor, author of e: The Story of a Number

Published in association with the National Museum of Mathematics 


\section{THE GRADUATE STUDENT SECTION}

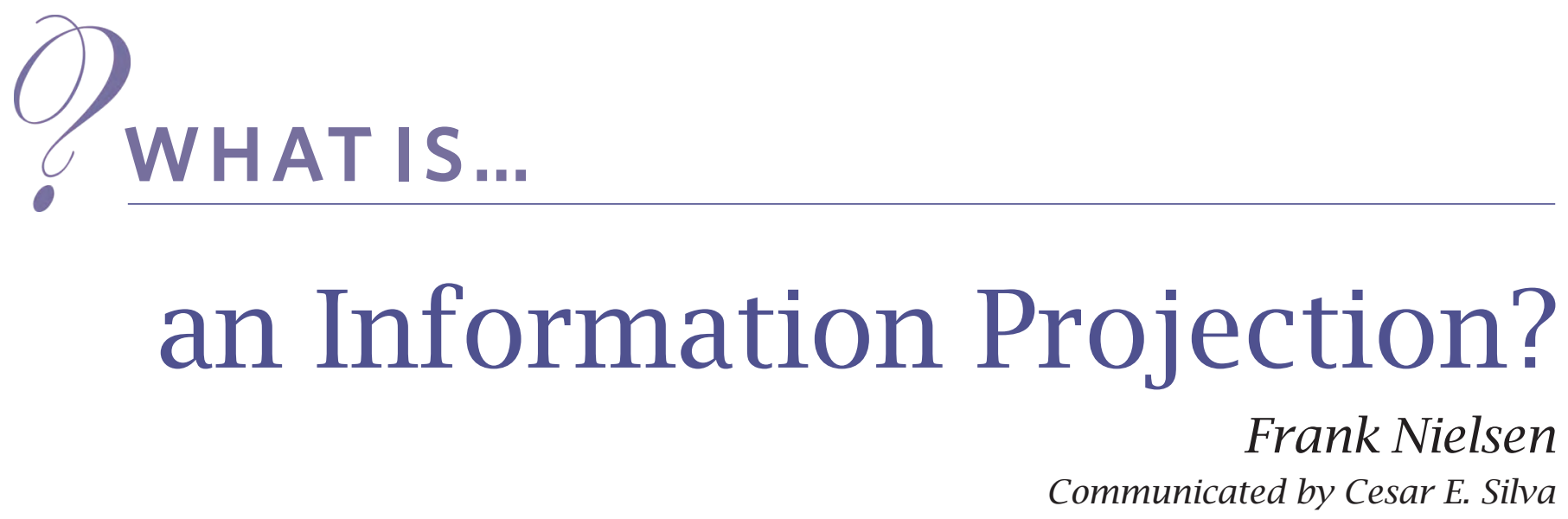

\section{Orthogonal Projections as Distance Minimizers}

In Euclidean geometry, the orthogonal projection $p_{S}$ of a vector $p$ onto a subset $S$ as in Figure 1 can be defined as the point(s) $q$ of $S$ minimizing the distance $D(p, q)$ from $p$ to $q$. In general, the projection may not be unique: for example, projecting the center of a unit ball onto its boundary sphere yields the full boundary sphere. However, the projection $p_{S}$ is always guaranteed to be unique when $S$ is an affine subspace.

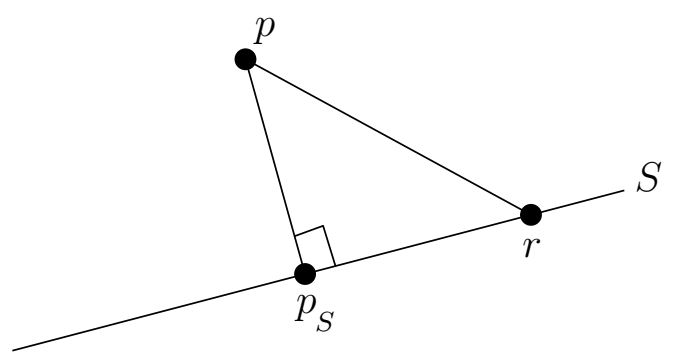

Figure 1. In Euclidean geometry, the orthogonal projection $p_{S}$ of $p$ onto $S$ can be defined as the minimizer of the Euclidean distance: it is unique when $S$ is affine.

We shall describe how these notions generalize to the dual geometry of information spaces.

\section{Dual Divergences and Information Projections}

In information theory [2], we prefer to use a dissimilarity measure $D(p, q)$ between probability distributions $p(x)$ and $q(x)$ instead of the Euclidean distance. Since those distortion measures are often asymmetric, $D(p, q) \neq D(q, p)$,

Frank Nielsen is professor of computer science at École Polytechnique, France, and senior researcher at Sony Computer Science Laboratories, Japan. His email address is Frank. Nielsen@acm .org.

For permission to reprint this article, please contact:

reprint-permission@ams.org.

DOI: http://dx.doi.org/10.1090/noti1647 we use the notation $D(p: q)$ to highlight the asymmetric property of information distances and call $D(p: q)$ a divergence, assumed to be infinitely differentiable.

Here the word "divergence" is not to be confused with the divergence operator from calculus. Similar to the Euclidean case, an information projection of $p \in M$ onto $S \subset M$ can be defined by minimizing the divergence $D(q: p)$ for $q \in S$. Since the divergence is asymmetric, we define a dual divergence $D^{*}(p: q)=D(q: p)$.

Information Monotonicity, Invariant Divergence, and Invariant Metric

For example, consider the space $M$ of Gaussian distributions on $\mathcal{X}$ the real line with

$$
p(x ; \xi)=p(x ; \mu, \sigma)=\frac{1}{\sqrt{2 \pi} \sigma} \exp \left(-\frac{(x-\mu)^{2}}{2 \sigma^{2}}\right)
$$

parameterized by $\xi=(\mu, \sigma)$. There exist many statistical distances $D\left(p\left(x ; \xi_{1}\right): p\left(x ; \xi_{2}\right)\right)$ for measuring the distortion between any two distributions of the statistical manifold $M$. However, assume we apply a mapping $y=k(x)$ (not necessarily one-to-one) and define the distributions

$$
p^{\prime}(y ; \xi)=\int_{\{x: k(x)=y\}} p(x ; \xi) \mathrm{d} x .
$$

Then we would like to have

$$
D\left(p^{\prime}\left(y ; \xi_{1}\right): p^{\prime}\left(y ; \xi_{2}\right)\right) \leq D\left(p\left(x ; \xi_{1}\right), p\left(x ; \xi_{2}\right)\right),
$$

with equality when $y=k(x)$ is one-to-one or when $k(x)$ is a sufficient statistic. This property of divergences is called the information monotonicity. Members of the class of statistical $f$-divergences

$$
I_{f}(p: q)=\int_{x} p(x) f(q(x) / p(x)) \mathrm{d} x
$$

defined for a convex function $f(u)$ satisfying $f(1)=0$ have this property and are called invariant divergences. These include all divergences represented as sums or integrals of elementary scalar divergences that satisfy the information monotonicity (except for divergences on binary alphabets, with $\chi=\{0,1\})$. Since $I_{g}(p: q)=$ 


\section{THE GRADUATE STUDENT SECTION}

$I_{f}(p: q)$ for $g(u)=f(u)+c(u-1)$ with $c \in \mathbb{R}$, we may assume that $f^{\prime}(1)=0$. Furthermore, since $I_{\lambda f}(p: q)=$ $\lambda I_{f}(p: q)$ for $\lambda>0$, we define the standard $f$-divergences for $f^{\prime \prime}(1)=1$. The dual $f$-divergence $I_{f}^{*}(p: q)=I_{f}(q: p)$ of a standard $f$-divergence $I_{f}(p: q)$ is a standard $f$ divergence obtained for the convex generator $f^{\diamond}(u)=$ $u f(1 / u)$.

Any standard $f$-divergence induces a Riemannian geometry $(M, g)$ given by a certain "Fisher information matrix." This metric is called the Fisher metric and allows one to define the Fisher orthogonality of vectors.

\section{Dual Geodesic Projections and Dual Pythagorean Theorems}

The most fundamental distance or divergence in information theory is the Kullback-Leibler invariant divergence, commonly called $I$-divergence for short,

$$
I(p: q)=I_{f}(p: q)=\int_{\chi} p(x) \log \frac{p(x)}{q(x)} \mathrm{d} x,
$$

obtained for $f(u)=-\log u$.

Recall that in Euclidean geometry the line segment $\left[p p_{S}\right]$ meets the subset $S$ orthogonally at the projected point $p_{S}$ (that is, $\left[p p_{S}\right] \perp S$ ) and that the projection $p_{S}$ is guaranteed to be unique when $S$ is affine. Information geometry extends these results by revealing the dual nature of the $I$-divergence geometry using the framework of differential geometry. Consider $M$ as a smooth manifold of a family of distributions. When the family belongs to the exponential families (e.g., Gaussian distributions), the density can be written canonically as

$$
p(x ; \theta)=\exp (\langle\theta, t(x)\rangle-F(\theta)),
$$

where $\langle\cdot, \cdot\rangle$ denotes the Euclidean inner product, $F(\theta)=$ $\log \int_{\chi} \exp (\langle\theta, t(x)\rangle) \mathrm{d} x$ is a $C^{\infty}$ (convex) "Legendre" function that ensures normalization to a probability distribution, and $\theta$ is the natural parameter belonging to the parameter space $\Theta=\left\{\theta: \int_{x} \exp (\langle\theta, t(x)\rangle) \mathrm{d} x<\infty\right\}$. Any Legendre function $F(\theta)$ has a conjugate Legendre function [1] $F^{\star}(\eta)$ defined by

$$
F^{\star}(\eta)=\sup _{\theta \in \Theta}\{\langle\theta, \eta\rangle-F(\theta)\} .
$$

The parameter $\eta=\eta(\theta)$ is called the expectation parameter since $E_{x \sim p(x ; \theta)}[t(x)]=\eta$. For the univariate Gaussian family, we get $\theta=\left(\frac{\mu}{\sigma^{2}},-\frac{1}{2 \sigma^{2}}\right)$ and $\eta=\left(\mu, \mu^{2}+\sigma^{2}\right)$ (with $\left.t(x)=\left(x, x^{2}\right)\right)$.

Thus a distribution of an exponential family can be indexed by either its natural parameter $\theta$ or its expectation parameter $\eta: p(x ; \theta)=p(x ; \eta)$, with the conversion $\theta=$ $\nabla F^{\star}(\eta)$ and $\eta=\nabla F(\theta)$, where $\nabla$ denotes the gradient operator.

It turns out that the $I$-divergence between two distributions of the same exponential family is equivalent to a Bregman divergence:

$$
I\left(p\left(x ; \theta_{1}\right): p\left(x ; \theta_{2}\right)\right)=B_{F}\left(\theta_{2}: \theta_{1}\right),
$$

where

$$
B_{F}\left(\theta_{2}: \theta_{1}\right)=F\left(\theta_{2}\right)-F\left(\theta_{1}\right)-\left\langle\theta_{2}-\theta_{1}, \nabla F\left(\theta_{1}\right)\right\rangle .
$$

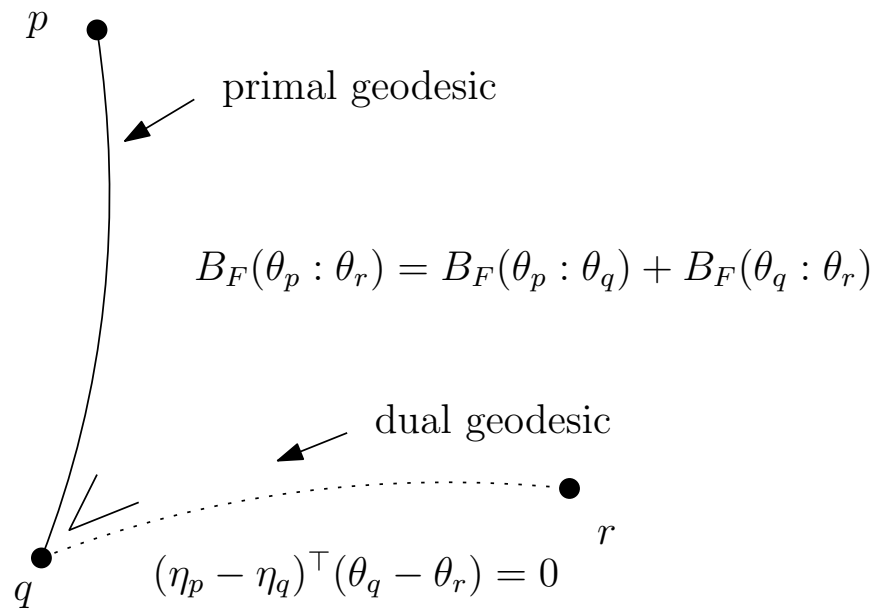

Figure 2. The Pythagorean theorem in an information space.

We can also express the information divergence using the $\eta$-parameters as $I\left(p\left(x ; \eta_{1}\right): p\left(x ; \eta_{2}\right)\right)=B_{F^{\star}}\left(\eta_{1}: \eta_{2}\right)$.

To connect two distributions $p\left(x ; \theta_{1}\right)$ and $p\left(x ; \theta_{2}\right)$ on the exponential family manifold $M=\{p(x, \theta): \theta \in \Theta\}$, we may consider the path $\gamma_{e}\left(\theta_{1}, \theta_{2}, a\right)=p(x ; \theta(a))$ with $\theta(a)=(1-a) \theta_{1}+a \theta_{2}$ for $a \in[0,1]$. This path forms a $1 \mathrm{D}$ exponential family, and we can rewrite it by taking the logarithm as

$\log p(x, \theta(a))=(1-a) \log p\left(x ; \theta_{1}\right)+a \log p\left(x ; \theta_{2}\right)-F(\theta(a))$.

This is a linear interpolation on the logarithmic scale, hence its name $e$-geodesic $\gamma_{e}\left(\theta_{1}, \theta_{2}\right)=\left\{\gamma_{e}\left(\theta_{1}, \theta_{2}, a\right)\right.$ : $a \in[0,1]\}$, which stands for exponential geodesic. Or, we can alternatively connect the two distributions using the path $\gamma_{m}\left(\eta_{1}, \eta_{2}, a\right)=p(x ; \eta(a))=p\left(x ;(1-a) \eta_{1}+\right.$ $\left.a \eta_{2}\right)$. For discrete probability distributions $p_{1}$ and $p_{2}$ of the probability simplex, we get the mixture distribution $p(a)=(1-a) p_{1}+a p_{2}$, hence its name $m$-geodesic $\gamma_{m}\left(\eta_{1}, \eta_{2}\right)=\left\{\gamma_{m}\left(\eta_{1}, \eta_{2}, a\right): a \in[0,1]\right\}$, which stands for mixture geodesic. The $e$-geodesic and $m$-geodesic are visualized as straight line segments in the $\theta$ - and $\eta$-coordinate systems, respectively. Let us define an $e$-flat subspace ( $e$-flat for short) as an affine subspace in the $\theta$-coordinate system and an $m$-flat subspace ( $m$-flat for short) as an affine subspace in the $\eta$-coordinate system.

The $e$-projection $p_{S}^{e}$ of $p$ onto $S$ is defined by minimizing $I(q: p)$ for $q \in S$ and is unique when $S$ is $m$-flat. The $m$-projection $p_{S}^{m}$ of $p$ onto $S$ is defined by minimizing $I(p: q)$ for $q \in S$ and is unique when $S$ is $e$-flat.

Similar to the Euclidean case, the proof of the uniqueness of the dual information geodesic projections follows from the dual Pythagorean theorems of Bregman divergences (Figure 2): When the triangle $p q r$ is such that $\gamma_{m}\left(\eta_{p}, \eta_{q}\right) \perp \gamma_{e}\left(\theta_{q}, \theta_{r}\right)$ (dual geodesics perpendicular at $q)$, we have

$$
B_{F}\left(\theta_{p}: \theta_{r}\right)=B_{F}\left(\theta_{p}: \theta_{q}\right)+B_{F}\left(\theta_{q}: \theta_{r}\right) .
$$

The orthogonality implies that $\left(\eta_{p}-\eta_{q}\right)^{\top}\left(\theta_{q}-\theta_{r}\right)=0$. 


\section{THE GRADUATE STUDENT SECTION}

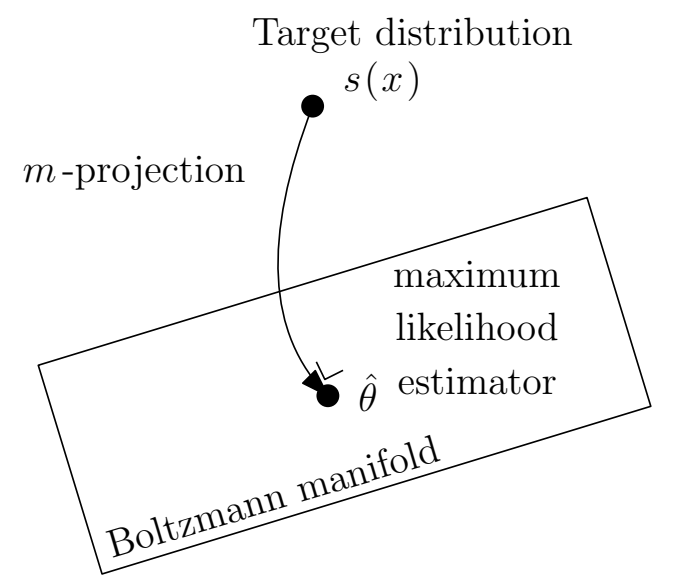

Figure 3. The maximum likelihood estimator $\hat{\theta}$ of a Boltzmann machine is the unique $m$-projection of the target distribution $s(x)$ onto the Boltzmann manifold.

When the triangle $p q r$ is such that $\gamma_{e}\left(\theta_{p}, \theta_{q}\right) \perp$ $\gamma_{m}\left(\eta_{q}, \eta_{r}\right)$ (dual geodesics perpendicular at $q$ ), we have

$$
B_{F^{\star}}\left(\eta_{p}: \eta_{r}\right)=B_{F^{\star}}\left(\eta_{p}: \eta_{q}\right)+B_{F^{\star}}\left(\eta_{q}: \eta_{r}\right) \text {. }
$$

The orthogonality implies that $\left(\theta_{p}-\theta_{q}\right)^{\top}\left(\eta_{q}-\eta_{r}\right)=0$.

To illustrate the geodesic information projections, let us consider the following two examples: In machine learning [3], a Boltzmann machine is a fully interconnected network of $n$ stochastic units that defines an exponential family distribution on $X=\{0,1\}^{n}$ by

$$
p(x ; \theta)=\exp \left(\sum_{i} \theta_{i} x_{i}+\sum_{i<j} \theta_{i j} x_{i} x_{j}-F(\theta)\right),
$$

where the $\theta_{i j}$ 's are the weights connecting unit $i$ to unit $j$ and the $\theta_{i}$ 's are the bias parameters. Boltzmann machines are universal approximators: they can represent any smooth distribution within any prescribed accuracy. The set of all machines $M=\{p(x ; \theta): \theta \in \Theta\}$ defines the Boltzmann $e$-flat manifold. Given a target distribution $s(x)$ that we wish to learn from, the Maximum Likelihood Estimator (MLE) $\hat{\theta}$ is characterized by the unique $m$ projection of $s(x)$ onto $M$ as in Figure 3.

The Maximum Entropy (MaxEnt) principle yields a distribution $p(x)$ maximizing the Shannon entropy under a set of $D$ moment constraints $E_{X}\left[t_{i}(X)\right]=m_{i}, i \in$ $\{1, \ldots, D\}$. It can be shown that the MaxEnt distribution belongs to an exponential family and is the unique $e$-projection of the uniform distribution on the $m$-flat manifold $\left\{X: E\left[t_{1}(X)\right]=m_{1}, \ldots, E_{X}\left[t_{D}(X)\right]=m_{D}\right\}$.

A geodesic information projection $\min _{q \in S} D(q: p)$ can be rewritten as a point-set divergence $D(S: p)$. Consider two submanifolds $U$ and $V$ of $S$, and define

$$
D(U: V)=\min _{u \in U, v \in V} D(u: v)=D\left(u^{*}, v^{*}\right),
$$

where $u^{*}$ and $v^{*}$ form a closest pair between $U$ and $V$. We approximate a closest pair between the submanifolds by the alternating minimization algorithm: Begin with $v_{1} \in V$, minimize $D\left(u: v_{1}\right)$ by an information projection to get $u_{1}$, and minimize $D\left(u_{1}: V\right)$ to get $v_{2}$ by a dual information

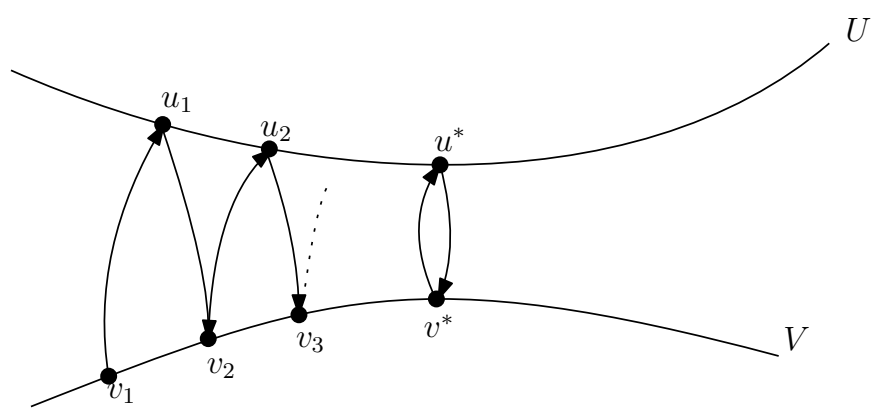

Figure 4. The alternating geodesic projection algorithm for computing the divergence between submanifolds always converges.

projection, etc. This alternating projection algorithm always converges, since the divergence decreases and is lower bounded by 0 . Moreover, there is a unique closest pair when $V$ is flat and $U$ is dually flat as in Figure 4.

\section{Dual Geometry of Information Projections}

Information projections are a core concept of information sciences that are met whenever minimizing divergences [2]. Depending on whether the minimization is carried out on the left argument of the divergence $D(\cdot: \cdot)$ or on its right argument (that is, the left argument of the dual divergence $D^{*}$ ), we end up with an information projection or a dual information projection. The geometric nature of information projections is elucidated using the dual geodesics of information geometry. In differential geometry, the notion of a geodesic $\gamma(p, q)$ passing through two points $p, q \in M$ depends on a connection. A connection $\prod_{p \rightarrow q}$ indicates how to transport vectors from one tangent plane $T_{p}$ to any other tangent plane $T_{q}$. A geodesic is then defined as an auto-parallel curve satisfying $\nabla_{\dot{\gamma}} \dot{\gamma}=0$, where $\nabla$ is the covariant derivative associated to the connection. An affine connection $\nabla$ is defined by its Christoffel symbols. The fundamental structure of information geometry is a pair of torsionfree affine connections $\nabla$ and $\nabla^{*}$ that are coupled to a Riemannian metric tensor $g$ with $g^{*}=g$ and $\frac{\nabla+\nabla^{*}}{2}=\nabla^{g}$, the Levi-Civita metric connection. This dualistic structure $\left(M, g, \nabla, \nabla^{*}\right)$ can be built from any divergence $D(\cdot: \cdot)$ and generalizes Euclidean geometry. A manifold is called $\nabla$-flat if the Christoffel coefficients of $\nabla$ vanish in some coordinate system.

A dually flat geometry can be built from any smooth strictly convex function via the Legendre transformation and the corresponding dual Bregman divergences: those geometries are said to be dually flat because their primal and dual geodesics can be expressed as straight lines in the primal and dual affine coordinate systems, respectively.

Bregman divergences are the canonical divergences of dually flat manifolds: any dually flat manifold is induced by a corresponding Bregman divergence. Many stochastic neuronal network models (like the stochastic multilayer perceptrons [3] or the Boltzmann machines) handle exponential families in disguise and can thus be 


\section{THE GRADUATE STUDENT SECTION}

studied using the method of information geometry and its dual information projections.

\section{References}

[1] Heinz H. BauschKe and Yves Lucet, What is a Fenchel conjugate? Notices Amer. Math. Soc. 59 (2012), no. 1, 44-46. MR 2908159

[2] Thomas M. Cover and Joy A. Thomas, Elements of Information Theory, Second edition, John Wiley \& Sons, 2006. MR 2239987

[3] IAN Goodfellow, Yoshua Bengio, and Aaron Courville, Deep Learning, MIT Press, 2016. MR 3617773

\section{Image Credits}

Figures 1-4 courtesy of Frank Nielsen.

Photo of Frank Nielsen courtesy of Maryse Beaumont.

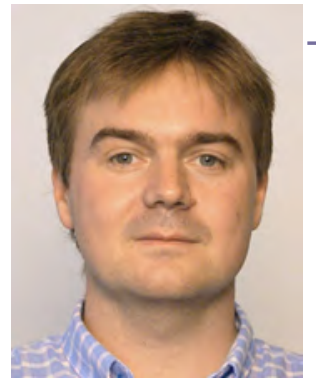

\section{ABOUT THE AUTHOR}

When not sitting on a transcontinental airplane, Frank Nielsen enjoys walking along the coastal paths of the French Riviera.

$$
\begin{aligned}
& \text { 香港中文大學 } \\
& \text { TheChinese University of Hong Kong }
\end{aligned}
$$

Applications are invited for:-

\section{Department of Mathematics}

\section{Research Assistant Professors}

(Ref. 170002LV) (Closing date: June 30, 2018)

Founded in 1963, The Chinese University of Hong Kong (CUHK) is a forward-looking comprehensive research university with a global vision and a mission to combine tradition with modernity, and to bring together China and the West.

The Department of Mathematics in CUHK has developed a strong reputation in teaching and research. Many faculty members are internationally renowned and are recipients of prestigious aw ards and honours. The graduates are successful in both academia and industry. The Department is highly ranked internationally. According to the latest rankings, the Department is 51st-75th in the Academic Ranking of World Universities, 36th in the QS World University Rankings and 34th in the US News Rankings.

The Department is now inviting applications for the position of Research Assistant Professor in all areas of mathematics. Applicants should have a relevant $\mathrm{PhD}$ degree and good potential for research and teaching.

Appointments will initially be made on contract basis for up to three years commencing August 2018, renewable subject to mutual agreement.

Applications will be considered on a continuing basis but candidates are encouraged to apply by March 31, 2018.

Application Procedure

The University only accepts and considers applications submitted online for the posts above. For more information and to apply online, please visit http://career.cuhk.edu.hk

Frank Nielsen

\section{From the January 2018 Electronic Newsletter of the International Mathematical Union ${ }^{1}$}

The Committee for Women in Mathematics funded 10 proposals, most devoted to developing regional networks for Women in Mathematics, in Africa, Latin America, and Asia. Often the initiatives take the form of a meeting with both a mathematical part and a career development part. This is the case for two regional meetings of the African Women in Mathematics Association, one in Addis Ababa (Ethiopia) for East Africa and one in Ibadan (Nigeria) for West Africa, and also for the second Central Asia Women in Mathematics Association meeting in Uzbekistan. There are other meetings in India, Macedonia, El Salvador, and Uruguay. The African Women in Mathematics Association will also be writing portraits of African women mathematicians. Two further events are taking place in Europe, an ICTP school in Trieste (Italy) on Dynamical Systems, with all female organizers and lecturers, and the European Women in Mathematics General Meeting in Graz (Austria). In both cases the grant will be used to support the attendance of women from developing countries. The remaining part of the budget will be used to support $(\mathrm{WM})^{2}$, the first World Meeting for Women in Mathematics taking place on 31 July 2018 as a satellite event of ICM Rio. ${ }^{2}$ In particular, women from all over the world who are supported by the Open Arms program have been invited to attend (WM) ${ }^{2}$, with no registration fee. Submissions of scientific mathematical posters and thematic posters on women in mathematics to $(\mathrm{WM})^{2}$ is possible until 30 March. 


\section{Start an}

AMS Graduate Student Chapter at your institution
AMS Graduate Student Chapters are designed to generate interest in the mathematical sciences and encourage students in their mathematical pursuits by providing them with new opportunities and experiences.

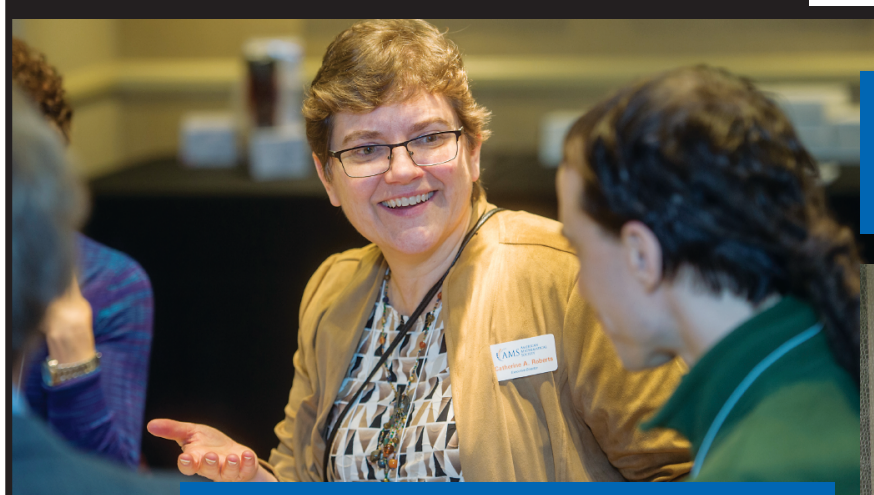

Network and discover career opportunities

Find opportunities for community outreach

Collaborate with fellow students \& faculty
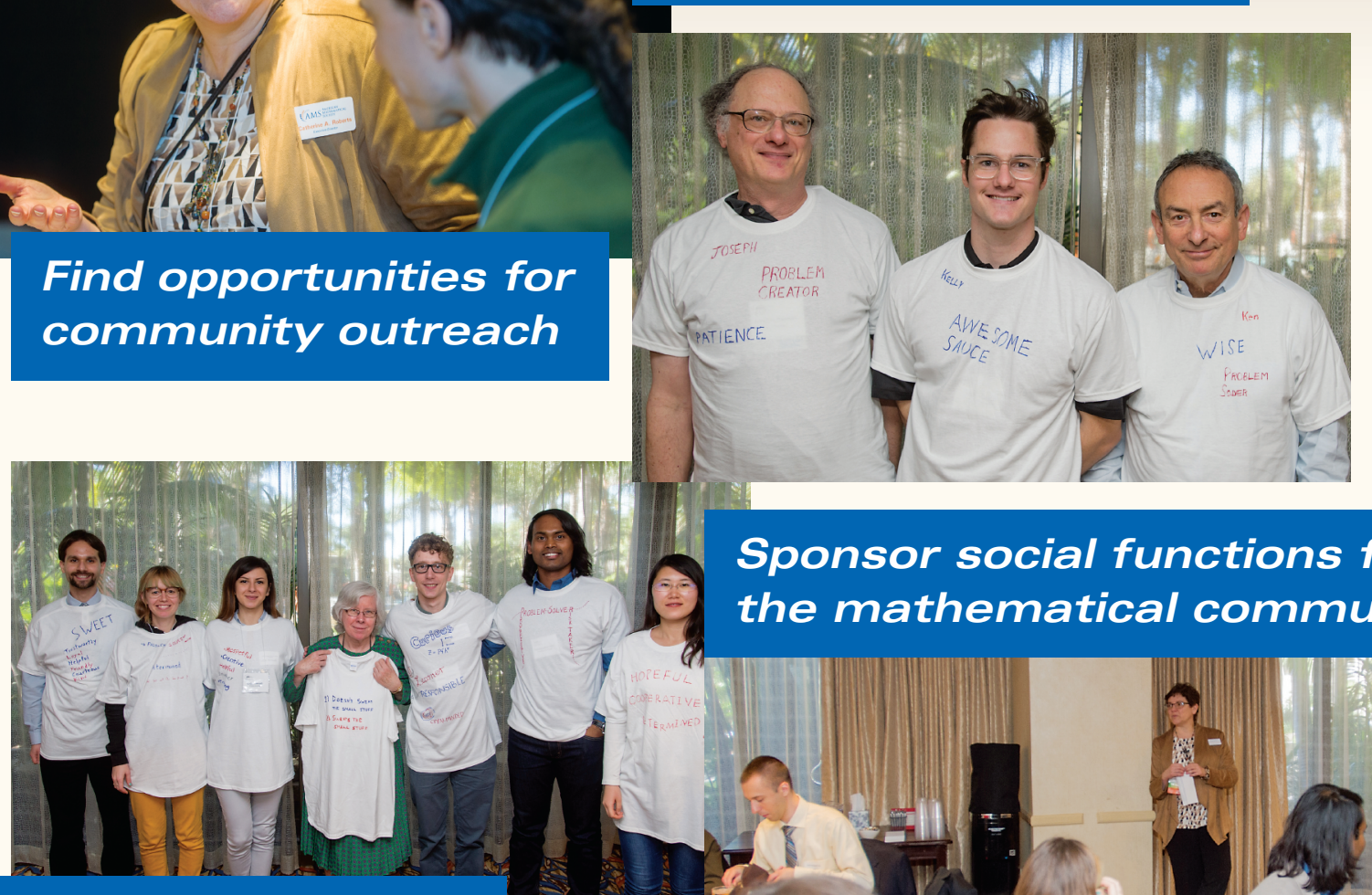

Sponsor social functions for the mathematical community

The AMS provides up to $\$ 500$ annually for mathematical activities. Visit the "Chapter in a Box" at www.ams.org/studentchapters to get started on your petition!

\section{Suggested deadline to submit petition: October $1^{\text {st }}$}




\title{
Gender Differences in First Jobs for New US PhDs in the Mathematical Sciences
}

\author{
by Marie A. Vitulli
}

\section{Communicated by Harriet Pollatsek}

ABSTRACT. Among all US-earned PhDs in mathematics, women have slightly lower initial unemployment rates than men, but their first post-PhD jobs are less likely than men's to be at top universities and in business or industry, despite the prestige of their PhD-granting department. Among non-US-citizens, women have higher unemployment rates during 2001-2015. The percentage of women new PhDs who were employed at bachelor's-only departments was much higher than for men, with the greatest difference occurring in 2012-2015.

We take a long-term look at employment trends for new doctorates with an eye towards gender, citizenship, and gender $\times$ citizenship $^{1}$ differences by analyzing data from the 1991-2015 AMS-ASA-IMS-MAA-SIAM Annual Surveys of the Mathematical Sciences [1].2

We will see that the same differences that Flahive and the current author observed in previous studies in 1997 and 2010 [3] continue today, namely that women were initially employed at academic institutions whose highest degree in mathematics is a bachelor's degree at a substantially higher rate than men, and men were initially employed in business and industry at a considerably higher rate than women.

From 1991-2015 women received about 29\% of the mathematics doctorates from US departments, a bit higher more recently for non-US citizens, as shown in Table 1. During this period US citizens received $46 \%$ of the mathematics doctorates, 29\% of these to women. (In Table 1 the PhDs whose citizenship was unknown at the time of the surveys appear in the All PhDs column but do not appear in the US or Non-US columns.) The percentage of new women PhDs was lowest during 1991-2000, when the rate for US citizens was higher than for non-citizens; the opposite held for men.

Marie A. Vitulli is professor emerita at the University of Oregon. Her email address is vitu11i@uoregon. edu.

For permission to reprint this article, please contact: reprint-permission@ams.org.

DOI: http://dx.doi.org/10.1090/noti1649
Table 1. PhDs Granted (Percentages of all PhDs in Two Column Citizenship Group) by Citizenship and Gender

\begin{tabular}{|c|c|c|c|c|c|c|}
\hline \multirow[b]{2}{*}{ Period } & \multicolumn{2}{|c|}{ US } & \multicolumn{2}{|c|}{ Non-US } & \multicolumn{2}{|c|}{ All PhDs } \\
\hline & $\mathbf{F}$ & $\mathbf{M}$ & $\mathbf{F}$ & $\mathbf{M}$ & $\mathbf{F}$ & $\mathbf{M}$ \\
\hline $\begin{array}{c}1991- \\
2000\end{array}$ & $\begin{array}{l}1350 \\
(27 \%)\end{array}$ & $\begin{array}{l}3622 \\
(73 \%)\end{array}$ & $\begin{array}{l}1147 \\
(21 \%)\end{array}$ & $\begin{array}{l}4293 \\
(79 \%)\end{array}$ & $\begin{array}{l}2597 \\
(24 \%)\end{array}$ & $\begin{array}{l}8291 \\
(76 \%)\end{array}$ \\
\hline $\begin{array}{c}2001- \\
2011\end{array}$ & $\begin{array}{l}1950 \\
(30 \%)\end{array}$ & $\begin{array}{l}4531 \\
(70 \%)\end{array}$ & $\begin{array}{l}2501 \\
(33 \%)\end{array}$ & $\begin{array}{l}5187 \\
(67 \%)\end{array}$ & $\begin{array}{l}4468 \\
(31 \%)\end{array}$ & $\begin{array}{l}9761 \\
(69 \%)\end{array}$ \\
\hline $\begin{array}{c}2012- \\
2015\end{array}$ & $\begin{array}{c}972 \\
(28 \%)\end{array}$ & $\begin{array}{l}2548 \\
(72 \%)\end{array}$ & $\begin{array}{l}1357 \\
(34 \%)\end{array}$ & $\begin{array}{l}2587 \\
(66 \%)\end{array}$ & $\begin{array}{l}2330 \\
(31 \%)\end{array}$ & $\begin{array}{l}5138 \\
(69 \%)\end{array}$ \\
\hline $\begin{array}{c}1991- \\
2015\end{array}$ & $\begin{array}{l}4272 \\
(29 \%)\end{array}$ & $\begin{array}{c}10701 \\
(71 \%)\end{array}$ & $\begin{array}{l}5005 \\
(29 \%)\end{array}$ & $\begin{array}{c}12067 \\
(71 \%)\end{array}$ & $\begin{array}{l}9395 \\
(29 \%)\end{array}$ & $\begin{array}{c}23190 \\
(71 \%)\end{array}$ \\
\hline
\end{tabular}

For the last 25 years the percentage of US citizen women still seeking employment has been a bit lower than that of men, though the opposite holds among non-US citizens, as shown in Table 2 . The unemployment

\footnotetext{
$\overline{{ }^{1} \text { When social scientists and statisticians study the effects of two }}$ independent variables and their interaction, they use the mathematical symbol $\times$ to denote testing for an interaction effect.

${ }^{2}$ Our full study can be found online at arXiv:1710:11256v1 [math. HO] In this summary, percentages are rounded for readability.
} 
rates were declining but increased for all categories in 2012-2015. We focused on the new PhDs who remained in the US after receiving their degrees and were still seeking employment at the time of the survey. (During 1991-2000 citizenship was unknown for some in this group, but they appear in the All PhDs column.) Following current AMS conventions on unemployment rate calculations, individuals employed outside the US as well as those whose employment status was unknown have been removed from the denominator in the calculation of the unemployment rate. We also adopt the AMS convention of removing those individuals reported as not seeking employment from the denominator. For the entire 25 years under investigation, this group accounted for $1 \%$ of all new PhDs (2\% of the females and $1 \%$ of the males). As pointed out in the 2015 survey, these conventions increase the unemployment rate from the rates reported prior to these adjustments.

Table 2. Number of New PhDs Still Seeking Employment by Citizenship and Gender (Percentages of all New PhDs in Column Cohort, denominators adjusted downward as described.)

Looking at gender $\times$ citizenship differences, Table 2

\begin{tabular}{|c|c|c|c|c|c|c|}
\hline & \multicolumn{2}{|c|}{ US } & \multicolumn{2}{c|}{ Non-US } & \multicolumn{2}{c|}{ All PhDs } \\
\hline Period & F & M & F & M & F & M \\
\hline $\begin{array}{c}1991- \\
2000\end{array}$ & $61(5 \%)$ & $271(9 \%)$ & $84(11 \%)$ & $304(11 \%)$ & $154(8 \%)$ & $595(10 \%)$ \\
\hline $\begin{array}{c}2001- \\
2011\end{array}$ & $50(3 \%)$ & $196(5 \%)$ & $89(5 \%)$ & $151(4 \%)$ & $139(4 \%)$ & $347(5 \%)$ \\
\hline $\begin{array}{c}2012- \\
2015\end{array}$ & $41(5 \%)$ & $176(8 \%)$ & $61(6 \%)$ & $85(5 \%)$ & $102(6 \%)$ & $261(7 \%)$ \\
\hline $\begin{array}{c}1991- \\
2015\end{array}$ & $152(4 \%)$ & $643(7 \%)$ & $234(7 \%)$ & $540(7 \%)$ & $395(5 \%)$ & $1203(7 \%)$ \\
\hline
\end{tabular}

shows that the disadvantage for non-US citizens from 1991-2000 is more pronounced for women; the rate for female non-US citizens was more than double the rate for citizens. The disadvantage for non-US citizen women lessened during 2001-2015. Notice that for male PhDs during 2001-2015, the unemployment rate for citizens was higher than for non-US citizens, with the greatest difference occurring in 2012-2015.

Women are less likely to have initial employment at top universities and in business and industry than men, as shown in Tables 3 and 4. In this summary we focused on pure math PhDs. The All Others rows in Tables 3 and 4 include new PhDs who accepted jobs in statistics, biostatistics, or applied math departments, outside the US, those who were still seeking or not seeking employment, as well as those whose employment status was unknown at the time of the survey. The AMS changed the annual survey reporting groupings in 2012 so we present data separately for 2012-2015. Beginning in 2012 the top-ranking Group I departments were replaced by the top-producing Public Large and Private Large departments.
Table 3. Observed Frequencies of First Jobs (Percentages of Column Cohort) for Pure Math PhDs 1991-2011

\begin{tabular}{|c|c|c|c|c|c|c|c|c|c|}
\hline & \multicolumn{3}{|c|}{$1991-2000$} & \multicolumn{3}{|c|}{$2001-2011$} & \multicolumn{3}{|c|}{ 1991-2011 } \\
\hline $\begin{array}{l}\text { Emp } \\
\text { Type }\end{array}$ & $\mathbf{F}$ & $\mathbf{M}$ & All & $\mathbf{F}$ & $\mathbf{M}$ & All & $\mathbf{F}$ & $\mathbf{M}$ & All \\
\hline Gr I & $\begin{array}{c}199 \\
(11 \%)\end{array}$ & $\begin{array}{c}849 \\
(13 \%)\end{array}$ & $\begin{array}{l}1048 \\
(13 \%)\end{array}$ & $\begin{array}{c}335 \\
(13 \%)\end{array}$ & $\begin{array}{l}1216 \\
(17 \%)\end{array}$ & $\begin{array}{l}1551 \\
(16 \%)\end{array}$ & $\begin{array}{c}534 \\
(12 \%)\end{array}$ & $\begin{array}{l}2065 \\
(15 \%)\end{array}$ & $\begin{array}{l}2599 \\
(15 \%)\end{array}$ \\
\hline Gr II & $\begin{array}{c}88 \\
(5 \%)\end{array}$ & $\begin{array}{l}326 \\
(5 \%)\end{array}$ & $\begin{array}{l}414 \\
(5 \%)\end{array}$ & $\begin{array}{l}193 \\
(8 \%)\end{array}$ & $\begin{array}{l}559 \\
(8 \%)\end{array}$ & $\begin{array}{l}752 \\
(8 \%)\end{array}$ & $\begin{array}{l}281 \\
(7 \%)\end{array}$ & $\begin{array}{l}885 \\
(7 \%)\end{array}$ & $\begin{array}{c}1166 \\
(7 \%)\end{array}$ \\
\hline Gr III & $\begin{array}{c}99 \\
(6 \%)\end{array}$ & $\begin{array}{l}251 \\
(4 \%)\end{array}$ & $\begin{array}{l}350 \\
(4 \%)\end{array}$ & $\begin{array}{l}120 \\
(5 \%)\end{array}$ & $\begin{array}{l}263 \\
(4 \%)\end{array}$ & $\begin{array}{l}383 \\
(4 \%)\end{array}$ & $\begin{array}{l}219 \\
(5 \%)\end{array}$ & $\begin{array}{l}514 \\
(4 \%)\end{array}$ & $\begin{array}{l}733 \\
(4 \%)\end{array}$ \\
\hline Masters & $\begin{array}{l}161 \\
(9 \%)\end{array}$ & $\begin{array}{l}416 \\
(7 \%)\end{array}$ & $\begin{array}{l}577 \\
(7 \%)\end{array}$ & $\begin{array}{l}202 \\
(8 \%)\end{array}$ & $\begin{array}{l}351 \\
(5 \%)\end{array}$ & $\begin{array}{l}553 \\
(6 \%)\end{array}$ & $\begin{array}{l}363 \\
(8 \%)\end{array}$ & $\begin{array}{l}767 \\
(6 \%)\end{array}$ & $\begin{array}{c}1130 \\
(6 \%)\end{array}$ \\
\hline $\begin{array}{c}\text { Bache- } \\
\text { lors }\end{array}$ & $\begin{array}{c}370 \\
(21 \%)\end{array}$ & $\begin{array}{c}787 \\
(12 \%)\end{array}$ & $\begin{array}{l}1157 \\
(14 \%)\end{array}$ & $\begin{array}{c}483 \\
(19 \%)\end{array}$ & $\begin{array}{c}877 \\
(12 \%)\end{array}$ & $\begin{array}{l}1360 \\
(14 \%)\end{array}$ & $\begin{array}{c}853 \\
(20 \%)\end{array}$ & $\begin{array}{l}1664 \\
(12 \%)\end{array}$ & $\begin{array}{l}2517 \\
(14 \%)\end{array}$ \\
\hline $2 \mathrm{Yr}$ & $\begin{array}{c}38 \\
(2 \%)\end{array}$ & $\begin{array}{l}110 \\
(2 \%)\end{array}$ & $\begin{array}{l}148 \\
(2 \%)\end{array}$ & $\begin{array}{c}61 \\
(2 \%)\end{array}$ & $\begin{array}{l}150 \\
(2 \%)\end{array}$ & $\begin{array}{l}211 \\
(2 \%)\end{array}$ & $\begin{array}{c}99 \\
(2 \%)\end{array}$ & $\begin{array}{l}260 \\
(2 \%)\end{array}$ & $\begin{array}{l}359 \\
(2 \%)\end{array}$ \\
\hline $\begin{array}{c}\text { Oth } \\
\text { Acad }\end{array}$ & $\begin{array}{c}61 \\
(3 \%)\end{array}$ & $\begin{array}{l}239 \\
(4 \%)\end{array}$ & $\begin{array}{l}300 \\
(4 \%)\end{array}$ & $\begin{array}{l}190 \\
(7 \%)\end{array}$ & $\begin{array}{l}407 \\
(6 \%)\end{array}$ & $\begin{array}{l}597 \\
(6 \%)\end{array}$ & $\begin{array}{l}251 \\
(6 \%)\end{array}$ & $\begin{array}{l}646 \\
(5 \%)\end{array}$ & $\begin{array}{l}897 \\
(5 \%)\end{array}$ \\
\hline $\begin{array}{l}\text { Res } \\
\text { Inst }\end{array}$ & $\begin{array}{c}32 \\
(2 \%)\end{array}$ & $\begin{array}{l}176 \\
(3 \%)\end{array}$ & $\begin{array}{l}208 \\
(3 \%)\end{array}$ & $\begin{array}{c}70 \\
(3 \%)\end{array}$ & $\begin{array}{l}134 \\
(2 \%)\end{array}$ & $\begin{array}{l}204 \\
(2 \%)\end{array}$ & $\begin{array}{l}102 \\
(2 \%)\end{array}$ & $\begin{array}{l}310 \\
(2 \%)\end{array}$ & $\begin{array}{l}412 \\
(2 \%)\end{array}$ \\
\hline Govt & $\begin{array}{c}36 \\
(2 \%)\end{array}$ & $\begin{array}{l}141 \\
(2 \%)\end{array}$ & $\begin{array}{l}177 \\
(2 \%)\end{array}$ & $\begin{array}{c}90 \\
(4 \%)\end{array}$ & $\begin{array}{l}202 \\
(3 \%)\end{array}$ & $\begin{array}{l}292 \\
(3 \%)\end{array}$ & $\begin{array}{l}126 \\
(3 \%)\end{array}$ & $\begin{array}{l}343 \\
(3 \%)\end{array}$ & $\begin{array}{l}469 \\
(3 \%)\end{array}$ \\
\hline $\begin{array}{l}\text { Bus/ } \\
\text { Ind }\end{array}$ & $\begin{array}{l}143 \\
(8 \%)\end{array}$ & $\begin{array}{c}754 \\
(12 \%)\end{array}$ & $\begin{array}{c}897 \\
(11 \%)\end{array}$ & $\begin{array}{l}205 \\
(8 \%)\end{array}$ & $\begin{array}{c}692 \\
(10 \%)\end{array}$ & $\begin{array}{l}897 \\
(9 \%)\end{array}$ & $\begin{array}{l}348 \\
(8 \%)\end{array}$ & $\begin{array}{l}1446 \\
(11 \%)\end{array}$ & $\begin{array}{l}1794 \\
(10 \%)\end{array}$ \\
\hline $\begin{array}{c}\text { All } \\
\text { Others }\end{array}$ & $\begin{array}{c}555 \\
(31 \%)\end{array}$ & $\begin{array}{l}2324 \\
(36 \%)\end{array}$ & $\begin{array}{l}2879 \\
(35 \%)\end{array}$ & $\begin{array}{c}602 \\
(24 \%)\end{array}$ & $\begin{array}{l}2196 \\
(31 \%)\end{array}$ & $\begin{array}{l}2798 \\
(29 \%)\end{array}$ & $\begin{array}{l}1157 \\
(27 \%)\end{array}$ & $\begin{array}{l}4520 \\
(34 \%)\end{array}$ & $\begin{array}{l}5677 \\
(32 \%)\end{array}$ \\
\hline $\begin{array}{c}\text { Grand } \\
\text { Total }\end{array}$ & $\begin{array}{c}1782 \\
(100 \%)\end{array}$ & $\begin{array}{c}6373 \\
(100 \%)\end{array}$ & $\begin{array}{c}8155 \\
(100 \%)\end{array}$ & $\begin{array}{c}2551 \\
(100 \%)\end{array}$ & $\begin{array}{c}7047 \\
(100 \%)\end{array}$ & $\begin{array}{c}9598 \\
(100 \%)\end{array}$ & $\begin{array}{c}4333 \\
(100 \%)\end{array}$ & $\begin{array}{l}13,420 \\
(100 \%)\end{array}$ & $\begin{array}{l}17,753 \\
(100 \%)\end{array}$ \\
\hline
\end{tabular}

During all periods of our study there were striking gender differences between the percentages of male and female new pure math PhDs employed in departments in which the highest mathematics degree is a bachelor's. We first refer to Table 3 for our observations. Between 1991 and 2000 the percentage of women who were employed at bachelor's-only departments was $21 \%$ vs. $12 \%$ for men; between 2001 and 2011 slightly less but still large; during 2012-2015 (Table 4), 18\% vs. 10\%, the largest relative difference we observed.

During 1991-2000 (Table 3), the percentage of women who were employed at top-ranking Group I departments was $11 \%$ vs. $13 \%$ for men; during $2001-2011,13 \%$ vs. $17 \%$ for men. To put this in perspective, during 1991-2011, $47 \%$ of all women pure math $\mathrm{PhDs}$ received degrees from Group I institutions and 56\% of the men pure math $\mathrm{PhDs}$ received degrees from Group I institutions. Looking only at Group I PhDs during 1991-2011, 24\% were women.

Similarly during 2012-2015 (Table 4) the percentage of women who were employed at Public and Private Large departments was considerably lower than for men, with the biggest difference occurring in Private Large hires. 
Table 4. Observed Frequencies of First Jobs (Percentages of Column Cohort) for Pure Math PhDs 2012-2015

\begin{tabular}{|c|c|c|c|}
\hline Employer Type & Female & Male & All \\
\hline Public Large & $\begin{array}{c}94 \\
(7 \%) \\
\end{array}$ & $\begin{array}{l}332 \\
(9 \%) \\
\end{array}$ & $\begin{array}{l}426 \\
(9 \%) \\
\end{array}$ \\
\hline Public Medium & $\begin{array}{c}60 \\
(5 \%) \\
\end{array}$ & $\begin{array}{l}179 \\
(5 \%) \\
\end{array}$ & $\begin{array}{l}239 \\
(5 \%) \\
\end{array}$ \\
\hline Public Small & $\begin{array}{c}51 \\
(4 \%) \\
\end{array}$ & $\begin{array}{l}129 \\
(4 \%) \\
\end{array}$ & $\begin{array}{l}180 \\
(4 \%)\end{array}$ \\
\hline Private Large & $\begin{array}{c}55 \\
(4 \%)\end{array}$ & $\begin{array}{l}230 \\
(7 \%)\end{array}$ & $\begin{array}{l}285 \\
(6 \%) \\
\end{array}$ \\
\hline Private Small & $\begin{array}{c}29 \\
(2 \%) \\
\end{array}$ & $\begin{array}{c}62 \\
(2 \%)\end{array}$ & $\begin{array}{c}91 \\
(2 \%)\end{array}$ \\
\hline Masters & $\begin{array}{c}53 \\
(4 \%) \\
\end{array}$ & $\begin{array}{l}121 \\
(3 \%) \\
\end{array}$ & $\begin{array}{l}174 \\
(4 \%)\end{array}$ \\
\hline Bachelors & $\begin{array}{c}222 \\
(18 \%) \\
\end{array}$ & $\begin{array}{c}354 \\
(10 \%) \\
\end{array}$ & $\begin{array}{c}576 \\
(12 \%) \\
\end{array}$ \\
\hline 2 Year & $\begin{array}{c}35 \\
(3 \%) \\
\end{array}$ & $\begin{array}{c}80 \\
(2 \%) \\
\end{array}$ & $\begin{array}{l}115 \\
(2 \%) \\
\end{array}$ \\
\hline Other Academic & $\begin{array}{c}91 \\
(7 \%) \\
\end{array}$ & $\begin{array}{l}197 \\
(6 \%) \\
\end{array}$ & $\begin{array}{l}288 \\
(6 \%) \\
\end{array}$ \\
\hline Research Inst & $\begin{array}{c}25 \\
(2 \%) \\
\end{array}$ & $\begin{array}{c}87 \\
(2 \%) \\
\end{array}$ & $\begin{array}{l}112 \\
(2 \%) \\
\end{array}$ \\
\hline Government & $\begin{array}{c}52 \\
(4 \%) \\
\end{array}$ & $\begin{array}{l}109 \\
(3 \%) \\
\end{array}$ & $\begin{array}{l}161 \\
(3 \%) \\
\end{array}$ \\
\hline Business/Industry & $\begin{array}{c}171 \\
(14 \%) \\
\end{array}$ & $\begin{array}{c}577 \\
(16 \%) \\
\end{array}$ & $\begin{array}{c}748 \\
(16 \%) \\
\end{array}$ \\
\hline All Others & $\begin{array}{c}328 \\
(26 \%) \\
\end{array}$ & $\begin{array}{r}1095 \\
(31 \%) \\
\end{array}$ & $\begin{array}{l}1423 \\
(30 \%) \\
\end{array}$ \\
\hline Grand Total & $\begin{array}{c}1266 \\
(100 \%)\end{array}$ & $\begin{array}{c}3552 \\
(100 \%)\end{array}$ & $\begin{array}{c}4818 \\
(100 \%)\end{array}$ \\
\hline
\end{tabular}

Our full study looked at the types of employment obtained by the various groups of new PhDs, not just pure mathematics PhDs.

Women are slightly less likely to be employed by departments whose ranking is at least comparable to the degree-granting department. We considered jobs at Research Institutes or Other Non-Profits as desirable and group them with the top-ranking or top-producing departments (refer to Table 5). As in past studies [3], we note that since the data collected from departments does not give detailed information on the type of position, a definitive statement is not possible. Table 5 shows that the at least comparable employment rates ${ }^{3}$ for both females and males from Group II institutions improved between 2001 and 2011.

\footnotetext{
${ }^{3}$ For each rate, we calculated the percent who obtained jobs at departments ranked at least as high as the degree-granting department or at Research Institutes/Other Non-Profits.
}

Table 5. At Least Comparable Employment Rates for New Pure Math PhDs

\begin{tabular}{|c|c|c|c|c|c|c|c|c|c|c|c|c|}
\hline \multicolumn{7}{|c|}{ PhD Granting Institution } \\
\hline \multicolumn{7}{|c|}{$1991-2011$} & \multicolumn{7}{c|}{$2001-2011$} \\
\hline Group I & \multicolumn{2}{|c|}{ Group II } & Group III & \multicolumn{2}{|c|}{ Group I } & \multicolumn{2}{|c|}{ Group II } & \multicolumn{2}{c|}{ Group III } \\
\hline F & M & F & M & F & M & F & M & F & M & F & M \\
\hline 22\% & $25 \%$ & $13 \%$ & $13 \%$ & $14 \%$ & $12 \%$ & $29 \%$ & $29 \%$ & $18 \%$ & $19 \%$ & $15 \%$ & $18 \%$ \\
\hline \multicolumn{7}{|c|}{$25 \%$} & 2012-2015 \\
\hline Public Large & Private Large & Public Medium & Public Small & Private Small \\
\hline F & M & F & M & F & M & F & M & F & M \\
\hline 22\% & $25 \%$ & $31 \%$ & $32 \%$ & $15 \%$ & $19 \%$ & $16 \%$ & $19 \%$ & $28 \%$ & $25 \%$ \\
\hline
\end{tabular}

During 1991-2000, the at least comparable employment rate for women from the top Group I programs was 3 points less than men (about 12\% less than the men's rate) while the rate for women from Group III programs was 2 points higher than the men's rate (roughly $17 \%$ higher than the men's rate). During 2001-2011, the gender difference in Group III degree recipients reversed itself and other gender differences were reduced. Between 2012 and 2015 women from Public Large programs were 12\% less likely than men to be employed by an at least comparable department, while women from Public and Private Small were about $16 \%$ and $12 \%$, respectively, more likely than men to be hired by an at least comparable department.

\section{Notes on the Data}

Each year the American Mathematical Society (AMS) conducts a census of new PhDs by sending surveys to all departments that grant doctoral degrees in mathematics, statistics, biostatistics, and applied mathematics, as well as follow-ups to all $\mathrm{PhD}$ recipients. Over the years there have been changes in what data is collected and how it is reported. Between 1991 and 2011 the AMS reported data for doctorate-granting pure mathematics departments partitioned into Groups I, II, and III, based on the then current ranking of US doctoral departments as determined by the National Research Council (NRC), a part of the National Academies of Science. Starting in 1996, Group I was subdivided into Group I Public and Group I Private and Groups IV and V were added. Group IV consisted of statistics and biostatistics programs and Group V applied mathematics and operations research programs. We exclude doctorates in operations research (Group Vb) from our current study since the AMS has not collected data on these degrees for several years.

The NRC released reports and rankings of research doctoral programs in 1982, 1995, and 2010. Prior to 2012, the AMS followed the recommendations of the Joint Data Committee to use these rankings to create three groups of 
pure mathematics doctoral programs, ${ }^{4}$ with Group I comprised of the top-rated programs. The 2010 NRC report [2] does not give a single ranking of programs but rather ranks programs on five different scales with each score presented as a range of rankings; the scales are based on 20 characteristics. Starting in 2012, upon the advice of the Joint Data Committee, the AMS partitioned the pure mathematics departments into Math Public Large, Math Public Medium, Math Public Small, Math Private Large, and Math Private Small. This subdivision was based solely on the number of $\mathrm{PhDs}$ produced by the departments as reported on the annual surveys. Lists of the departments in these groups as well as a comparison with the former groups can be found on the AMS website [1]. Due to this change, we did separate analyses for the time periods 1991-2011 and 2012-2015.

The response rate for all groups treated in this report has been very high; the 2015 Annual Survey reports that information was provided by 312 of the 318 doctoral-granting departments queried. Survey response rates by grouping are reported by the AMS in the annual surveys published in the Notices of the American Mathematical Society and available online. Despite the high overall response rate, over the past several years an increasing number of departments have sent the AMS only basic information on their new PhDs and have often omitted data on employment status. The number of unknowns would be even higher but for web searches by the AMS that secured additional employment information, especially for those in academia. This is among the reasons why the AMS conjectures new PhDs who are categorized as Unknowns are skewed toward new PhDs in non-academic employment and individuals who may no longer be in the US. The survey data also do not either distinguish between one-year and multi-year jobs or identify tenure-stream positions.

\section{References}

1. Annual Survey of the Mathematical Sciences.www.ams.org/ profession/data/annual-survey/survey-reports.

2. National Research Council. A Data-Based Assessment of Research-Doctorate Programs in the United States, 2011. The National Academies Press: Washington, DC. doi:10.17226/12994. www.nap.edu/rdp/

3. M. A. Vitulli and M. E. Flahive 1997. Are Women Getting All The Jobs? Notices Amer. Math Soc. (March 1997), www. ams .org/ notices/199703/comm-vitu11i.pdf; An Update, Notices Amer. Math. Soc. (Sept. 2010), www. ams.org/profession/ data/other-sources/Are-Women-Getting-A17-Jobs.pdf.
ACKNOWLEDGMENT. We wish to thank Thomas H. Barr and Colleen A. Rose of the American Mathematical Society for supplying the data collected from the surveys and for answering questions about the data.

\section{ABOUT THE AUTHOR}

Marie A. Vitulli's main research interests are in commutative algebra and algebraic geometry, particularly in the theories of seminormality, weak normality, and valuations on commutative rings. In 2014 she delivered the AWM-MAA Etta Z. Falconer Lecture at MAA MathFest. She created and maintains the award-winning website Women in Math Project pages . uoregon . edu/ wmnmath.

\footnotetext{
$\overline{{ }^{4} \text { When we speak of pure mathematics departments we exclude }}$ departments in applied mathematics, statistics, and biostatistics.
} 


\title{
Writing Women in Mathematics into Wikipedia
}

\author{
Marie A. Vitulli
}

\author{
Communicated by Harriet Pollatsek
}

ABSTRACT. In this article I reflect upon the problems connected with writing women in mathematics into Wikipedia. I hope to persuade the reader to start editing existing Wikipedia articles and to create new articles. I also hope to persuade existing editors to contribute new articles about women mathematicians.

The twin problems of the paucity of women subjects and scarcity of women editors on Wikipedia are well known, but no solutions are on the horizon. We can nevertheless take small steps to address these problems. In response to my concerns about these problems and in order to gain first-hand experience, I became a Wikipedia editor in 2013. In the first section, I discuss gender bias on Wikipedia and the underrepresentation of women subjects. In the second section, I discuss the scarcity of women contributors and my personal experiences of the difficulties women contributors encounter. In the third section, I discuss some recent steps taken towards solving the problems, including the Wikipedia Year of Science, the related projects WikiProject Women scientists and WikiProject Women in Red, and a recent partnership between the Association for Women in Mathematics (AWM) and the Wiki Education Foundation to write and improve pages on women mathematicians. Advice for writing a biography on Wikipedia may be found in a longer, posted version of this article [3].

Marie A. Vitulli is professor emerita at the University of Oregon. Her email address is vi tu $11 \mathrm{i} @ u$ uregon. edu. This article updates and expands upon an article in the May-June 2014 AWM Newsletter www . drivehq. com/folder/p8755087/11874396230.aspx.

For permission to reprint this article, please contact: reprint-permission@ams . org.

DOI: http://dx.doi.org/10.1090/noti1650

\section{The Paucity of Women Subjects}

There have been numerous allegations that Wikipedia suffers from systemic gender bias with respect to both content and editors and that the attempts to increase women's participation have failed. ${ }^{1}$

One disturbing manifestation of the underrepresentation of women in Wikipedia is the dearth of biographies of notable women. According to WikiProject Women in Red, only $16.36 \%$ of the biographies in English Wikipedia were about women as of August 7, 2016. This is up from just over 15\% in November 2014. Wikipedia has guidelines on academic notability (also called the professor test) that a subject must meet to merit a Wikipedia page. The first two of the nine criteria of academic notability are: the person's research has made significant impact in their scholarly discipline, broadly construed, as demonstrated by independent reliable sources; and the person has received a highly prestigious academic award or honor at a national or international level. Some of the criteria are subjective in nature, and it is not surprising that some of the American mathematicians featured on Wikipedia are more notable than others.

In 2013 the National Science Foundation awarded a grant ${ }^{2}$ to Yale University to explore potential gender differences in indicators of academic notability, networks that facilitate content creation, gate-keeping processes that challenge content associated with women and scholarship, and the unintended consequences of Wikipedia's policies.

When I first perused the page for the "Category: Women mathematicians" (formerly called "Category: Female mathematicians") several years ago, I was disheartened to see how many prominent women lacked a Wikipedia page.

${ }^{1}$ For references and further discussion, see the Wikipedia page "Gender bias on Wikipedia" en.wikipedia.org/wiki/ Gender_bias_on_Wikipedia.

${ }^{2}$ NSF Sociology, Science, Technology \& Society Program Award www.nsf.gov/awardsearch/showAward?AWD_ID=1322934. 
Comparing the category lists in August of 2015 and August of 2016, I was pleased to discover that there were 73 new biographies of women mathematicians on Wikipedia.

I also keep an eye on the women included in the "Category: American mathematicians." As of August 30, 2016, there were thirty-seven pages (people) in this category. If we assume every woman in the "Category: American women mathematicians" is also in the "Category: American mathematicians" (which is not the case ${ }^{3}$ ), the percentage of women in the "Category: American mathematicians" was about $17 \%$ at that time. For comparison, according to the AMS Annual Survey, we know that 29\% of the US citizens to earn PhDs from US mathematics departments between 1991 and 2015 were women. As per the 2015 AMS Annual Survey, about 23\% of all full-time doctoral mathematics faculty were women, and 19\% of the Public or Private Large (the top-producing departments) doctoral mathematics faculty were women. I am happy to report that the number of women listed in the "Category: American women mathematicians" nearly doubled between August 2016 and October 2017 (from 37 to 73), in part due to the efforts I'll discuss in the next section.

One deterrent to the creation of more biographies of women mathematicians on Wikipedia is the scarcity of women editors. Another serious impediment is that not all of the articles that are created will "survive" on Wikipedia. Both of these hindrances will be discussed briefly in Section 2. Please see my longer article [3] for more details.

\section{The Scarcity of Women Wikipedia Editors and My Personal Experiences}

Women are substantially underrepresented on Wikipedia as editors (see [2]). A survey conducted by the United Nations University and Maastricht University in collaboration with the Wikimedia Foundation demonstrated that in 2008 only $12.64 \%$ of Wikipedia editors worldwide were women. A follow-up survey in 2011 showed that things hadn't improved: $9 \%$ of editors worldwide and $15 \%$ of those from the US were women. A few years after the initial study, the Wikimedia Foundation announced the goal of raising the proportion of female editors worldwide to 25\% by 2015. In 2014 Wikimedia Foundation founder Jimmy Wales announced that Wikipedia had completely failed to reach this goal despite launching several initiatives. This is particularly disturbing in light of the fact that the percentage of adult Americans who use Wikipedia for information increased from 25\% in February 2007 to $42 \%$ in May 2010, according to a study [1] conducted by the Pew Research Center. The Pew study also showed that about $50 \%$ of the adult female Internet users and $56 \%$ of the male adult Internet users look to Wikipedia for information.

In order to better understand the potential problems an editor faces, I attended my first edit-a-thon on writing women into Wikipedia at the University of Oregon in March of 2013. This event was part of the Wiki Women's History Month events and was led by Sarah Stierch, who

\footnotetext{
${ }^{3}$ It is up to an editor to decide what categories a page belongs to. Sometimes categories that have been assigned by one editor are removed by another editor.
}

was then a program evaluation coordinator for the Wikimedia Foundation and a former Wikipedian in Residence at the Archives of American Art and the Smithsonian Institution Archives.

During the edit-a-thon I decided to write an article about Susan Montgomery. Montgomery was the 2011 AWM Noether Lecturer, and in 2012 she was selected as both an AAAS Fellow and an AMS Fellow in the inaugural class. I felt she clearly merited a page on Wikipedia. I began working on her page during the edit-a-thon and finished up the article a couple of weeks later. My first experience writing for Wikipedia was extremely frustrating, but in the end I was glad that I undertook this project. I learned that there are many Wikipedia conventions that a contributor must follow. I am still learning about these conventions and the culture of Wikipedia.

The first thing I did during the edit-a-thon was to edit the existing Wikipedia page on the Noether Lecture so that the lecturers for 2011-2013, including Montgomery, were listed on that page. I inserted square brackets around Montgomery's name so she appeared as a red link, that is, a person (or topic) mentioned on Wikipedia for whom a Wikipedia page does not exist. ${ }^{4}$ A biography has a better chance of surviving on Wikipedia if the subject is already mentioned on an existing Wikipedia page. I followed all the above-mentioned guidelines and asked Stierch to read my article before I published it. In spite of all my precautions, my article was proposed for deletion with the PROD (Proposed deletion) tag within ten minutes after it went live by a novice editor who made this remark:

It is proposed that this article be deleted because of the following concern: This looks like a case of shameless (self) promotion. Hey, look at me: I teach math and I want my CV on Wikipedia.

Stierch responded to the novice editor by saying the biography was in fact a good faith article by a new editor (not the subject) and removed the PROD tag. The article survived only because of Stierch's intervention. ${ }^{5}$

At Montgomery's request, I started another Wikipedia biography in May of 2014, this one on Georgia Benkart.

\footnotetext{
${ }^{4}$ A Wikipedia editor can easily create a red link on Wikipedia by surrounding [[name]] or [[topic]] with double square brackets as indicated.

${ }^{5}$ Stierch, whose username today is Missvain, is still an active Wikipedia administrator. Regrettably, Stierch lost her Wikimedia Foundation job in late 2013 when she was discovered editing for pay, see arstechnica.com/tech-policy/2014/01/wikimediafoundation-employee-ousted-over-paid-editing, In general there is a prohibition on paid editing, but it isn't uniformly enforced. I have heard that the Ivy League schools have public relations staff who create pages for distinguished faculty; the staff are supposed to acknowledge their employer in their "Talk" pages. I came across one such acknowledgment on the "User" page of Dominic McDevitt-Parks (User: Dominic), who is a paid employee of the US National Archives and Records Administration and edits on Wikipedia for them. I suspect that there are many other paid editors who make no such acknowledgment.
} 
Benkart was AWM President 2009-2011, was elected to the inaugural class of Fellows of the AMS in 2013, and was selected to deliver the AWM-AMS Noether Lecture in 2014 and the Emmy Noether Lecture at the International Congress of Mathematicians in Seoul, Korea, in 2014. She has published over 100 journal articles and has co-authored three Memoirs of the American Mathematical Society. Benkart clearly met the Wikipedia criteria for academic notability. I had Stierch both read my article on Benkart and later monitor it when the article was published on Wikipedia. I also asked Emily Temple-Wood to watch the page and make sure it wasn't deleted.

Soon after the Benkart biography went live, it was tagged for having too many primary sources and not enough secondary sources. Wikipedia recommends that a biography should have at least as many secondary sources in the "References" section as publications of the subject, which are regarded as primary sources. I addressed that criticism and removed the tag after thanking the editor involved for his comments and explaining that most of the sources were now secondary. The article was then tagged for using weasel words: vague phrasing that often accompanies biased or unverifiable information. The Wikipedia administrator asserted the following:

The whole tone of the article is written putting her on a pedestal.

After asking Emily Temple-Wood and Sarah Stierch about weasel words and looking at the "Talk" page for the article, I found out that the administrator was objecting to my claims that Benkart was a "distinguished" mathematician who is an "international leader" and a "renowned" teacher, and for asserting that one of Benkart's joint papers became "one of the building blocks" of the classification of toroidal rank-one Lie algebras. Since I am not an expert in Benkart's research area (the structure and representation theory of Lie algebras), I asked Montgomery for help. Montgomery enlisted the help of Efim Zelmanov, who wrote up the paragraphs that described the "importance" of Benkart's work (I couldn't help slipping in the weasel word "importance"). In this case an administrator who has virtually no background in mathematics objected to a Fields Medalist's description of Benkart's work. As Stierch pointed out to me, this is the reality of the wacky world of Wikipedia. If an editor can't prove her assertion with a reliable secondary source, then any other editor can remove the relevant sentence without question. Stierch removed the uncited weasel words from my article and the tag was eventually removed. As of this writing, the article has survived on Wikipedia.

Other mathematicians have had a difficult time when writing biographies of women mathematicians. For example, Christina Sormani has created several biographies, originally with the username "Sormani." Beginning around 2007 , the articles she wrote were deleted by other editors and Administrators. She then started writing articles anonymously without logging on to the Wikipedia site and had more success. We do not have any data, numerical or anecdotal, on the difficulties editors have in creating or editing pages on male mathematicians.

\section{Steps Toward Improving the Situation}

During the past several years there have been a number of successful attempts to increase the visibility of women mathematicians on Wikipedia. In 2007 the Wikipedia page "Noether Lecture" was created by AWM member Sormani. The Noether Lecture is a lecture series (and award) that honors women "who have made fundamental and sustained contributions to the mathematical sciences." The AWM established the lecture in 1980 and in 2013 it was renamed the AWM-AMS Noether Lecture. The Noether lecturers were all listed on the page Noether Lecture, some as red links (without their own Wikipedia pages). I am happy to report that as of today all of the Noether lecturers listed on the page "Noether Lecture" appear as blue links (with pages on Wikipedia).

The WikiProject Women scientists (Figure 1) began in 2012 and is dedicated to ensuring the quality and quantity of biographies of women in science on Wikipedia. Emily Temple-Wood (who edits on Wikipedia as Keilana) is a co-founder of this project; she started writing Wikipedia pages when she was twelve and continues to write pages and confront gender bias on Wikipedia, although her pace has slowed a bit since she began medical school at Midwestern University in the fall of 2016. Temple-Wood has written over 100 pages on women scientists. She became an administrator in 2007 and is currently part of the Arbitration Committee. There is a plethora of information on the project page for WikiProject Women scientists, including suggestions about how an editor can help the project. There are links to pages that are helpful to Wikipedia editors and a list of more than 80 members of the project. The list of members begins with the usernames of Emily Temple-Wood and Sarah Stierch (Keilana and SarahStierch, respectively); I am member 84 near the bottom of the list.

The objective of the WikiProject Women in Red is to turn red links of women on Wikipedia into blue links.

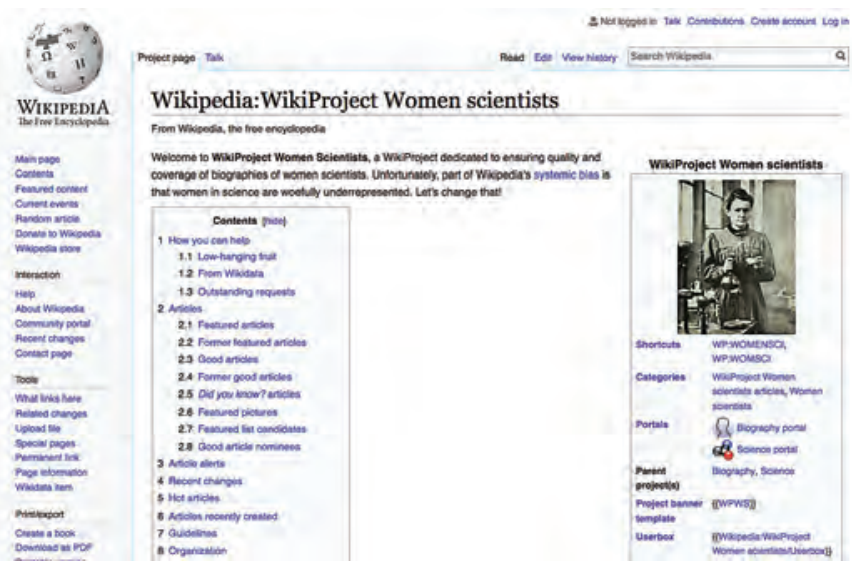

Figure 1. WikiProject Women scientists includes suggestions about how an editor can help. 
Rosie Stephenson-Goodknight (who edits on Wikipedia as Rosiestep) is a co-founder of this WikiProject. WikiProject Women in Red hosts edit-a-thons and publicizes its scope and objective via social media. WikiProject Women in Red has a page on "Mathematics," which is organized by country and lists missing articles on women who are (or have been) notable for their contributions to mathematics in academics, business, economics, politics, research, government, or the social sector. I made many additions to the list and encourage you to do so as well. To locate the pages of these WikiProjects one can use the special search box at en.wikipedia.org/wiki/Wikipedia:WikiProject.

Temple-Wood and Stephenson-Goodknight were named as 2016 co-Wikipedians of the Year by Wikipedia Trustee Jimmy Wales in recognition of their work on harassment on Wikipedia along with their collective efforts to expand coverage of notable women on Wikipedia.

The year 2016 was the Wikipedia Year of Science, an unparalleled initiative to improve scientific literacy through Wikipedia. A fundamental part of the Year of Science was the Women in Science initiative, the stated goal of which was to create or improve coverage of women scientists on Wikipedia. Three 2016 was the Wikipedia Year of Science. groups, WikiProject Women scientists, WikiProject Women in Red, and the Wiki Education Foundation, joined forces to run virtual edit-a-thons during the year to create and improve articles about women scientists in diverse fields, emphasizing a different area each month between February and December. From the parent page "Wikipedia: Year of Science," you can find a list of the featured areas and their home pages, which were designed to encourage Wikipedians to collaborate, share successes, identify articles that need to be written, find subject-specific sources, or initiate any activity that improves coverage of or celebrates women in science. The month of October highlighted computer science, technology, and mathematics, and the corresponding Wikipedia page included a section entitled "Biographies of Mathematicians To Create or Improve," which I authored. In this section, one can find a list of prominent women in mathematics who either don't have Wikipedia pages or whose pages are stubs (pages that need to be expanded to meet Wikipedia standards). The page also contains a section labeled "Association for Women in Mathematics" that lists AWM awards, prizes, and lectures that either don't have pages or some of whose recipients don't have pages. This page is a good starting point for someone who wants to edit or create a page enhancing the visibility of women in mathematics on Wikipedia.

In the recent past, there have been efforts to create pages for women elected as Fellows of the AMS or fellows of other major professional organizations. Formerly, on the "Talk" page of the "WikiProject: Women scientists," in a section entitled "Some missing mathematicians," there was a list of women who had been elected Fellows of the AMS but didn't have pages; soon after the list appeared, pages were created for all of these women, primarily by User:Brirush and User:David Eppstein. This wasn't done for the class of 2018 AMS Fellows. I created a page for Rachel Justine Pries. Almost immediately, another Wikipedia editor, User:FormalDude, attached the PROD tag to the article and proposed it for deletion. I successfully defended the notability of Pries and had a team of editors watch the page; to date the page has survived. Pages for 2018 Fellows Antonella Grassi and Hema Srinivasan were created by Ursula Whitcher and David Eppstein, respectively.

In the summer of 2016 Jami Mathewson of the Wiki Education Foundation created the page "Category: Awards and prizes of the Association for Women in Mathematics." As of October 12, 2017 there were four pages in this category: "AWM/MAA Falconer Lecturer," "Louise Hay Award," "M. Gweneth Humphreys Award," and "Noether Lecture." Some of the mathematicians listed on the first three pages appear as red links. The Wikipedia page Association for Women in Mathematics" lists all past and current AWM presidents. Despite the fact that all AWM presidents have Wikipedia pages, and hence appear as blue links, Mathewson reports that only one page (the page for Ruth Charney) has been rated as "B-class" by the WikiProjects Biography and Women scientists, designating it as one of Wikipedia's higher-quality articles. ${ }^{6}$ Many of the pages for former AWM presidents are stubs. To be fair, many of the pages are not rated. My point in mentioning this assessment is that there are still a lot of opportunities for work for fledgling editors who may not yet want to create a page from scratch.

Several women who have created biographies of women in mathematics on Wikipedia recommend that the author of a new page enlist the aid of existing editors to affirm the new article by making minor edits soon after the article goes live. Edits can be made by less senior editors without logging in to their Wikipedia accounts; only the IP address of the computer they were using will appear on the "History" page of the article. It is even better if a page creator has an active administrator looking out for a newly created article.

While writing this article I learned of an unprecedented partnership between the AWM and the Wiki Education Foundation. There is considerable need for such a partnership, particularly since there don't seem to be many mathematicians involved in the WikiProject Women scientists and the WikiProject Women in Red. As I said earlier, I added my name as a member of the WikiProject Women scientists, but I didn't see any other usernames that I recognized. In the past, I

\footnotetext{
${ }^{6}$ To see these ratings, click on the tabs for the "Talk" pages of the articles.
} 
have sought out Stierch and Temple-Wood for advice and for help protecting the pages I wrote. Neither of these allies has much time to devote to Wikipedia today. In response to a query I posted on Temple-Wood's “Talk" page, Susan Barnum, a public services librarian who lives in El Paso, Texas, and has the username Megalibrarygirl, offered help with copyediting and references. It is crucial that we add women editors who can create pages for prominent women in mathematics. I believe that this effort will fail unless there is a network of Wikipedia editors and administrators who watch the pages that we create. Hopefully the partnership can help build such a network.

In another unprecedented move, AWM member and associate editor at Mathematical Reviews Ursula Whitcher organized a Wikipedia women in mathematics edit-a-thon at the 2017 AWM Research Symposium, which was held on the campus of the University of California, Los Angeles. The edit-a-thon was supported by a grant from the Wiki Education Foundation. Mathewson and I assisted Whitcher during the edit-a-thon. Edit-a-thons are wonderful resources for novice editors. Ten new pages on women mathematicians were created during the AWM edit-a-thon, including a page I created on 2017 AMS Fellow Mei-Chu Chang. Please view and help improve these pages by visiting the Wikipedia page for the edit-a-thon.

\section{Summary}

There are many more Wikipedia articles on women mathematicians today than when I first became a Wikipedia editor in early 2013. However, many of these articles are stubs, and not a single woman mathematician appears in the ten articles allotted to mathematicians in "Wikipedia: Vital_Articles." WikiProject Women scientists is attempting to have the page on Emmy Noether included in this prestigious list. Although all of the women who were elected AMS Fellows now have Wikipedia pages, several of the women who were elected SIAM Fellows or who have received awards from national organizations still do not have pages. The percentage of women editors on Wikipedia remains dismally low.

I have edited many biographies of women mathematicians and will continue to do so. Even though writing a new biography is at times frustrating and is always time consuming, I am glad that I created biographies of four prominent women mathematicians and plan to write more. Anyone who wants to become a Wikipedia editor can find a useful list of Wikipedia help pages on the bottom of the "Project" page for the WikiProject Women scientists. Placing (WPWS) at the top of the "Talk" page of an article will result in the article being automatically added to "Category: WikiProject women scientists" and the other members of the WikiProject will be able to keep an eye on the page. I hope you will decide to take the plunge. Our entire community will be looking forward to reading new articles on notable women in mathematics.
ACKNOWLEDGMENT. I would like to acknowledge and applaud the efforts of Emily Temple-Wood and Rosie Stephenson-Goodknight for their work on harassment on Wikipedia along with their collective efforts to expand coverage of notable women on Wikipedia.

\section{References}

1. KAthryn and LeE RAinie, Wikipedia past and present: A snapshot of Wikipedia users. Pew Research Center. January 13, 2011, www. pewinternet.org/2011/01/13/wikipediapast-and-present.

2. NICOLE TORRES, Why do so few women edit Wikipedia? Harvard Business Review, June 2, 2016, https://hbr.org/2016/06/ why-do-so-few-women-edit-wikipedia.

3. MARIE A. VITULLI, Writing women mathematicians into Wikipedia (long version), 2017, arXiv: 1710.11103v3[math. HO].

Image Credits

Figure 1 from en.wikipedia.org/wiki/Wikipedia: WikiProject_Women_scientists. Photo of Marie A. Vitulli courtesy of Marie A. Vitulli.

\section{ABOUT THE AUTHOR}

Marie A. Vitulli's main research interests are in commutative algebra and algebraic geometry, particularly in the theories of seminormality, weak normality, and valuations on commutative rings. She has been concerned about the underrepresentation of women in mathematics and the inadequate recognition of outstanding female mathematicians since the late 1970s when she began her long career as a university professor of mathematics. 


\section{LETTERS TO THE EDITOR}

\section{Noether's Two Theorems}

I enjoyed reading the article by Fadi Bardawil et al. on "Immigration, Freedom, and the History of the Institute for Advanced Study," especially the substantial paragraph on Emmy Noether, in the November 2017 issue of the Notices. On the other hand, I regret that the Editor's Note giving a list of related Notices articles failed to reference Robyn Arianrhod's review ${ }^{1}$ of my book, The Noether Theorems: Invariance and Conservation Laws in the Twentieth Century. My book is not a biography of Noether; yet it contains essential biographical information and also clarifies the slightly misleading affirmation in the Notices article, "Noether's theorem not only laid this controversy to rest..."As pointed out long ago by Peter Olver, Noether's 1918 article contained two theorems. It is not "Noether's [first] theorem" but her second theorem that explained and vastly generalized an assertion of Hilbert, and thus "laid to rest" the controversy concerning the conservation of energy in Einstein's general theory of relativity.

-Yvette Kosmann-Schwarzbach École Polytechnique, retired Yvette.Kosmann-Schwarzbach@math.cnrs.fr

(Received November 5, 2017)

\footnotetext{
${ }_{1}^{1}$ Notices, August 2013, www. ams.org/notices/201307/rnotip916.pdf

${ }^{2}$ Bull. Amer. Math. Soc., 2013, www.ams.org/journals/ bu17/2013-50-01/S0273-0979-2011-01364-7/S0273-09792011-01364-7.pdf
}

"We invite readers to submit letters to the editor to notices1etters@ams.org.

For permission to reprint this article, please contact: reprint-permission@ams.org.

\section{Why No Permanent Position for Noether at IAS?}

I completely agree with Yvette's comments [above]. Further information can be found in my own review ${ }^{2}$ of her book. One aspect that all such articles seem to skirt around, but should really be explored in much more depth, is why a mathematician of Noether's caliber was not offered a permanent position at IAS or a major research university in the US. She was clearly the equal to if not significantly better than many males from Europe who were offered permanent positions at that time. I doubt it is because of her being either Jewish or a pacifist, and so conclude that this was (primarily) due to her gender. But I would really like to see the details exposed in full.

\section{- Peter Olver \\ University of Minnesota olver@umn.edu}

(Received November 6, 2017)

\section{More on Gravitational Waves}

The issue of August 2017 of the Notices presents introductory articles on gravitational waves, motivated by the detection of oscillatory gravitational signals coming from merging black holes. The texts sketch the history of the theory of gravitational waves. However, they do not mention an important episode: exact solutions of Einstein's equations which are interpreted as plane gravitational waves were discovered already in 1926 by the British physicists O. R. Baldwin and G. B. Jeffery [1]. Their paper disproves the linearization paradigm which says that the full equations are too complicated, only the linearized equations can be fairly handled. The paper also contributes to the discussion of coordinate conditions; such restrictions might be imposed here but need not be. Last but not least, the constructed solutions are free of singularities. The authors apply the usual decomposition of the metrical tensor into $L L, L T$, and $T T$ parts, where $L$ means longitudinal and $T$ means tranversal to the direction of propagation. It turns out that pure $L L$ or $L T$ waves are flat (Minkowskian) while the curvature of $T T$ waves generally does not vanish. Einstein's equations for pure TT waves boil down to a single ordinary differential equation. Vacuum (where there is only gravitation) as well as plane electromagnetic waves fit to plane gravitational waves.

A broader class of gravitaional wave fields was discovered even earlier in 1925, but in a mathematical context, and

\footnotetext{
${ }^{2}$ Bull. Amer. Math. Soc., 2013,www.ams.org/journa1s/ bu11/2013-50-01/S0273-0979-2011-01364-7/S0273-09792011-01364-7.pdt
} 
therefore it remained unnoticed by physicists for a time: H. W. Brinkmann [2] constructed special Lorentzian metrics in the framework of conformal differential geometry which were later named plane-fronted gravitational waves with parallel rays, $p p$-waves for short. The history and prehistory of plane and $p p$-waves is told in the paper [3].

\section{-Rainer Schimming Institute of Mathematics University of Greifswald, Germany}

(Received August 21, 2017)

\section{References}

1) O.R. BALDWIN/G.B. JEFFERY, The relativity theory of plane waves. Proc. Roy. Soc. London A 111 (1926), 95-104.

2) H. W. BRINKMANN, Einstein spaces which are mapped conformally on each other. Math. Annalen 94 (1925), 119-145.

3) R. Schimming, Zur Geschichte der ebenen Gravitationswellen, einer Lösungsklasse der Einsteinschen Gleichungen. NTM Schr. für Geschichte Naturwiss. Tech. Medizin 10 (1973), 21-31.

\section{Contributing Journals to Institutions in Less-Developed Nations}

Recently I decided that it is time to contribute the many back issues of journals (AMS, SIAM, IEEE) that are in my office. The African Institute for Mathematical Sciences (AIMS) seems like a worthy organization, so I wrote to Prof Foupouagnigni of AIMS-Cameroon, offering those journals. He responded:

"Yes, I am interested in the mathematical journal you would like to donate to us. These for sure will be very useful for our students specially during these periods during which we are now gradually shifting to bridge gap between us and industry."

I then wrote to bookdonations@ams.org asking for AMS assistance, saying in part:

"As I am close to Providence I would like to bring them to you (boxed or unboxed, as you prefer) so you can ship them, and so that I can obtain a receipt for tax purposes."

The AMS response came from Kim Kuda, Senior Program Coordinator, Professional Programs Department:

"Our book donation program has a procedure in place where potential schools/universities apply to receive the donations we have posted on our website. Their requests are then reviewed by the Book Donation Steering Committee, and if a match is made, then the donations are shipped directly from the donor to the recipient. We do not accept donations here at our facility. In addition, our program only accepts research books and monographsjournals are not accepted. And finally, we do not supply receipts for tax (or any other) purposes as the donation is not being made to the AMS but to the recipient."

Unsatisfied with this bureaucratic response, I wrote to Catherine Roberts, AMS Executive Director:

"Is the policy enunciated by Kim Kuda written in stone? Frankly it seems rather arbitrary to me. For example, I am sure that there are many others who have journals that they would be happy to contribute, and many institutions in the less-developed world that could make good use of them.
"If it would help I could be convinced to make a contribution to the AMS to partially defray the shipping costs."

Catherine's response:

"Thank you for reaching out to the AMS with your idea to donate your old copies of several journals, including AMS journals, to your contact in Cameroon. Due to the logistics and expenses involved, the policy outlined in Kim's email response to your inquiry does accurately reflect our Society's approach. I am sorry we aren't able to help. Perhaps you could alert Prof Foupouagnigni to the resources that Kim described?"

I strenuously question this policy. It seems ridiculous to me that the AMS is unwilling to assist in giving access to the valuable resource that such journals would provide to both students and faculty in the less-developed world. Per www.ams.org/about-us/about-us the mission of our Society is:

"To further the interests of mathematical research, scholarship and education, serving the national and international community through publications, meetings, advocacy and other programs."

And our goals are:

"1. Promote mathematical research, its communication and uses

2. Encourage and promote the transmission of mathematical understanding and skills

3. Support mathematical education at all levels

4. Advance the status of the profession of mathematics, encouraging and facilitating full participation of all individuals

5. Foster an awareness and appreciation of mathematics and its connections to other disciplines and everyday life"

How does this policy serve our mission? Doesn't affording access to such journals assist with every one of these five goals?

Our website further states:

"The AMS encourages the solicitation and acceptance of gifts for purposes that will help it further and fulfill its mission."

I urge the AMS to reverse this policy and thereby help enable the contribution of journals to mathematical institutions that wish to have them.

-Jim Byrnes President Prometheus Inc., a Woman-Owned Small Business jim@prometheus-us.com

(Received October 6, 2017) 


\section{Mathematics People}

\section{Nunes Receives Freudenthal Award}

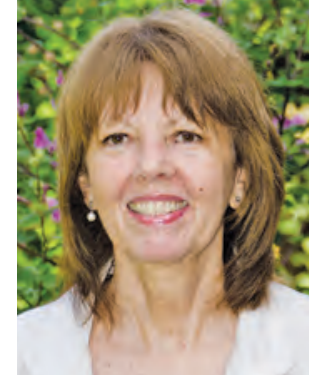

Terezinha Nunes
TEREZINHA NunEs of the University of Oxford has been chosen the recipient of the 2017 Hans Freudenthal Award for her "outstanding contribution to our understanding of mathematical thinking, its origins and development." Her research studies children's mathematical learning in different settings. According to the prize citation, "the results of her numerous, exemplarily designed studies combine into an insightful, consistent, and comprehensive story of the emergence and evolution of mathematical thinking. This constantly developing account has been inspiring the work of mathematics education researchers and informing mathematics teachers' practices all over the world. It has had a major impact on both what we know about children's learning of mathematics and how we know and think about it." Trained as a psychologist, Nunes "has studied children's logical reasoning and its role in the learning of mathematics, as well as problem solving and the way mathematics is being used in science." She has consistently used her research to improve the teaching of mathematics. Nunes tells the Notices: "I feel extremely privileged to have always had support from gifted colleagues in research and my family and friends. This has allowed me to carry on with an academic career and to be a mother, grandmother, and housewife, enjoying playing with children, cooking, sewing, and having fun with friends. I'm just an ordinary person who works very hard."

The Freudenthal Award, given by the International Commission on Mathematical Instruction (ICMI), acknowledges the outstanding contributions of scholars who have initiated new research programs and brought them to fruition over the preceding ten years.

\section{-From an ICMI announcement}

\section{Ball Receives Felix Klein Award}

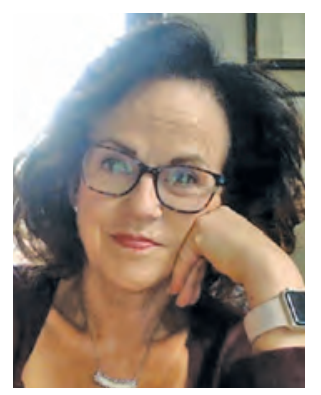

Deborah Loewenberg Ball
DEBORAH LOEWENBERG BALL of the University of Michigan has been named the 2017 recipient of the Felix Klein Prize "in recognition of her outstanding contributions and her leadership role in deepening our understanding of the complexities of teaching mathematics and in improving the practice of teaching and of teacher education." Her research on improving ways in which mathematics teachers support their students' learning has combined research with the practice of teaching through development of innovative teacher preparation programs. Her work led her to develop the theory of MKT, mathematical knowledge for teaching. The theory has been used to develop an instrument for measuring teachers' knowledge of mathematics for teaching. She also established TeachingWorks at the University of Michigan, a national organization to improve the preparation of teachers. Ball began her career as an elementary school teacher and received her $\mathrm{PhD}$ in mathematics education from Michigan State University (1988), which she then joined as a faculty member. In 1996 she moved to the University of Michigan, where she developed the mathematics education group, and has remained there, serving for more than ten years as dean of the School of Education. She is a member of the American Academy of Arts and Sciences and a Fellow of the AMS.

Ball tells the Notices: "I majored in French as an undergraduate. I decorated wedding and birthday cakes for a living at one point. I was also a good cocktail waitress in college, [which] did involve a lot of mathematical reasoning."

The Felix Klein Award is given by the International Commission on Mathematical Instruction (ICMI) for lifetime achievement in mathematics education research, especially introducing new ideas, perspectives, and critical reflections. 


\section{Prizes of the Mathematical Society of Japan}

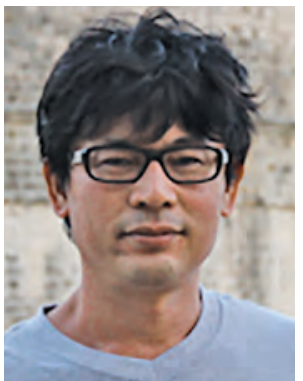

Tomoyuki

Arakawa
The Mathematical Society of Japan (MSJ) has announced its Autumn Prize and several other prizes for 2017.

TOMOYUKI ARAKAwA of Kyoto University has been awarded the 2017 Autumn Prize for his outstanding contributions to representation theory of $W$-algebras. The Spring Prize and the Autumn Prize are the most prestigious prizes awarded by the MSJ to its members.

The 2017 Analysis Prizes have been awarded to the following: TETSUTARO SHIBATA (Hiroshima University) for work on the asymptotic analysis of eigenvalue problems for nonlinear elliptic equations and analysis of inverse bifurcation problems; MASAYOSHI TAKEDA (Tohoku University) for work on stochastic analysis of symmetric Markov processes and its applications; and YosHITSUGU TAKEI (Doshisha University) for work on asymptotic analysis of linear and nonlinear differential equations with exact WKB analysis.

The 2017 Geometry Prizes were awarded to OSAMU KOBAYASHI (Osaka University) for various far-seeing contributions in differential geometry and to MAKOTO SAKUMA (Hiroshima University) for a series of works on knots and hyperbolic geometry.

The 2017 Takebe Katahiro Prizes were awarded to the following: TsUKASA IwABUCHI (Tohoku University) for work on the well-posedness for nonlinear evolution equations by the method of real analysis; SEIICHIRO KUSUOKA (Okayama University) for new developments of study on fundamental solutions via stochastic calculus; HARUYA MizUTANI (Osaka University) for work on Strichartz estimates for Schrödinger equations; and TAKAYUKI OKUDA (Hiroshima University) for work on discontinuous groups and combinatorics of homogeneous spaces. The Takebe Katahiro Prize is awarded to young researchers who have obtained outstanding results.

The Takebe Katahiro Prizes for Encouragement of Young Researchers were awarded to the following: YUKI ARANO (Kyoto University) for studies on operator algebraic quantum groups; MASATO HosHINO (Waseda University) for studies on ill-posed stochastic partial differential equations; SACHIKO ISHIDA (Chiba University) for studies on quasilinear degenerate Keller-Segel systems; AKIHIRO KANEMITSU (University of Tokyo) for contributions to Campana-Peternell conjecture and Mukai's problem on Fano manifolds; TATSUYA MIURA (University of Tokyo) for mathematical analysis on effects of bending, adhesion and tension energy to shapes; and TAKAMICHI SANO (Osaka City University) for studies on the equivariant Tamagawa number conjecture and Euler systems, especially RubinStark elements. The Prizes are intended for young math-

ematicians who are deemed to have begun promising careers in research by obtaining significant results.

-Elaine Kehoe

\section{Prizes of the New Zealand Math Society}

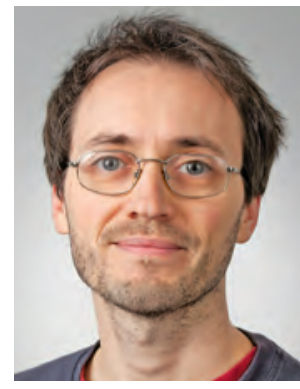

Igor Klep

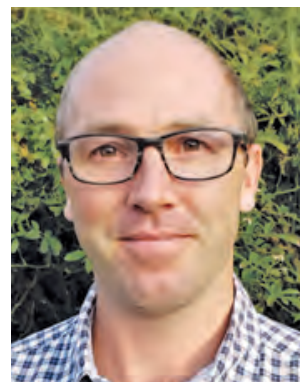

Brendan Creutz

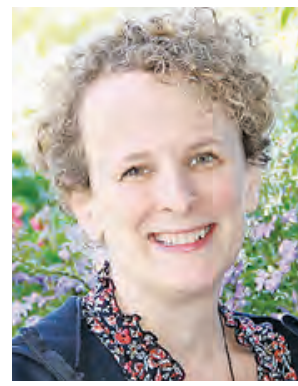

Lisa Orloff Clark
The New Zealand Mathematical Society (NZMS) has announced several awards for 2017.

IGOR KLEP of the University of Auckland is the recipient of the Research Award "for deep and fundamental advances in real algebraic geometry and its application to diverse fields, including operator theory, optimization, free analysis, convexity, and von Neumann algebras." The prize is awarded for mathematical research by a mathematician in New Zealand that has been published in books or journals within the previous five calendar years.

BRENDAN CREUTZ of the University of Canterbury received the Early Career Research Award "for his outstanding work on local-global questions on diophantine equations, in particular his resolution of a 50-year-old question of Cassels and the development of novel computational techniques to study the arithmetic of algebraic curves and surfaces." The prize is given for excellent research carried out by early-career New Zealand mathematicians within seven years of receipt of the $\mathrm{PhD}$.

LISA ORLOFF ClARK was named the recipient of the Kalman Prize for Best Paper for Jonathan H. Brown, Lisa Orloff Clark, Cynthia Farthing, and Aidan Sims, "Simplicity of algebras associated to etale groupoids," Semigroup Forum 88 (2014). Clark tells the Notices: "I love to dance. I have taken classes in numerous styles over the past twenty-five years and enjoy every opportunity to 'cut a rug.' My current outlet is Cuban salsa, which is a lot of fun."

The Kalman Prize is given for an outstanding and innovative piece of research in the mathematical sciences published by a member or members of the NZMS.

-From an NZMS announcement 


\section{Wallenberg Fellowships Awarded}

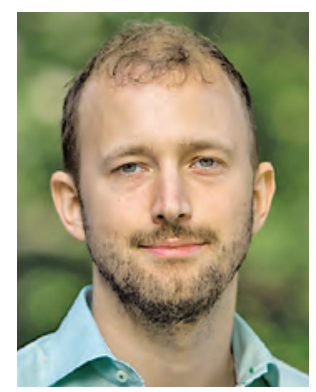

Dan Petersen

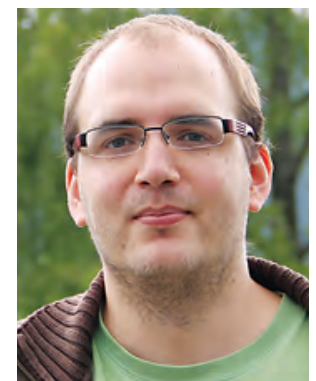

Karim Adiprasito
Two mathematical scientists have been awarded fellowships by the Wallenberg Academy. DAN PETERSEN of the University of Copenhagen was honored for his work in algebraic geometry, particularly in his specialty, the theory of moduli space, which links to number theory and string theory. Petersen will use his fellowship to work at Stockholm University. He tells the Notices: "I am currently on paternity leave with my second child. In my rapidly shrinking free time I enjoy cooking and reading comic books." KARIM ADIPRASITO of Hebrew University of Jerusalem and Leipzig University was selected as a fellow for his work that "unites three important areas" of mathematics: combinatorics, geometry, and algebra. He has been studying new mathematical methods to study the ways that discrete objects behave as continuous. According to the citation, "a breakthrough for these new methods was his solution of a more than 200-year-old problem in which he, in a discrete context, used partial differential equations that usually describe continuous processes." Adiprasito received the European Prize in Combinatorics in 2015. He will use his fellowship to work at KTH Royal Institute of Technology in Stockholm. He tells the Notices: "I am part Indonesian, and my last name is a mix of Sanskrit and Javanese. I planned to be a chef after high school."

Wallenberg Fellows receive five-year grants of 1 to 2 million Swedish krona (approximately US\$119,000-238,000) per year, depending on the field. The program was established by the Knut and Alice Wallenberg Foundation in close cooperation with five learned academies and sixteen Swedish universities to give the most promising young researchers a work situation that enables them to focus on their projects and address difficult research questions over an extended period of time.

\section{-From a Wallenberg Academy announcement}

\section{AAAS Fellows}

The American Association for the Advancement of Science (AAAS) has elected its new fellows for 2017.

The new Fellows of the Section on Mathematics are:

- EDWARD F. AboufADEL, Grand Valley State University

- Qiang Du, Columbia University

- Joceline LEGA, University of Arizona

- W. JAMES (JIM) LEWIS, University of Nebraska-Lincoln and National Science Foundation

- Peter MARch, Rutgers, The State University of New Jersey

- Panatiotis E. Souganidis, University of Chicago

The new Fellows of the Section on Statistics are:

- ERIC D. KolaczyK, Boston University

- Hongzhe LI, University of Pennsylvania Perelman School of Medicine

- Runze Li, Pennsylvania State University

- Bhramar MukHERJEe, University of Michigan

- Douglas G. SIMPSON, University of Illinois at UrbanaChampaign

- Rochelle E. Tractenberg, Georgetown University

-From an AAAS announcement

\section{Rhodes Scholars Announced}

The Rhodes Trust has announced the names of thirty-two American scholars chosen as Rhodes Scholars for 2018. Following are the names and brief biographies of the scholars whose work involves the mathematical sciences.

NATHAN R. BERMEL of Chestnut Hill, Massachusetts, is a senior at the United States Naval Academy, where he majors in quantitative economics and political science. He ranks first in the Academic Order of Merit and second in the Military Order of Merit among 1,088 of his fellow midshipmen. He has interned for the Bureau of Energy Resources at the State Department, where he conducted analyses of the oil market in Iraq and Syria for the department's counter-ISIL team. In the summer of 2017, he traveled to the Kwajalein Atoll to research the effects of climate change on the Republic of the Marshall Islands. He was a member of the Naval Academy's two-time National Championship Club Lacrosse team. At Oxford, he will read for an MPP.

NOAH V. BARBIERI of Belden, Mississippi, is a senior and Truman Scholar at Millsaps College, where he is pursuing a BSc in economics, mathematics, and philosophy. He has completed an honors thesis in philosophy and another in economics. His research has probed the antecedents of income inequality and explored the factors that affect labor force participation among prime age males. He also serves as the student body president and was awarded the Student Body Leader of the Year Award in 2017 as voted on by his peers. He aspires to a career in public service. At Oxford, he will read for an MPhil in economics. 


\section{NEWS}

HAROLD XAVier Gonzalez of Houston, Texas, is a senior concentrating in mathematics at Harvard College. His research has focused on the unexpected connection, ultimately provided by techniques from theoretical physics, between modular forms and finite symmetry groups. Gonzalez is a four-year varsity tennis player. He presented a paper at the Annual Undergraduate Shakespeare Conference, is a peer advising fellow, and volunteered at a homeless shelter. At Cambridge University he took courses in Shakespeare and also in gravitational waves. His professional goals are to promote the public interest in math and to use math and its methods to promote the public interest. He will do the MSc by research in mathematics at Oxford.

SEAN P. ReILly of San Jose, California, graduated from Santa Clara University in 2016 with a BSc in environmental science and biology. He wrote his honors thesis on the influence of the Santa Cruz Mountains on precipitation from a landfalling atmospheric river. The president of the SCU Green Club and cofounder of Fossil Free SCU, he successfully collaborated with university administrators to set up a sustainable investment fund. He was awarded a Fulbright Scholarship in 2017 to lead a community-based participatory research project with the Mandingalbay Yidinji aboriginal people to manage the pond apple, a formidable bioinvader valued by the community as a food source. He is also a rock climber, pianist, guitar player, and a gold-medal winner in the North American Roller Hockey Championship. At Oxford, he will read for the MSc in mathematical modeling and scientific computing.

-From a Rhodes Trust announcement

\section{Tsitsiklis Receives IEEE Award}

JoHN N. TSITSIKLIS of the Massachusetts Institute of Technology has received the IEEE Award in Control Systems "for contributions to the theory and application of optimization in large dynamic and distributed systems." He received a BS in mathematics in 1980 and his $\mathrm{PhD}$ in electrical engineering in 1984, both from MIT.

\section{-From an IEEE announcement}

\section{AMS Members Elected Fellows of ACM}

Four members of the AMS have been elected Fellows of the Association for Computing Machinery: CLIFFORD LYNCH of the Coalition for Networked Information; BALAJI PRABHAKAR of Stanford University; MICHAEL SIPSER of the Massachusetts Institute of Technology; and ALEXANDER VARDY of the University of California San Diego.

Sipser is also a Fellow of the AMS. The ACM Fellows Program recognizes exceptional contributions of leading members in the computer field.

-From an ACM announcement

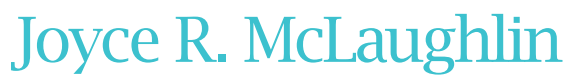

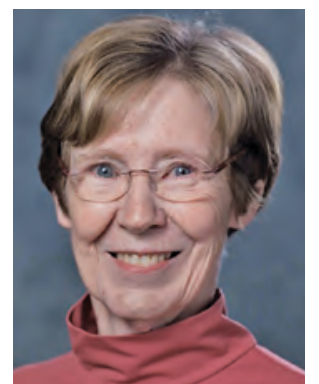

Joyce $\mathbf{R}$. McLaughlin
Joyce R. McLaughlin, Ford Foundation Professor of Mathematical Sciences at Rensselaer Polytechnic Institute (RPI), passed away on October 23,2017 . She was also the founding director of the Inverse Problems Center at RPI (IPRPI).

Her main area of research interest was in nonlinear analysis as applied to parameter identification in inverse problems. Applications of her research included medical imaging, ocean acoustics, and inverse problems in vibrating systems. She gave an invited lecture at the International Congress of Mathematicians in 1994 and a CBMS lecture series in 2001. She was also known for her work in biomechanical imaging of tissue.

She received her $\mathrm{PhD}$ in applied mathematics from the University of California Riverside. She was named an inaugural fellow of the AMS in 2012. She was an inaugural fellow of the Society for Industrial and Applied Mathematics (SIAM) and was awarded the Kovalevsky Lectureship and Prize of SIAM and the Association for Women in Mathematics in 2004. She was the first woman chaired professor at RPI and was also an airplane pilot.

-Elaine Kehoe

\section{Photo Credits}

Photo of Terezinha Nunes courtesy of Terezinha Nunes.

Photo of Deborah Loewenberg Ball courtesy of Deborah Loewenberg Ball.

Photo of Tomoyuki Arakawa courtesy of Tomoyuki Arakawa. Photo of Igor Klep courtesy of Brian Donovan, The University of Auckland.

Photo of Brendan Creutz by Nika Creutz.

Photo of Lisa Orloff Clark courtesy of Archives of the Mathematisches Forschungsinstitut Oberwolfach.

Photo of Dan Petersen by Markus Marcetic, (c) Knut och Alice Wallenbergs Stiftelse Kungl. Vetenskapsakademien.

Photo of Karim Adiprasito courtesy of Archives of the Mathematisches Forschungsinstitut Oberwolfach.

Photo of Joyce R. McLaughlin courtesy of Rensselaer Polytechnic Institute. 


\title{
Herbert Busemann
}

\author{
Athanase Papadopoulos
}

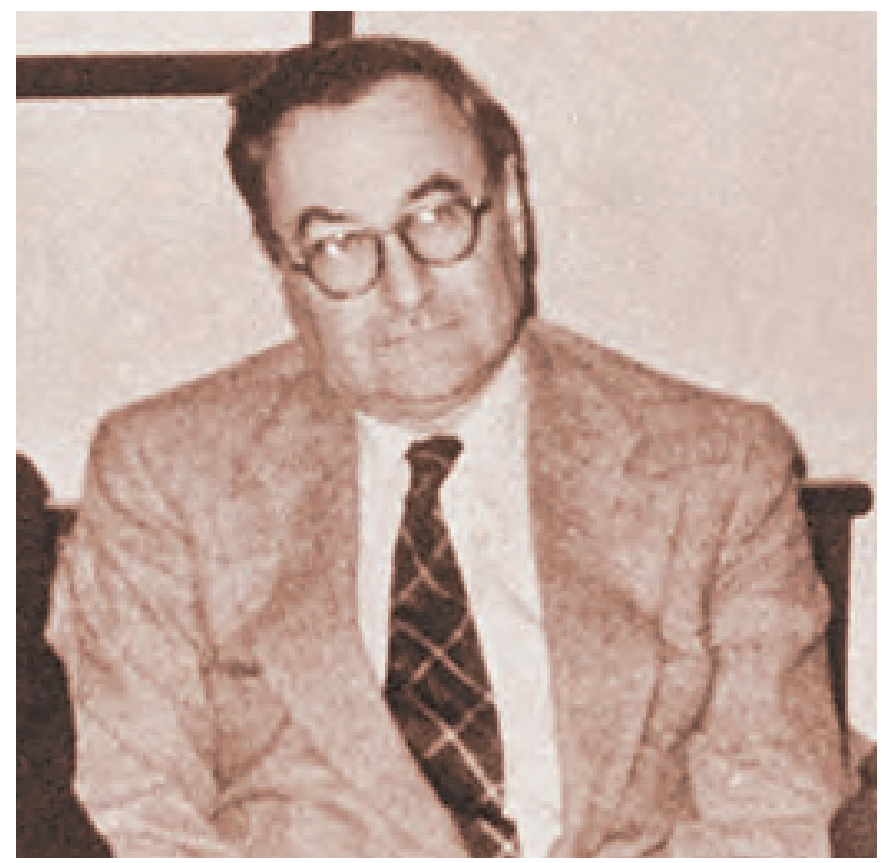

Herbert Busemann, 1954

EDITOR'S NOTE. Athanase Papadopoulos has kindly let us publish these excerpts from his introductory material $^{1}$ to Herbert Busemann: Selected Works I and $I^{2}$. Busemann was deeply involved in fundamental questions of convexity and is the main founder of metric geometry as we intend it today. He was a member of the Royal Danish Academy, a winner of the Lobachevsky Medal (1985), president of the California chapter of the Mathematical Association of America, a member of the council of the American Mathematical Society, and an accomplished linguist and artist.

Athanase Papadopoulos is director of research at the Institute of Advanced Mathematical Research at the University of Strasbourg. His email address is athanase.papadopou1os@math. unistra.fr.

For permission to reprint this article, please contact: reprint-permission@ams.org.

DOI: http://dx.doi.org/10.1090/noti1651

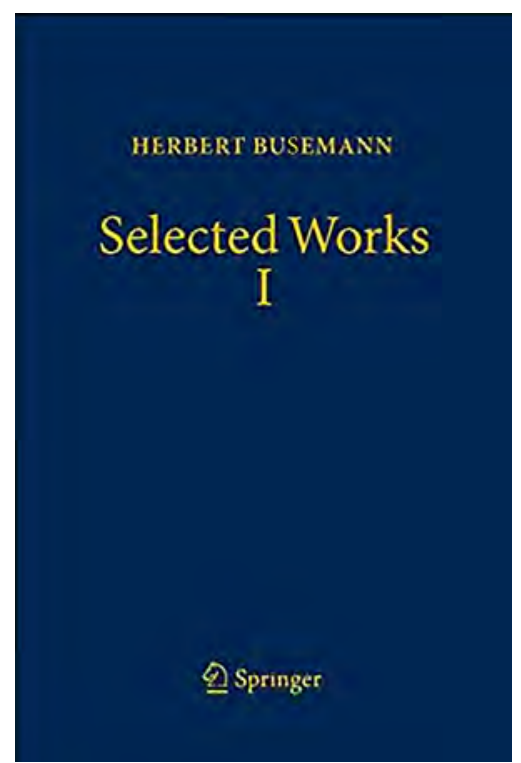

Herbert Busemann, Selected Works in two volumes by Herbert Busemann; Athanase Papadopoulos, ed.

Springer, 2018

908 and 842 pages.

Busemann recounts the following:

Officially, I took my degree with Courant. This was only officially, in the sense that I was really inspired by Paul Alexandroff, who visited Göttingen regularly. He gave me the idea of the subject of the thesis. I wrote it, but of course he could not be the official reviewer of my thesis, so he was my co-referee. [...] I must say that my thesis was partly in protest against Courant [...] I went away to Rome. I was angry with Courant, and I wrote my thesis on my own....

In a letter dated August 8, 1930, Courant informs Busemann that his "geometric work" done in Rome is sufficient

$\overline{\text { arXiv:1710.05591v1(16 Oct 2017) }}$

${ }^{2}$ Herbert Busemann, Selected Works in two volumes (A. Papadopoulos, ed.), Springer Verlag, 2018. 
for a "reasonable" dissertation. The final dissertation was submitted in December 1930....

The doctoral degree that Busemann obtained in 1931 would have led him in principle to a position of lecturer, but practically it did not. He recounts:

Normally I would become an assistant but this was of course the depression time. Since my father had money and Courant knew it, he asked my father whether he wouldn't support me instead of having me become an assistant. This would make it possible for some other youngster to become a mathematician. That fact did me a lot of harm later on, in the sense that the German government after the Second World War tried to make good, but in my case they refused, because I had never had a paid position. If I had a paid position I would have had a pension.

Courant, as the director of the Mathematics Institute in Göttingen, told Busemann that they had run over their funds and asked him to make it possible to meet his father in order to get some financial support from him for the institute. The two men met. Busemann says: "How they settled this situation I don't know and I thought I shouldn't ask." Reid reports on this in her book on Hilbert $^{3}$ (p. 132):

During the increasingly hard times, a number of informal assistantships came into being at the institute in addition to the official ones funded by the government. Often duties were vague or non-existent. Courant once gave a student a stipend because he thought the young man was on the verge of a nervous breakdown and needed a skiing vacation. He also contrived to have some students work without pay. One of them was Busemann.

Busemann adds, concerning his former advisor:

Courant was rather reactionary in his mathematical outlook, and so he prevented many things which should not have been prevented. In Göttingen, he constantly tried to prevent the concept of Lebesgue integral. This has in the meantime conquered the whole world. He didn't see the importance of many things of modern mathematics. He had no relations at all with algebraic geometry. ... The Russians had played quite a role in Göttingen. I believe that I was the only one who was directly inspired by them. But their course was very popular. They filled a gap. They were familiar with certain modern tendencies that were not represented in Göttingen, e.g. topology.

His first permanent position was at the Illinois Institute of Technology in Chicago, a position which he described as a "horrible permanent job." He recalls that this was a

$\overline{{ }^{3} \text { C. Reid, Hilbert, Springer Verlag, Berlin-New York, } 1970 .}$ period where "everybody was looking for jobs, and one had to take whatever." He says he spent "five miserable years" in Chicago, from 1940 to 1945. He adds:

The head of the department made it difficult. He did not like foreigners in the first place. He belonged to those people who had done a couple of good things when they were quite young and he was against anyone who was too active mathematically. On the other hand the administration forced him to take good people, and he resented them.

In 1945, Busemann was appointed Assistant Professor at Smith College in Northampton.

Busemann stayed in contact with Courant, and the two men had a regular correspondence, but essentially on practical matters. Talking about the institute that Courant founded later in New York, Busemann says: "In America, Courant tried to do again what he did before in Göttingen. ...His institute is excellent but very one-sided too. The mathematics represented there goes all, or most of it, in one direction."

In 1947, Busemann was appointed professor at the University of Southern California, and he spent there the rest of his career. In 1964, he was made distinguished professor.

At USC, Busemann worked in relative isolation, and practically his only collaborators were his $\mathrm{PhD}$ students. His work started to be recognized only in the 1980s, when metric geometry was revived in the West, especially by M. Gromov, and when the methods of synthetic global geometry were introduced in the study of geodesic metric spaces. W. P. Thurston, in his approach to geometry, also started from basic principles. Before that, Busemann's work was only appreciated in the Soviet Union, where A. D. Alexandrov founded an important school on the subject, with a large number of collaborators and students. Alexandrov, like Busemann, was only interested in the most basic notions of geometry. Classical problems of convexity, isoperimetry, and isoepiphany became the forefront of research, and classical projective geometry took its revenge upon a certain Riemannian geometry based on linear algebra and tensor calculus in tangent spaces. In some sense, it was a return to Euclid and Archimedes. In a tribute to Alexandrov's memory, S. S. Kutateladze writes:

"Alexandroff contributed to mathematics under the slogan: 'Retreat to Euclid.' He remarked that the pathos of contemporary mathematics is the return to Ancient Greece."

Busemann's work is profound. He was capable of formulating problems and working on them, without relying on the trends that were fashionable in his time. In an article that appeared in the Los Angeles Times on June 14, 1985, ${ }^{4}$ on the occasion of the attribution of the Lobachevsky prize to Busemann, the author reports the following: "Few mathematicians ever make it into public consciousness, but

\footnotetext{
${ }^{4}$ L. Dembart, An unsung geometer keeps his own plane,
} Los Angeles Times, June 14, 1985. 
Busemann has had a hard time even within his own field, in part, at least, because he never followed the crowd."

The journalist quotes Busemann saying: "If I have a merit, it is that I am not influenced by what other people do." He then quotes Bob Brooks, who was Busemann's colleague at USC:

Tastes change a lot and interests change a lot in the space of five years. But there are people who aren't so interested in keeping up with today's fads. Busemann is very definitely in that category.

We also read in the same article:

Busemann characterizes his basic mathematical approach this way: "Any apparently difficult problem can be done with very simple methods. This is the property of many of my things. I see a simple geometric reason which others have overlooked."

Talking about the Geometry of Geodesics for which the Lobachevsky Medal was attributed, Busemann declares, in the Los Angeles Times article, that the approach is more important than the results: "The emphasis is more on the radically new approach than on the individual problem."

Busemann retired in 1970. In 1971, he received from USC an honorary degree of Doctor of Laws. He was also a linguist. He spoke several languages, including, besides German and English, French, Spanish, Italian, Russian, and Danish. He wrote papers or translated articles and monographs from all these languages. Busemann published in the Mathematical Reviews a large number of reports on articles written in Russian. He could also read Arabic, Latin, Greek and Swedish. In the article in the Los Angeles Times mentioned above, the journalist quotes Busemann: "Every two years I read the Odyssey, I like it so much. And Plato." Busemann was also a painter. In the same article in the Los Angeles Times, we read: "Despite a lifelong desire to paint, Busemann never took it up, fearing that it would divert him from the arduous work of mathematics. But retirement freed him, and he built a studio in his home that is now chockablock with dozens of large canvases painted in vibrantly colored geometrical designs. His mathematical vision carried over into art."

Busemann got married in 1939. Little is known about his personal life. In a recent letter to the author of the present article, Peter Woo, who was a student of Busemann, writes:

Busemann had a funny way of writing on the blackboard. Some important theorem, he would write in big letters, from left edge to right edge, some 15 feet wide. Then he would scribble the proof underneath, and say, "See how easy it is," and then erase his proof. We had to beg him not to wipe away until we finished copying it. He would give us a puzzled look, as if we were wasting his time.
He wanted me to discover theorems about new geometrical spaces with the rule : $A B+B C$ $\leq \mathrm{AC}$ unless A, B, C lie on a "geodesic curve." First I found this funny. Then I began to make conjectures, and he encouraged me to prove them, first in some particular cases, then in more and more general cases. At some point he said, "You have done enough for the $\mathrm{PhD}$ dissertation."

He often took us to his home. Together with three other PhD students, we used go to his house one afternoon per week. Each of us was supposed to present some theorem or unsolved problem, on a blackboard hanging on the wall at his backyard. He would make some remarks on what direction we should turn to, some easy special cases we had to study first, etc. After that, he would invite us into his house, to have a piece of pastry, and tea or coffee. He was always positive. He did not tell us much about his life. He knew a lot about history of mathematics, and this tied us with the European cultural heritage. He liked plants. He had a cactus garden where we loved a little thing about 9" tall, 4" wide, like a ridged dark green okra, with much white hair spreading from the top all around. He called it "the Old Man." We loved it. He had no children, he loved us almost like his children.

\section{Image Credits}

Photo of Herbert Busemann courtesy Author: Konrad Jacobs. Source: Archives of the Mathematisches Forschungsinstitut Oberwolfach. Printed photo is a crop of the original.

Image of Herbert Busemann, Selected Works in two volumes (A. Papadopoulos, ed.), courtesy of Springer Verlag, 2018. Photo of Athanase Papadopoulos courtesy of the author.

\section{ABOUT THE AUTHOR}

Athanase Papadopoulos studied engineering at Ecole Centrale de Paris, then obtained a PhD in mathematics at Université de Paris-Sud. Since then, he has been a research fellow at the French CNRS. He edited the complete musical works of Euler and Menelaus' Spherics. He has four children, all of them musicians.

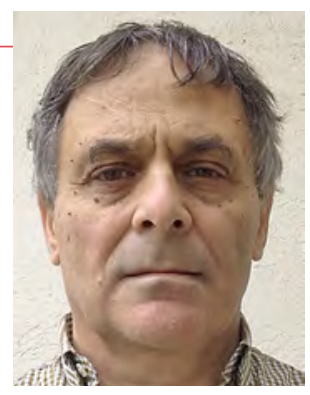

Athanase Papadopoulos 


\section{Mathematics Opportunities}

\section{Listings for upcoming math opportunities to appear in Notices may be submitted to notices@ams.org.}

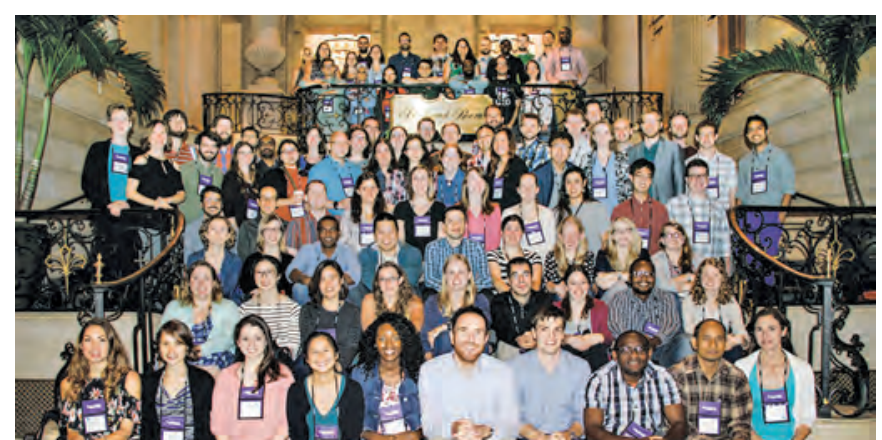

2017 Project NExT Fellows (Blue'1 7 Cohorts) in Chicago.

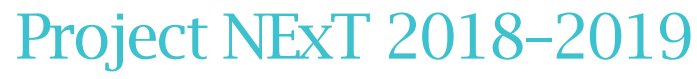

Project NExT (New Experiences in Teaching) is a yearlong professional development program of the Mathematical Association of America (MAA) for new or recent PhDs in the mathematical sciences. The program is designed to connect new faculty with master teachers and leaders in the mathematics community and address the three main aspects of an academic career: teaching, research, and service. The program welcomes and encourages applications from new and recent PhDs in postdoctoral, tenure-track, and visiting positions. The deadline for applications for the 2018 cohort of MAA Project NExT Fellows is April 15, 2018. For applications and further information, see www.maa.org/programs/faculty-and-departments/ project-next.

-From an MAA announcement

\section{Graham Wright Award: Call for Nominations}

The Graham Wright Award for Distinguished Service of the Canadian Mathematical Society (CMS) recognizes individuals who have made sustained and significant contributions to the Canadian mathematical community and, in particular, to the CMS. The deadline for nominations is March 31, 2018. See cms . math . ca/Prizes/dis-nom.

-From a CMS announcement

\section{Aisenstadt Prize: Call for Nominations}

The Centre de Recherches Mathématiques (CRM) seeks nominations for the André Aisenstadt Mathematics Prize, which recognizes outstanding research by a young Canadian mathematician. The deadline for nominations is March 1, 2018. See www. crm . umontreal.ca/prix/prix AndreAisenstadt/prix_attributionAA_an.shtm1.

\section{-From a CRM announcement}

\section{Diversity Conference in the Mathematical Sciences}

The Infinite Possibilities Conference (IPC), a program of Building Diversity in Science (BDIS), is a national conference designed to promote, educate, encourage, and support women of color interested in mathematics and statistics, as a step toward addressing the underrepresentation of African Americans, Latinas, Native Americans, and Pacific Islanders in these fields. The conference will be held April 14-15, 2018, at Howard University. See mathinstitutes.org/diversity for more information.

\section{-From an MSRI announcement}

\section{TWAS Prizes: Call for Nominations}

The World Academy of Sciences (TWAS) awards annual prizes to scientists who have been living and working in a developing country for at least ten years. The deadline for nominations is March 15, 2018. See twas . org /opportunity/twas-prizes.

-From a TWAS announcement

Photo Credit

Photo of ProjNExT Blue 2017 by MAA staff, courtesy of David Kung. 


\section{Join the AMS... \\ or renew your membership today}
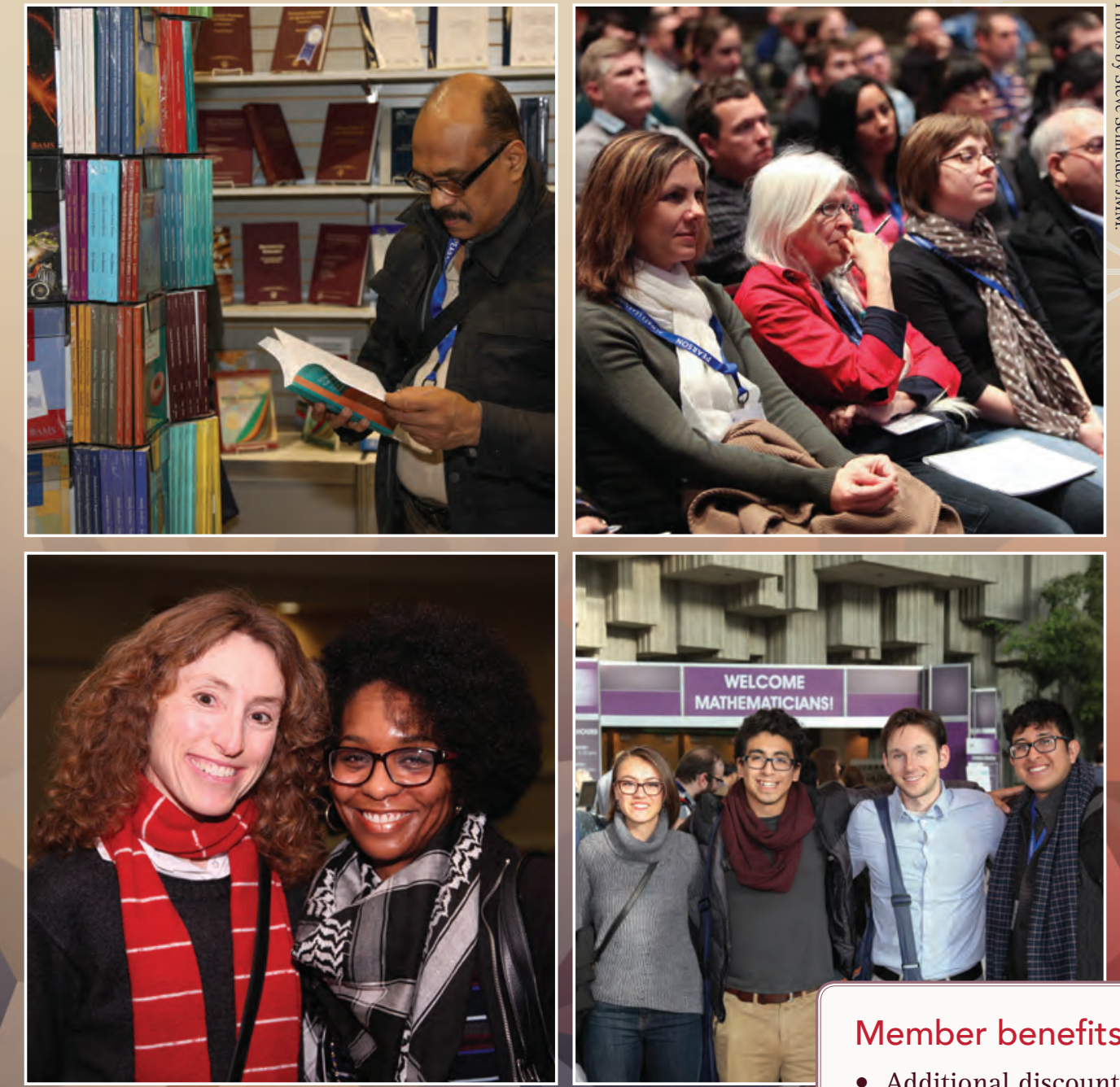

Member benefits:

- Additional discounts on books purchased at meetings

- Subscriptions to Notices and Bulletin

- Discounted registration for world-class meetings and conferences 
Sixty-three mathematical scientists from around the world have been named Fellows of the American Mathematical Society (AMS) for 2018.

The Fellows of the American Mathematical Society program recognizes members who have made outstanding contributions to the creation, exposition, advancement, communication, and utilization of mathematics. Among the goals of the program are to create an enlarged class of mathematicians recognized by their peers as distinguished for their contributions to the profession and to honor excellence.

The 2018 class of Fellows was honored at a dessert reception held during the Joint Mathematics Meetings in San Diego, California. Names of the individuals who are in this year's class, their institutions, and citations appear below.

The nomination period for Fellows is open each year from February 1 to March 31. For additional information about the Fellows program, as well as instructions for making nominations, visit the web page www.ams.org/profession/ ams-fellows.

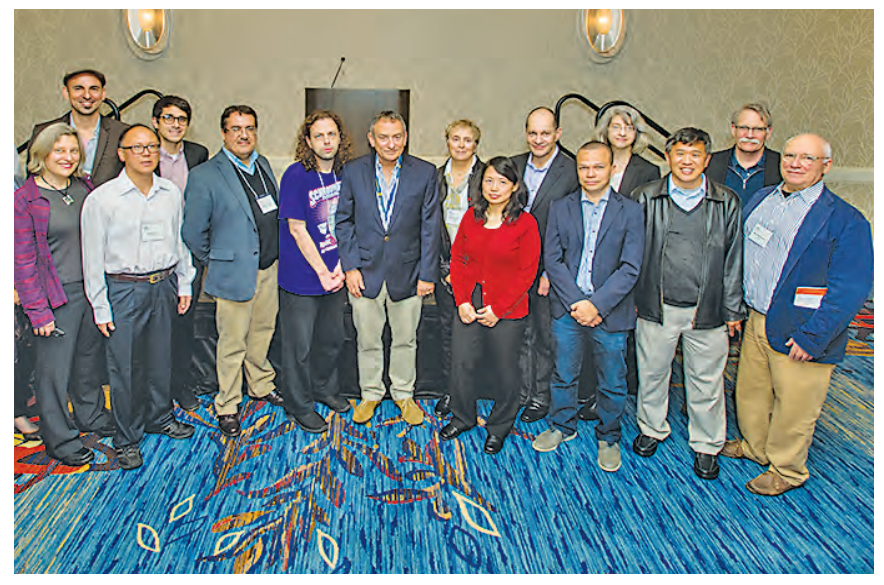

Members of the 2018 Class of Fellows of the AMS who attended the reception at the JMM in San Diego.

Mohammed Abouzaid, Columbia University

For contributions to symplectic geometry, homological mirror symmetry, and service to the profession.

Daniel D. Anderson, University of Iowa For contributions to commutative algebra and its exposition, service to the mathematical community, and mentoring.

Federico Ardila, San Francisco State University For contributions to combinatorics and service to the profession.

Hélène Barcelo, Mathematical Sciences Research Institute For contributions to combinatorics and service to the profession.

Estelle Basor, American Institute of Mathematics For contributions to analysis and for service to the mathematical community.
Kai Behrend, University of British Columbia For contributions to algebraic geometry.

Michele Benzi, Emory University

For contributions in numerical linear algebra, exposition, and service to the profession.

Don Blasius, University of California, Los Angeles For contributions to arithmetic algebraic geometry and representation theory.

Lev Borisov, Rutgers The State University of New Jersey New Brunswick

For contributions to algebraic geometry and mirror symmetry.

Aldridge Bousfield, University of Illinois at Chicago For contributions to homotopy theory and for exposition.

Steven Bradlow, University of Illinois, Urbana-Champaign For contributions to geometric analysis and moduli theory, and service to the mathematical community.

Emmanuel Candès, Stanford University

For contributions to the field of compressed sensing, and to multiscale analysis, statistics, and matrix completion.

Izzet Coskun, University of Illinois at Chicago For contributions to algebraic geometry, and for mentoring.

James Davis, Indiana University, Bloomington For contributions to geometric topology especially through surgery theory, and for exposition.

Peter Ebenfelt, University of California, San Diego For contributions to CR geometry, complex geometry, and several complex variables, and for mentoring and professional service. 
Joan Ferrini-Mundy, National Science Foundation For contributions to mathematics education, and to the development of educational policy and initiatives.

Antonella Grassi, University of Pennsylvania For contributions to algebraic geometry and mathematical physics, and for leadership in mentoring programs.

Jesper Grodal, University of Copenhagen For contributions to algebraic topology, representation theory and their interactions, and for service to the profession.

Cristian E. Gutierrez, Temple University For contributions to nonlinear partial differential equations and its applications to geometric optics, and mentoring.

David A. Hoffman, Stanford University For contributions to differential geometry, particularly minimal surface theory, and for pioneering the use of computer graphics as an aid to research.

William A. Howard, University of Illinois at Chicago For contributions to proof theory and constructive foundations of mathematics.

Alexandru Ionescu, Princeton University

For contributions to harmonic analysis on symmetric spaces, discrete analogs of singular Radon transforms, and the mathematics of general relativity.

Thomas Kailath, Stanford University

For contributions to information theory and related areas, and for applications.

Sergiu Klainerman, Princeton University

For contributions to partial differential equations, to the mathematical theory of general relativity, and mentoring.

Nicholas J. Kuhn, University of Virginia

For contributions to homotopy theory, group cohomology and representation theory.

Igor Kukavica, University of Southern California For contributions to nonlinear partial differential equations, mentoring, and service to the profession.

Max Lieblich, University of Washington

For contributions to algebraic geometry and for service to the mathematical community.

Ivan Losev, Northeastern University

For contributions to geometric representation theory, noncommutative algebra, and the theory of categorification.

Guozhen Lu, University of Connecticut, Storrs

For contributions to harmonic analysis and partial differential equations, and for service to the mathematical community.

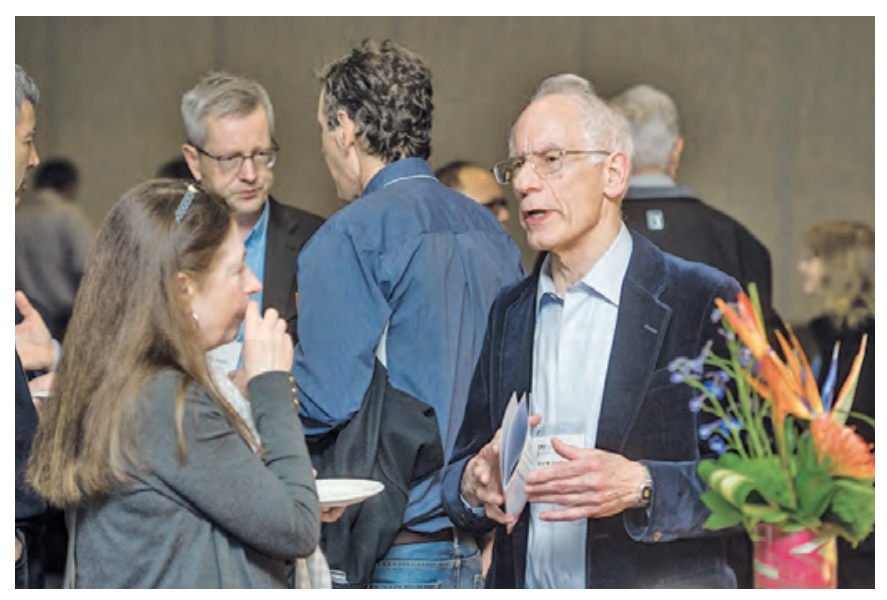

AMS Secretary Carla D. Savage and former AMS President Eric Friedlander.

Feng Luo, Rutgers The State University of New Jersey New Brunswick

For contributions to Teichmueller theory, conformal geometry, hyperbolic geometry, 3-manifolds, combinatorial topology and computer graphics.

Brian Marcus, University of British Columbia For contributions to dynamical systems, symbolic dynamics and applications to data storage problems, and service to the profession.

Fernando Codá Marques, Princeton University For contributions to geometric analysis.

Stephen D. Miller, Rutgers The State University of New Jersey New Brunswick

For contributions to analytic number theory, cryptography, and theoretical physics.

Detlef Müller, Christian-Albrechts-Universität zu Kiel For contributions to harmonic analysis and partial differential equations.

James R. Munkres, Massachusetts Institute of Technology For contributions to algebraic topology, and for exposition.

Aaron Naber, Northwestern University For contributions to differential geometry, particularly to manifolds with bounds on Ricci curvature.

Melvyn B. Nathanson, The City University of New York, The Graduate Center and Lehman College

For contributions to the development of additive combinatorics and service to the profession.

Lei Ni, University of California, San Diego

For contributions to geometric analysis, particularly to Ricci flow, and for mentorship. 


\section{FROM THE AMS SECRETARY}

John Pardon, Princeton University

For contributions to knot distortion, the Hilbert-Smith conjecture, and pseudo-holomorphic curves.

Peter Polacik, University of Minnesota-Twin Cities For contributions to analysis of partial differential equations and infinite-dimensional dynamical systems.

Mason A. Porter, University of California, Los Angeles For contributions to nonlinear and complex systems, and to the emerging field of network science.

Rachel Justine Pries, Colorado State University For contributions to arithmetic geometry, and for service to the mathematical community.

Andrew Putman, University of Notre Dame For contributions to combinatorial and geometric group theory and topology.

Eric M. Rains, California Institute of Technology For contributions to quantum information theory and coding theory, the theory of random matrices, the study of special functions, non-commutative geometry and number theory.

Kavita Ramanan, Brown University

For contributions to the theory and applications of stochastic processes.

Peter Sarnak, Princeton University and The Institute for Advanced Study

For contributions to number theory and analysis.

Larry L. Schumaker, Vanderbilt University

For contributions to spline theory, approximation theory, and numerical analysis, and for service to the profession.

Albert Schwarz, University of California, Davis For contributions to mathematical physics.

Timo Seppäläinen, University of Wisconsin, Madison For contributions to probability.

Roger R. Smith, Texas A\&M University

For contributions to operator theory and operator algebras.

Kannan Soundararajan, Stanford University For contributions to analytic number theory.

Hema Srinivasan, University of Missouri-Columbia For contributions to algebra and algebraic geometry, mentoring, and service to the mathematical community.

Ed Swartz, Cornell University

For contributions to topological and geometric combinatorics.
Simon Tavaré, University of Cambridge

For contributions to population genetics and computational biology, and for service to the profession.

Joseph M. Teran, University of California, Los Angeles For contributions to numerical methods for partial differential equations in classical physics with modern applications.

Richard P. Thomas, Imperial College

For contributions to algebraic geometry.

Marie-France Vigneras, Université Paris-Diderot For contributions to the Langlands program and the theory of automorphic forms.

Luc Vinet, Université de Montréal

For contributions to mathematical physics and special functions, and for service to the mathematical community.

Weiqiang Wang, University of Virginia For contributions to Lie theory and representation theory and service to the mathematical community.

Richard M. Weiss, Tufts University For contributions to group theory and geometry, and for exposition.

Sarah Witherspoon, Texas A\&M University For contributions to representation theory and cohomology of Hopf algebras, quantum groups, and related objects, and for service to the profession and mentoring.

Milen Yakimov, Louisiana State University, Baton Rouge For contributions to noncommutative algebra and service to the mathematical community.

Catherine Huafei Yan, Texas A\&M University

For contributions to combinatorics and discrete geometry.

Photo Credit

Photos by Kate Awtrey, Atlanta Convention Photography.

See more at: www.ams.org/profession/ams

-fe11ows/new-fe11ows. 


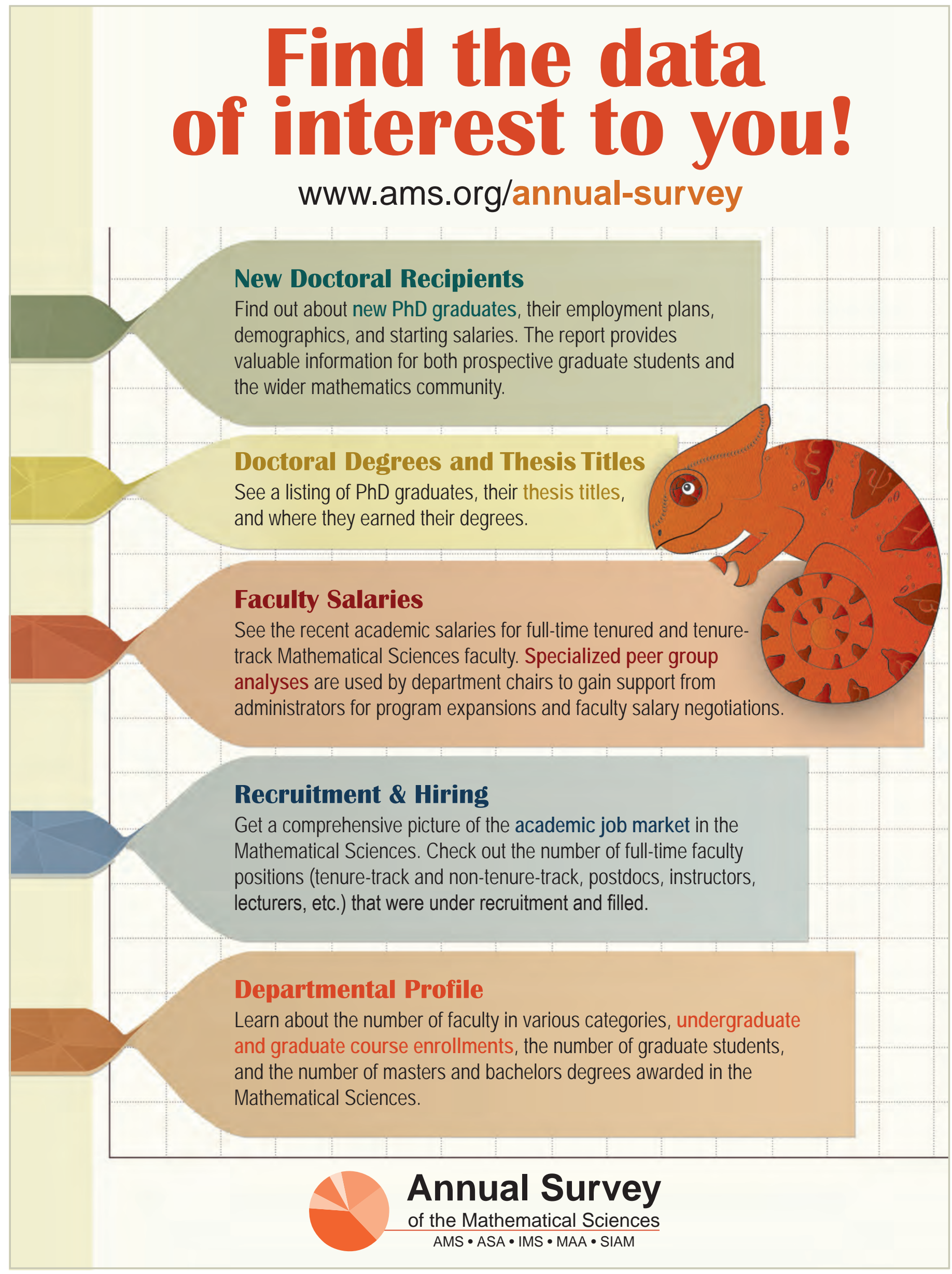




\section{ANNUAL SURVEY}

\section{Report on the 2015-2016 New Doctoral Recipients}

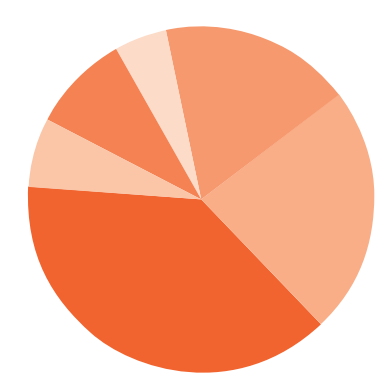

\section{Amanda L. Golbeck, Thomas H. Barr, and Colleen A. Rose}

This report presents a statistical profile of recipients of doctoral degrees awarded by departments in the mathematical sciences at universities in the United States during the period July 1, 2015 through June 30, 2016. Information in this report was provided by 298 of the 322 doctoral-granting departments surveyed, with additional information provided by the individual new doctoral recipients. Readers should keep in mind that the drops seen in the Statistics and Biostatistics groups are likely the result of nonresponse; 23 (10 Statistics \& 13 Biostatistics) of 105 departments did not respond in time for the publication of this report.

The 2015-2016 Report on Employment Experiences of New Doctoral Recipients immediately following this report provides an analysis of the fall 2016 employment plans of the $730 \mathrm{PhD}$ recipients who responded to this survey, as well as a summary of their demographic characteristics.

Detailed information, including tables not appearing in this report, is available on the AMS website at www.ams.org/ annual-survey.

\section{Doctoral Degrees Awarded}

In mathematical sciences $1,921 \mathrm{PhDs}$ were awarded by 279 doctoral-granting departments; 19 doctoral-granting departments awarded no doctoral degrees.

The highest percentage, 30\% (575) of the new PhDs had a dissertation in statistics/biostatistics, followed by algebra/number theory with 15\% (279) and applied mathematics with 13\% (248).

Comparing PhDs awarded in 2015-16 to 2014-15 the number of PhDs awarded:

- Increased about $1 \%$ from 1,901 to 1,921 . In the 272 departments that responded both this year and last year the number of PhDs awarded decreased from 1,901 to 1,871.

- Increased in all groups except Applied Math, Statistics, and Biostatistics.

- Increased 22\% in Math Public Small, 28\% in Math Private Small, $10 \%$ in Math Private large, and less than 3\% in both Math Public Large and Medium.

- Decreased 7\% in Applied Math, 16\% in Statistics, and 9\% in Biostatistics.
Figure A.1: Number and Percentage of Degrees Awarded by Department Grouping*

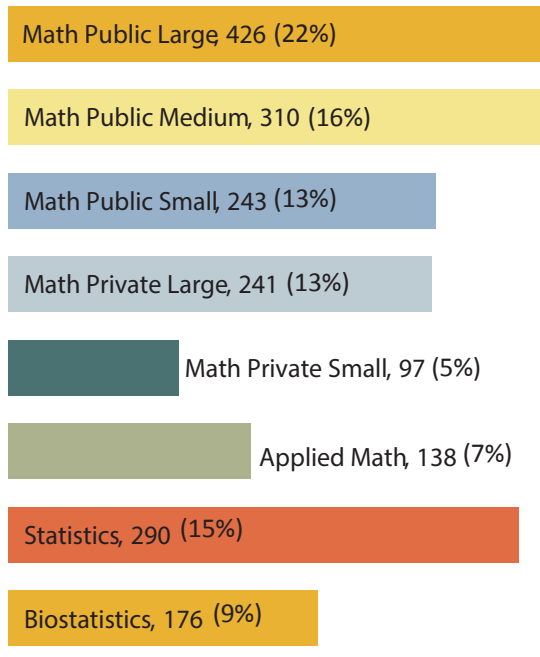

Total Degrees Awarded: 1,921

*See page 358 for a description of the department groupings. 


\section{ANNUAL SURVEY}

Figure A.2: New PhDs Awarded by Group

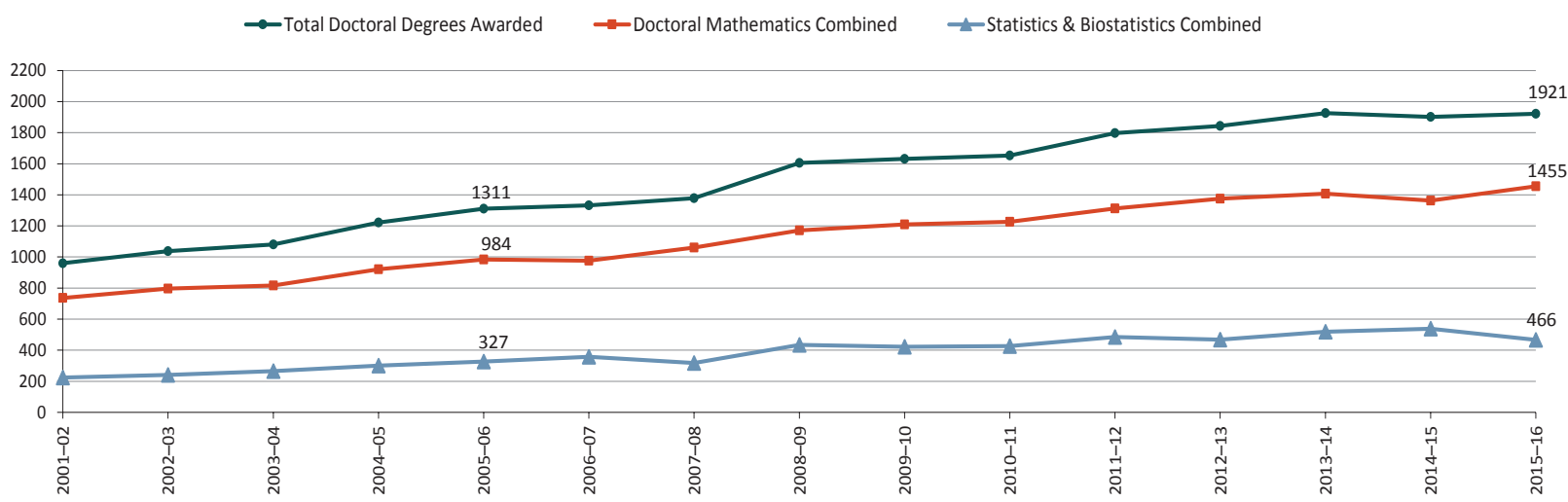

Comparing PhDs awarded in 2015-16 with those awarded in 2005-06:

- PhDs awarded have increased more than $46 \%$ over the last 10 years.

- Degrees awarded by Doctoral Math and by Statistics/Biostatistics combined have increased $48 \%$ and 43\%, respectively.

\section{Employment}

The employment plans are known for 1,746 of the 1,921 new doctoral recipients. The number of new doctoral recipients employed in the US is 1,449, essentially the same as last year. Among those new PhDs employed in Doctoral Math departments, $68 \%$ are in postdoc positions, down from $75 \%$ last year. The number of new PhDs taking positions in Business \& Industry is 495; last year's count was 492. All groups except Math Public Medium, Statistics and Biostatistics showed an increase in Business \& Industry. The overall US unemployment rate for the new doctoral recipients is $5.9 \%$, essentially the same as the $6.1 \%$ in 2014-15. (Details on the calculations are on page 357. )

Figure E.1: Employment Status

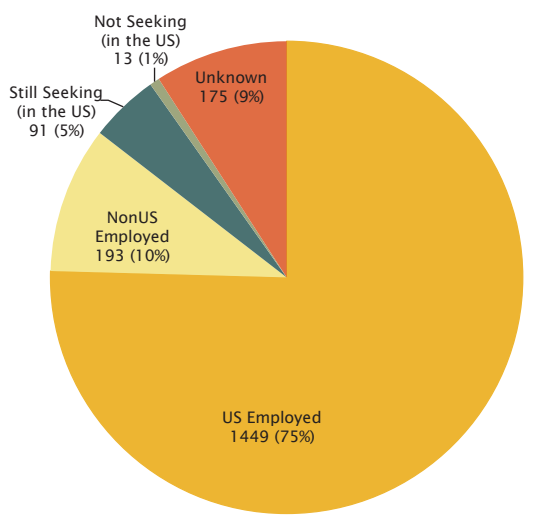

- $52 \%$ (757) of those who are employed in the US are US citizens, up from $50 \%$ in $2014-15$.

- $\quad 79 \%$ (692) of non-US citizens whose employment status is known are employed in the US, the remaining 179 non-US citizens are either employed outside of the US or are unemployed.

- $8 \%$ (133) of the new PhDs who are employed are working at the institution that granted their degree, down from 9\% last year. These individuals constitute $15 \%$ of total US academic employed.

- $57 \%$ of those still seeking employment in the US are US citizens.
Figure E.2: US Employed by Type of Employer

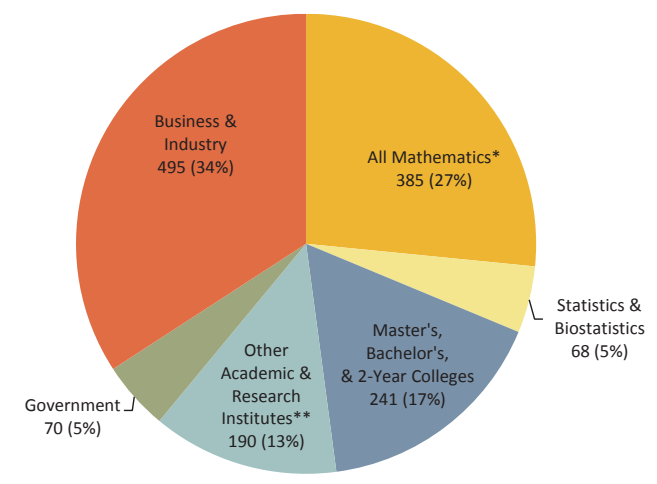

*Includes all Math Public, Math Private, and Applied Math departments. $* *$ Other Academic consists of departments outside the mathematical sciences including numerous medical-related units.

- $\quad$ US academic hiring increased 2\% to 884 compared to 864 last year.

- $\quad$ Government hiring decreased 20\% (from 88 to 70); all doctoral-granting groups except Math Public Medium, Math Private Large, Math Private Small, and Applied Math showed decreases in the number of PhDs taking positions in this sector. 


\section{ANNUAL SURVEY}

Figure E.3: Employment in the US by Type of Employer and Citizenship Total: 1,449

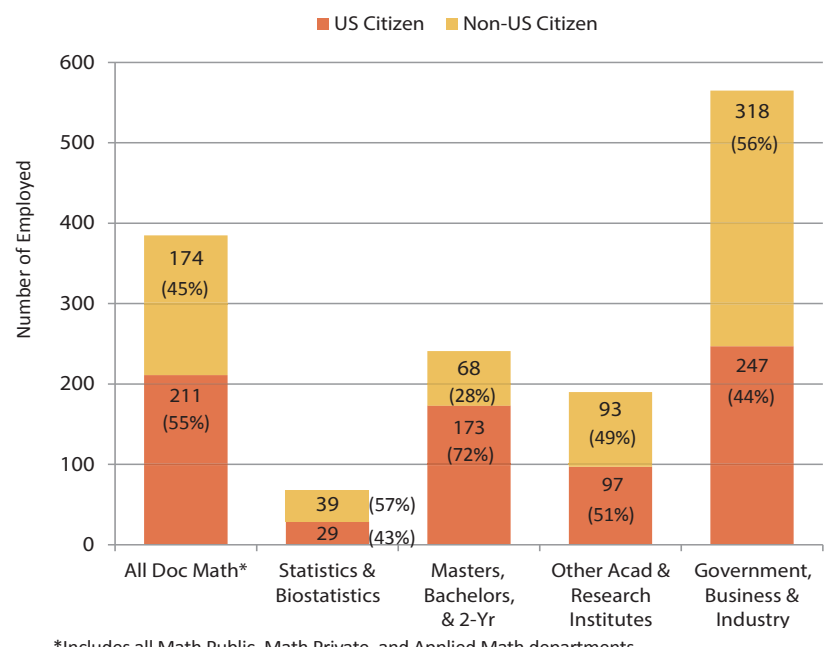

- Total known to be employed as of this report: 1,642

Of the US Citizens whose employment status is known, 87\% (757) are employed in the US, and of these:

- $\quad 32 \%$ are employed in PhD-granting departments.

- $\quad 36 \%$ are employed in all other academic categories.

- $\quad 33 \%$ are employed in government, business and industry.

- $35 \%$ (576) of the new PhDs that are employed are reported to be in postdoc positions, down $4 \%$ from 603 in 2014-15.

- $\quad 52 \%$ of the new PhDs awarded by the Math Private Large group are employed in postdocs, while only $16 \%$ of new PhDs awarded by the Math Public Small group and $17 \%$ of $\mathrm{PhDs}$ awarded by the Statistics group are in postdocs.

Figure E.4: PhDs Employed in Postdocs by Degree-Granting Department Group

Number of PhDs in Postdocs

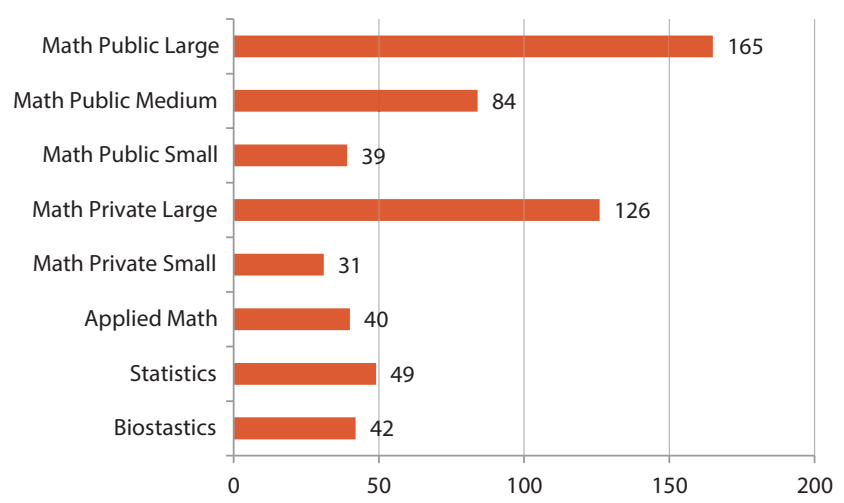

- $\quad 48 \%$ of the new PhDs having US academic employment are in postdocs, down from $51 \%$ last year.

Looking at Figure E.5, we see that:

- US citizens hold the majority of postdoc positions in the employment sectors of Masters, Bachelors, \& 2-Year, and Government and Business/Industry. In other sectors they hold between $41-49 \%$ of postdoc positions.

- $\quad 22 \%$ of the new PhDs in postdoc positions are employed outside the US; the same percentage as 2014-15.

- $\quad 71 \%$ of the new PhDs employed in Doctoral Math departments are in postdoc positions, down from $75 \%$ last year.

Figure E.5: New PhDs Employment by Citizenship, Type of Position, and Type of Employer

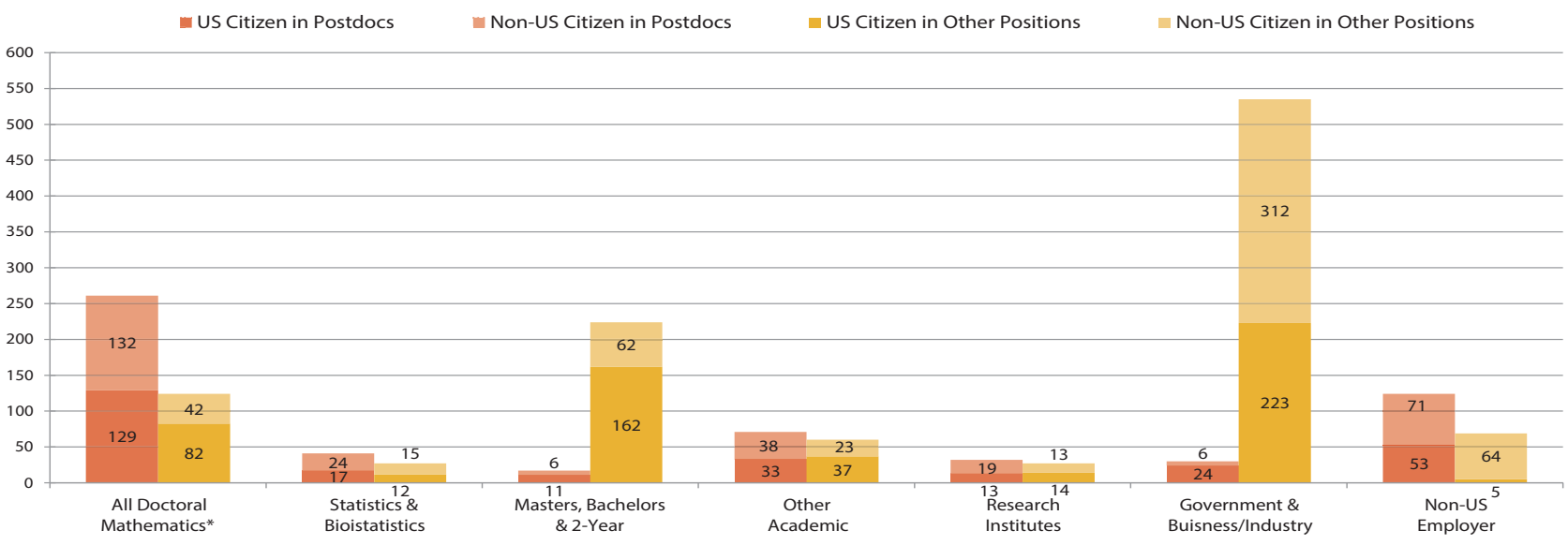

*Includes all Math Public, Math Private, and Applied Math departments. 


\section{ANNUAL SURVEY}

Figure E.6 displays the US unemployment rate for new doctorates; details on the calculations are on page 781 .

Figure E.6: Percentage of New Doctoral Recipients Unemployed 2007-16*

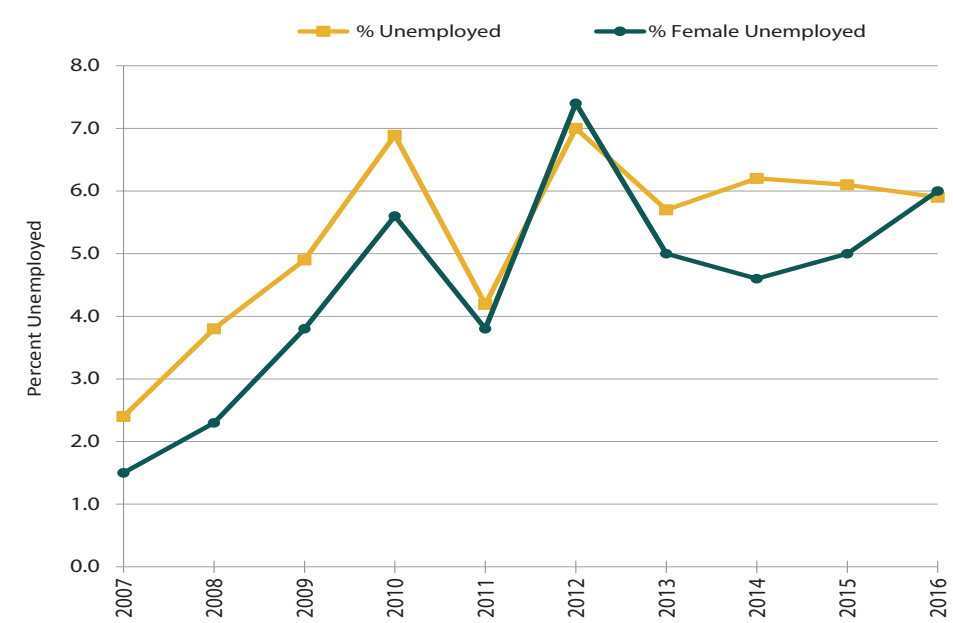

Among new doctorates reported to be in the US and whose employment status is known:

- Overall unemployment is $5.9 \%$.

- $6.4 \%$ of US citizens are unemployed, compared to $6.7 \%$ in fall 2015.

- $5.3 \%$ of non-US citizens are unemployed, essentially the same as the 2015 rate.

- $\quad$ New doctorates from the Math Private Small Group reported the highest unemployment rate at $13.7 \%$, essentially unchanged from $13.1 \%$ last year.

- $\quad$ New doctorates from the Biostatistics Group reported the lowest unemployment at 2.3\%.

Figure E.7: Percentage of Employed New PhDs by Type of Employer

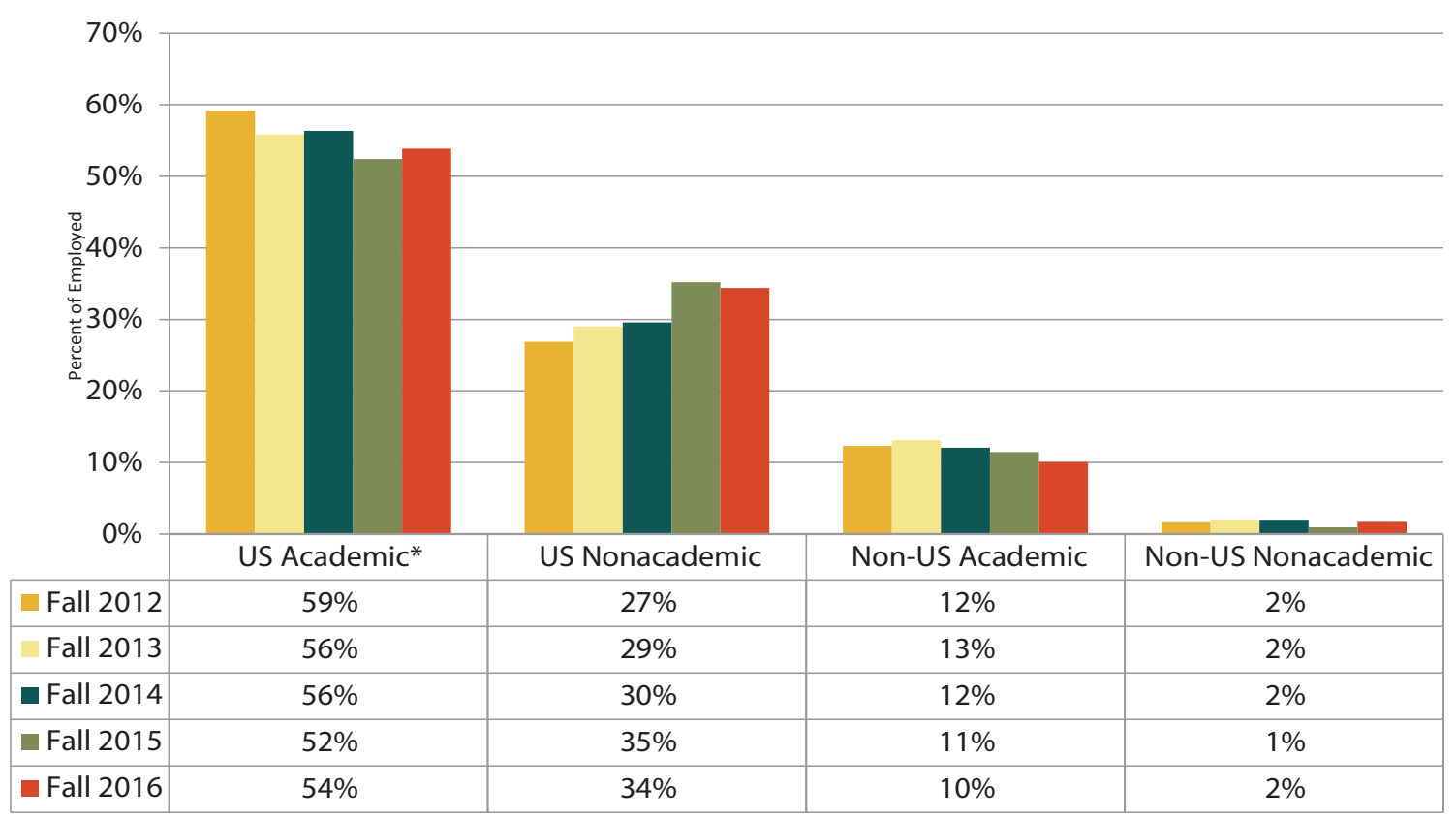

* Includes other academic departments and research institutes/other non-profits.

Here are a few notable features of Figure E.7 for 2016.

- US academic hiring increased to 54\%, while US nonacademic hiring dropped to 34\%.

- Non-US academic hiring dropped to $10 \%$ (a five-year low).

- Detailed information on new PhDs employed in the US by degree-granting department group is available on the AMS website at www. ams.org/annua1-survey. 


\section{ANNUAL SURVEY}

\section{Demographics}

Gender and citizenship were known for all 1,921 new PhDs reported for 2015-16. The percentage of US citizens is $49 \%$, up from $46 \%$ last year. Females accounted for $27 \%$ of the US citizen total, down from $31 \%$ last year. Non-US citizens receiving a $\mathrm{PhD}$ decreased to $51 \%$ from $54 \%$ last year. 10\% (66) of the non-US citizens employed in the US have permanent visa status, almost unchanged from 9\% last year. 5\% (95) of PhDs awarded were to underrepresented minorities.

Figure D.1: Gender of Doctoral Recipients by Degree-Granting Grouping

Male Female Neither

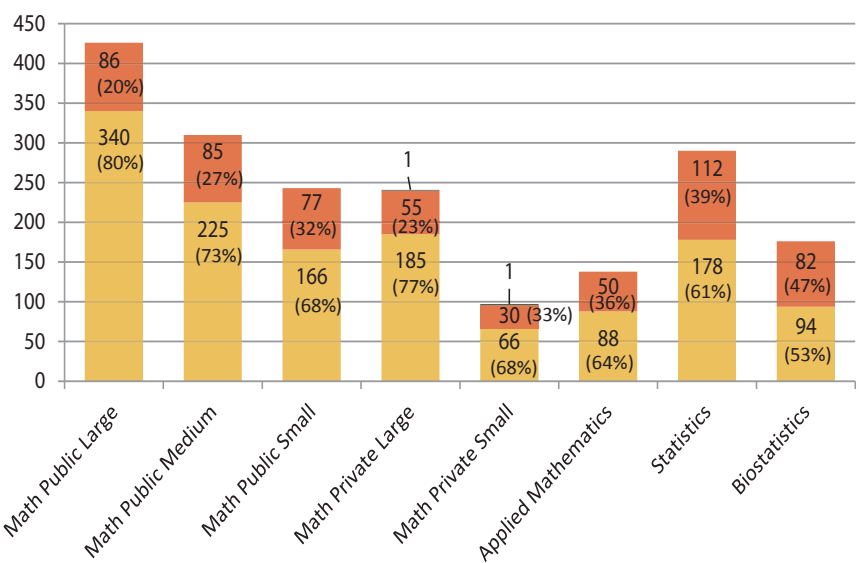

- Females account for 30\% (576) of 1,921 PhDs, down from $31 \%$ last year.

- Two individuals gender-identified as neither male or female.

Figure D.3: Gender of US Citizen Doctoral Recipients by Degree-Granting Grouping

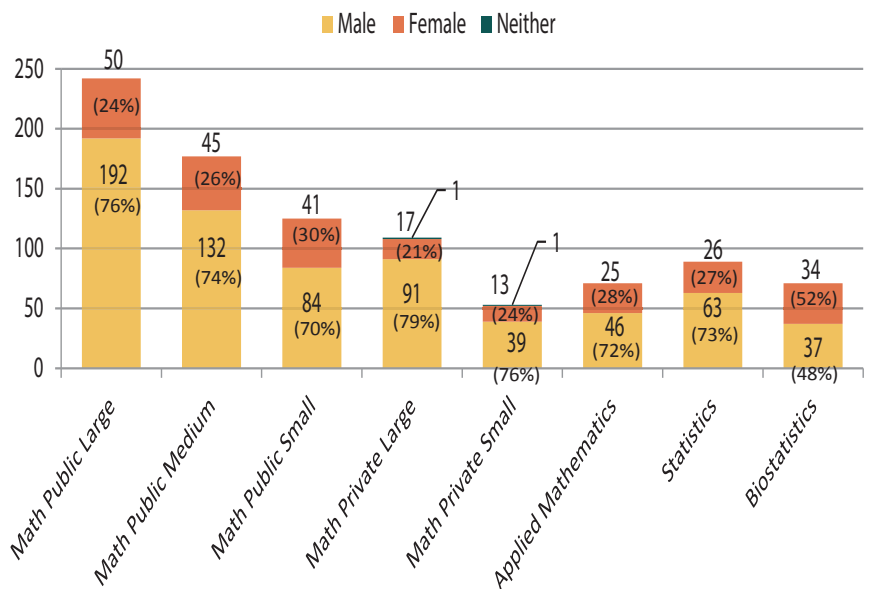

- $51 \%$ of the males, $44 \%$ of the females, and $100 \%$ of the neither are US citizens.

- $\quad$ Females accounted for $27 \%$ of the US citizens.

- Among the US citizens: 2 are American Indian or Alaska Native, 75 are Asian, 29 are Black or African American, 45 are Hispanic or Latino, 7 are Native Hawaiian or Other Pacific Islander, 754 are White, and 25 are of unknown race/ethnicity.

- Math Public Large awarded the highest number (23) of PhDs to US citizen minorities, while Biostatistics awarded the smallest number (2), followed by Statistics with 3.
Figure D.2: Citizenship of Doctoral Recipients by Degree-Granting Grouping

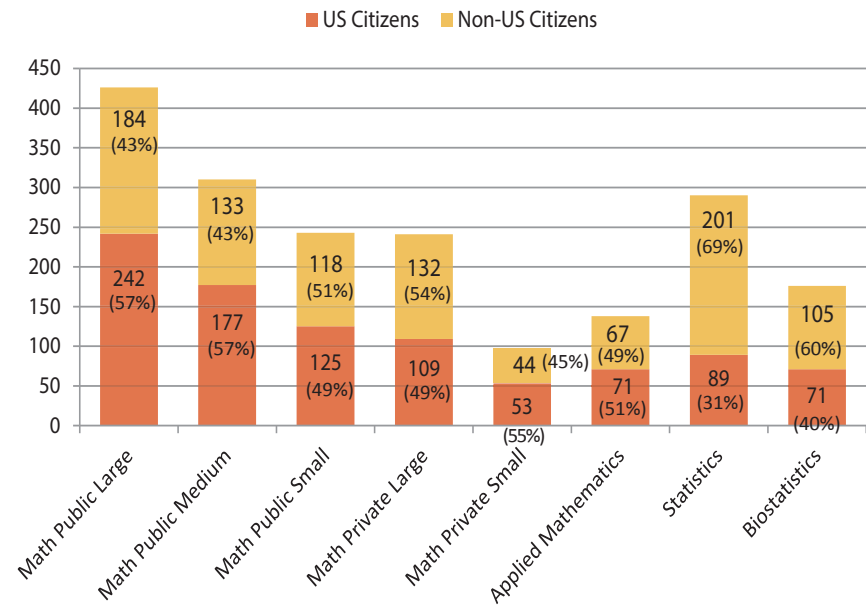

- $\quad 57 \%$ of all the PhDs awarded by the Math Public Large and Medium groups were to US citizens, while only $31 \%$ of the PhDs awarded by the Statistics group were to US citizens.

- $\quad$ All groups except Math Private Large, Statistics, and Biostatistics awarded more PhDs to US citizens than Non-US citizens.

Figure D.4: Citizenship of New PhD Recipients, 2010-16

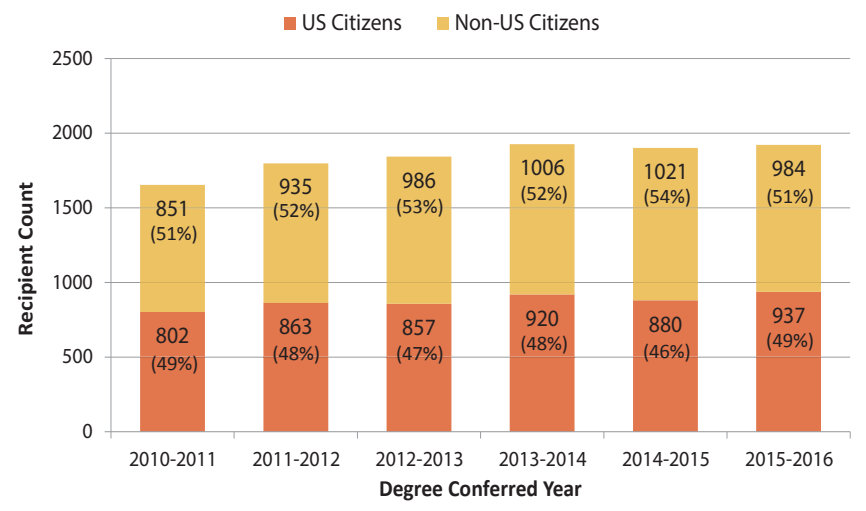

Looking at the last six years, we see that:

- The proportion of PhDs awarded to US citizens is at a six-year high, $49 \%$ (937). While this is a $7 \%$ increase from last year, it is the same percentage as in fall 2010-11.

- $\quad$ Non-US citizen counts decreased 4\% to 984 from 1,021 last year. While this is the first year-to-year drop in six-years the non-US citizen count has increased $16 \%$ over that in 2010-11. 


\section{Female New Doctoral Recipients}

The proportion of female new doctoral recipients is 30\% essentially unchanged from 31\% last year. Of the 1,049 new PhDs hired into academic positions, 31\% (320) were women, compared with 30\% as last year. Twenty-six percent of those hired into postdoc positions were women, with $42 \%$ of the women in postdocs being US citizens, down from $45 \%$ last year. The US unemployment rate for females is $6.0 \%$, compared to $5.9 \%$ for males and $5.9 \%$ overall.

Figure F.1: Females as a Percentage of New Doctoral Recipients Produced by and Hired by Department Grouping

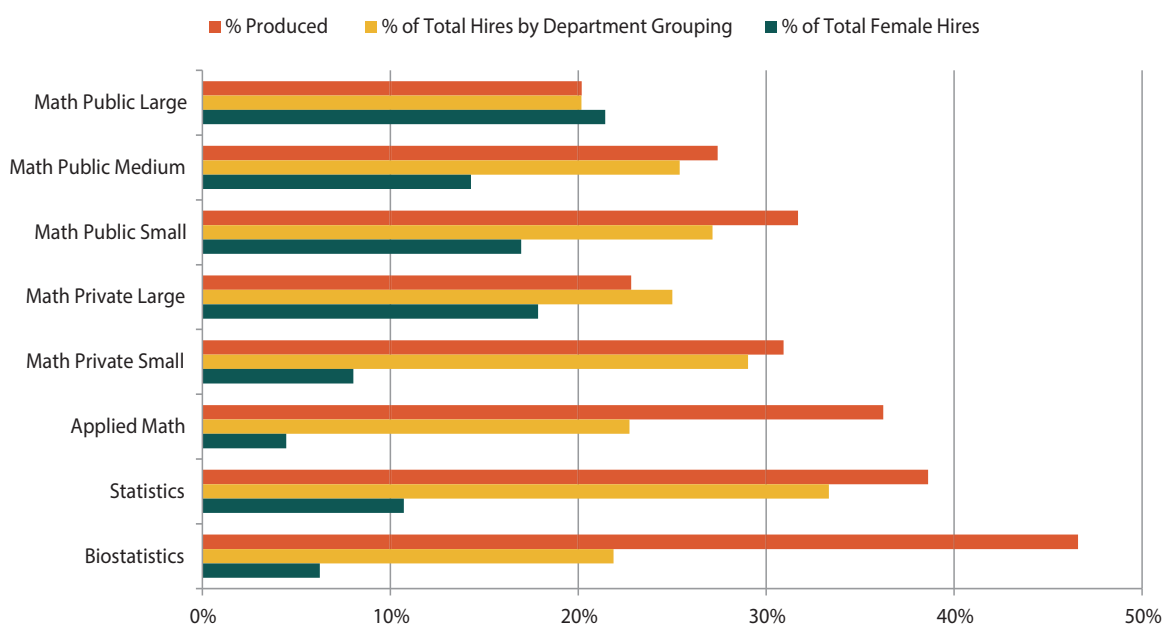

Table F.1: Number of Female New Doctoral Recipients Produced by and Hired by Department Groupings

\begin{tabular}{|l|c|c|}
\hline \multicolumn{1}{|c|}{$\begin{array}{c}\text { Department } \\
\text { Grouping }\end{array}$} & $\begin{array}{c}\text { Females } \\
\text { Produced }\end{array}$ & $\begin{array}{c}\text { Females } \\
\text { Hired }\end{array}$ \\
\hline Math Public Large & 86 & 24 \\
\hline Math Public Medium & 85 & 16 \\
\hline Math Public Small & 77 & 19 \\
\hline Math Private Large & 55 & 20 \\
\hline Math Private Small & 30 & 9 \\
\hline Applied Math & 50 & 5 \\
\hline Statistics & 111 & 12 \\
\hline Biostatistics & 82 & 7 \\
\hline Total & $\mathbf{5 7 6}$ & $\mathbf{1 1 2}$ \\
\hline
\end{tabular}

- $32 \%$ of those hired into US academic positions were female (up from 31\% last year).

- $42 \%$ of those hired by the Bachelor's Group were women (same as last year), $38 \%$ of those hired by the Master's Group were women (up from 26\% last year), and 24\% of those hired by the PhD Math groups combined were women.

- $41 \%$ of those hired into Research Institutes/Other non-profit positions were women (up from 26\% last year).

- $33 \%$ of those hired into Government positions were women (down from $42 \%$ last year).

- $60 \%$ of the women employed in all doctoral groups are in postdoc positions, compared to $69 \%$ of males employed in these groups.

Figure F.2: Females as a Percentage of US Citizen Doctoral Recipients and Graduate Students

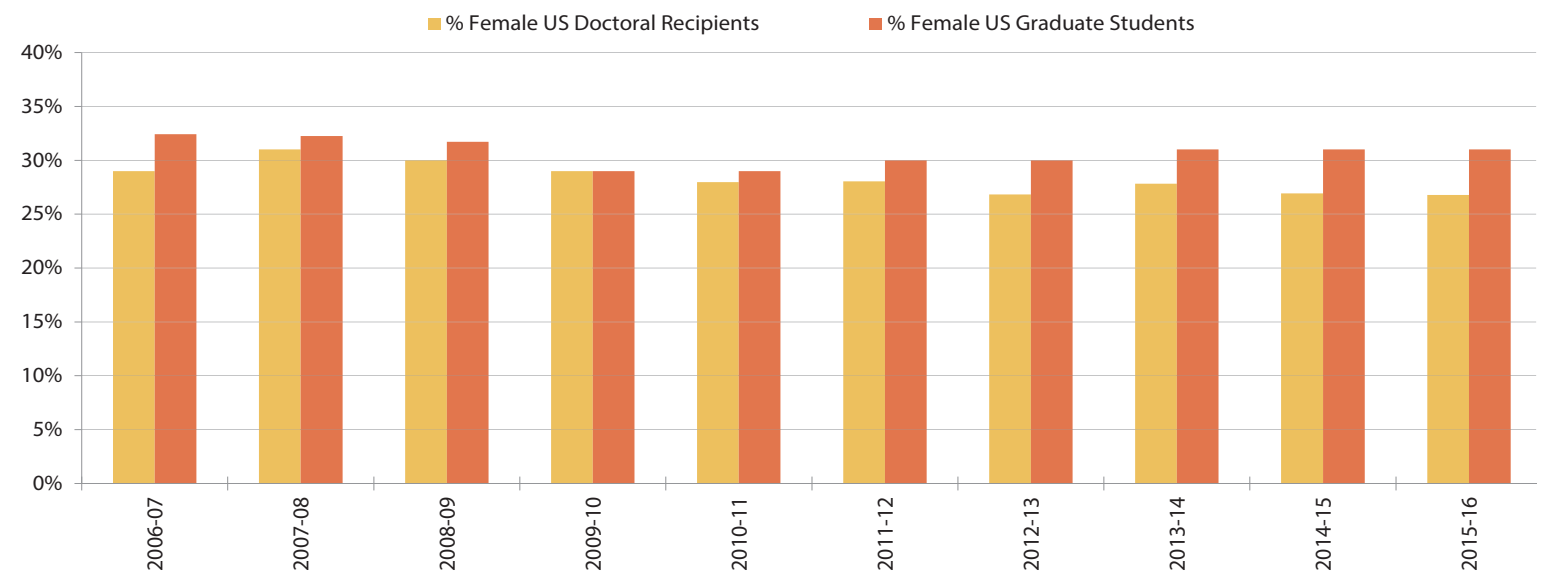




\section{ANNUAL SURVEY}

\section{PhDs Awarded by Statistics and Biostatistics (Stat/Biostat) Departments}

This section contains information about new doctoral recipients as reported by 82 (49 of 59 statistics and 33 of 46 biostatistics) Stat/Biostat departments that responded to this survey. These departments produced 466 new doctorates, all of whom had dissertations in statistics/biostatistics. This is a 13\% decrease in the number reported for fall 2015, which was 538. In addition, Math Public, Math Private and Applied Math departments combined had $110 \mathrm{PhD}$ recipients with dissertations in statistics. 34\% (160) of the new PhDs awarded by Stat/Biostat are US citizens (while in the other groups combined, 53\% are US citizens). The unemployment rate for US citizens with new Stat/Biostat PhDs is $3.0 \%$, essentially the same as in 2014-15. Underrepresented minorities received 6\% of all degrees awarded to US citizens and permanent residents in Stat/Biostat (199).

Figure S.1: PhDs Awarded by Statistics/Biostatistics Departments

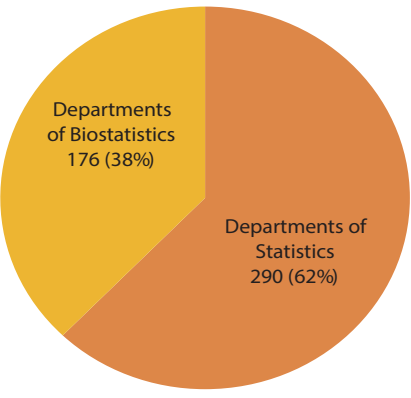

- $\quad 24 \%$ of all mathematical sciences PhDs awarded were in the Stat/ Biostat group.

- Females account for $38 \%$ of Statistics and $47 \%$ of Biostatistics PhDs awarded.

Figure S.4: Employment Status of PhD Recipients from

Statistics/Biostatistics Departments

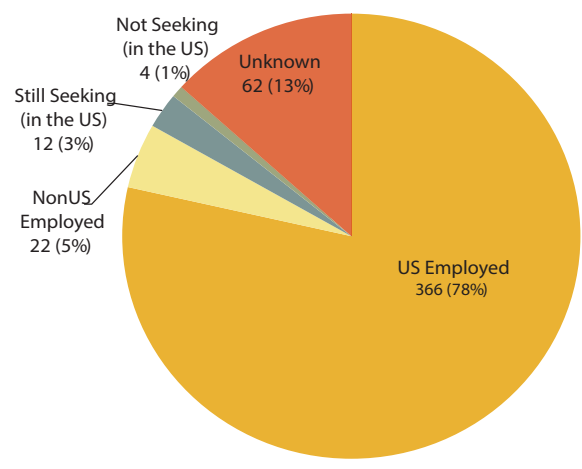

Total PhDs Awarded: 466

- 3.2\% of Stat/Biostat PhDs are unemployed, (compared to 6.8\% among Doctoral Math), essentially unchanged from 2014-15.

- Unemployment among new PhDs with dissertations in statistics/biostatistics is $2.6 \%$, down from 3.8\%. Among all other dissertation groupings, 5.6\% are unemployed.
Figure S.2: Gender of PhD Recipients from Statistics/Biostatistics Departments

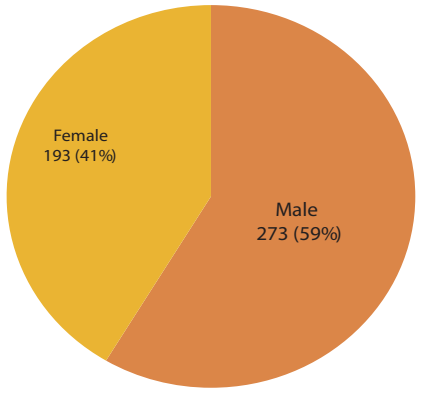

emales accounted for $41 \%$ of the $466 \mathrm{PhDs}$ in Stat/Biostat, compared to Doctoral Math, where $26 \%$ are female.
Figure S.3: Citizenship of PhD Recipients from Statistics/Biostatistics Departments

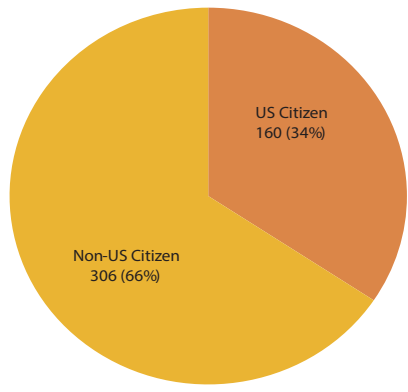

$38 \%$ of Stat/Biostat US citizen $\mathrm{PhD}$ recipients are females, while in Doctoral Math 25\% of the US citizens are females.
Figure S.5: US-Employed PhD Recipients from Statistics/Biostatistics Departments by Type of Employer

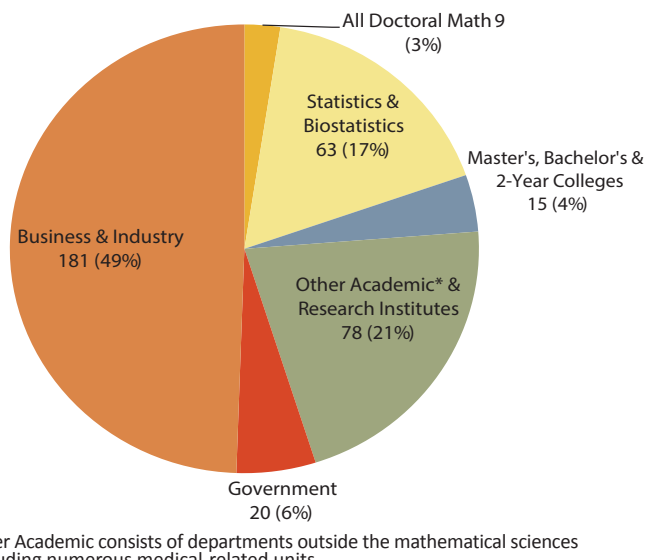

Total US Employed: 366

- $\quad 49 \%$ of Stat/Biostat PhDs are employed in Business/Industry, compared to 29\% in Math.

- $28 \%$ of those hired by Stat/Biostat were females, compared to $24 \%$ in Math. 


\section{Remarks on US Unemployment Rate Calculations}

In the unemployment calculations provided in this report, the individuals employed outside the US have been removed from the denominator used in the calculation of the rate, in addition to the routine removal of all individuals whose employment status is unknown. This is a change from Annual Survey Reports prior to 2009. As a consequence, the unemployment rate now being reported more accurately reflects the US labor market experienced by the new doctoral recipients. This change tends to increase the rate of unemployment over that reported in prior years.
Another small change from prior years is that, those individuals reported as not seeking employment have also been removed from the denominator. The number of individuals so designated is small each year, and the impact of this change is to produce a slight increase in the rate over that reported in prior years.

The unemployment rates for years prior to 2009 shown in this report have been recalculated using this method. One can view a comparison of the unemployment rates using the earlier method and the currentmethod by visiting the AMS website at www.ams.org/annual-survey/ surveyreports.html.

\section{Departmental Groupings and Response Rates}

Starting with reports on the 2012 AMS-ASA-IMS-MAASIAM Annual Survey of the Mathematical Sciences, the Joint Data Committee has implemented a new method for grouping the doctorate-granting mathematics departments. These departments are first grouped into those at public institutions and those at private institutions. These groups are further subdivided based on the size of their doctoral program as reflected in the average annual number of PhDs awarded between 2000 and 2010, based on their reports to the Annual Survey during this period. Furthermore, doctorate-granting departments which self-classify their $\mathrm{PhD}$ program as being in applied mathematics will join with the other applied mathematics departments previously in Group Va to form their own group. The former Group IV will be divided into two groups, one for departments in statistics and one for departments in biostatistics.

For further details on the change in the doctoral department groupings, see the article in the October 2012 issue of Notices of the AMS at www.ams.org/ notices/201209/rtx120901262p.pdf.

\section{Department Grouping Response Rates}

\section{Doctorates Granted \\ Departmental Response Rates by Grouping}

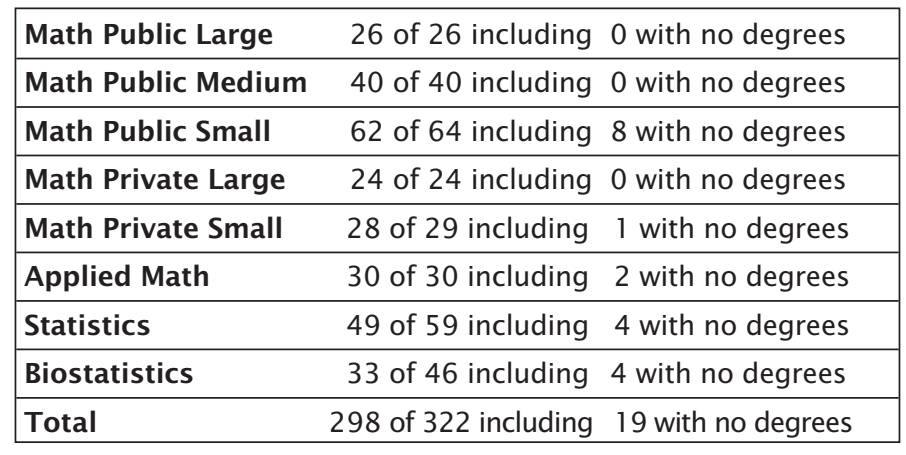

As of press time for this issue of Notices, the following departments had not responded to the survey. Therefore, any PhDs which may have been awarded by these departments are not included in this report.

\section{Mathematics Departments}

Yeshiva University
Statistics Departments

George Washington University

Michigan State University

North Carolina State University

Oklahoma State University

University of Arizona

University of California, Davis

University of California, Los Angeles

University of California, Santa Barbara

University of Virginia

Western Michigan University

\section{Biostatistics Departments}

Brown University

Columbia Univesity

Georgia Southern University, College of Public Health

Saint Louis University College for Public Health \& Social Justice

University of Cincinnati, Medical College

University of Colorado, Denver

University of Illinois at Chicago

University of Kentucky

University of Louisville

University of Oklahoma, Health Science Center

University of South Carolina

University of South Florida

Virginia Commonwealth University, Medical Center 


\section{ANNUAL SURVEY}

\section{Department Groupings}

In this report, Mathematical Sciences departments are those in four-year institutions in the US that refer to themselves with a name that incorporates (with a few exceptions) "Mathematics" or "Statistics" in some form. For instance, the term includes, but is not limited to, departments of "Mathematics," "Mathematical Sciences," "Mathematics and Statistics," "Mathematics and Computer Science," "Applied Mathematics," "Statistics," and "Biostatistics." Also, Mathematics (Math) refers to departments that (with exceptions) have "mathematics" in the name; Stat/Biostat refers to departments that incorporate (again, with exceptions) "statistics" or "biostatistics" in the name but do not use "mathematics." The streamlining of language here militates against the possible objection to foreshortening the full subject names.
Starting with reports on the 2012 AMS-ASA-IMS-MAASIAM Annual Survey of the Mathematical Sciences, the Joint Data Committee implemented a new method for grouping doctorate-granting Mathematics departments. These departments are first grouped into those at public institutions and those at private institutions. These groups are further subdivided based on the size of their doctoral program as reflected in the average annual number of PhDs awarded between 2000 and 2010, based on their reports to the Annual Survey during that period.

For further details on the change in the doctoral department groupings, see the article in the October 2012 issue of Notices of the AMS at www.ams.org/journals/ notices/201209/rtx120901262p.pdf

\footnotetext{
Math Public Large consists of departments with the highest annual rate of production of PhDs, ranging between 7.0 and 24.2 per year. Math Public Medium consists of departments with an annual rate of production of PhDs, ranging between 3.9 and 6.9 per year. Math Public Small consists of departments with an annual rate of production of PhDs of 3.8 or less per year.

Math Private Large consists of departments with an annual rate of production of PhDs, ranging between 3.9 and 19.8 per year.

Math Private Small consists of departments with an annual rate of production of PhDs of 3.8 or less per year.

Applied Mathematics consists of doctoral-degree-granting applied mathematics departments.

Statistics consists of doctoral-degree-granting statistics departments.

Biostatistics consists of doctoral-degree-granting biostatistics departments.

Masters contains US departments granting a master's degree as the highest graduate degree.

Bachelors contains US departments granting a baccalaureate degree only.

Doctoral Math contains all US math public, math private, and applied math mathematics departments granting a PhD as the highest graduate degree.

Mathematics (Math) contains all US Math Public, Math Private, and Applied Math, Masters, and Bachelors Groups above.

Stat/Biostat contains all doctoral-degree-granting statistics and biostatistics departments.
}

Listings of the actual departments that compose these groups are available on the AMS website atwww. ams . org/ annua1-survey/groups.

\section{Doctoral Degrees Conferred 2015-2016}

\section{Supplementary List}

The following list supplements the list of thesis titles published in the February 2017 Notices, pages 281-301.

\section{TEXAS}

\section{Southern Methodist University (1)}

\section{Statistical ScIENCES}

Chang, Po-Yao, Self-shrinkers to the mean curvature flow asymptotic to isoparametric cones.

\section{Ohio}

\section{University of Toledo (5)}

\section{MATHEMATICS AND STATISTICS}

Karki, Manoj, Invariant Riemannian metrics in four dimensional Lie groups.

Liu, Gang, A new approach to ANOVA methods for autocorrelated data.

Mei, Jingning, Inference for autoregressive coefficients and error distribution.

Pokharel, Krishna, An isospectral flow for complex upper Hessenberg matrices.

Tang, Lin, Efficient inference for periodic autoregressive coefficients with polynomial spline smooth approach. 


\section{PENNSYLVANIA}

Bryn Mawr College (1)

MATHEMATiCs

Bryant, Kathryn,Sliceimplies mutant ribbonfor odd, stranded pretzel knots.

\section{VIRGINIA}

\section{George Mason University (2)}

MATHEMATICAL SCIENCES

Locke, Rachel, Multiplication operators in discrete settings of an infinite graph and the discrete Zygmund space.

Stephens, Thomas, Topological methods for evolution equations.

\section{TEXAS}

\section{Southern Methodist University (1)}

STATISTICAL SCIENCES

Liao, Yijie, Marginal posterior distribution of regression parameters for the Cox model under Dirichlet and gamma process priors.

\section{Acknowledgments}

The Annual Survey attempts to provide an accurate appraisal and analysis of various aspects of the academic mathematical sciences scene for the use and benefit of the community and for filling the information needs of the professional organizations. Every year, college and university departments in the United States are invited to respond. The Annual Survey relies heavily on the conscientious efforts of the dedicated staff members of these departments for the quality of its information. On behalf of the Data Committee and the Annual Survey Staff, we thank the many secretarial and administrative staff members in the mathematical sciences departments for their cooperation and assistance in responding to the survey questionnaires.

The Annual Survey is co-sponsored by the American Mathematical Society (AMS), the American Statistical Association (ASA), Institute for Mathematical Statistics (IMS), Mathematical Association of America (MAA), and the Society for Industrial and Applied Mathematics (SIAM). 


\section{ANNUAL SURVEY}

\section{Report on the 2015-2016 Employment Experiences of the New Doctoral Recipients}

\section{Amanda L. Golbeck, Thomas H. Barr, and Colleen A. Rose}

This report provides information on employment gathered from a subset of the 2015-16 new PhDs on the Employment Experiences of New Doctoral Recipients (EENDR) Survey. It expands on the details of employment that are not available through the departments.

The EENDR survey was sent to the 1,656 new PhDs for which departments provided current contact information by early October of 2016. Of these individuals, 730 (44\%) responded. The employment status is known for 721 of these individuals. Of the 698 who reported being employed, $4 \%$ are part-time and $24 \%$ indicated they were actively looking for new employment. The US unemployment among this group is $2.8 \%$

\section{Figure EE.1: EENDR Respondents Reporting Permanent US Employment by Sector}

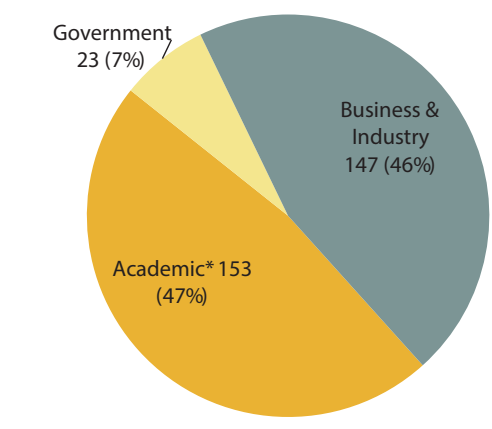

* Includes research institutes and other non-profits.

Of the 323 permanently employed:

- $37 \%$ are women.

- $63 \%$ of those reporting academic employment hold tenured/ tenure-track positions (up from $58 \%$ last year).
Figure EE.2: EENDR Respondents Reporting Temporary US Employment by Sector

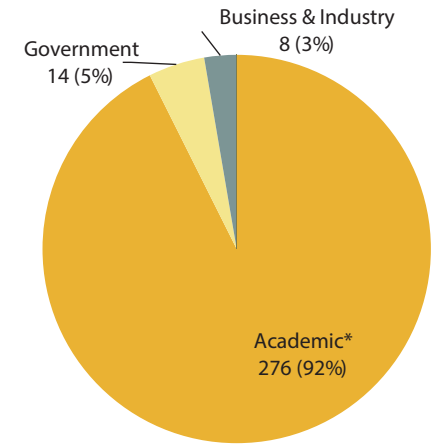

* Includes research institutes and other non-profits.

Of the 298 temporarily employed:

- $\quad 31 \%$ are women.

- $\quad 46 \%$ were unable to find a suitable permanent position (down from $47 \%$ last year).

- $\quad 72 \%$ are employed in postdocs, and $38 \%$ of these reported they could not find a suitable permanent position.
Figure EE.3. EENDR Respondents Employed Outside the US by Sector

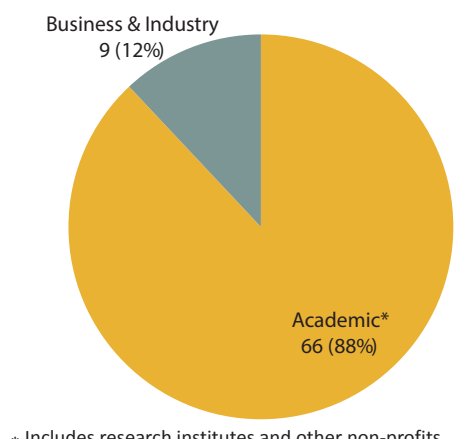

Of the 75 employed outside the US:

- $17 \%$ are women.

- $\quad 17 \%$ are US citizens.

- $\quad 72 \%$ are employed in postdocs. 
Table EE.1: Number and Percentage of EENDR Respondents Employed in the US by Job Status

\begin{tabular}{|c|c|c|c|c|c|c|c|c|c|c|c|}
\hline \multirow[b]{2}{*}{ Year } & \multirow[b]{2}{*}{$\begin{array}{l}\text { Perm } \\
\text { Total }\end{array}$} & \multirow[b]{2}{*}{$\%$} & \multirow[b]{2}{*}{$\begin{array}{r}\text { Temp } \\
\text { Total }\end{array}$} & \multirow[b]{2}{*}{$\%$} & \multicolumn{2}{|c|}{ Temporary } & \multicolumn{4}{|c|}{ Temporary Postdocs } & \multirow[b]{2}{*}{$\begin{array}{c}\#(\%) \\
\text { Unknown }\end{array}$} \\
\hline & & & & & $\begin{array}{c}\text { Perm } \\
\text { Not Avail }\end{array}$ & $\begin{array}{c}\% \text { of Temp } \\
\text { Total }\end{array}$ & Total & $\begin{array}{c}\% \text { of Temp } \\
\text { Total }\end{array}$ & $\begin{array}{c}\text { Perm } \\
\text { Not Avail }\end{array}$ & $\begin{array}{c}\% \text { of Temp } \\
\text { Postdocs }\end{array}$ & \\
\hline Fall 2012 & 261 & $44 \%$ & 328 & $56 \%$ & 127 & $39 \%$ & 242 & $74 \%$ & 108 & $45 \%$ & 0 \\
\hline Fall 2013 & 374 & $53 \%$ & 335 & $47 \%$ & 173 & $52 \%$ & 247 & $74 \%$ & 106 & $43 \%$ & 0 \\
\hline Fall 2014 & 363 & $51 \%$ & 343 & $49 \%$ & 148 & $43 \%$ & 260 & $76 \%$ & 88 & $34 \%$ & 0 \\
\hline Fall 2015 & 357 & $51 \%$ & 341 & $49 \%$ & 160 & $47 \%$ & 258 & $76 \%$ & 102 & $40 \%$ & 0 \\
\hline Fall 2016 & 323 & $52 \%$ & 298 & $48 \%$ & 136 & $46 \%$ & 214 & $72 \%$ & 82 & $38 \%$ & $2(<1 \%)$ \\
\hline
\end{tabular}

Table EE.1 compares the status of EENDR respondents employed in the US over the last five years:

- $52 \%$ of those employed for fall 2016 are in permanent positions. While this is higher than the proportion reported for fall 2015, it is lower than the high of 53\% for fall 2013.

- The proportion of those in temporary positions is $48 \%$, this is a drop from last year's figure and 8 percentage points lower than the five-year high of $56 \%$.

- $\quad 46 \%$ of those holding temporary positions were unable to find suitable permanent positions. While this is down from last year, it is higher than the five-year low of 39\% for fall 2012.

- $38 \%$ of those holding postdoc positions were unable to find suitable permanent positions. This figure is down seven percentage points from the five-year high of 45\%; and up four percentage points from the low in Fall 2014.

Table EE.2: Percentage of EENDR Respondents Employed in the US by Employment Sector within Job Status

\begin{tabular}{|c|c|c|c|c|c|c|}
\hline Year & \multicolumn{3}{|c|}{ Permanent } & \multicolumn{3}{c|}{ Temporary } \\
\cline { 2 - 7 } & Acad & Govn & B/I & Acad & Govn & B/I \\
\hline Fall 2012 & $61 \%$ & $8 \%$ & $32 \%$ & $92 \%$ & $5 \%$ & $2 \%$ \\
Fall 2013 & $53 \%$ & $7 \%$ & $40 \%$ & $92 \%$ & $4 \%$ & $4 \%$ \\
Fall 2014 & $54 \%$ & $6 \%$ & $40 \%$ & $92 \%$ & $5 \%$ & $3 \%$ \\
Fall 2015 & $44 \%$ & $8 \%$ & $48 \%$ & $93 \%$ & $3 \%$ & $4 \%$ \\
Fall 2016 & $47 \%$ & $7 \%$ & $46 \%$ & $93 \%$ & $5 \%$ & $3 \%$ \\
\hline
\end{tabular}

Looking at Table EE.2, we see that

- $\quad$ Permanent employment in the academic sector rebounded slightly to $47 \%$ after dropping to a fiveyear low of $44 \%$ last year, whereas employment in business/industry and goverment dropped to $46 \%$ and $7 \%$, respectively.

- Temporary employment in all three sectors has remained essentially unchanged over this fiveyear period.
Figure EE.4. Employment by Type of Educational Institution (Educ)

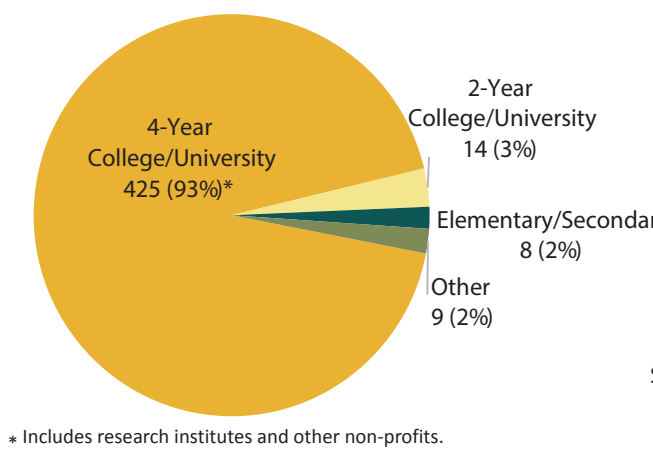

Looking at those employed in education

(456) we see that:

- US citizens hold 65\% of these positions; $62 \%$ are employed at a 4 -Year college/ university.

- Females hold 33\% of these positions; 63\% of these are US citizens and 30\% hold temporary positions.

- $66 \%$ of these positions are temporary; of those in temporary positions $62 \%$ are US citizens and $46 \%$ could not find a suitable permanent position.

- $31 \%$ of those employed in education are currently looking for another position.
Figure EE.5. Employment by Type of Government (Gov)

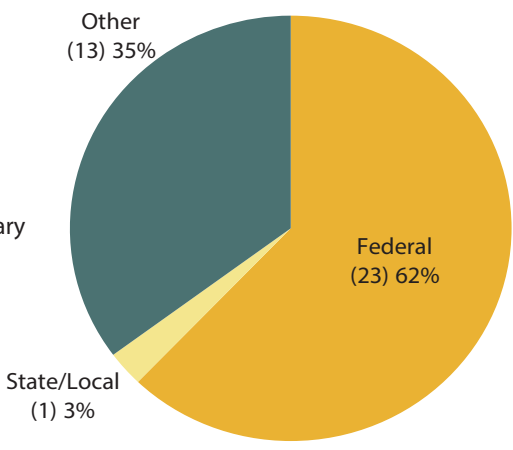

Looking at those employed in government (37) we see that:

- US citizens hold $47 \%$ of these positions.

- $38 \%$ are female; of these women, $64 \%$ work in the federal goverment.

- 38\% hold temporary positions; 86\% are US citizens and 8\% could not find a suitable permanent position.

- $87 \%$ of those employed in Gov are currently looking for another position.
Figure EE.6. Employment by Type of Business/Industry (BI)

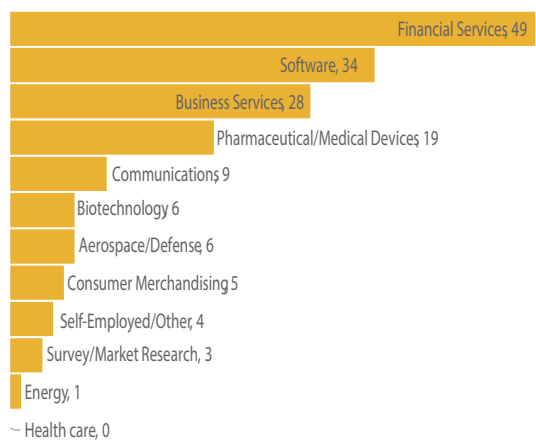

Looking at those employed by type of Business/Industry (164) we see that:

- $47 \%$ are US citizens.

- Females hold 32\% of BI positions; 51\% of these are US citizens and 23\% work in financial services.

- $6 \%$ hold temporary positions; almost all are held by non-US citizens and 10\% could not find a suitable permanent position.

- $14 \%$ of those employed in BI are currently looking form another position. 


\section{ANNUAL SURVEY}

Figure EE.7. Age Distribution of New PhD Respondents

- Male Female Neither

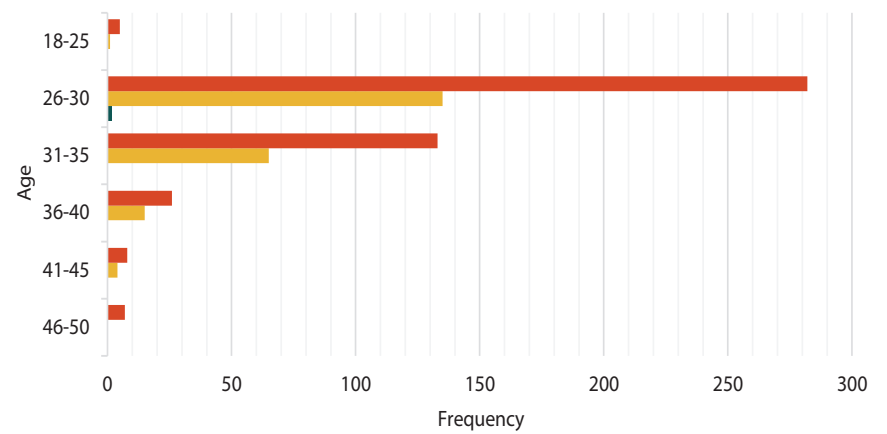

Figure EE. 7 gives the age distribution of the 683 new doctoral recipients who respond to this question. The median age of new doctoral recipients was 29 while the mean was 30.5 .

- The youngest new $\mathrm{PhD}$ recipient was 18 and the oldest was 50.

- $61 \%$ of all new $\mathrm{PhD}$ recipients are between the ages of 26-30

- The mode is 28 ( $21 \%$ of females and $18 \%$ of males reported being age 28 ).

\section{Starting Salaries of the 2015-2016 Doctoral Recipients}

The starting salary figures were compiled from information gathered on the EENDR questionnaires sent to 1,656 individuals using addresses provided by the departments granting the degrees; 730 individuals responded between late October 2016 and June 2017. Responses with insufficient data or from individuals who indicated they had part-time or non-US employment were excluded. Numbers of usable responses for each salary category are reported in the following tables.

Readers should be warned that the data in this report are obtained from a self-selected sample, and inferences from them may not be representative of the full population. Detailed information, including boxplots which traditionally appeared in this report, is available on the AMS website atwww.ams.org/annual-survey/survey-reports.

\section{Academic Teaching/Teaching and Research 9-10-Month Starting Salaries $\dagger$ (in thousands of dollars)}

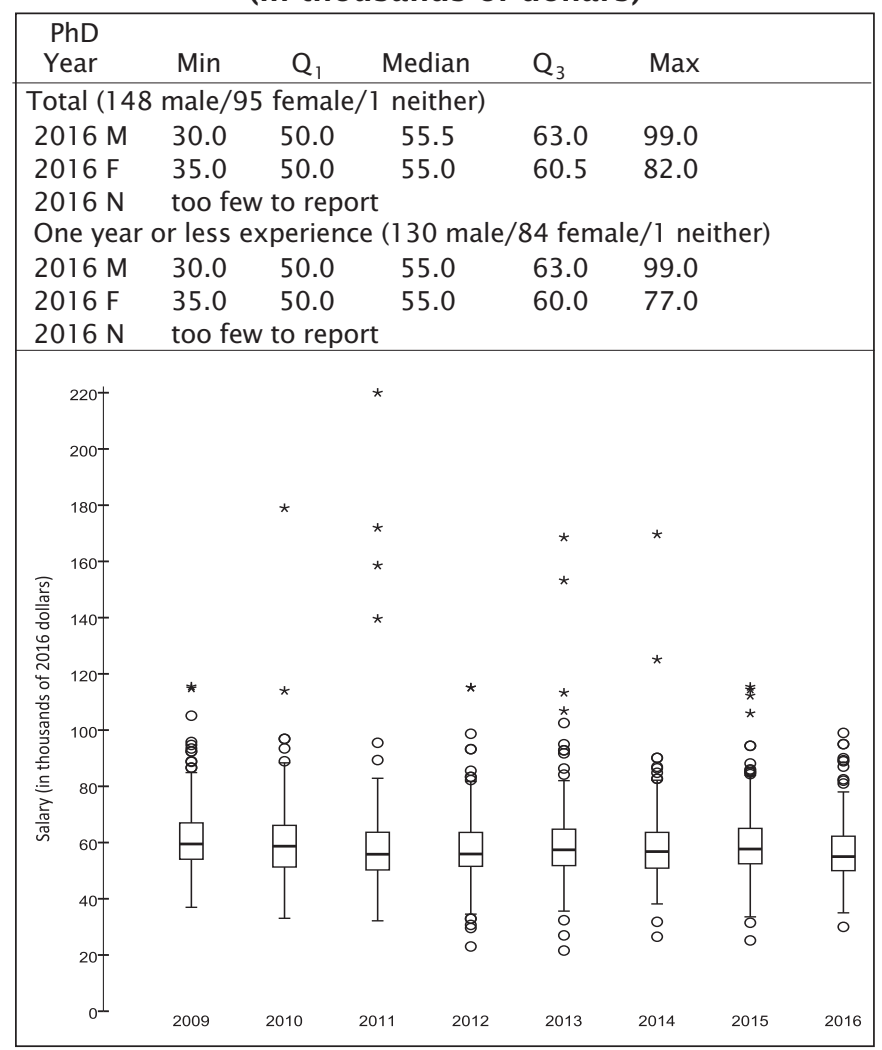

\footnotetext{
† Includes postdoctoral salaries.
}

\section{Academic Postdoctorates Only ${ }^{\dagger}$ \\ 9-10-Month Starting Salaries (in thousands of dollars)}

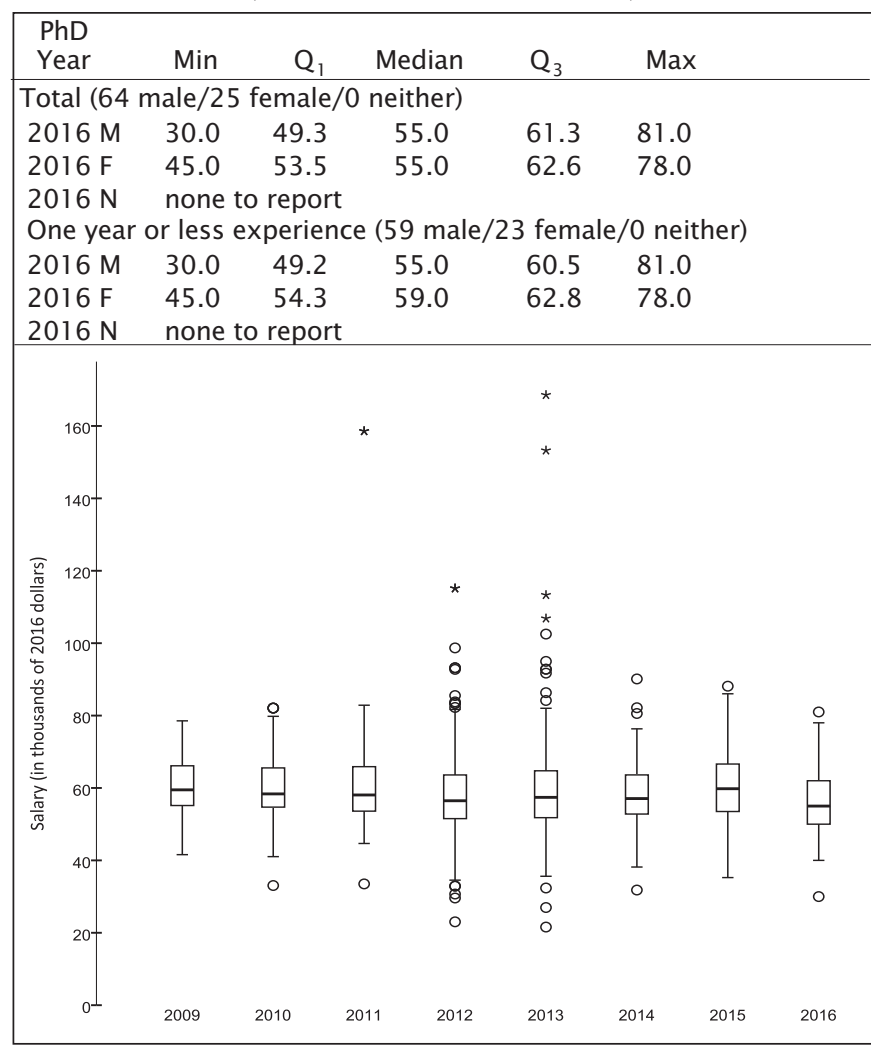

† A postdoctoral appointment is a temporary position primarily intended to provide an opportunity to extend graduate training or to further research experience. 


\section{ANNUAL SURVEY}

\section{Government \\ 11-12-Month Starting Salaries (in thousands of dollars)}

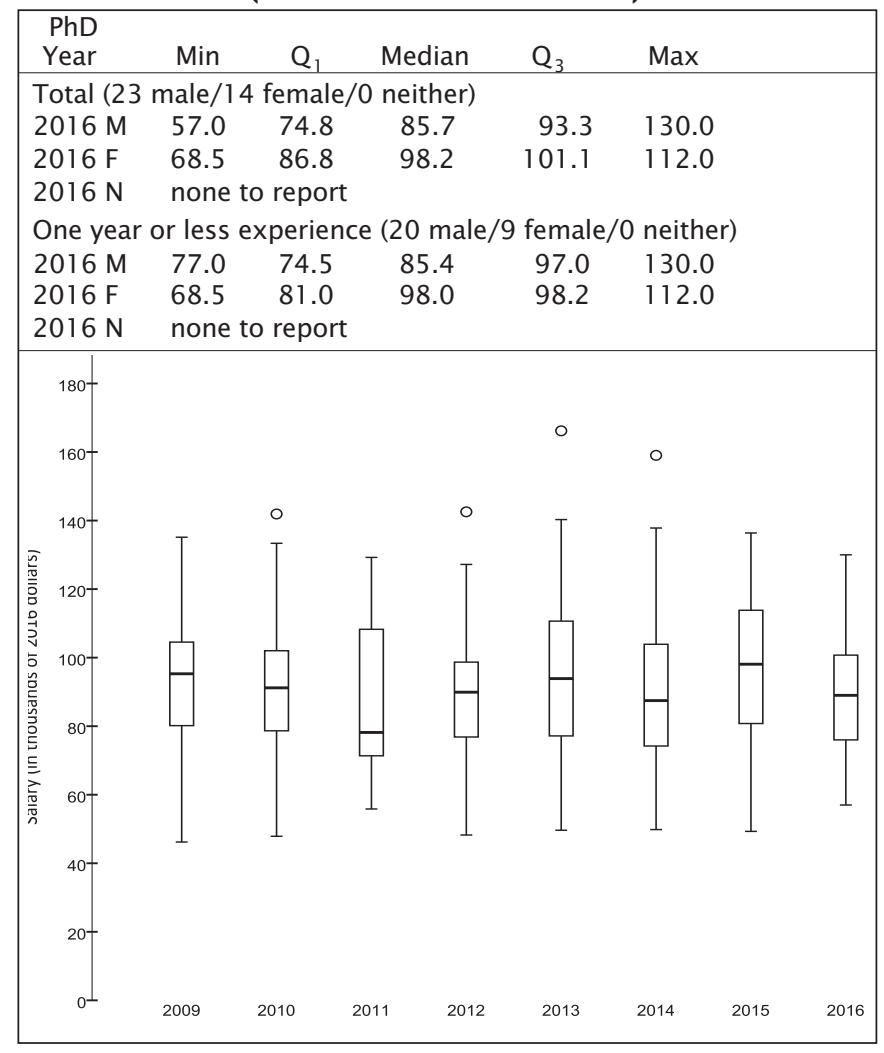

Business and Industry

11-12-Month Starting Salaries (in thousands of dollars)

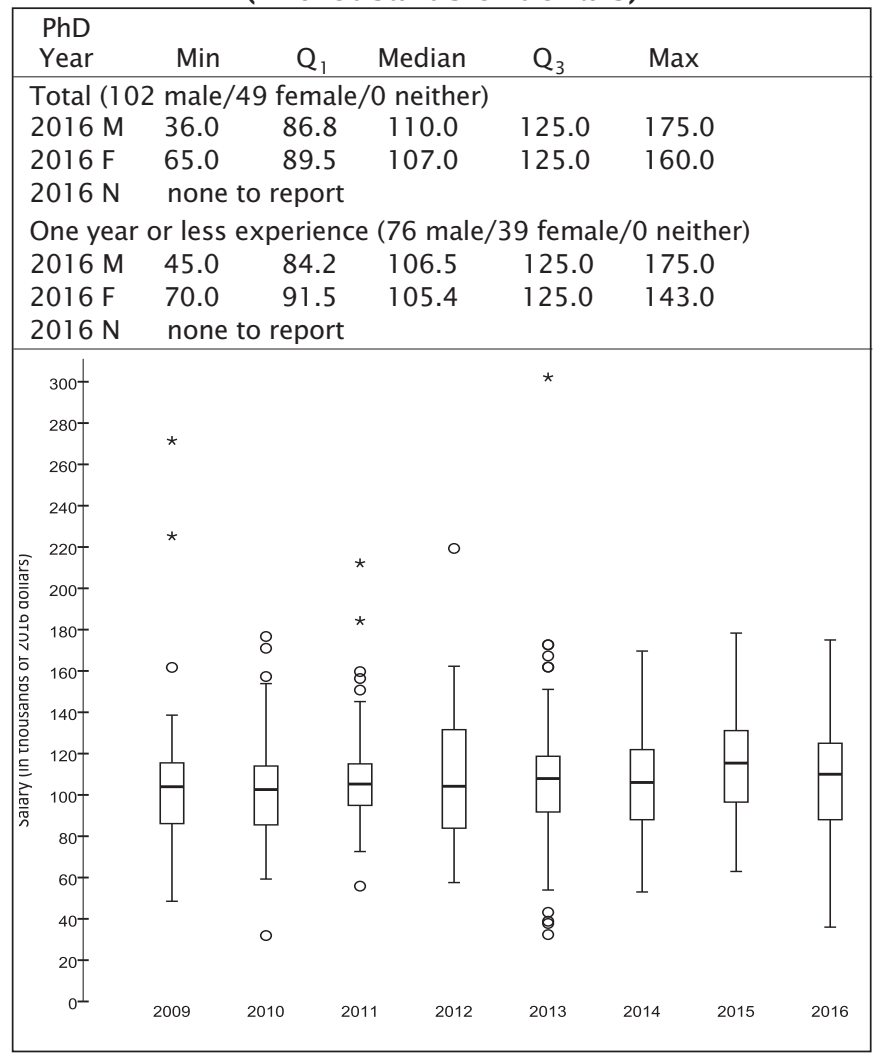

\section{Remarks on Starting Salaries}

Key to Tables and Graphs. Salaries are those reported for the fall immediately following the survey cycle. Years listed denote the survey cycle in which the doctorate was received-for example, survey cycle July 1, 2015-June 30, 2016 is designated as 2016. Salaries reported as 9-10 months exclude stipends for summer grants or summer teaching or the equivalent. $\mathrm{M}$ and $\mathrm{F}$ are male and female, respectively. Male and female figures are not provided when the number of salaries available for analysis in a particular category was five or fewer. All categories of "Teaching/Teaching and Research" and "Research Only" contain those recipients employed at academic institutions only.

Graphs. The graphs show standard boxplots summarizing salary distribution information for the years 2009 through 2016. Values plotted for 2009 through 2015 are converted to 2016 dollars using the implicit price deflator prepared annually by the Bureau of Economic Analysis, US Department of Commerce. These categories are based on work activities reported in EENDR. Salaries of postdoctorates are shown separately. They are also included in other academic categories with matching work activities.

For each boxplot the box shows the first quartile (Q1), the median (M), and the third quartile (Q3). Upper whiskers extend from $\mathrm{Q}_{3}$ to the largest data value below $\mathrm{Q}_{3}+1.5 \mathrm{IQR}$, and lower whiskers from $\mathrm{Q}_{1}$ down to the smallest data value above $\mathrm{Q}_{1}-1.5 \mathrm{IQR}$. Data points falling between $\mathrm{Q}_{3}+1.5 \mathrm{IQR}$ and $\mathrm{Q}_{3}+3 \mathrm{IQR}$ or $\mathrm{Q}_{1}-1.5 \mathrm{IQR}$ and $\mathrm{Q}_{3}-3 \mathrm{IQR}$ are designated as outliers and plotted as circles (o). Data outside the range $\mathrm{Q}_{1}-3 \mathrm{IQR}$ to $\mathrm{Q}_{3}+3 \mathrm{IQR}$ are designated as extreme outliers and plotted as stars (*). 


\section{ANNUAL SURVEY}

\section{Response Rates}

New PhD Recipient Response Rates by Granting Department Grouping

\begin{tabular}{|lcc|}
\hline Granting Department Group & Number & Percent \\
\hline Math Public Large & 152 of 348 & $44 \%$ \\
\hline Math Public Medium & 130 of 305 & $43 \%$ \\
\hline Math Public Small & 103 of 218 & $47 \%$ \\
\hline Math Private Large & 112 of 215 & $52 \%$ \\
\hline Math Private Small & 41 of 84 & $49 \%$ \\
\hline Applied Math & 51 of 97 & $53 \%$ \\
\hline Statistics & 82 of 246 & $33 \%$ \\
\hline Biostatistics & 59 of 143 & $41 \%$ \\
\hline Total & 730 of $\mathbf{1 6 5 6}$ & $\mathbf{4 4 \%}$ \\
\hline
\end{tabular}

Distribution of New PhD Recipient Responses by Employer Type

\begin{tabular}{|lcc|}
\hline Employer Type & Number & Percent \\
\hline Math Public Large & 49 & $7 \%$ \\
\hline Math Public Medium & 31 & $4 \%$ \\
\hline Math Public Small & 34 & $5 \%$ \\
\hline Math Private Large & 32 & $4 \%$ \\
\hline Math Private Small & 17 & $2 \%$ \\
\hline Applied Math & 7 & $1 \%$ \\
\hline Statistics & 11 & $2 \%$ \\
\hline Biostatistics & 16 & $2 \%$ \\
\hline Masters & 33 & $5 \%$ \\
\hline Bachelors & 96 & $13 \%$ \\
\hline Two-Year institutions & 14 & $2 \%$ \\
\hline Other Academic & 57 & $8 \%$ \\
\hline Research Institute/Other Non-profit & 33 & $5 \%$ \\
\hline Government & 37 & $5 \%$ \\
\hline Business/Industry & 157 & $22 \%$ \\
\hline Non-US Academic & 66 & $9 \%$ \\
\hline Non-US Nonacademic & 8 & $1 \%$ \\
\hline Not Seeking (US) & 5 & $1 \%$ \\
\hline Still Seeking (US) & 18 & $2 \%$ \\
\hline Unknown (US) & 1 & $0 \%$ \\
\hline Non-US: Not seeking, Still seeking, Unknown & 8 & $1 \%$ \\
\hline Total & 730 & $\mathbf{1 0 0 \%}$ \\
\hline \hline
\end{tabular}

\section{Acknowledgments}

The Annual Survey attempts to provide an accurate appraisal and analysis of various aspects of the academic mathematical sciences scene for the use and benefit of the community and for filling the information needs of the professional organizations. Every year, college and university departments in the United States are invited to respond. The Annual Survey relies heavily on the conscientious efforts of the dedicated staff members of these departments for the quality of its information. On behalf of the Data Committee and the Annual Survey Staff, we thank the many secretarial and administrative staff members in the mathematical sciences departments for their cooperation and assistance in responding to the survey questionnaires. For this EENDR report, we thank the PhD recipients responded to the survey. Their participation is vital to our provided accurate and timely information.

The Annual Survey is co-sponsored by the American Mathematical Society (AMS), the American Statistical Association (ASA), Institute for Mathematical Statistics (IMS), Mathematical Association of America (MAA), and the Society for Industrial and Applied Mathematics (SIAM). 


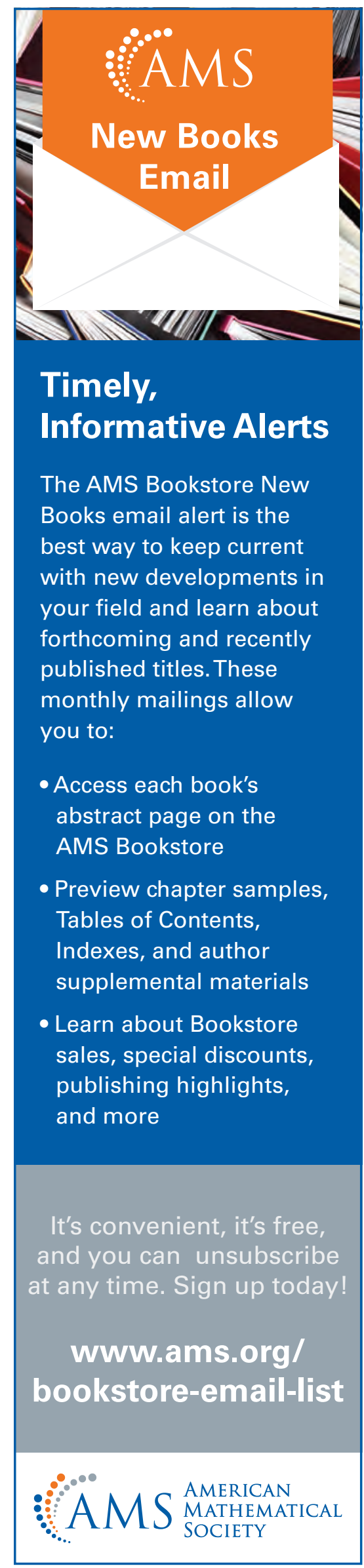

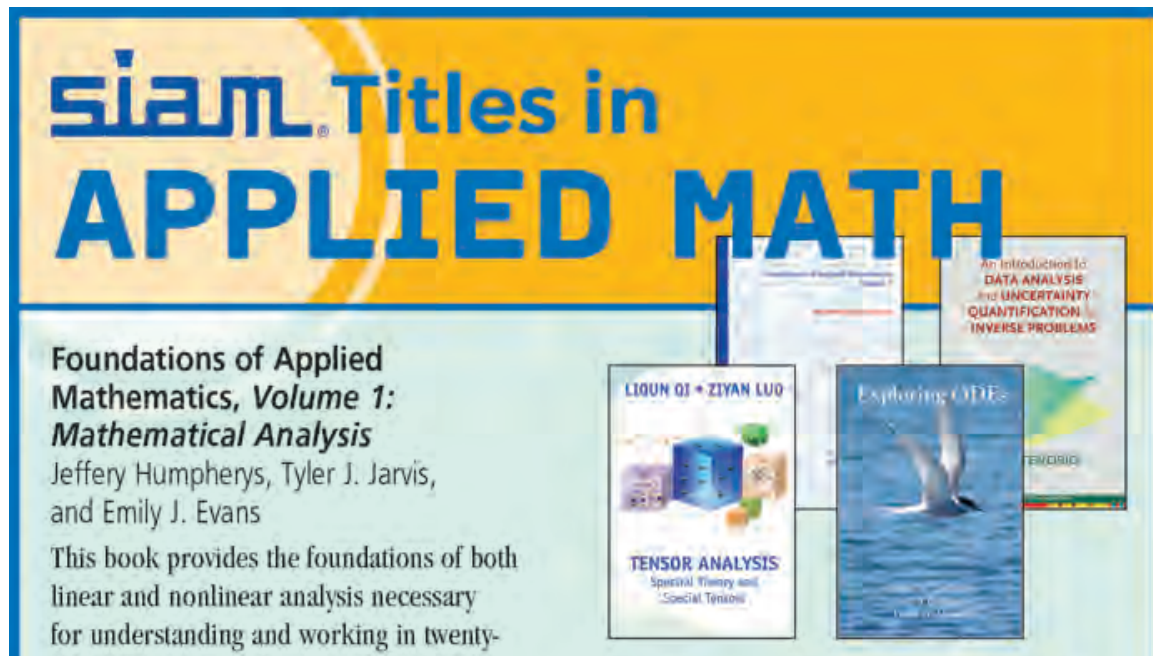

first century applied and computational mathematics.

$2017 \cdot x x+689$ pages $\cdot$ Hardcover $\cdot 978-1-611974-89-8$

List $\$ 89.00 \cdot$ SIAM Member $\$ 62.30 \cdot 0 T 152$

\section{An Introduction to Data Analysis and Uncertainty Quantification for Inverse Problems \\ Luis Tenorio \\ Matbematics in Industry 03}

The author covers basic statistical inference, introduces the framework of ill-posed inverse problems, and explains stalistical questions that arise in their applications.

$2017 \cdot x+269$ pages - Softcover $\cdot 978-1-611974-91-1$ List $\$ 69.00 \cdot$ SIAM Member $\$ 48.30 \cdot$ MN03

\section{Fundamentals of Numerical Computation}

Tobin A. Driscoll and Richard J. Braun An advanced undergraduate-level introduction to the mathematics and use of algorithms for the fundamental problems of numerical computation: linear algebra, finding roots, approximating data and functions, and solving differential equations.

$2017 \cdot x x x+553$ pages - Hardcover - 978-1-611975-07.9 List $\$ 104.00$ - Member $\$ 72.80$ - OT154

\section{Exploring ODEs}

Lloyd N. Trefethen, Ásgeir Birkisson, and Tobin A. Driscoll

A textbook of ordinary differential equations for advanced undergraduates, graduate students, scientists, and engineers, with each concept illustrated numerically via a few lines of Chebfun code.

2018 , viii +335 pages, Hardcover $\cdot 978-1-611975-15-4$ List $\$ 64.00$. SIAM Member $\$ 44.80 \cdot 0157$

\section{Tensor Analysis: Spectral Theory and Special Tensors}

Liqun Qi and Ziyan Luo

The first book on spectral theory of tensors, the theory of special tensors, and the spectral hypergraph theory via tensors.

$2017 \cdot x^{\prime}+305$ pages - Softcover - 978-1-611974-744 List $\$ 84.00$ - SIAM Member $\$ 58.80 \cdot$ OT151

\section{First-Order Methods in Optimization} Amir Beck

MOS-SIAM Series on Optimization 25

A self contained, comprehensive study of the main first-order methods that are frequently used in solving large-scale problems.

$2017 \cdot x+484$ pages - Softcover $\cdot 978-1-611974.98-0$ List $\$ 97.00$ - SIAM Member $\$ 67.90 \cdot$ MO25

\section{MATLAB Guide, Third Edition}

Desmond J. Higham and Nicholas J. Higham This third edition of MATZAB Guide completely revises and updates the best-selling second edition and is more than 25 percent longer. 2017 - xxvi + 476 pages - Hardcover - 978-1-61 1974-65-2 List $\$ 62.00$ - SLAM Member $\$ 43.40 \cdot$ OT150

\section{Learning LaTeX, Second Edition}

David F. Griffiths and Desmond F. Higham

"I never would have thought it possible, but the new edition is a substantial improvement with the additional coverage of BiBTeX, Beamer, and posters. Learning LaTeX should be handed to new graduate students in mathematical sciences along with their office key and ID card."

- Don Estep, Colorado State University $2016 \cdot x+103$ pages $\cdot$ Softcover $\cdot 978-1-611974-41-6$ List $\$ 29.00 \cdot$ SLAM Member $\$ 20.30 \cdot$ OT148

\section{ORDER AT BOOKSTORE.SIAM.ORG}

All prices are in US dollars.

Use your credit card (AMEX. MasterCard, and VISA) by phone: $+1-215-382-9800$ worldwide or toll free at 800-447-SIAM in USA and Canada. Or send check or money order in US dollars to: SIAM, Dept. BKNO18. 3600 Market Street. 6th Floor. Philadelphia. PA 19104-2688 USA. Outside North America, buy SIAM books through the Eurospan Group via eurospanbookstore.com/siam. 


\section{New Publications Offered by the AMS}

To subscribe to email notification of new AMS publications, please go to www. ams . org/bookstore-emai 1.

\section{Geometry and Topology}

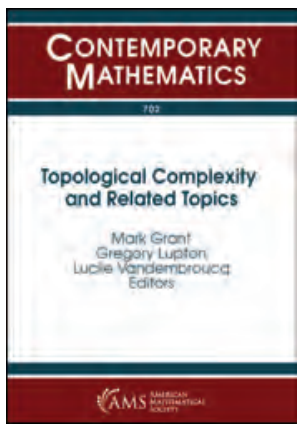

\section{Topological \\ Complexity and Related Topics}
Mark Grant, University of Aberdeen, United Kingdom, Gregory Lupton, Cleveland State University, $\mathrm{OH}$, and Lucile Vandembroucq, University of Minho, Braga, Portugal, Editors

This volume contains the proceedings of the mini-workshop on Topological Complexity and Related Topics, held from February 28-March 5, 2016, at the Mathematisches Forschungsinstitut Oberwolfach.

Topological complexity is a numerical homotopy invariant, defined by Farber in the early twenty-first century as part of a topological approach to the motion planning problem in robotics. It continues to be the subject of intensive research by homotopy theorists, partly due to its potential applicability, and partly due to its close relationship to more classical invariants, such as the Lusternik-Schnirelmann category and the Schwarz genus.

This volume contains survey articles and original research papers on topological complexity and its many generalizations and variants, to give a snapshot of contemporary research on this exciting topic at the interface of pure mathematics and engineering.

This item will also be of interest to those working in analysis.

Contents: Survey Articles: A. Ángel and H. Colman, Equivariant topological complexities; J. Carrasquel, Rational methods applied to sectional category and topological complexity; D. C. Cohen, Topological complexity of classical configuration spaces and related objects; P. Pavešić, A topologist's view of kinematic maps and manipulation complexity; Research Articles: D. M. Davis, On the cohomology classes of planar polygon spaces; J.P. Doeraene, M. El Haouari, and C. Ribeiro, Sectional category of a class of maps; L. Fernández Suárez and L. Vandembroucq, Q-topological complexity; N. Fieldsteel, Topological complexity of graphic arrangements; J. González, M. Grant, and L. Vandembroucq, Hopf invariants, topological complexity, and LS-category of the cofiber of the diagonal map for two-cell complexes; J. González and B. Gutiérrez, Topological complexity of collision-free multi-tasking motion planning on orientable surfaces; M. Grant and D. Recio-Mitter, Topological complexity of subgroups of Artin's braid groups.

Contemporary Mathematics, Volume 702

March 2018, 176 pages, Softcover, ISBN: 978-1-4704-3436-6, LC 2017042708, 2010 Mathematics Subject Classification: 55-06, 20F36, 52C35, 55M30, 55P62, 55P91, 55Q25, 57M15, 68T40, 93C85, AMS members US\$93.60, List US\$117, Order code CONM/702

\section{Number Theory}

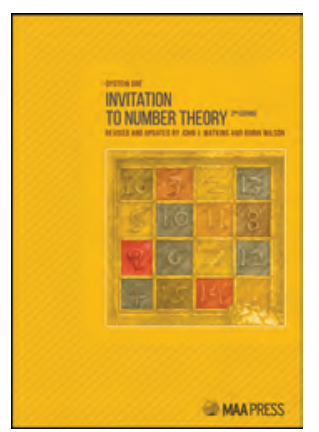

\section{Invitation to Number Theory}

Second Edition

Oystein Ore

Revised and updated by John J. Watkins and Robin Wilson

Number theory is the branch of mathematics concerned with the counting numbers, 1, 2, 3, .. and their multiples and factors. Of particular importance are odd and even numbers, squares and cubes, and prime numbers. But in spite of their simplicity, readers will meet a multitude of topics in this book: magic squares, cryptarithms, finding the day of the week for a given date, constructing regular polygons, pythagorean triples, and many more.

In this revised edition, John Watkins and Robin Wilson have updated the text to bring it in line with contemporary developments, added new material on Fermat's Last Theorem, the role of computers in number theory, and the use of number theory in cryptography, and have made numerous minor changes in the presentation and layout of the text and the exercises.

MAA Press: An imprint of the American Mathematical Society Anneli Lax New Mathematical Library, Volume 49

February 2018, 134 pages, Softcover, ISBN: 978-0-88385-653-6, LC 2017954601, AMS members US\$38.40, List US\$48, Order code NML/49 


\section{New AMS-Distributed Publications}

\section{Algebra and Algebraic Geometry}

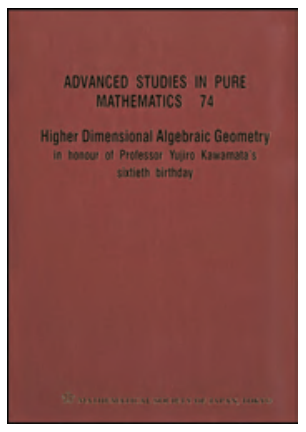

\section{Higher Dimensional Algebraic Geometry}

In Honor of Professor

Yujiro Kawamata's 60th Birthday

Keiji Oguiso, University of Tokyo, Komaba Meguro-ku, Japan, Caucher Birkar, University of Cambridge, United Kingdom, Shihoko Ishii, Tokyo Woman's Christian University, Japan, and Shigeharu Takayama, University of Tokyo, Japan, Editors

This volume is the proceedings of the conference "Higher Dimensional Algebraic Geometry", in honor of Professor Yujiro Kawamata's 60th birthday.

This volume consists of 20 inspiring research papers on birational algebraic geometry, minimal model program, derived algebraic geometry, classification of algebraic varieties, and transcendental methods by very active top level mathematicians from all over the world. This volume will be useful for researchers in these areas as well as those who are studying algebraic geometry.

Published for the Mathematical Society of Japan by Kinokuniya, Tokyo, and distributed worldwide, except in Japan, by the AMS.

Advanced Studies in Pure Mathematics, Volume 74

November 2017, 437 pages, Hardcover, ISBN: 978-4-86497-046- 4, 2010 Mathematics Subject Classification: 14J10; 14J15, AMS members US\$66.40, List US\$83, Order code ASPM/74

\section{CMS Winter Meeting}

December 7-10, 2018

Sheraton Vancouver Wall Centre, Vancouver, British Columbia

\section{CALL FOR SESSIONS}

\section{Deadline: March 31, 2018}

The Canadian Mathematical Society (CMS) welcomes and invites session proposals for the 2018 CMS Winter Meeting in Vancouver from December 7 - 10, 2018. Proposals should include a brief description of the focus and purpose of the session, the expected number of speakers, as well as the organizer's name, complete address, telephone number, e-mail address, etc. Sessions will be advertised in the CMS Notes, on the web site and in the AMS Notices. Speakers will be requested to submit abstracts, which will be published on the web site and in the meeting program. Those wishing to organize a session should send a proposal to the Scientific Directors.

\section{Scientific Directors:}

Franco Saliola (UQAM)

saliola.franco@uqam.ca

Malabika Pramanik (UBC) malabika@math.ubc.ca

\section{Réunion d'hiver de la SMC 2018}

7-10 décembre 2018

Sheraton Vancouver Wall Centre, Vancouver, Colombie Britannique

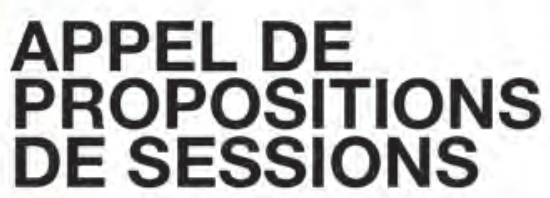

Date limite : $\mathbf{3 1}$ mars 2018

La Société mathématique du Canada (SMC) vous invite à proposer des sessions pour la Réunion d'hiver de la SMC qui aura lieu à Vancouver du 7 au 10 décembre 2018. Ces propositions doivent présenter une brève description de l'orientation et des objectifs de la session, le nombre de conférenciers prévu, de même que le nom l'adresse complète, le numéro de téléphone et l'adresse électronique de l'organisateur. Toutes les sessions seront annoncées dans les Notes de la SMC, sur le site Web et dans les notices de I'AMS. Les conférenciers devront présenter un résumé, qui sera publié sur le site Web et dans le programme de la réunion. Toute personne qui souhaiterait organiser une session est priée de faire parvenir une proposition à un des directeurs scientifique.

\section{Directeur scientifique:}

Franco Saliola (UQAM) saliola.franco@uqam.ca

Malabika Pramanik (UBC) malabika@math.ubc.ca 


\section{Classified Advertising}

\section{Positions available, items for sale, services available, and more}

\section{CHINA \\ Tianjin University, China \\ Tenured/Tenure-Track/Postdoctoral Positions at the Center for Applied Mathematics}

Dozens of positions at all levels are available at the recently founded Center for Applied Mathematics, Tianjin University, China. We welcome applicants with backgrounds in pure mathematics, applied mathematics, statistics, computer science, bioinformatics, and other related fields. We also welcome applicants who are interested in practical projects with industries. Despite its name attached with an accent of applied mathematics, we also aim to create a strong presence of pure mathematics. Chinese citizenship is not required.

Light or no teaching load, adequate facilities, spacious office environment and strong research support. We are prepared to make quick and competitive offers to self-motivated hard workers, and to potential stars, rising stars, as well as shining stars.

The Center for Applied Mathematics, also known as the Tianjin Center for Applied Mathematics (TCAM), located by a lake in the central campus in a building protected as historical architecture, is jointly sponsored by the Tianjin municipal government and the university. The initiative to establish this center was taken by Professor S. S. Chern. Professor Molin Ge is the Honorary Director, Professor Zhiming $\mathrm{Ma}$ is the Director of the Advisory Board. Professor William Y. C. Chen serves as the Director.

TCAM plans to fill in fifty or more permanent faculty positions in the next few years. In addition, there are a number of temporary and visiting positions. We look forward to receiving your application or inquiry at any time. There are no deadlines.

Please send your resume to mathjobs@ tju.edu.cn. For more information, please visit cam.tju.edu.cn or contact Ms. Erica Liu at mathjobs@ tju.edu. cn, telephone: 86-22-2740-6039.

\author{
KOREA \\ KOREA INSTITUTE FOR ADVANCED \\ STUDY (KIAS) Assistant Professor \& \\ Research Fellow in Pure and Applied \\ Mathematics
}

The School of Mathematics at the Korea Institute for Advanced Study (KIAS) invites applicants for the positions at the level of KIAS Assistant Professor and Postdoctoral Research Fellow in pure and applied mathematics.

KIAS, founded in 1996, is committed to the excellence of research in basic sciences (mathematics, theoretical physics, and computational sciences) through high-quality research programs and a strong faculty body consisting of distinguished scientists and visiting scholars.

Applicants are expected to have demonstrated exceptional research potential, including major contributions beyond or through the doctoral dissertation.

Suggested uses for classified advertising are positions available, books or lecture notes for sale, books being sought, exchange or rental of houses, and typing services. The publisher reserves the right to reject any advertising not in keeping with the publication's standards. Acceptance shall not be construed as approval of the accuracy or the legality of any advertising.

The 2018 rate is $\$ 3.50$ per word with a minimum two-line headline. No discounts for multiple ads or the same ad in consecutive issues. For an additional $\$ 10$ charge, announcements can be placed anonymously. Correspondence will be forwarded.

Advertisements in the "Positions Available" classified section will be set with a minimum one-line headline, consisting of the institution name above body copy, unless additional headline copy is specified by the advertiser. Headlines will be centered in boldface at no extra charge. Ads will appear in the language in which they are submitted.

There are no member discounts for classified ads. Dictation over the telephone will not be accepted for classified ads.

Upcoming deadlines for classified advertising are as follows: April 2018-January 30, 2018; May 2018-March 2, 2018; June/July 2018-April 27, 2018; August 2018-June 6, 2018; June/July 2018-May 16, 2018.

US laws prohibit discrimination in employment on the basis of color, age, sex, race, religion, or national origin. "Positions Available" advertisements from institutions outside the US cannot be published unless they are accompanied by a statement that the institution does not discriminate on these grounds whether or not it is subject to US laws. Details and specific wording may be found on page 1373 (vol. 44).

Situations wanted advertisements from involuntarily unemployed mathematicians are accepted under certain conditions for free publication. Call toll-free 800-321-4AMS (321-4267) in the US and Canada or 401-455-4084 worldwide for further information.

Submission: Promotions Department, AMS, P.O. Box 6248, Providence, Rhode Island 02904; or via fax: 401-331-3842; or send email to c7assads@ams .org. AMS location for express delivery packages is 201 Charles Street, Providence, Rhode Island 02904. Advertisers will be billed upon publication. 
The annual salary starts from 50,000,000 Korean Won (approximately US $\$ 45,000$ at current exchange rate) for Research Fellows, and 57,000,000 Korean Won for KIAS Assistant Professors, respectively. In addition, individual research funds of 10,000,000 $\sim$ 13,000,000 Korean Won are available per year. The initial appointment for the position is for two years and is renewable for up to two additional years, depending on research performance and the needs of the research program at KIAS.

Applications will be reviewed twice a year, May 20 and November 20, and selected applicants will be notified in a month after the review. In exceptional cases, applications can be reviewed other times based on the availability of positions. The starting date of the appointment is negotiable. Applications must include a complete vitae with a cover letter, a list of publications, a research plan, and three letters of recommendation (All documents should be in English).

All should be sent by post or e-mail to:

Ms. Sojung Bae (mathkias@kias.re.kr)

School of Mathematics, Korea Institute for Advanced Study (KIAS), 85 Hoegiro (Cheongnyangni-dong 207-43), Dongdaemun-gu, Seoul 02455, Republic of Korea.

\section{Moving?}

Please make sure that the AMS Notices and Bulletin find their new home.

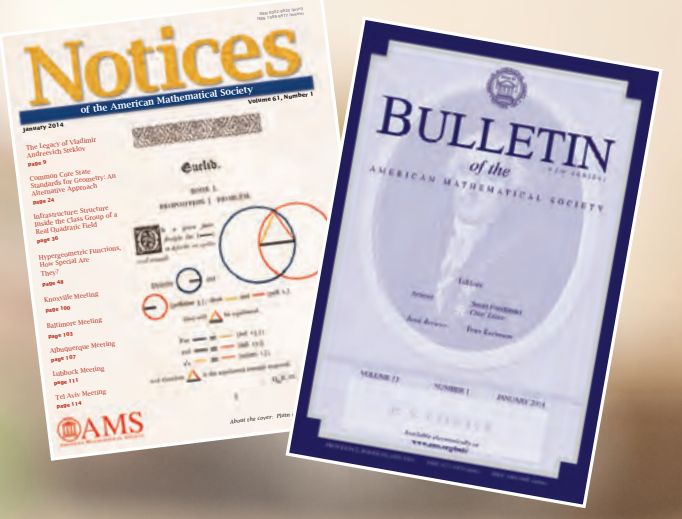

- Email your new address to us: amsmem@ams.org

- or make the change yourself online at: www.ams.org/cml-update

- or send the information to:

Member and Customer Services American Mathematical Society 201 Charles Street

Providence, RI 02904-2213 USA Phone: (800) 321-4267 (US \& Canada) (401) 455-4000 (Worldwide) 


\section{MathSciNet Mathematical Reviews}

WWW.ams.org/mathscinet

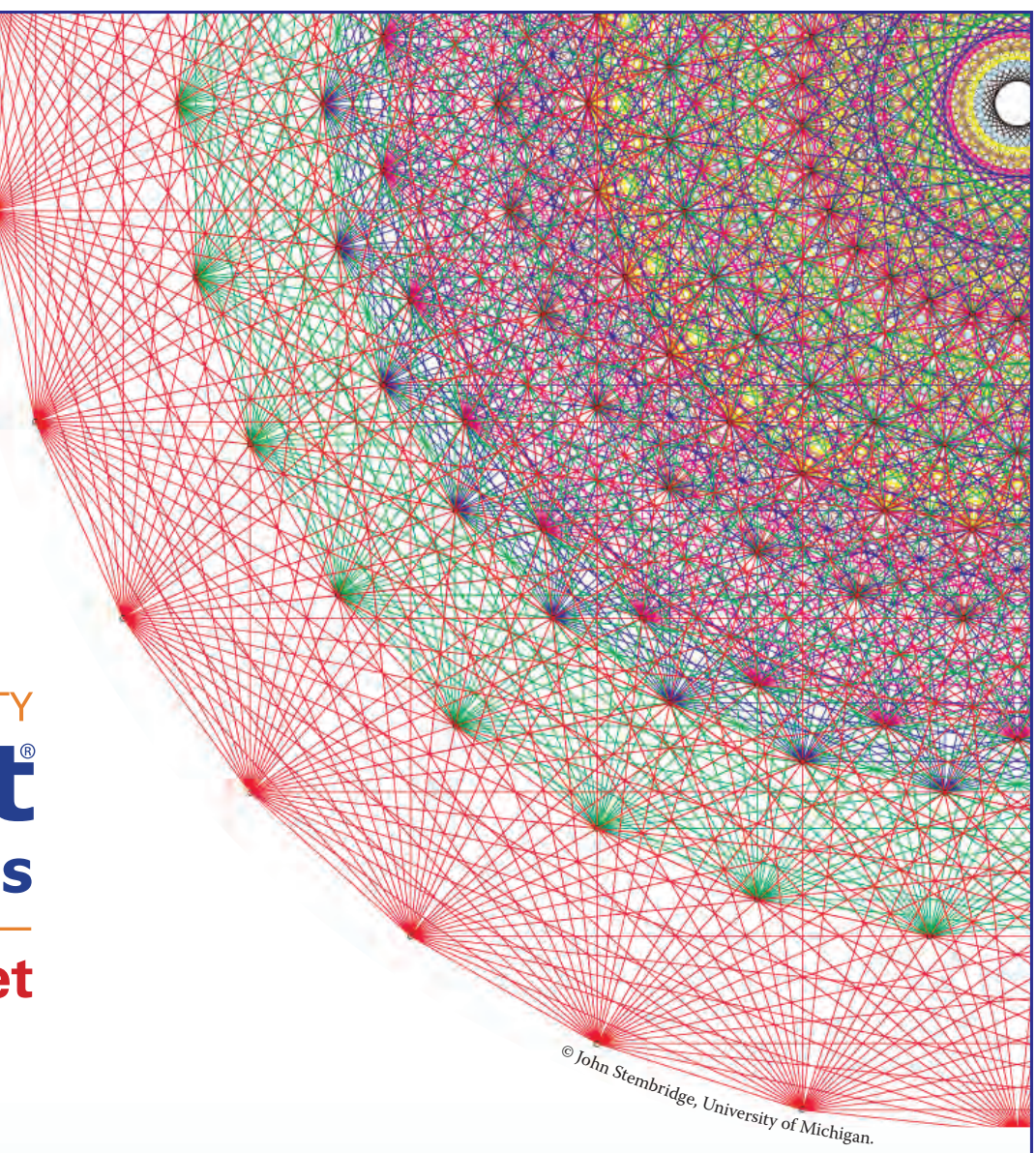

\section{MathSciNet is the mod complete and reliable source for your research}

Mathematical Reviews/MathSciNet, compiled, edited, and delivered by the AMS, is the authoritative gateway to the scholarly literature of mathematics. MathSciNet contains information on over 3 million articles and books, with direct links to almost 2 million articles in over 1,800 journals. MathSciNet includes expert reviews, personalizable author profiles, and citation information on articles, books, journals, and authors.

MathSciNet's extensive resources can help you both in your graduate research and throughout your math career. Use it to:

- Quickly get up to speed on a new topic

- Look up a researcher's body of work (and find his/her Erdős number!)

- Find an article or book and track its reference list

- Research a math department to prepare for a job interview

\section{Howto-Subscribe/Purchase}

Go to www.ams.org/mathsciprice to learn more about MathSciNet, including information about joining a consortium, subscription rates, and a 30-day free trial.

\footnotetext{
$\because \because A$ A $\because \begin{aligned} & \text { AMERICAN } \\ & \text { MATHEMATICAL } \\ & \text { SOCIETY }\end{aligned}$
} 


\section{MEETINGS \& CONFERENCES OF THE AMS}

\section{MARCH TABLE OF CONTENTS}

The Meetings and Conferences section of the Notices gives information on all AMS meetings and conferences approved by press time for this issue. Please refer to the page numbers cited on this page for more detailed information on each event. Invited Speakers and Special Sessions are listed as soon as they are approved by the cognizant program committee; the codes listed are needed for electronic abstract submission. For some meetings the list may be incomplete. Information in this issue may be dated.
The most up-to-date meeting and conference information can be found online at: Www.ams.org/meetings/.

Important Information About AMS Meetings: Potential organizers, speakers, and hosts should refer to page 88 in the January 2018 issue of the Notices for general information regarding participation in AMS meetings and conferences.

Abstracts: Speakers should submit abstracts on the easy-to-use interactive Web form. No knowledge of LTEX is necessary to submit an electronic form, although those who use IATEX may submit abstracts with such coding, and all math displays and similarily coded material (such as accent marks in text) must be typeset in LTEX. Visit www.ams.org/ cgi-bin/abstracts/abstract.pl/. Questions about abstracts may be sent to absinfo@ams.org. Close attention should be paid to specified deadlines in this issue. Unfortunately, late abstracts cannot be accommodated.

\section{MEETINGS IN THIS ISSUE}

\section{8}

$\begin{array}{lll}\text { March 17-18 } & \text { Columbus, Ohio } & \text { p. } 373 \\ \text { April 14-15 } & \text { Nashville, Tennesse } & \text { p. } 374 \\ \text { April 14-15 } & \text { Portland, Oregon } & \text { p. } 375 \\ \text { April 21-22 } & \text { Boston, Massachusetts } & \text { p. } 376 \\ \text { June 11-14 } & \text { Shanghai, People's Republic } & \\ & \text { of China } & \text { p. } 378 \\ \text { September 29-30 } & \text { Newark, Delaware } & \text { p. } 378 \\ \text { October 20-21 } & \text { Ann Arbor, Michigan } & \text { p. } 379 \\ \text { October 27-28 } & \text { San Francisco, California } & \text { p. } 379 \\ \text { November 3-4 } & \text { Fayetteville, Arkansas } & \text { p. } 380\end{array}$

\section{9}

January 16-19

Baltimore, Maryland

p. 380

March 15-17

March 22-24

April 13-14

June 10-13

October 12-13

Auburn, Alabama

p. 381

Honolulu, Hawaii

p. 381

Hartford, Connecticut

p. 381

Quy Nhon City, Vietnam p. 381

Binghamton, New York

p. 382

November 2-3

Gainesville, Florida

p. 382

2020

January 15-18 Denver, Colorado

p. 382

\section{1}

January 6-9 Washington, DC

p. 382

See www.ams.org/meetings/ for the most up-to-date information on the meetings and conferences that we offer.

\section{ASSOCIATE SECRETARIES OF THE AMS}

Central Section: Georgia Benkart, University of WisconsinMadison, Department of Mathematics, 480 Lincoln Drive, Madison, WI 53706-1388; email: benkart@math.wisc.edu; telephone: 608-263-4283.

Eastern Section: Steven H. Weintraub, Department of Mathematics, Lehigh University, Bethlehem, PA 18015-3174; email: steve.weintraub@lehigh.edu; telephone: 610-758-3717.
Southeastern Section: Brian D. Boe, Department of Mathematics, University of Georgia, 220 D W Brooks Drive, Athens, GA 30602-7403, email: brian@math.uga.edu; telephone: 706-5422547.

Western Section: Michel L. Lapidus, Department of Mathematics, University of California, Surge Bldg., Riverside, CA 925210135; email: lapidus@math.ucr.edu; telephone: 951-827-5910. 


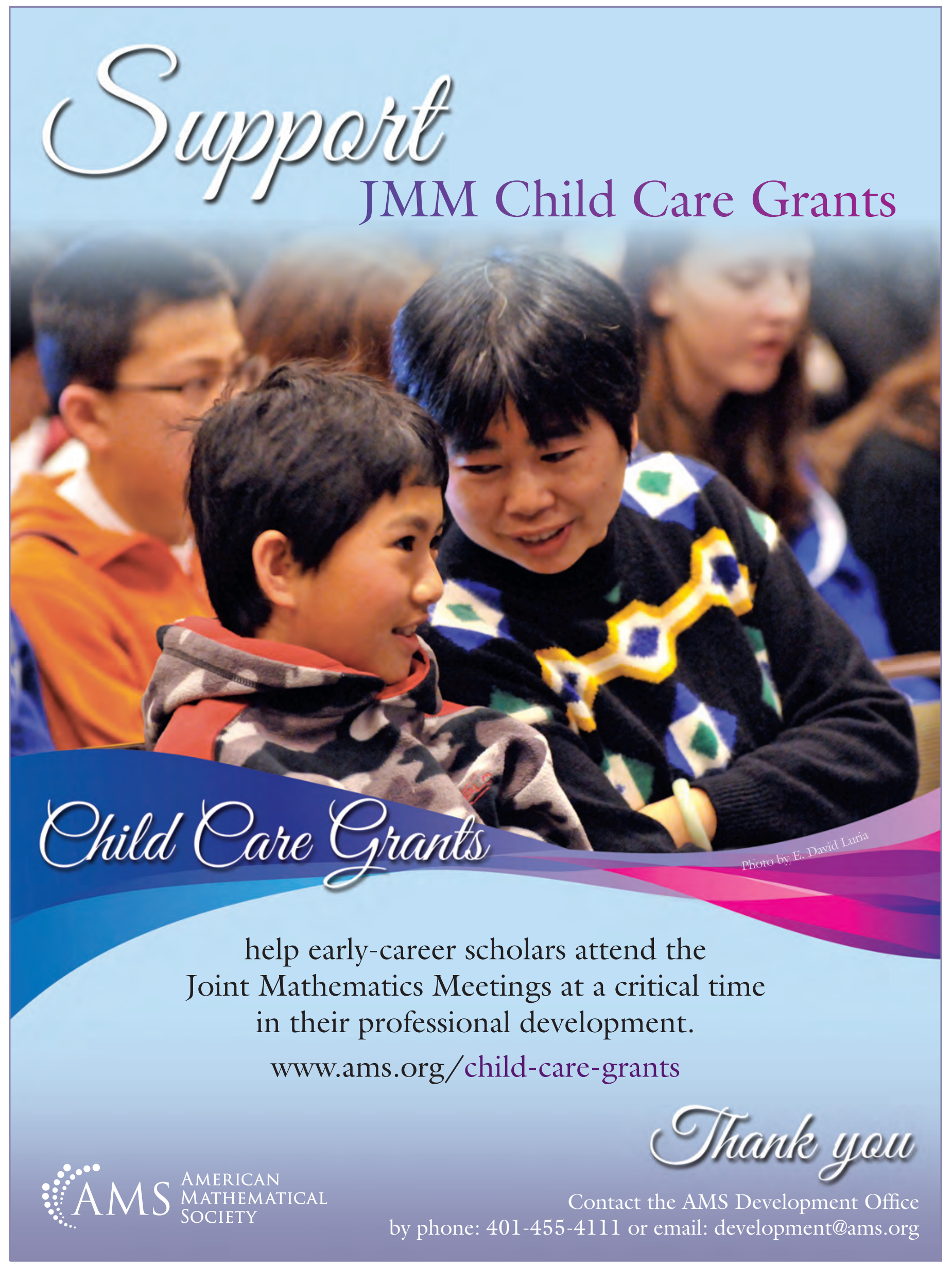




\section{Meetings \& Conferences of the AMS}

IMPORTANT INFORMATION REGARDING MEETINGS PROGRAMS: AMS Sectional Meeting programs do not appear

in the print version of the Notices. However, comprehensive and continually updated meeting and program informa-

tion with links to the abstract for each talk can be found on the AMS website. See www. ams. org/meetings/

Final programs for Sectional Meetings will be archived on the AMS website accessible from the stated URL

\section{Columbus, Ohio}

\section{Ohio State University}

\section{March 17-18,2018}

Saturday - Sunday

\section{Meeting \# 1136}

Central Section

Associate secretary: Georgia Benkart

Announcement issue of Notices: December 2017

Program first available on AMS website: January 31, 2018

Issue of Abstracts: Volume 39, Issue 2

\section{Deadlines}

For organizers: Expired

For abstracts: Expired

The scientific information listed below may be dated. For the latest information, see www.ams.org/amsmtgs/ sectional.htm1.

\section{Invited Addresses}

Aaron Brown, University of Chicago, Recent progress in the Zimmer program.

Tullia Dymarz, University of Wisconsin-Madison, $\mathrm{Bi}^{-}$ Lipschitz vs Quasi-isometric equivalence.

June Huh, Institute for Advanced Study, The correlation constant of a field.

\section{Special Sessions}

If you are volunteering to speak in a Special Session, you should send your abstract as early as possible via the abstract submission form found at www . ams . org/cgi-bin/ abstracts/abstract.p1.

Advances in Integral and Differential Equations, Jeffrey T. Neugebauer, Eastern Kentucky University, and Min Wang, Rowan University.
Algebraic Coding Theory and Applications, Heide Gluesing-Luerssen, University of Kentucky, Christine A. Kelley, University of Nebraska-Lincoln, and Steve Szabo, Eastern Kentucky University.

Algebraic Combinatorics: Association Schemes, Finite Geometry, and Related Topics, Sung Y. Song, Iowa State University, and Bangteng Xu, Eastern Kentucky University.

Algebraic Curves and Their Applications, Artur Elezi, American University, Monika Polak, Maria CurieSklodowska University (Poland) and University of Information Science and Technology (Mac, and Tony Shaska, Oakland University.

Algebraic and Combinatorial Aspects of Tropical Geometry, Maria Angelica Cueto, Ohio State University, Yoav Len, University of Waterloo, and Martin Ulirsch, University of Michigan.

Algebraic, Combinatorial, and Quantum Invariants of Knots and Manifolds, Cody Armond, Ohio State University, Mansfield, Micah Chrisman, Monmouth University, and Heather Dye, McKendree University.

Analytical and Computational Advances in Mathematical Biology Across Scales, Veronica Ciocanel and Alexandria Volkening, Mathematical Biosciences Institute.

Categorical, Homological and Combinatorial Methods in Algebra (Celebrating the 80th birthday of S. K. Jain), Pedro A. Guil Asensio, University of Murcia, Ivo Herzog, Ohio State University, Andre Leroy, University of Artois, and Ashish K. Srivastava, Saint Louis University.

Coherent Structures in Interfacial Flows, Benjamin Akers and Jonah Reeger, Air Force Institute of Technology.

Commutative and Combinatorial Algebra, Jennifer Biermann, Hobart and William Smith Colleges, and Kuei-Nuan Lin, Penn State University, Greater Allegheny.

Convex Bodies in Algebraic Geometry and Representation Theory, Dave Anderson, Ohio State University, and Kiumars Kaveh, University of Pittsburgh.

Differential Equations and Applications, King-Yeung Lam and Yuan Lou, Ohio State University, and Qiliang Wu, Michigan State University. 
Function Spaces, Operator Theory, and Non-Linear Differential Operators, David Cruz-Uribe, University of Alabama, and Osvaldo Mendez, University of Texas.

Geometric Methods in Shape Analysis, Sebastian Kurtek and Tom Needham, Ohio State University.

Graph Theory, John Maharry, Ohio State University, Yue Zhao, University of Central Florida, and Xiangqian Zhou, Wright State University.

Homological Algebra, Ela Celikbas and Olgur Celikbas, West Virginia University.

Homotopy Theory, Ernest Fontes, John E. Harper, Crichton Ogle, and Gabriel Valenzuela, Ohio State University.

Lefschetz Properties, Juan Migliore, University of Notre Dame, and Uwe Nagel, University of Kentucky.

Mathematical Modeling of Neuronal Networks, Janet Best, Ohio State University, Alicia Prieto Langarica, Youngstown State University, and Pamela B. Pyzza, Ohio Wesleyan University.

Multiplicative Ideal Theory and Factorization (in honor of Tom Lucas retirement), Evan Houston, University of North Carolina, Charlotte, and Alan Loper, Ohio State University.

Noncommutative Algebra and Noncommutative Algebraic Geometry, Jason Gaddis, Miami University, and Robert Won, Wake Forest University.

Nonlinear Evolution Equations, John Holmes and Feride Tiglay, Ohio State University.

Nonlinear Waves and Patterns, Anna Ghazaryan, Miami University, Stephane Lafortune, College of Charleston, and Vahagn Manukian and Alin Pogan, Miami University.

Parameter Analysis and Estimation in Applied Dynamical Systems, Adriana Dawes, The Ohio State University, and Reginald L. McGee, Mathematical Biosciences Institute.

Probabilistic and Extremal Graph Theory, Louis DeBiasio and Tao Jiang, Miami University.

Probability in Convexity and Convexity in Probability, Elizabeth Meckes, Mark Meckes, and Elisabeth Werner, Case Western Reserve University.

Quantum Symmetries, David Penneys, The Ohio State University, and Julia Plavnik, Texas A \& M University.

Recent Advances in Approximation Theory and Operator Theory, Jan Lang and Paul Nevai, The Ohio State University.

Recent Advances in Finite Element Methods for Partial Differential Equations, Ching-shan Chou, Yukun Li, and Yulong Xing, The Ohio State University.

Recent Advances in Packing, Joseph W. Iverson, University of Maryland, John Jasper, South Dakota State University, and Dustin G. Mixon, The Ohio State University.

Recent Development of Nonlinear Geometric PDEs, Bo Guan, Ohio State University, Qun Li, Wright State University, Xiangwen Zhang, University of California, Irvine, and Fangyang Zheng, Ohio State University.

Several Complex Variables, Liwei Chen, Kenneth Koenig, and Liz Vivas, Ohio State University.
Stochastic Analysis in Infinite Dimensions, Parisa Fatheddin, Air Force Institute of Technology, and Arnab Ganguly, Louisiana State University.

Structure and Representation Theory of Finite Groups, Justin Lynd, University of Louisiana at Lafayette, and Hung Ngoc Nguyen, University of Akron.

Symmetry in Differential Geometry, Samuel Lin, Dartmouth College, Barry Minemyer, Bloomsburg University, and Ben Schmidt, Michigan State University.

The Mathematics of Phylogenetics, Colby Long, Mathematical Biosciences Institute.

Topology and Geometry in Data Analysis, Sanjeevi Krishnan and Facundo Memoli, Ohio State University.

\section{Nashville, Tennessee}

\section{Vanderbilt University}

April 14-15, 2018

Saturday - Sunday

\section{Meeting \# 1138}

Southeastern Section

Associate secretary: Brian D. Boe

Announcement issue of Notices: January 2018

Program first available on AMS website: February 22, 2018

Issue of Abstracts: Volume 39, Issue 2

\section{Deadlines}

For organizers: Expired

For abstracts: February 13, 2018

The scientific information listed below may be dated. For the latest information, see www.ams.org/amsmtgs/ sectional.htm 1 .

\section{Invited Addresses}

Andrea Bertozzi, University of California Los Angeles, Geometric graph-based methods for high dimensional data (Erdős Memorial Lecture).

Joseph (JM) Landsberg, Dept. of Mathematics, Texas A\&M Univeristy, On the geometry of matrix multiplication.

Jennifer Morse, University of Virginia, Combinatorics, Computing, and $k$-Schur functions.

Kirsten Wickelgren, Georgia Institute of Technology, An Arithmetic Count of the Lines on a Smooth Cubic Surface.

\section{Special Sessions}

If you are volunteering to speak in a Special Session, you should send your abstract as early as possible via the abstract submission form found at www . ams . org/cgi-bin/ abstracts/abstract.p1.

Advances in Operator Algebras (Code: SS 7A), Scott Atkinson, Dietmar Bisch, Vaughan Jones, and Jesse Peterson, Vanderbilt University. 
Algebraic Geometry, Representation Theory, and Applications (Code: SS 21A), Shrawan Kumar, University of North Carolina at Chapel Hill, J. M. Landsberg, Texas A\&M University, and Luke Oeding, Auburn University.

Boundaries and Non-positive Curvature in Group Theory (Code: SS 15A), Spencer Dowdall and Matthew Hallmark, Vanderbilt University, and Michael Hull, University of Florida.

Commutative Algebra (Code: SS 8A), Florian Enescu and Yongwei Yao, Georgia State University.

Evolution Equations and Applications (Code: SS 14A), Marcelo Disconzi, Chenyun Luo, Giusy Mazzone, and Gieri Simonett, Vanderbilt University.

Function Spaces and Operator Theory (Code: SS 9A), Cheng Chu and Dechao Zheng, Vanderbilt University.

Harmonic Analysis, Functional Analysis, and Their Applications (Code: SS 11A), Akram Aldroubi and Keaton Hamm, Vanderbilt University, Michael Northington, V, Georgia Institute of Technology, and Alex Powell, Vanderbilt University.

Hermitian Geometry (Code: SS 18A), Mehdi Lejmi, Bronx Community College of CUNY, and Rares Rasdeaconu and Ioana Suvaina, Vanderbilt University.

Interactions between Geometry, Group Theory and Dynamics (Code: SS 13A), Jayadev Athreya, University of Washington, and Caglar Uyanik and Grace Work, Vanderbilt University.

Macdonald Polynomials and Related Structures (Code: SS 23A), Jennifer Morse, University of Virginia, and Dan Orr and Mark Shimozono, Virginia Polytechnic Institute and State University.

Mathematical Chemistry (Code: SS 10A), Hua Wang, Georgia Southern University.

Matroids and Related Structures (Code: SS 5A), Carolyn Chun, United States Naval Academy, Deborah Chun and Tyler Moss, West Virginia University Institute of Technology, and Jakayla Robbins, Vanderbilt University.

Partial Differential Equations and New Perspective of Variational Methods (Code: SS 16A), Abbas Moameni, Carleton University, Futoshi Takahashi, Osaka City University, Michinori Ishiwata, Osaka University, and Craig Cowen, University of Manitoba.

Probabilistic Models in Mathematical Physics (Code: SS 6A), Robert Buckingham, University of Cincinnati, SeungYeop Lee, University of South Florida, and Karl Liechty, DePaul University.

Quantization for Probability Distributions and Dynamical Systems (Code: SS 1A), Mrinal Kanti Roychowdhury, University of Texas Rio Grande Valley.

Random Discrete Structures (Code: SS 22A), Lutz P Warnke, Georgia Institute of Technology, and Xavier Pérez-Giménez, University of Nebraska-Lincoln.

Recent Advances in Mathematical Biology (Code: SS 12A), Glenn Webb and Yixiang Wu, Vanderbilt University.

Recent Advances on Complex Bio-systems and Their Applications (Code: SS 17A), Pengcheng Xiao, University of Evansville.
Recent Progress and New Directions in Homotopy Theory (Code: SS 20A), Anna Marie Bohmann, Vanderbilt University, and Kirsten Wickelgren, Georgia Institute of Technology.

Selected Topics in Graph Theory (Code: SS 3A), Songling Shan, Vanderbilt University, and David Chris Stephens and Dong Ye, Middle Tennessee State University.

Structural Graph Theory (Code: SS 4A), Joshua Fallon, Louisiana State University, and Emily Marshall, Arcadia University.

Tensor Categories and Diagrammatic Methods (Code: SS 19A), Marcel Bischoff, Ohio University, and Henry Tucker, University of California San Diego.

\section{Portland, Oregon}

\section{Portland State University}

April 14-15, 2018

Saturday - Sunday

Meeting \# 1137

Western Section

Associate secretary: Michel L. Lapidus

Announcement issue of Notices: January 2018

Program first available on AMS website: February 15, 2018

Issue of Abstracts: Volume 39, Issue 2

\section{Deadlines}

For organizers: Expired

For abstracts: Expired

The scientific information listed below may be dated. For the latest information, see www.ams.org/amsmtgs/ sectional.htm 1 .

\section{Invited Addresses}

Sándor J Kovács, University of Washington, Moduli theory and singularities.

Elena Mantovan, California Institute of Technology, Title to be announced.

Dimitri Shlyakhtenko, University of California, Los Angeles, Title to be announced.

\section{Special Sessions}

If you are volunteering to speak in a Special Session, you should send your abstract as early as possible via the $a b$ stract submission form found at www. ams . org/cgi-bin/ abstracts/abstract.p1.

Algebraic Geometry and its Connections, Sándor Kovács, University of Washington, Seattle, and Karl Schwede, University of Utah, Salt Lake City.

Algebraic Topology, Angélica Osorno, Reed College, and Dev Sinha, University of Oregon.

Algebraic and Combinatorial Structures in Knot Theory, Allison Henrich, Seattle University, Inga Johnson, Wil- 
lamette University, and Sam Nelson, Claremont McKenna College.

Automorphisms of Riemann Surfaces and Related Topics, S. Allen Broughton, Rose-Hulman Institute of Technology, Mariela Carvacho, Universidad Tecnica Federico Santa Maria, Anthony Weaver, Bronx Community College, the City University of New York, and Aaron Wootton, University of Portland.

Biomathematics - Progress and Future Directions, Hannah Callender Highlander, University of Portland, Peter Hinow, University of Wisconsin - Milwaukee, and Deena Schmidt, University of Nevada, Reno.

Commutative Algebra, Adam Boocher, University of Utah, and Irena Swanson, Reed College.

Complex Analysis and Applications, Malik Younsi, University of Hawaii Manoa.

Differential Geometry, Christine Escher, Oregon State University, and Catherine Searle, Wichita State University.

Forest Modeling, Gatziolis Demetrios, Pacific Northwest Research Station, US Forest Service, and Nikolay Strigul, Washington State University, Vancouver.

General Relativity and Geometric Analysis, Paul T. Allen, Lewis \& Clark College, Jeffrey Jauregui, Union College, and Iva Stavrov Allen, Lewis \& Clark College.

Geometric Measure Theory and Partial Differential Equations, Mark Allen, Brigham Young University, and Spencer Becker-Kahn and Mariana Smit Vega Garcia, University of Washington.

Inverse Problems, Hanna Makaruk, Los Alamos National Laboratory (LANL), and Robert Owczarek, University of New Mexico, Albuquerque \& Los Alamos.

Mock Modular and Quantum Modular Forms, Holly Swisher, Oregon State University, and Stephanie Treneer, Western Washington University.

Modeling, Analysis, and Simulation of PDEs with Multiple Scales, Interfaces, and Coupled Phenomena, Yekaterina Epshteyn, University of Utah, and Malgorzata Peszynska, Oregon State University.

Moduli Spaces, Renzo Cavalieri, Colorado State University, and Damiano Fulghesu, Minnesota State University Moorhead.

Motivic homotopy theory, Daniel Dugger, University of Oregon, and Kyle Ormsby, Reed College.

Noncommutative Algebraic Geometry and Related Topics, Jesse Levitt, University of Southern California, Hans Nordstrom, University of Portland, and Xinting Wang, Temple University.

Nonsmooth Optimization and Applications(Dedicated to Prof. B. S. Mordukhovich on the occasion of his 70th birthday), Mau Nam Nguyen, Portland State University, Hung M. Phan, University of Massachusetts Lowell, and Shawn Xianfu Wang, University of British Columbia.

Numerical Methods for Partial Differential Equations, Brittany A. Erickson and Jeffrey S. Ovall, Portland State University.

Recent Advances in Actuarial Mathematics, Sooie-Hoe Loke, Central Washington University, and Enrique Thomann, Oregon State University.
Spectral Theory, Jake Fillman, Virginia Tech, and Milivoje Lukic, Rice University.

Teaching and Learning in Undergraduate Mathematics, Natalie LF Hobson, Sonoma State University, and Elise Lockwood, Oregon State University.

Wavelets, Frames, and Related Expansions, Marcin Bownik, University of Oregon, and Darrin Speegle, Saint Louis University.

\section{Boston, Massachusetts}

\section{Northeastern University}

April 21-22, 2018

Saturday - Sunday

\section{Meeting \#1 139}

Eastern Section

Associate secretary: Steven H. Weintraub

Announcement issue of Notices: January 2018

Program first available on AMS website: March 1, 2018

Issue of Abstracts: Volume 39, Issue 2

\section{Deadlines}

For organizers: Expired

For abstracts: February 20, 2018

The scientific information listed below may be dated. For the latest information, see www. ams.org/amsmtgs/ sectional.htm 1 .

\section{Invited Addresses}

Jian Ding, University of Pennsylvania, Random walk, random media and random geometry.

Edward Frenkel, University of California, Berkeley, Imagination and knowledge (Einstein Public Lecture in Mathematics).

Valentino Tosatti, Northwestern University, Metric limits of Calabi-Yau manifolds.

Maryna Viazovska, École Polytechnique Fédérale de Lausanne, Title to be announced.

\section{Special Sessions}

If you are volunteering to speak in a Special Session, you should send your abstract as early as possible via the abstract submission form found at www . ams . org/cgi-bin/ abstracts/abstract.p1.

Algebraic Number Theory (Code: SS 35A), Michael Bush, Washington and Lee University, Farshid Hajir, University of Massachusetts, and Christian Maire, Université Bourgogne Franche-Comté.

Algebraic Statistics (Code: SS 33A), Kaie Kubjas and Elina Robeva, Massachusetts Institute of Technology. 
Algebraic, Geometric, and Topological Methods in Combinatorics (Code: SS 21A), Florian Frick, Cornell University, and Pablo Soberón, Northeastern University.

Algorithmic Group Theory and Applications (Code: SS 26A), Delaram Kahrobaei, City University of New York, and Antonio Tortora, University of Salerno.

Analysis and Geometry in Non-smooth Spaces (Code: SS 5A), Nageswari Shanmugalingam and Gareth Speight, University of Cincinnati.

Arithmetic Dynamics (Code: SS 1A), Jacqueline M. Anderson, Bridgewater State University, Robert Benedetto, Amherst College, and Joseph H. Silverman, Brown University.

Arrangements of Hypersurfaces (Code: SS 2A), Graham Denham, University of Western Ontario, and Alexander I. Suciu, Northeastern University.

Combinatorial Aspects of Nilpotent Orbits (Code: SS 15A), Anthony Iarrobino, Northeastern University, Leila Khatami, Union College, and Juliana Tymoczko, Smith College.

Combinatorial Representation Theory (Code: SS 41A), Laura Colmenarejo, York University, Ricky Liu, North Carolina State University, and Rosa Orellana, Dartmouth College.

Connections Between Trisections of 4-manifolds and Low-dimensional Topology (Code: SS 32A), Jeffrey Meier, University of Georgia, and Juanita Pinzon-Caicedó, North Carolina State University.

Discretization in Geometry and Dynamics (Code: SS 36A), Richard Kenyon, Wai Yeung Lam, and Richard Schwartz, Brown University.

Dynamical systems, Geometric Structures and Special Functions (Code: SS 23A), Alessandro Arsie, University of Toledo, and Oksana Bihun, University of Colorado, Colorado Springs.

Effective Behavior in Random Environments (Code: SS 25A), Jessica Lin, McGill University, and Charles Smart, University of Chicago.

Ergodic Theory and Dynamics in Combinatorial Number Theory (Code: SS 7A), Stanley Eigen and Daniel Glasscock, Northeastern University, and Vidhu Prasad, University of Massachusetts, Lowell.

Extremal Graph Theory and Quantum Walks on Graphs (Code: SS 13A), Sebastian Cioabă, University of Delaware, Mark Kempton, Harvard University, Gabor Lippner, Northeastern University, and Michael Tait, Carnegie Mellon University.

Facets of Symplectic Geometry and Topology (Code: SS 3A), Tara Holm, Cornell University, Jo Nelson, Columbia University, and Jonathan Weitsman, Northeastern University.

Geometries Defined by Differential Forms (Code: SS 34A), Mahir Bilen Can, Tulane University, Sergey Grigorian, University of Texas Rio Grande Valley, and Sema Salur, University of Rochester.

Geometry and Analysis of Fluid Equations (Code: SS 28A), Robert McOwen and Peter Topalov, Northeastern University.
Geometry of Moduli Spaces (Code: SS 10A), Ana-Marie Castravet and Emanuele Macrí, Northeastern University, Benjamin Schmidt, University of Texas, and Xiaolei Zhao, Northeastern University.

Global Dynamics of Real Discrete Dynamical Systems (Code: SS 30A), M. R. S. Kulenović and O. Merino, University of Rhode Island.

Harmonic Analysis and Partial Differential Equations (Code: SS 29A), Donatella Danielli, Purdue University, and Irina Mitrea, Temple University.

Homological Commutative Algebra (Code: SS 11A), Sean Sather-Wagstaff, Clemson University, and Oana Veliche, Northeastern University.

Hopf Algebras, Tensor Categories, and Homological Algebra (Code: SS 8A), Cris Negron, Massachusetts Institute of Technology, Julia Plavnik, Texas A\&M, and Sarah Witherspoon, Texas A\&M University.

Mathematical Perspectives in Quantum Information Theory (Code: SS 24A), Aram Harrow, Massachusetts Institute of Technology, and Christopher King, Northeastern University.

Mathematical Problems of Relativistic Physics: Classical and Quantum (Code: SS 37A), Michael Kiessling and A. Shadi Tahvildar-Zadeh, Rutgers University.

Modeling of Biological Processes (Code: SS 38A), Simone Cassani and Sarah Olson, Worcester Polytechnic Institute.

New Developments in Inverse Problems and Imaging (Code: SS 9A), Ru-Yu Lai, University of Minnesota, and Ting Zhou, Northeastern University.

Noncommutative Algebra and Representation Theory (Code: SS 22A), Van C. Nguyen, Hood College, and Alex Martsinkovsky and Gordana Todorov, Northeastern University.

Nonlinear Reaction-Diffusion Equations and Their Applications (Code: SS 31A), Nsoki Mavinga and Quinn Morris, Swarthmore College.

Nonlinear and Stochastic Partial Differential Equations and Applications (Code: SS 19A), Nathan Glatt-Holtz and Vincent Martinez, Tulane University, and Cecilia Mondaini, Texas A\&M University.

Numerical Methods and Applications (Code: SS 16A), Vera Babenko, Ithaca College.

Optimization Under Uncertainty (Code: SS 40A), Yingdong Lu and Mark S. Squillante, IBM Research.

Polytopes and Discrete Geometry (Code: SS 6A), Gabriel Cunningham, University of Massachusetts, Boston, Mark Mixer, Wentworth Institute of Technology, and Egon Schulte, Northeastern University.

Regularity of PDEs on Rough Domains (Code: SS 14A), Murat Akman, University of Connecticut, and Max Engelstein, Massachusetts Institute of Technology.

Relations Between the History and Pedagogy of Mathematics (Code: SS 20A), Amy Ackerberg-Hastings, and David L. Roberts, Prince George's Community College.

Singularities of Spaces and Maps (Code: SS 4A), Terence Gaffney and David Massey, Northeastern University. 


\section{MEETINGS \& CONFERENCES}

The Analysis of Dispersive Equations (Code: SS 39A), Marius Beceanu, University at Albany, and Andrew Lawrie, Massachusetts Institute of Technology.

The Gaussian Free Field and Random Geometry (Code: SS 12A), Jian Ding, University of Pennsylvania, and Vadim Gorin, Massachusetts Institute of Technology.

Topics in Qualitative Properties of Partial Differential Equations (Code: SS 27A), Changfeng Gui, University of Texas at San Antonio, Changyou Wang, Purdue University, and Jiuyi Zhu, Louisiana State University.

Topics in Toric Geometry (Code: SS 17A), Ivan Martino, Northeastern University, and Emanuele Ventura, Texas A\&M University.

Topology of Biopolymers (Code: SS 18A), Erica Flapan, Pomona College, and Helen Wong, Carleton College.

\section{Shanghai, People's Republic of China}

\section{Fudan University}

June 11-14, 2018

Monday - Thursday

\section{Meeting \# 1140}

Associate secretary: Steven H. Weintraub

Announcement issue of Notices: April 2018

Program first available on AMS website: Not applicable

Program issue of electronic Notices: Not applicable

Issue of Abstracts: Not applicable

\section{Deadlines}

For organizers: To be announced

For abstracts: To be announced

The scientific information listed below may be dated. For the latest information, see www.ams.org/amsmtgs/ internmtgs.htm 1 .

\section{Invited Addresses}

Yu-Hong Dai, Academy of Mathematics and System Sciences, Title to be announced.

Kenneth A. Ribet, University of California, Berkeley, Title to be announced.

Richard M. Schoen, University of California, Irvine, Title to be announced.

Sijue Wu, University of Michigan, Title to be announced. Chenyang Xu, Peking University, Title to be announced. Jiangong You, Nankai University, Title to be announced.

\section{Newark, Delaware}

\section{University of Delaware}

September 29-30, 2018

Saturday - Sunday

\section{Meeting \# 1141}

Eastern Section

Associate secretary: Steven H. Weintraub

Announcement issue of Notices: June 2018

Program first available on AMS website: August 9, 2018

Issue of Abstracts: Volume 39, Issue 3

\section{Deadlines}

For organizers: February 28, 2018

For abstracts: July 31, 2018

The scientific information listed below may be dated. For the latest information, see www. ams.org/amsmtgs/ sectional.htm 1 .

\section{Invited Addresses}

Leslie Greengard, New York University, Title to be announced.

Elisenda Grigsby, Boston College, Title to be announced.

Davesh Maulik, Massachusetts Institute of Technology, Title to be announced.

\section{Special Sessions}

If you are volunteering to speak in a Special Session, you should send your abstract as early as possible via the $a b$ stract submission form found at www. ams . org/cgi-bin/ abstracts/abstract.p1.

Applied Algebraic Topology (Code: SS 2A), Chad Giusti, University of Delaware, and Gregory Henselman, Princeton University.

Convex Geometry and Functional Inequalities (Code: SS 3A), Mokshay Madiman, University of Delaware, Elisabeth Werner, Case Western Reserve University, and Artem Zvavitch, Kent State University.

Fixed Point Theory with Application and Computation (Code: SS 6A), Clement Boateng Ampadu, Boston, MA.

Interplay between Analysis and Combinatorics (Code: SS 5A), Mahya Ghandehari and Dominique Guillot, University of Delaware.

Operator and Function Theory (Code: SS 4A), Kelly Bickel, Bucknell University, Michael Hartz, Washington University, St. Louis, Constanze Liaw, University of Delaware, and Alan Sola, Stockholm University.

Recent Advances in Nonlinear Schrödinger Equations (Code: SS 1A), Alexander Pankov, Morgan State University, Junping Shi, College of William and Mary, and Jun Wang, Jiangsu University. 


\section{Ann Arbor, Michigan}

University of Michigan, Ann Arbor

October 20-21, 2018

Saturday - Sunday

\section{Meeting \# 1143}

Central Section

Associate secretary: Georgia Benkart

Announcement issue of Notices: July 2018

Program first available on AMS website: August 30, 2018

Issue of Abstracts: Volume 39, Issue 4

\section{Deadlines}

For organizers: March 20, 2018

For abstracts: August 21, 2018

The scientific information listed below may be dated. For the latest information, see www.ams.org/amsmtgs/ sectiona1. htm?.

\section{Invited Addresses}

Elena Fuchs, University of Illinois Urbana-Champaign, Title to be announced.

Andrew Putman, University of Notre Dame, Title to be announced.

Charles Smart, University of Chicago, Title to be announced.

\section{Special Sessions}

If you are volunteering to speak in a Special Session, you should send your abstract as early as possible via the abstract submission form found at www . ams . org/cgi -bin/ abstracts/abstract.p1.

Cluster Algebra, Poisson Geometry, and Related Topics (Code: SS 9A), Eric Bucher, Michigan State University, and Maitreyee Kulkarni and Bach Nguyen, Louisiana State University.

From Hyperelliptic to Superelliptic Curves (Code: SS 6A), Tony Shaska, Oakland University, Nicola Tarasca, Rutgers University, and Yuri Zarhin, Pennsylvania State University. Geometry of Submanifolds, in Honor of Bang-Yen Chens 75th Birthday (Code: SS 1A), Alfonso Carriazo, University of Sevilla, Ivko Dimitric, Penn State Fayette, Yun Myung Oh, Andrews University, Bogdan D. Suceava, California State University, Fullerton, Joeri Van der Veken, University of Leuven, and Luc Vrancken, Universite de Valenciennes.

Interactions between Algebra, Machine Learning and Data Privacy (Code: SS 3A), Jonathan Gryak, University of Michigan, Kelsey Horan, CUNY Graduate Center, Delaram Kahrobaei, CUNY Graduate Center and New York University, Kayvan Najarian and Reza Soroushmehr, University of Michigan, and Alexander Wood, CUNY Graduate Center.
Large Cardinals and Combinatorial Set Theory (Code: SS 10A), Andres E. Caicedo, Mathematical Reviews, and Paul B. Larson, Miami University.

Probabilistic Methods in Combinatorics (Code: SS 7A), Patrick Bennett and Andrzej Dudek, Western Michigan University, and David Galvin, University of Notre Dame.

Random Matrix Theory Beyond Wigner and Wishart (Code: SS 2A), Elizabeth Meckes and Mark Meckes, Case Western Reserve University, and Mark Rudelson, University of Michigan.

Representations of Reductive Groups over Local Fields and Related Topics (Code: SS 8A), Anne-Marie Aubert, Institut Mathématiques de Jussieu, Paris Rive Gauche, Jessica Fintzen, IAS, University of Michigan, University of Cambridge, and Camelia Karimianpour, University of Michigan.

Self-similarity and Long-range Dependence in Stochastic Processes (Code: SS 4A), Takashi Owada, Purdue University, Yi Shen, University of Waterloo, and Yizao Wang, University of Cincinnati.

Structured Homotopy Theory (Code: SS 5A), Thomas Fiore, University of Michigan, Dearborn, Po Hu and Dan Isaksen, Wayne State University, and Igor Kriz, University of Michigan.

\section{San Francisco, California \\ San Francisco State University}

October 27-28, 2018

Saturday - Sunday

Meeting \# 1144

Western Section

Associate secretary: Michel L. Lapidus

Announcement issue of Notices: July 2018

Program first available on AMS website: September 6, 2018 Issue of Abstracts: Volume 39, Issue 4

\section{Deadlines}

For organizers: March 27, 2018

For abstracts: August 28, 2018

The scientific information listed below may be dated. For the latest information, see www.ams.org/amsmtgs/ sectional.htm 1 .

\section{Invited Addresses}

Srikanth B. Iyengar, University of Utah, Title to be announced.

Sarah Witherspoon, Texas A\&M University, Title to be announced.

Abdul-Aziz Yakubu, Howard University, Title to be announced. 


\section{Special Sessions}

If you are volunteering to speak in a Special Session, you should send your abstract as early as possible via the abstract submission form found at www. ams . org/cgi-bin/ abstracts/abstract.p1.

Coupling in Probability and Related Fields (Code: SS 3A), Sayan Banerjee, University of North Carolina, Chapel Hill, and Terry Soo, University of Kansas.

Geometric and Analytic Inequalities and their Applications (Code: SS 4A), Nicholas Brubaker, Isabel M. Serrano, and Bogdan D. Suceavă, California State University, Fullerton.

Homological Aspects in Commutative Algebra and Representation Theory (Code: SS 5A), Srikanth B. Iyengar, University of Utah, and Julia Pevtsova, University of Washington.

Homological Aspects of Noncommutative Algebra and Geometry (Code: SS 2A), Dan Rogalski, University of California San Diego, Sarah Witherspoon, Texas A\&M University, and James Zhang, University of Washington, Seattle.

Mathematical Biology with a focus on Modeling, Analysis, and Simulation (Code: SS 1A), Jim Cushing, The University of Arizona, Saber Elaydi, Trinity University, Suzanne Sindi, University of California, Merced, and Abdul-Aziz Yakubu, Howard University.

\section{Fayetteville, Arkansas}

\section{University of Arkansas}

\section{November 3-4, 2018}

Saturday - Sunday

\section{Meeting \# 1142}

Southeastern Section

Associate secretary: Brian D. Boe

Announcement issue of Notices: July 2018

Program first available on AMS website: August 16, 2018 Issue of Abstracts: Volume 39, Issue 3

\section{Deadlines}

For organizers: April 3, 2018

For abstracts: September 4, 2018

The scientific information listed below may be dated. For the latest information, see www.ams.org/amsmtgs/ sectional.html.

\section{Invited Addresses}

Mihalis Dafermos, Princeton University, Title to be announced.
Jonathan Hauenstein, University of Notre Dame, Title to be announced.

Kathryn Mann, University of California Berkeley, Title to be announced.

\section{Baltimore, Maryland}

\section{Baltimore Convention Center, Hilton} Baltimore, and Baltimore Marriott Inner Harbor Hotel

\author{
January 16-19, 2019 \\ Wednesday - Saturday
}

\section{Meeting \# 1145}

Joint Mathematics Meetings, including the 125th Annual Meeting of the AMS, 102nd Annual Meeting of the Mathematical Association of America (MAA), annual meetings of the Association for Women in Mathematics (AWM)and the National Association of Mathematicians (NAM), and the winter meeting of the Association of Symbolic Logic (ASL), with sessions contributed by the Society for Industrial and Applied Mathematics (SIAM).

Associate secretary: Steven H. Weintraub

Announcement issue of Notices: October 2018

Program first available on AMS website: To be announced Issue of Abstracts: To be announced

\section{Deadlines}

For organizers: April 2, 2018

For abstracts: To be announced

\section{Call for Proposals}

Steven H. Weintraub, Associate Secretary responsible for the AMS program at the 2019

Joint Mathematics Meetings (to be held from Wednesday, January 16 through Saturday, January 19, 2019, in Baltimore, MD) solicits proposals for Special Sessions for this meeting. Each proposal must include:

1. the name, affiliation, and email address of each organizer, with one organizer designated as the contact person for all communication about the session;

2. the title and a brief (two or three paragraph) description of the topic of the proposed special session;

3. a sample list of speakers whom the organizers plan to invite. (It is not necessary to have received confirmed commitments from these potential speakers.)

Organizers are encouraged to read the AMS Manual for Special Session Organizers

at: www. ams.org/meetings/meet-specialsessionmanual in its entirety.

Proposals for AMS Special Sessions should be sent by email to Prof. Weintraub (shw2@1ehigh.edu) and must be received by the deadline for organizers, April 1, 2018. Late proposals will not be considered. No decisions will be made on Special Session proposals until after the submission deadline has passed. 
Special Sessions will in general be allotted between 5 and 10 hours in which to schedule speakers. To enable maximum movement of participants between sessions, organizers must schedule each speaker for either a 20-minute talk, 5-minute discussion, and 5-minute break; or a 45-minute talk, 10-minute discussion, and 5-minute break. Any combination of 20-minute and 45-minute talks is permitted, but all talks should begin and end at the scheduled time.

The number of Special Sessions on the AMS program at the Joint Mathematics Meetings is limited, and not all proposals can be accepted. Please be sure to submit as detailed a proposal as possible for review by the Program Committee. We aim to notify organizers whether their proposal has been accepted by May 1, 2018.

\section{Auburn, Alabama}

\section{Auburn University}

\section{March 15-17,2019 \\ Friday - Sunday}

\section{Meeting \# 1146}

Southeastern Section

Associate secretary: Brian D. Boe

Announcement issue of Notices: To be announced

Program first available on AMS website: To be announced Issue of Abstracts: To be announced

\section{Deadlines}

For organizers: To be announced

For abstracts: To be announced

\section{Honolulu, Hawaii}

\section{University of Hawaii at Manoa}

\section{March 22-24, 2019}

Friday - Sunday

\section{Meeting \# 1147}

Central Section and Western

Associate secretaries: Georgia Benkart and Michel L. Lapidus

Announcement issue of Notices: To be announced

Program first available on AMS website: To be announced Issue of Abstracts: To be announced

\section{Deadlines}

For organizers: May 15, 2018

For abstracts: January 22, 2019

The scientific information listed below may be dated. For the latest information, see www. ams.org/amsmtgs/ sectiona $1 . \mathrm{htm} 7$.

\section{Invited Addresses}

Barry Mazur, Harvard University, Title to be announced (Einstein Public Lecture in Mathematics).

Aaron Naber, Northwestern University, Title to be announced.

Deanna Needell, University of California, Los Angeles, Title to be announced.

Katherine Stange, University of Colorado, Boulder, Title to be announced.

Andrew Suk, University of Illinois at Chicago, Title to be announced.

\section{Hartford, Connecticut}

\section{University of Connecticut Hartford (Hart- ford Regional Campus)}

April 13-14, 2019

Saturday - Sunday

\section{Meeting \# 1148}

Eastern Section

Associate secretary: Steven H. Weintraub

Announcement issue of Notices: To be announced Program first available on AMS website: To be announced Issue of Abstracts: To be announced

\section{Deadlines}

For organizers: September 13, 2018

For abstracts: To be announced

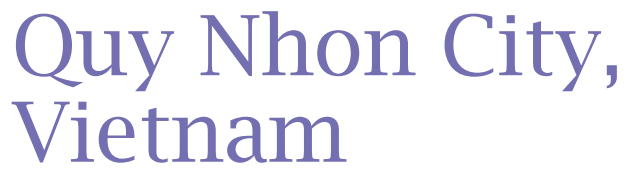

Quy Nhon University

June 10-13, 2019

Monday - Thursday

Meeting \# 1149

Associate secretary: Brian D. Boe

Announcement issue of Notices: To be announced

Program first available on AMS website: To be announced Issue of Abstracts: To be announced

\section{Deadlines}

For organizers: To be announced

For abstracts: To be announced 


\section{MEETINGS \& CONFERENCES}

\section{Binghamton, New York}

\section{Binghamton University}

October 12-13, 2019

Saturday - Sunday

Eastern Section

Associate secretary: Steven H. Weintraub

Announcement issue of Notices: To be announced

Program first available on AMS website: To be announced

Issue of Abstracts: To be announced

\section{Deadlines}

For organizers: March 12, 2019

For abstracts: To be announced

\section{Gainesville, Florida}

\section{University of Florida}

\section{November 2-3, 2019}

Saturday - Sunday

Southeastern Section

Associate secretary: Brian D. Boe

Announcement issue of Notices: To be announced

Program first available on AMS website: To be announced Issue of Abstracts: To be announced

\section{Deadlines}

For organizers: To be announced

For abstracts: To be announced

\section{Denver, Colorado}

\section{Colorado Convention Center}

January 15-18, 2020

Wednesday - Saturday

Joint Mathematics Meetings, including the 126th Annual Meeting of the AMS, 103rd Annual Meeting of the Mathematical Association of America (MAA), annual meetings of the Association for Women in Mathematics (AWM) and the National Association of Mathematicians (NAM), and the winter meeting of the Association of Symbolic Logic (ASL), with sessions contributed by the Society for Industrial and Applied Mathematics (SIAM)

Associate secretary: Michel L. Lapidus

Announcement issue of Notices: October 2019

Program first available on AMS website: November 1, 2019 Issue of Abstracts: To be announced

\section{Deadlines}

For organizers: April 1, 2019

For abstracts: To be announced

\section{Washington, District of Columbia}

\section{Walter E. Washington Convention Center}

\section{January 6-9, 2021}

Wednesday - Saturday Joint Mathematics Meetings, including the 127th Annual Meeting of the AMS, 104th Annual Meeting of the Mathematical Association of America (MAA), annual meetings of the Association for Women in Mathematics (AWM) and the National Association of Mathematicians (NAM), and the winter meeting of the Association of Symbolic Logic (ASL), with sessions contributed by the Society for Industrial and Applied Mathematics (SIAM).

Associate secretary: Brian D. Boe

Announcement issue of Notices: October 2020

Program first available on AMS website: November 1, 2020 Issue of Abstracts: To be announced

\section{Deadlines}

For organizers: April 1, 2020

For abstracts: To be announced 


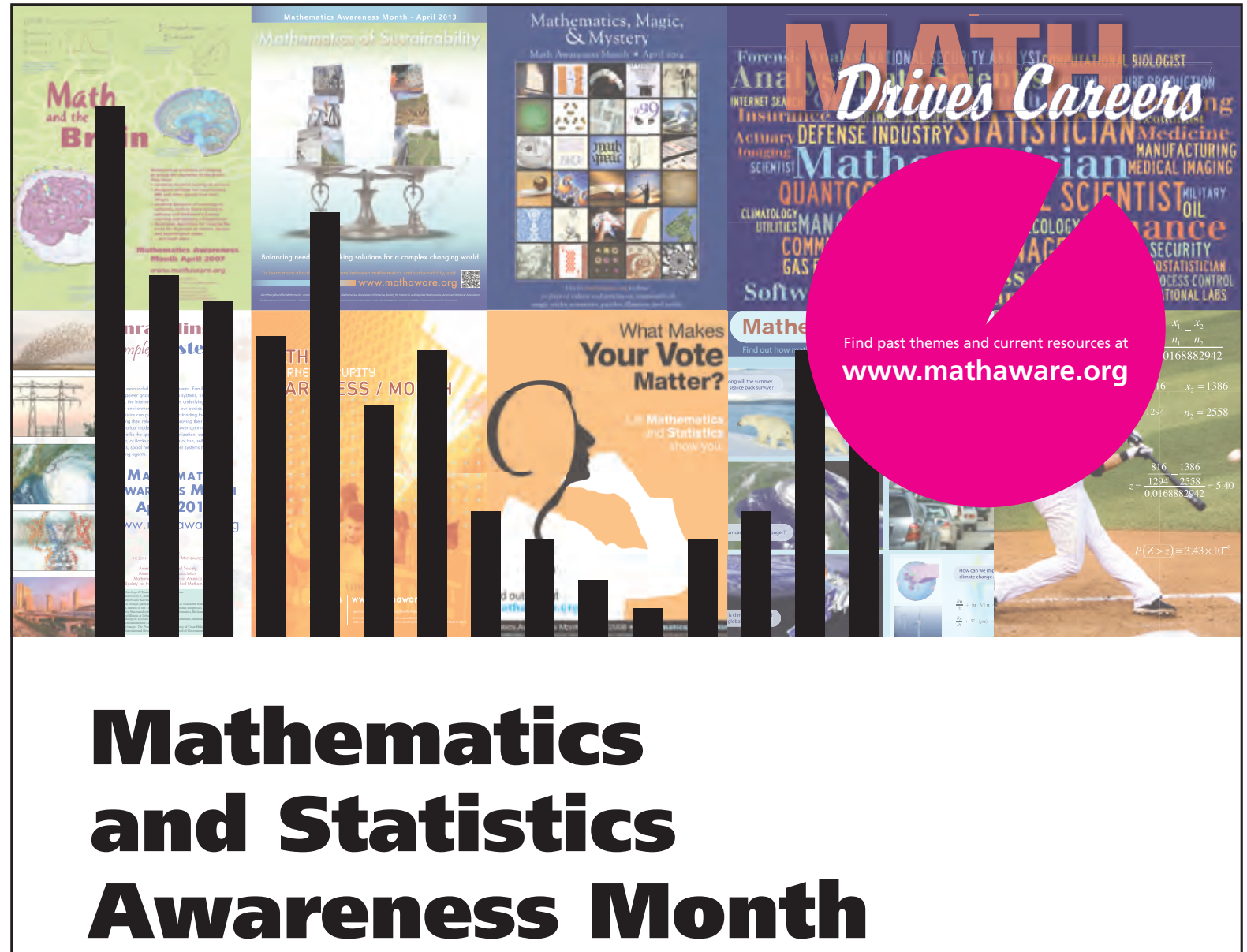

April marks a time to increase the understanding and appreciation of mathematics and statistics. Why? Both subjects play a significant role in addressing many real-world problems-climate change, disease, sustainability, the data deluge, internet security, and much more. Research in these and other areas is ongoing, revealing new results and applications every day in fields such as medicine, manufacturing, energy, biotechnology, and business. Mathematics and statistics are important drivers of innovation in our technological world, in which new systems and methodologies continue to become more complex.

Organize and host activities in April for Mathematics and Statistics Awareness Month! Past activities have included workshops, competitions, festivals, lectures, symposia, department open houses, math art exhibits, and math poetry readings. Share your activities on social media.

MathAware

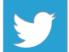

@MathAware 


\section{IN THE NEXT ISSUE OF NOTICES}

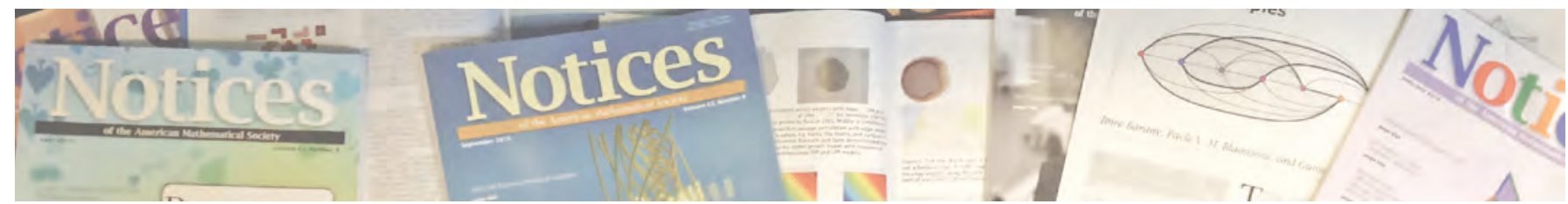

\section{APRIL 2018}

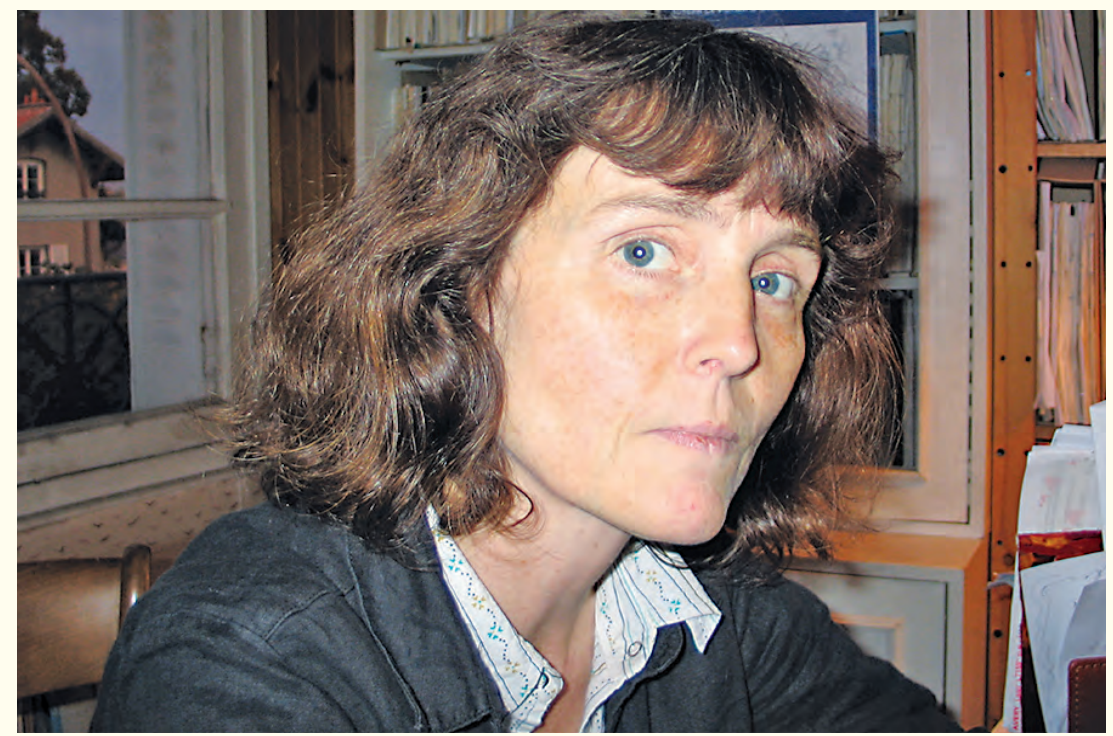

\section{Ad Honorem Claire Voisin}

We honor Voisin, world leader in algebraic geometry and recent winner of the Shaw Prize and the CNRS Gold Medal.

\section{Mathematics and Statistics} Awareness Month:

Scoring with New Thinking A Math Moment with Andy Andres

Mathematically Modelling Baseball by Bruce Bukiet

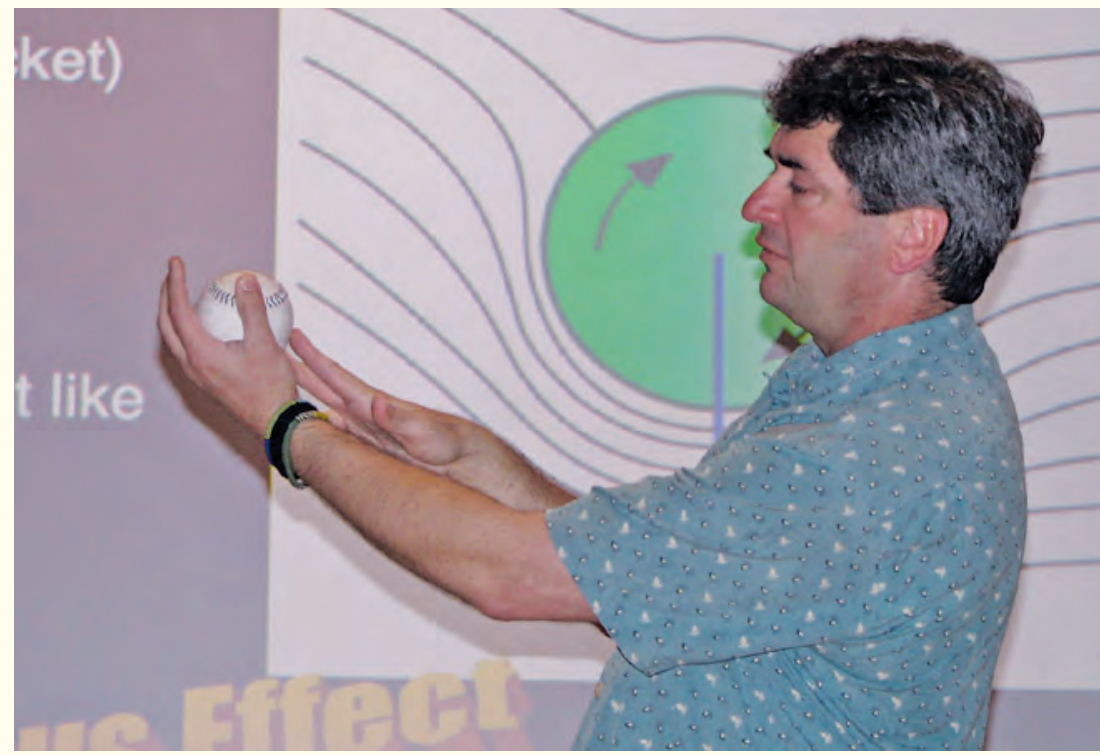




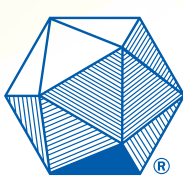

\section{of the}

An Imprint

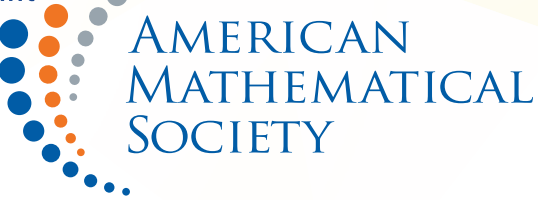

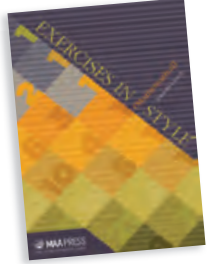

O AVAILABLE IN EBOOK FORMAT

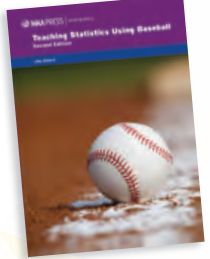

- TEXTBOOK

E. AVAILABLE IN EBOOK FORMAT

\section{Exercises in (Mathematical) Style}

Stories of Binomial Coefficients

John McCleary

By exploring the rich properties of binomial coefficients and investigating the world of that familiar set of numbers, this book celebrates the joy of mathematics and the joy of writing mathematics.

Anneli Lax New Mathematical Library, Volume 48; 2017; 275 pages; Softcover; ISBN: 978-0-88385-652-9; List US $\$ 48$; Individual member US $\$ 36$; Order code NML/48

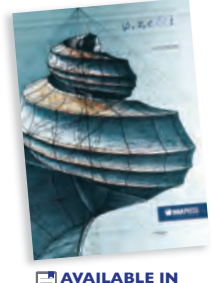
FBVALABLE IN

\section{Teaching Statistics \\ Using Baseball}

Second Edition

Jim Albert

This book illustrates basic methods of data analysis and probability models by means of baseball statistics collected on players and teams.

AMS/MAA Textbooks, Volume 34; 2017; 243 pages; Softcover; ISBN: 978-1-93951-216-1; List US\$55; Individual member US\$41.25; Order code TEXT/34

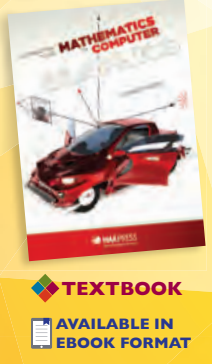

\section{Introduction to the Mathematics of Computer Graphics}

Nathan Carter, Bentley University, Waltham, MA

In this text, designed to accompany a firstyear seminar in the mathematics of computer graphics, readers learn the mathematics behind the computational aspects of space, shape, transformation, color, rendering, animation, and modeling.

Classroom Resource Materials, Volume 51; 2016; 462 pages; ISBN: 978-1-61444-122-9; List US\$50; Individual member US\$37.50; Order code CLRM/51.E

\section{$\Phi, \Pi, e$, and $i$}

\section{David Perkins}

Certain constants occupy precise balancing points in the cosmos of number, like habitable planets sprinkled throughout our galaxy at just the right distances from their suns. This book introduces and connects four of these constants (Phi, Pi, e and i) at a level appropriate for an audience armed only with the tools of elementary calculus.

Spectrum, Volume 86; 2017; 176 pages; Softcover; ISBN: 978-0-88385-589-8; List US\$50; Individual member US $\$ 37.50$; Order code SPEC/86

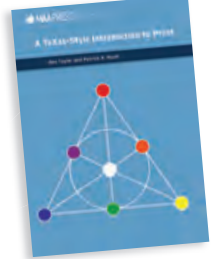

TеХтвоOK

FAVAILABLE IN EBOOK FORMAT

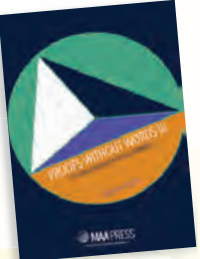
AVAILABLE IN
EBOOK FORMAT EBOOK FORMAT

\section{al}


American Mathematical Society

Distribution Center

35 Monticello Place,

Pawtucket, RI 02861 USA
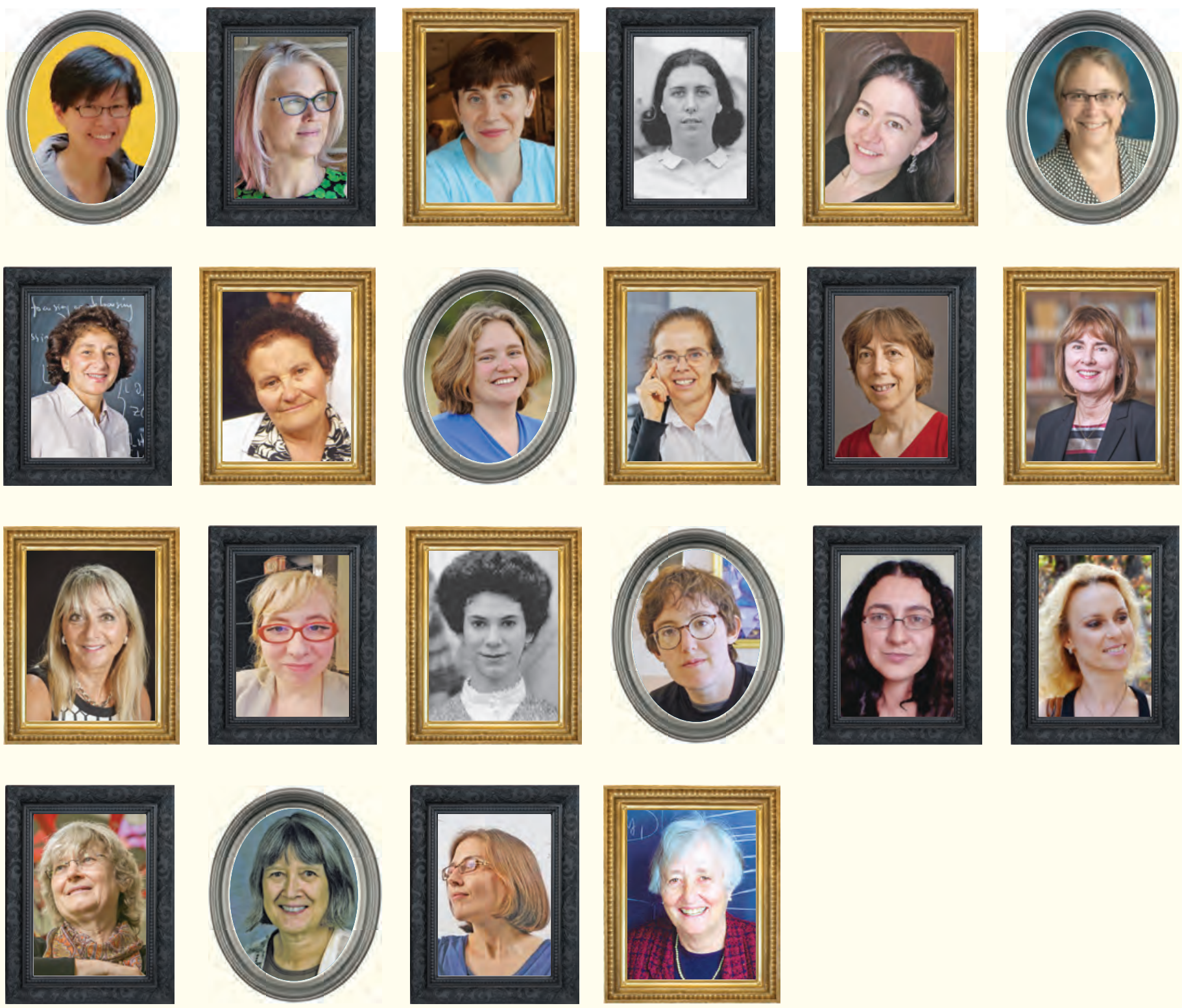